\title{
Biologically Plausible Methods for Robot Visual Homing
}

\author{
By \\ Andrew Vardy \\ A thesis submitted to \\ the Faculty of Graduate Studies and Research \\ in partial fulfilment of \\ the requirements for the degree of \\ Doctor of Philosophy
}

Ottawa-Carleton Institute for Computer Science
School of Computer Science
Carleton University
Ottawa, Ontario

March 2005

(C) Copyright

2005, Andrew Vardy 
Library and

Archives Canada

Published Heritage

Branch

395 Wellington Street Ottawa ONK1A ON4

Canada
Bibliothèque et

Archives Canada

$0-494-06748-9$

Direction du

Patrimoine de l'édition

395 , rue Wellington

OHawa ON K1A ON4

Canada
NOTICE:

The author has granted a nonexclusive license allowing Library and Archives Canada to reproduce, publish, archive, preserve, conserve, communicate to the public by telecommunication or on the Internet, loan, distribute and sell theses worldwide, for commercial or noncommercial purposes, in microform, paper, electronic and/or any other formats.

The author retains copyright ownership and moral rights in this thesis. Neither the thesis nor substantial extracts from it may be printed or otherwise reproduced without the author's permission.
AVIS:

L'auteur a accordé une licence non exclusive permettant à la Bibliothèque et Archives Canada de reproduire, publier, archiver, sauvegarder, conserver, transmettre au public par télécommunication ou par l'Internet, prêter, distribuer et vendre des thèses partout dans le monde, à des fins commerciales ou autres, sur support microforme, papier, électronique etou autres formats.

L'auteur conserve la propriété du droit d'auteur et des droits moraux qui protège cette thèse. Ni la thèse ni des extraits substantiels de celle-ci ne doivent être imprimés ou autrement reproduits sans son autorisation.
In compliance with the Canadian Privacy Act some supporting forms may have been removed from this thesis.

While these forms may be included in the document page count, their removal does not represent any loss of content from the thesis.
Conformément à la loi canadienne sur la protection de la vie privée, quelques formulaires secondaires ont été enlevés de cette thèse.

Bien que ces formulaires aient inclus dans la pagination, il n'y aura aucun contenu manquant.

\section{Canada}




\section{Abstract}

This thesis concerns visual homing in robots and proposes solutions judged by both performance and biological plausibility: Visual homing is the ability to return to a goal from a nearby location using vision. Biological plausibility is defined as the likelihood that a proposed algorithm mirrors the internal mechanism of an animal solving the same problem. The high effectiveness of insect homing motivates interest in biological plausibility: The approach to biological plausibility is through retinotopic processing-a characteristic of natural visual systems.

Experiments are conducted on a database of panoramic images collected from a variety of indoor environments. A review of existing methods reveals Franz et al.'s [26] warping method as the de facto standard for robot visual homing. The warping method is compared with two other methods which adhere to the constraints of retinotopic processing. Its performance is found to be superior and is therefore used for subsequent performance comparisons.

The first new homing method proposed is an extension of Möller et al. $\$$ s [ 76$]$ neural snapshot model. It is found to be applicable to real-world images, but is not competitive with the warping method. Two other new methods are then proposed based on a novel scale invariant image descriptor. One of these is found to perform nearly as well as the warping method. Further, its scale invariance property is found not to be crucial to its success. Thus paving the way for simpler methods.

The first such simpler method, based on block matching, is found to outpperform the warping method. Analysis reveals that this method succeeds by pairing lowfrequency image components and that increasing the number of correspondences is beneficial. Two even simpler variants based on matching pixel intensity and the gradient of intensity were also found to perform quite well. 
The success of these variants prompts an investigation into differential-based techniques. Two methods are proposed and both found to perform competitively: These methods are found to be successful because of the presence of regions of small feature shifts in the image near the foci of expansion and contraction. The differential methods satisfy both the performance and biological plausibility criteria. 


\section{Acknowledgments}

Without inspiration and encouragement from a number of people, this thesis could never have begun. Without the help and support of others, it might have taken 40 years to complete, instead of just four.

I am grateful to Dr. Thomas Collett who inspired my interest in this topic through a course called "Animal and Machine Intelligence" at the University of Sussex. It is quite fitting that he will be serving as my external examiner, and for this I am also grateful. I am thankful also for inspiration from some curious hovering flies discovered one summer afternoon at my parent's home in Chamberlains, Newfoundland.

This work began in earnest at Carleton University in Ottawa. Dr. Franz Oppacher has been $m y$ academic Supervisor throughout my studies at Carleton and has allowed me great freedom to pursue an independent course of research. He has never failed in paving the way for me and I am most grateful for his support. Meetings of the ALEC group at Carleton were also a great source for icleas. I am thankful in particular to $\mathrm{Dr}$. Hassan Masum, Steffen Christensen, Lee Graham, Dr. Alex Tulai. Michael Iles, Ben Houston, Dr. George Carmody; and Dr. James Cheethann. Other faculty members at the School of Computer Science at Carleton have been generous with their time and advice in a variety of matters. I would like to thank Dr. Jason Morrison, Dr. Irwin Reichstein, and Dr. Mark Lanthier. The School of Computer Science has an exceilent administrative staff (both past and present). I am most grateful to Linda Pfeiffer, Maureen Sherman, Nicole Enouy, Sandy Dare, and Jane MacArthur.

I fear that I owe an unpayable debt of gratitude to Dr. Ralf Möller. His work and that of his colleagues in Zurich and Munich provided crucial inspiration. Beginning with an exchange of e-mail, Dr. Möller provided invaluable assistance in replicating some previous results. Over the following months he continued to provide enormously helpful advice and guidance. In September of 2003 we met in his lab in Bielefeld. 
He later commented that it was nice for us to meet and for him to finally do some science after several months spent setting up his new lab. This small comment had a profound effect on me when I realized that my work could actually be considered part of science, and more than just games of simulation and modelling. I came to Bielefeld for a crucial two-month visit in February and March of 2004. This visit not only afforded me access to the exact robotic equipment necessary for me research, but gave Dr. Möller and I the chance to develop a variety of intriguing ideas. I returned to Bielefeld for one more month in the Fall of 2004 and it was only cluring this time that the final ideas presented in this thesis took shape. I am very proud to share co-authorship with Dr. Möller for a journal paper that describes a number of these ideas. Thanks also to Dr. Sonja Bisch-Knaden for the statistical analysis of the data in this paper. I am thankful also to new friends Wolfram Schenck, Frank Röben, Tim Köhler, and Angelika Deister for supporting my stay and making me feel quite at home.

My thanks to members of my thesis committee Dr. Nathalie Japkowicz, Dr. Tom Sherratt, and particular thanks to Dr. Gerhard Roth for a number of useful references and interesting discussions.

For financial support, I am most appreciative to NSERC for four years of funding and to Dr. Oppacher for help in the final year. Support for my first trip to Bielefeld was generously provided by the DAAD.

My parents have always trusted me enough to allow me to set my own course and for this - and so many other reasons-I am forever grateful. I am thankful also to my brother Adam and to my extended family, Nancy and the Inkpens. Thanls also to many friends and to the Perrys for their support.

Finally; for her undying support and encouragement. my deepest gratitude to my partner in life and love, Jill. 


\section{Contents}

Front Matter $\quad$ i

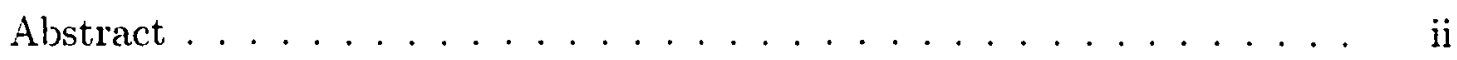

Acknowledgments ................. iv

Table of Contents ................... . vi

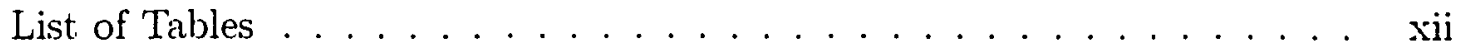

List of Figures . . . . . . . . . . . . . xiv

1 Introduction 1

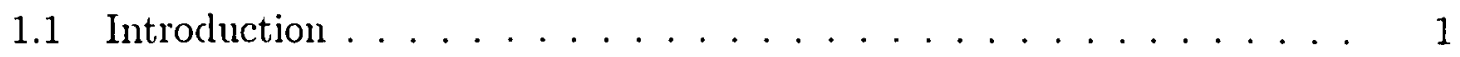

1.2 Insect Homing . . . . . . . . . . . . . . . . . 3

1.2 .1 Vision . . . . . . . . . . . . . . . 4

1.2 .2 Other Senses $\ldots \ldots \ldots \ldots \ldots \ldots \ldots \ldots \ldots \ldots \ldots$

1.2 .3 Navigation . . . . . . . . . . . . . . . . . 5

1.2 .4 Visual Homing . . . . . . . . . . . . . . .

1.2.5 The Snapshot Model . . . . . . . . . . . . . . . . . . . . 9 9

1.3 Robot Visual Homing . . . . . . . . . . . . . . . . . . . 14

1.3.1 Associative Homing . . . . . . . . . . . . . . . . 14

1.3 .2 Local Homing . . . . . . . . . . . . . . . . . 15

1.3 .3 Discussion . . . . . . . . . . . . . . . . 24

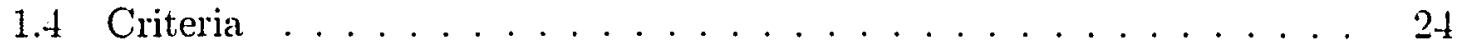

1.4 .1 Biological Plausibility . . . . . . . . . . . . . . 26

1.4 .2 Retinotopic Processing . . . . . . . . . . . 27

1.5 Research Question . . . . . . . . . . . . . . . 34

1.6 Outline of Thesis $\ldots \ldots \ldots \ldots \ldots \ldots \ldots \ldots \ldots$ 
2 Spherical Flow Fields $\quad 37$

2.1 Introduction . . . . . . . . . . . . . . . . . 37

2.2 Flow Fields in One Dimension . . . . . . . . . . . . . . . 38

2.2.1 Mathematical Development ............ . . 38

2.2.2 Example Flow Fields . . . . . . . . . . . . . . . . . . . . 41

2.3 Motion Fields in Two Dimensions . . . . . . . . . . . . . . . . 43

2.3.1 Mathematical Development ............. 43

2.3.2 Example Flow Field . . . . . . . . . . . . . . . 46

2.4 Conclusions ...................... . 48

3 Experiments and Methodology 49

3.1 Introduction . . . . . . . . . . . . . . . . . . . . . . 49

3.2 Image database . . . . . . . . . . . . . . . . . . . 49

3.3 Experiments . . . . . . . . . . . . . . . . . 52

3.3 .1 Assumptions . . . . . . . . . . . . . 52

3.3.2 Home Vectors for a Single Snapshot Position . . . . . . . . . . 54

3.3.3 Performance Metrics . . . . . . . . . . . . . 55

3.3.4 Performance Across Snapshot Position . . . . . . . . . . 58

3.3.5 Statistical Tests . . . . . . . . . . . . . . 61

3.4 Robot Homing Trials . . . . . . . . . . . . . . . . . . . . . 62

4 Other Methods $\quad 64$

4.1 Introduction . . . . . . . . . . . . . . . . . 64 64

4.2 Franz et al., 1998: Warping ............... 64

4.2.1 Equal Distance Assumption ... . . . . . . . . . . . 65

4.2.2 Parameterized Flow Fields . . . . . . . . . . . . 65

4.2 .3 Varping ...................... 66

4.2 .4 Pre-Processing ................. 67

4.2 .5 Tested Algorithm ................... 68

4.2 .6 Optimizing Parameters . . . . . . . . . . . . 69

4.2.7 Results on Image Database . . . . . . . . . . . . . . 71

4.3 Hafner, 2001: Centre-of-Mass ALV (COMALV) . . . . . . . . . . T7

4.3 .1 Extracting the Centre-Of-Mass ............ 80

4.3 .2 Tested Algorithm ................ 80 
4.3 .3 Optimizing Parameters . . . . . . . . . . . . . . . . so

4.3.4 Results on Image Database $\ldots \ldots \ldots \ldots$

4.4 Zeil et al., 2003: Gradient Descent. . . . . . . . . . . 84

4.4 .1 Outdoor Scenes . . . . . . . . . . . . . . 84

4.4 .2 Image Differencing . . . . . . . . . . . . . . 84

4.4.3 Gradients of Bielefeld Image Database . . . . . . . . . . 86

4.4.4 Homing by Gradient Descent . . . . . . . . . . . . . . 89

4.4 .5 Tested Algorithm . . . . . . . . . . . . . . . . . . . . . . . . . 91

4.4 .6 Results on Image Database $\ldots \ldots \ldots$. . . . . . . . 91

4.5 Comparative Results . . . . . . . . . . . . . . . . . . . 92

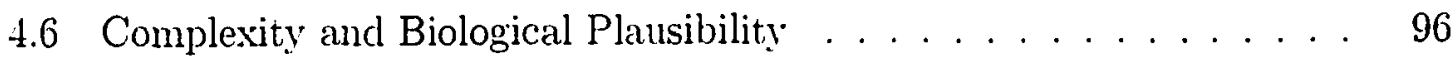

4.6 .1 Warping . . . . . . . . . . . . . . 96

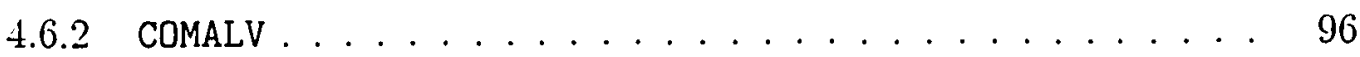

4.6 .3 GradDescent . . . . . . . . . . . . . . . 97

4.7 Conclusions $\ldots \ldots \ldots \ldots \ldots \ldots \ldots \ldots \ldots \ldots$

5 Vector Mapping $\quad 99$

5.1 Introduction . . . . . . . . . . . . . . . . . . . . . . . . 99

5.2 Notation . . . . . . . . . . . . . . . . . . . . . . . 99

5.3 Hong et. al's 1-D Technique . . . . . . . . . . . . . . . 100

5.3 .1 Correctness . . . . . . . . . . . . . . . . 102

5.4 Vardy \& Möller's 2-D Technique . . . . . . . . . . . . 103

5.4 .1 Computing the Final Home Vector . . . . . . . . . 107

5.4 .2 Correctness . . . . . . . . . . . . . . 108

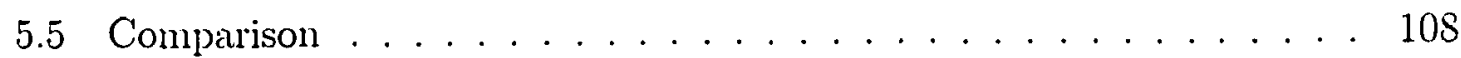

6 CGSM 111

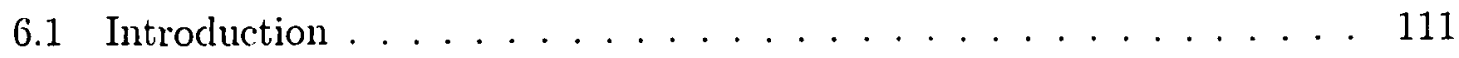

6.2 Assumption . . . . . . . . . . . . . . . . . . . . . 112

6.3 Overview of Processing . . . . . . . . . . . . . . . . . 112

6.4 Describing a Retinotopic Natrix . . . . . . . . . . . . 114

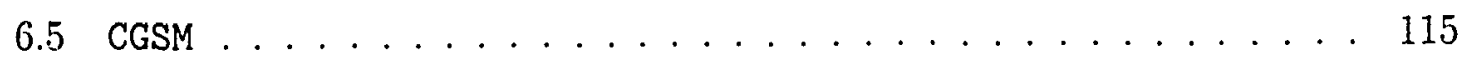

6.5 .1 Gaussian Filter . . . . . . . . . . . . . . . 115

6.5.2 Harris Corner Detector . . . . . . . . . . . . . . 116

viii 
6.5.3 Gaussian Filter (Stable) . . . . . . . . . . . . . . . 117

6.5.4 Local Maxima Detection and Thresholding . . . . . . . . . 118

6.5.5 The Potential Surface . . . . . . . . . . . . . . . . . . 119

6.5.6 The Weighted Gradient of the Potential Surface . . . . . . . . 121

6.5.7 Calculating the Home Vector . . . . . . . . . . . . . . 121

6.5.8 Order of Matching . . . . . . . . . . . . . . . . . . 122

6.6 Optimizing Parameters . . . . . . . . . . . . . . . . . . . . . 122

6.7 Results on Image Database . . . . . . . . . . . . . . . . . . 124

6.8 Comparative Results . . . . . . . . . . . . . . . . . 127

6.9 Robot Homing Trials . . . . . . . . . . . . . . . . . . . . . . . 128

6.10 Analysis . . . . . . . . . . . . . . . . . . . . . 129

6.11 Complexity and Biological Plausibility . . . . . . . . . . 132

6.12 Conclusions . . . . . . . . . . . . . . . . 134

7 Scale Invariant Image Descriptors 135

7.1 Introduction . . . . . . . . . . . . . . . . 135

7.2 Scaling Nodel . . . . . . . . . . . . . . . . . . . 136

7.3 The Scale Invariant Descriptor . . . . . . . . . . . . . . . 137

7.3.1 Conditions . . . . . . . . . . . . . . . . . . 139

7.3 .2 Structure . . . . . . . . . . . . . . . . 139

7.4 SIMatch . . . . . . . . . . . . . . . . . . . 139

$7.5 \quad$ SIPair . . . . . . . . . . . . . . . . . 141

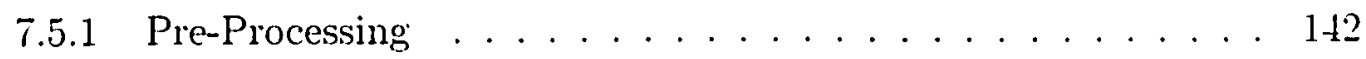

7.6 Optimizing Parameters . . . . . . . . . . . . . . 143

7.7 Results On Image Database . . . . . . . . . . . . . . . 143

7.7.1 Sample Flow Fields . . . . . . . . . . . . . . . . . 143

7.7.2 Dot Product Images $\ldots \ldots \ldots \ldots$. . . . . . . . . . . . . . .

7.8 Comparative Results . . . . . . . . . . . . . 150

7.9 Analysis . . . . . . . . . . . . . . . . 154

7.10 Complexity and Biological Plausibility . . . . . . . . . 156

7.10 .1 The Scale Invariant Descriptor . . . . . . . . . . . 156

7.10 .2 siMatch . . . . . . . . . . . . . . 15s

7.10 .3 SIPair . . . . . . . . . . . . . . . . . 159

7.11 Conclusions . . . . . . . . . . . . . . . 159 
8 Towards Simpler Matching Methods 161

8.1 Introduction . . . . . . . . . . . . . . . . . . 161

8.2 BlockMatch . . . . . . . . . . . . . . . . . . 162

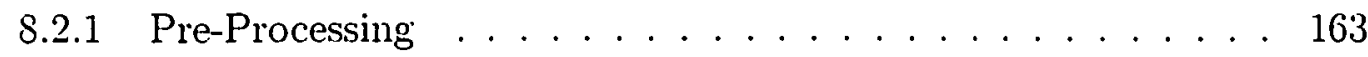

8.2.2 Optimizing Parameters . . . . . . . . . . . . . . . . . 163

8.2.3 Results on Image Database . . . . . . . . . . . . . . 164

8.2.4 Comparative Results . . . . . . . . . . . . . . . . . . 166

8.2 .5 Robot Homing Trials . . . . . . . . . . . . . . . . 168

8.2 .6 Analysis . . . . . . . . . . . . . . . . 170

8.3 IntMatch and GradMatch $\ldots \ldots \ldots \ldots \ldots \ldots \ldots$

8.3.1 Pre-Processing . . . . . . . . . . . . . . . 175

8.3.2 Optimizing Parameters . . . . . . . . . . . . . . . 175

8.3.3 Results on Image Database . . . . . . . . . . . 176

8.4 Comparative Results . . . . . . . . . . . . . . . 178

8.5 Complexity and Biological Plausibility . . . . . . . . . 181

8.5.1 BlockMatch . . . . . . . . . . . . . . . . 181

8.5.2 IntMatch and GradMatch . . . . . . . . . . . . . 181

8.6 Conclusions . . . . . . . . . . . . . . . . 182

9 Differential Methods 184

9.1 Introduction . . . . . . . . . . . . . . . . 184

9.2 Differential Techniques . . . . . . . . . . . . . . 185

9.2 .1 Firstorder . . . . . . . . . . . . . . . . 185

9.2 .2 Secondorder . . . . . . . . . . . . . . 187

9.3 Assumptions . . . . . . . . . . . . . . . . . 188

9.4 Approximate Vector Mapping . . . . . . . . . . . . . . . . . . . . 189

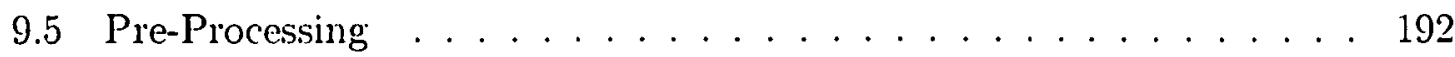

9.6 Optimizing Parameters . . . . . . . . . . . . . . . . . . . . . . . . . 192

9.7 Results on Image Database . . . . . . . . . . . . . . . . . . . 192

9.8 Comparative Results . . . . . . . . . . . . . . . . . . . . . . 196

9.9 Analysis . . . . . . . . . . . . . . . . . . . . . . 197

9.10 Complexity and Biological Plausibility . . . . . . . . . . . . 201

9.11 Conclusion . . . . . . . . . . . . . . . . . . 201 
10 Conclusions, Contributions, and Future Research 204

10.1 Introduction . . . . . . . . . . . . . . . . . 204

10.2 Conclusions . . . . . . . . . . . . . . . . . 204

10.2.1 Primary Conclusion . . . . . . . . . . . . . . . . . 204

10.2.2 Other Major Conclusions . . . . . . . . . . . . . . 205

10.3 Contributions . . . . . . . . . . . . . . . 206

10.4 Future Research . . . . . . . . . . . . . . . . . . 208

10.4 .1 Orientation . . . . . . . . . . . . 208

10.4 .2 Filtering . . . . . . . . . . . . . . . . 209

10.4.3 Neural or Evolutionary Optimization . . . . . . . . . . . 209

10.4.4 Hybrid Approaches . . . . . . . . . . . . . . . . . . . 209

10.4.5 Relaxing Motion Constraints . . . . . . . . . . . . . 210

10.4 .6 Biology . . . . . . . . . . . . . . . . 210

10.4.7 Long-Range Homing . . . . . . . . . . . . . 211

10.5 Summary . . . . . . . . . . . . . . . . . . 212

A Scale Invariant Image Descriptors $\quad 213$ 


\section{List of Tables}

1.1 Reviewed approaches to local homing. . . . . . . . . . . . . 25

3.1 Values of averaged performance metrics for the demonstration methods. 60

4.1 Statistical significance of the difference in $A A E_{*}$ and $R R_{*}$ between homing methods. . . . . . . . . . . . . . 94

4.2 Statistical significance of the difference in $A A E_{*}\left(\right.$ hall1) and $R R_{*}($ hall1) between homing methods. . . . . . . . . . . . . 94

4.3 Statistical significance of the difference in $A A E_{*}\left(\right.$ hal12) and $R R_{*}$ (hal12) between homing methods. . . . . . . . . . . . . 95

4.4 Statistical significance of the difference in $A A E_{*}(*, *)$ and $R R_{*}(*, *)$ between homing methods for all pairings of image collections from the office environment. . . . . . . . . . . . . . . . . . . 95

6.1 Statistical significance of the difference in $A A E_{*}$ and $R R$, between Warping and CGSM. . . . . . . . . . . . . . . . 128

7.1 Statistical significance of the difference in $A A E_{*}$ (original) and $R R_{*}$ (original) between homing methods. . . . . . . . . . . . . . 152

7.2 Statistical significance of the difference in $A A E_{*}$ (hall1) and $R R_{*}$ (hall1) between homing methods. . . . . . . . . . . . . . . 153

7.3 Statistical significance of the difference in $A A E_{*}\left(\right.$ hall2) and $R R_{*}$ (hall2) between homing methods. . . . . . . . . . . . . . 153

7.4 Statistical significance of the difference in $A A E_{*}(*, *)$ and $R R_{*}(*, *)$ between homing methods for all pairings of image collections from the office environment. . . . . . . . . . . . . . . . . . . 15-

xii 
8.1 Statistical significance of the difference in $A A E_{*}$ and $R R_{*}$ between Warping and BlockMatch. . . . . . . . . . . 167

8.2 Statistical significance of the difference in $A A E_{*}$ (original) and $R R_{*}$ (original) between homing methods. . . . . . . . . . . . . . . . 179

8.3 Statistical significance of the difference in $A A E_{*}$ (hall1) and $R R_{*}$ (hall1) between homing methods. . . . . . . . . . . . . . . . 179

8.4 Statistical significance of the difference in $A A E_{*}\left(\right.$ hall2) and $R R_{*}($ hall2) between homing methods. . . . . . . . . . . . . . . 180

8.5 Statistical significance of the difference in $A A E_{*}(*, *)$ and $R R_{*}(*, *)$ between homing methods for all pairings of image collections from the office enviromment. . . . . . . . . . . . . . . . . 180

9.1 Statistical significance of the difference in $A A E_{*}$ (original) and $R R_{*}$ (original) between homing methods. . . . . . . . . . . . . . . . . . 198

9.2 Statistical significance of the difference in $A A E_{*}\left(\right.$ hall2) and $R R_{*}$ (hall2) between homing methods. . . . . . . . . . . . . . . . . 199

9.3 Statistical significance of the difference in $A A E_{*}(*, *)$ and $R R_{*}(*, *)$ between homing methods for all pairings of image collections from the office environment. . . . . . . . . . . . . . . . . . . . . 199 


\section{List of Figures}

1.1 The snapshot model. . . . . . . . . . . . . . . . . . . . . . . 13

1.2 The ALV model. . . . . . . . . . . . . . . . . . . . . . . 19

1.3 The neural snapshot model. . . . . . . . . . . . . . . . 22

1.4 The optic lobe of the bee. . . . . . . . . . . . . . . . 29

2.1 Motion of agent from $\mathbf{s}$ to $\mathbf{c}$ and the resultant flow of landmark 1. . . 39

2.2 Motion of an agent within two different environments. . . . . . . 4

2.3 Motion of agent from $\mathrm{s}$ to $\mathrm{c}$ and the resultant flow of landmark $\mathrm{l}^{\prime}$ within

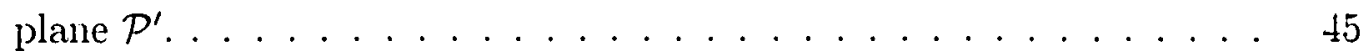

2.4 Outside perspective view of the sphere visualized in OpenGL. . . . . 46

2.5 Panoramic images taken from within a sphere at $\mathbf{s}(\mathrm{a})$ and $\mathbf{c}(\mathrm{b})$, and the correspondence vectors between them $(\mathrm{c}) \ldots \ldots \ldots \ldots \ldots+7$

3.1 (a) The ActivMedia Pioneer 3-DX robot with attached camera and hyperbolic mirror. (b) A close-up of the camera and hyperbolic mirror

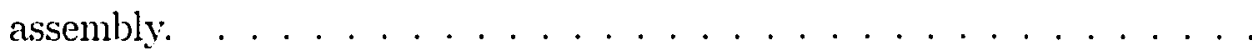

3.2 Sample images from eight image collections in the office environment. Images taken from position $(3,0) \ldots \ldots \ldots \ldots \ldots \ldots$

3.3 Sample images from two image collections in the hall environment. Images taken from position $(5,10) \ldots \ldots \ldots \ldots$. . . . . . . . . 54

3.4 Home vector fields for the ideal homing method and two noisy methods for snapshot position $(6,4)$ of image collection original. . . . . . .

3.5 Homing attempts computed for the home vectors in figure $3.4(\mathrm{~b})$ with varying settings for $l_{\mathrm{rr}} \ldots \ldots \ldots \ldots \ldots \ldots \ldots$

3.6 Positions of images, snapshots, robot snapshot positions, and robot start positions within the office environment capture grid. . . . . . . . 
4.1 On the top is image $(6,8)$ from image collection original. . . . . . 68

4.2 Demonstration of Warping with a small search space. . . . . . . . 70

4.3 Home vector fields for Werping ( $a, b)$, COMALV $(c, d)$, and GradDescent $(\mathrm{e}, \mathrm{f})$, for snapshot positions $(6,4)(\mathrm{a}, \mathrm{c}, \mathrm{e})$ and $(0,16)(\mathrm{b}, \mathrm{d}, \mathrm{f})$ in image collection original. . . . . . . . . . . . . . 72

4.4 $A A E$ and $R R$ across snapshot positions for all methods on image col-

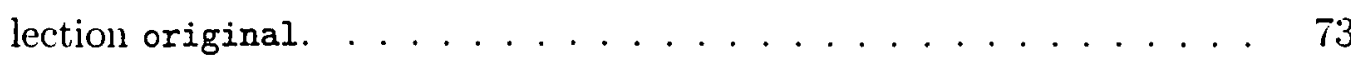

4.5 Home vector fields for Warping ( $a, b)$, COMALV (c,d), and GradDescent $(\mathrm{e}, \mathrm{f})$, for snapshot position $(3,12)$ in image collections hall1 and hall2. 75

4.6 Home vector fields for Warping and COMALV that warrant special at-

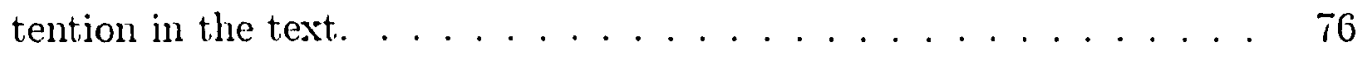

4.7 $A A E$ and $R R$ across snapshot positions for all methods on image collection hall1. ................. Ti

4.8 $A A E$ and $R R$ across snapshot positions for all methods on image col-

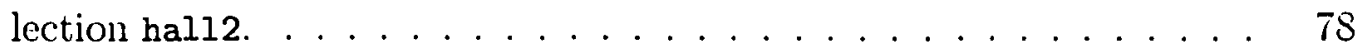

$4.9 A A E_{\text {t }}$ and $R R$, orer all pairings of the eight office image collections. . 79

4.10 Average landmark vector fields as computed by CoMALV. . . . . . . . 83

4.11 SSD between each of 20 snapshot images and all of the remaining 169 images of original. . . . . . . . . . . . . 87

4.12 $\mathrm{SSD}^{-4}$ between four snapshot positions along the bottom boundary of original and all other images from that collection.

4.13 SSD between image $(3,4)$ of original and rotated versions of images $(3,4),(0,0),(9,0),(0,16)$, and $(9,16) . \ldots \ldots \ldots$

4.14 (a) SSD between image $(3,4)$ of original and rotated versions of all other images from the top portion of the image database. . . . . . 9 90

5.1 Illustration of the main idea behind Hong et al.'s vector mapping technique. . . . . . . . . . . . . . . . . . . 100

5.2 Illustration of the direction of home vector $\mathbf{u}_{\mathrm{hong}}$ as computed by Hong et al.'s vector mapping technique. . . . . . . . . . . . . . 102

5.3 Motion of an agent from $\mathrm{s}$ to $\mathrm{c}$ and the resultant change in viewing position of a landmark $1 . \ldots \ldots \ldots$. . . . . . . . . 104

5.4 Oblique view of plane containing $\mathbf{s}$ and $\mathbf{c} \ldots \ldots \ldots \ldots . \ldots 105$ 
5.5 Performance of three techniques for visual homing for the sphere environment introduced in chapter $2 \ldots \ldots$. . . . . . . . . 110

6.1 Processing applied by CGSM. . . . . . . . . . . . . . . 113

6.2 Implementation of Gaussian filter in a retinotopic matrix. . . . . . . 116

6.3 Part of the implementation of the Harris corner detector in a retinotopic matrix. . . . . . . . . . . . . . . 117

6.4 Implementation of local maxima detection and thresholding in a retinotopic matrix. . . . . . . . . . . . . . . . . 118

6.5 Development of potential surface over five iterations with $n_{p s}=v_{p s}=10.120$

6.6 Processing applied br reversed CGSM. . . . . . . . . . . . . . 123

6.7 Home vector fields for Warping $(a, b)$ and $\operatorname{CGSM}(c, d)$ for snapshot positions $(6,4)(a, c)$ and $(0,16)(b, d)$ in image collection original. . . . 125

6.8 $A A E$ and $R R$ across snapshot positions for all methods on image collection original. . . . . . . . . . . . . . 125

6.9 Home vector fields for Warping ( $a, b)$ and CGSM (c,d), for snapshot position $(3,12)$ in image collections hall1 and hall2. . . . . . . 126

6.10 AAE and RR across snapshot positions for all methods on image collection hall1. . . . . . . . . . . . . . . . . . 126

6.11 $A A E$ and $R R$ across snapshot positions for all methods on image col-

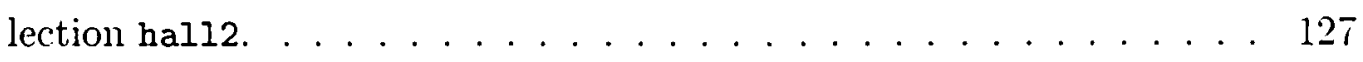

6.12 $A A E_{*}$ and $R R$, over all pairings of the eight office image collections. . 12T

6.13 Performance of Warping and CGSM when tested live on the Pioneer robot.130

6.14 Number of features detected by CGSM with parameters as given in sec-

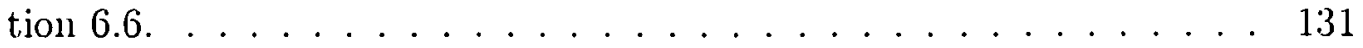

6.15 Plot of $A A E$, versus threshold value for image collections original. hall1, and hall2. . . . . . . . . . . . . 133

7.1 Scaling of an image $I$ and the value of $I$ along a channel. . . . . . . 137

7.2 Retinotopic matrix to compute g. . . . . . . . . . . . . 140

7.3 Example flow field for snapshot position $(6,4)$ and current positions

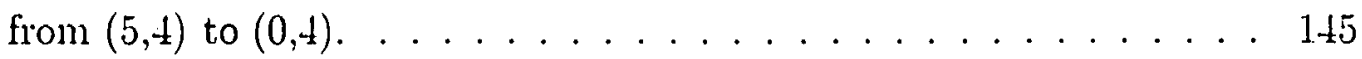

7.4 Variation in $D P(\mathbf{p}, \mathbf{p})$ for SIPair. . . . . . . . . . 146 
7.5 Home vector fields for Warping (a,b), SIMatch (c,d), and SIPair (e,f), for snapshot positions $(6,4)(a, c, e)$ and $(0,16)(b, d, f)$ in image collection original.

7.6 $A A E$ and $R R$ across snapshot positions for all methods on image collection original. . . . . . . . . . . . . . 148

7.7 Home vector fields for Warping (a,b), SIMatch (c,d), and SIPair (e,f) for snapshot position $(3,12)$ in image collections hall1 $(a, c, e)$ and hall2 $(b, \mathrm{~d}, \mathrm{f})$

7.8 $A A E$ and $R R$ across snapshot positions for all methods on image collection hall1.

7.9 $A A E$ and $R R$ across snapshot positions for all methods on image col-

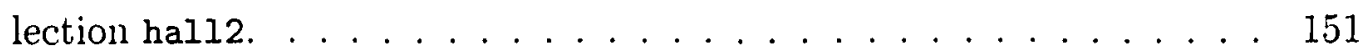

7.10 $A A E$, and $R R$, over all pairings of the eight office image collections. . 152

7.11 Dot product image (c) for snapshot position $(3,12)$ and current position

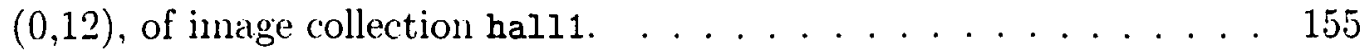

7.12 Plots of $A . A E_{*}$ (original) vs. parameters. . . . . . . . 157

S.1 Home vector fields for Warping $(\mathrm{a}, \mathrm{b})$ and BlockMatch $(\mathrm{c}, \mathrm{d})$, for snapshot positions $(6,4)$ (a.c) and $(0,16)$ (b.d) in image collection original. 164

8.2 $A . A E$ and $R R$ across snapshot positions for all methods on image col-

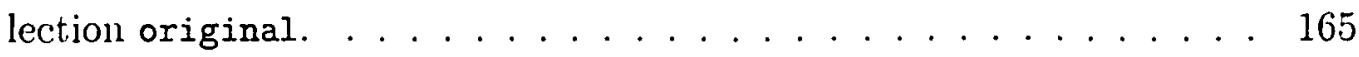

8.3 Home vector fields for Warping ( $a, b)$ and BlockMatch (c,d), for snapshot position $(3,12)$ in image collections hall1 (a,c) and hall2 (b,d). .

8.t $A A E$ and $R R$ across snapshot positions for all methods on image collection hall1. . . . . . . . . . . . . . . 16

8.5 $A A E$ and $R R$ across snapshot positions for all methods on image col-

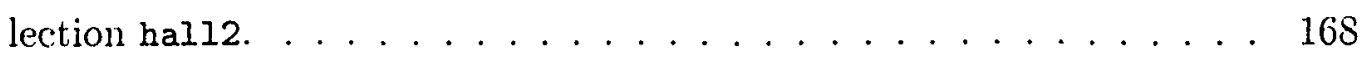

8.6 AAE* and $R R$, over all pairings of the eight office image collections. . 169

8.7 Performance of BlockMatch when tested live on the Pioneer robot. . 169

S.8 Example flow field for snapshot position $(6,-4)$ and current position $(5,4) .171$

8.9 Flow fields for-snapshot position $(6,4)$ and current positions from $(4,4)$

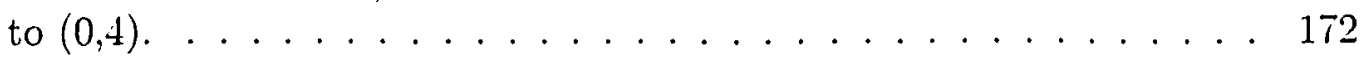

8.10 Plots of angular error vs. parameters. . . . . . . . . . 173

xvii 
8.11 The effects of increased Gaussian filtering on image $(6,4)$ from image collection original. . . . . . . . . . . . . . . . . 174

8.12 Home vector fields for IntMatch ( $a, b)$ and GradMatch (c,d), for snapshot positions $(6,4)(a, c)$ and $(0,16)(b, d)$ in image collection original. 176

8.13 Home vector fields for IntMatch (a,b) and GradMatch (c,d), for snapshot position $(3,12)$ in image collections hall1 and hal12. . . . . . 177

9.1 Illustration of the robot coordinate system and the vectors involved in the approximate vector mapping technique. . . . . . . . . . . . 190

9.2 Home vector fields for block matching $(a, b)$ and warping $(c, d)$, for snapshot positions $(6,4)(a, c)$ and $(0,16)(b, d)$ in image collection original. 193

9.3 $A A E$ and $R R$ across snapshot positions for all methods on image collection original. . . . . . . . . . . . . . . . 194

9.4 Home vector fields for Warping ( $a, b)$ and BlockMatch $(c, d)$, for snapshot position $(3,12)$ in image collections hall1 (a,c) and hall2 (b,d). 195

9.5 $A A E$ and $R R$ across snapshot positions for all methods on image collection hall1. . . . . . . . . . . . . . . . 196

9.6 $A A E$ and $R R$ across snapshot positions for all methods on image collection hall2. . . . . . . . . . . . . . . . . . . . . . .

9.7 $A A E_{*}$ and $R R_{*}$ over all pairings of the eight office image collections. . 198

9.8 Error images for both clifferential methods at snapshot position (6.8) and current position $(5.8) \ldots \ldots \ldots \ldots \ldots \ldots$

9.9 Average error images and one-dimensional plots for both differential methods on image collection original. . . . . . . . . . . 202 


\section{Chapter 1}

\section{Introduction}

\subsection{Introduction}

Insects are capable of incredible feats of navigation. Despite small brains and low visual acuity; foraging insects such as bees and ants are able to return to their nests and to feeding sites from great distances. Travel distances are "of the order of a million times their body length" [106]. This level of performance stands in stark contrast to the level of current robot navigators. Consider a typical robot with a body length of $10 \mathrm{~cm}$. If this robot were to venture out for a distance equal to just one million times its body length, it would have travelled $100 \mathrm{~km}$. The notion of a robot being able to return to a goal from as far away as $100 \mathrm{~km}$ is not only outside the realm of testability; it is far outside the realm of possibility for any known robot navigator. Yet insects are typified as 'simple' creatures, with only on the order of one million neurons. Therefore the mechanisms they employ might be both simple enough for biologists to elucidate, and for engineers to duplicate.

Navigation, however, is a large topic. The study of insect navigation is subdivided according to the different strategies and modalities that insects appear to employ [12]. Two of the primary modalities are path integration (i.e. dead reckoning) and the use of visual landmarks. One important use of visual landmarks is to allow the insect to home in to a goal after arriving in the goal's general vicinity: We refer to this ability as visual homing. This thesis will study methods for visual homing that might plausibly be implemented in the brain of an insect.

These methods will be tested on images from a mobile robot. Thus, this work also 
pertains to the subject of robot navigation. A variety of approaches to robot navigation have been posited ${ }^{1}$. Traditional approaches focused on developing and maintaining an accurate geometric map (see review by Kuipers and Byun [56]). Within this framework, homing to a goal location would be achieved by localizing both the goal and the current location within the map and planning a route between them. However, to update the map requires the solution of the simultaneous localization and mapping (SLAN) problem [63]. Also, accurately registering elements of the map with features of the world is a notoriously difficult problem [5]. A focus on homing obviates the need of a map and changes the nature of the problem. Whereas traditional approaches demand an answer to the question "Where am I?", homing requires only. an answer to the question "How do I reach the goal?" [23]. A variety of methods have been proposed for robot visual homing, many of which take inspiration from visual homing in animals.

The broad approach which this work falls under is known variously as biorobotics [103] or biomimetics. The underlying notion is that animals and autonomous robots must both solve similar problems. Therefore, the study of how a problem is solved in one type of agent (animal or robot) can assist the study of the same problem in the other type of agent. From the perspective of robotics, biology can inspire good technical solutions. The extent to which this inspiration impacts a robot's design will depend entirely on its utility: Thus, biological inspiration does not imply any form of constraint. On the other hand, a particular robot control algorithm will be of no interest to biology unless it can be shown to be plausible as a model of some animal behaviour. We define biological plausibility as the likelihood of an algorithm existing in biology: For an algorithm to be biologically plausible it must be constrained to share some set of properties known to exist in the model animal. The constraint. employed here is that a homing method must be implementable within a matrix of processing elements arranged in a retinotopic fashion. The term 'retinotopic' means that the spatial arrangement of cells on the retina is maintained through subsequent layers of processing. Retinotopic organization is found in visual systems throughout the animal kingdom and is particularly prominent in insects.

The homing methods developed in the course of this thesis will be assessed firstly by their performance. Ineffective homing methods would be of little help to biologists

\footnotetext{
${ }^{1}$ Approaches that require the environment to be modified, or for which a map of the enviromment is provided, will not be considered.
} 
attempting to explain the highly-effective navigational abilities of insects. Performance will be measured primarily on a database of images collected from a variety of different enviromments under varying conditions. Some of these results will be validated by online tests on the mobile robot used to capture the image database. Mounted above the camera on this robot is a hyperbolic mirror which allows $360^{\circ}$ panoramic images to be captured. Insects such as honeybees have near panoramic vision and the low resolution of their compound eyes is also reflected in the images used here. Statistical tests will be used to evaluate the difference between methods and Franz et al.'s [26] method will be used for comparison.

The next section will review insect visual homing as well as the place of homing within insect navigation. A review of robotic approaches to visual homing will follow. After this a cliscussion of the criteria to be employed for methods in this thesis will be presented. This section will include particular background detail on retinotopic processing. Following this, the research question will be presented and a summary of the thesis provided.

\subsection{Insect Homing}

Bees forage for pollen and nectar over immense tracts of terrain and return with their spoils to the nest. Euglossine bees have been reported to return to their nests after being displaced by up to $23 \mathrm{~km}$. These same bees have been observed to follow consistent foraging routes. For example, a single bee was observed to follow the same route between a sequence of flowers on five subsequent mornings with each leg of the journey between these flowers being $70 \mathrm{~m}$ or greater in length [50]. Ants too exhibit highly impressive navigational performance. The desert ant Cataglyphis fortis embarks on foraging trips hundreds of metres in length [106]. Of course, insects are not the only navigators in the animal kingdom. The navigational abilities of birds and mammals are also quite astonnding (see various papers in [41]). Indeed, Morris provided a very well-known illustration of visual homing in rats [78]. However, insects have a number of advantages as experimental subjects and the picture of navigation in insects appears to be more complete than for other animals. Also, as Collett and Collett put it "Insects have far fewer neural resources than vertebrates, so data from insects might be useful in revealing the essential components of efficient navigation" 
$[10]$.

It is postulated that insects achieve their navigational feats by combining a number of strategies and special senses. Visual homing is just one such strategy and it is necessary to first describe other pertinent aspects of insect navigation before delving into visual homing. We begin with brief depictions of insect vision and other special senses. The emerging picture of insect navigation is then discussed. At this point we will review research that specifically addresses visual homing.

\subsubsection{Vision}

The compound eyes of insects are composed of an array of light-absorbing receptors. Above each receptor is a lens that focuses light onto the receptor. The field of view for receptors is on the order of $2^{\circ}$ and the angular separation between them is also about $2^{\circ}$ [46]. The angular separation between facets of a bee's eye can be as small as about $1^{\circ}$, vet the angular separation between receptors in the human eve is about 60 times smaller [59]. Thus, insect vision operates at a much lower resolution than human vision. Also, insects have fixed optics and cannot use either lens accommodation or eye convergence to estimate the distance of viewed objects. Further, the small separation between insect eyes means that depth perception by stereo disparity is possible only for very nearby objects [91]. For these reasons the passive perception of depth can be ruled out as a cue for insect navigation.

Yet insect eyes have properties not possessed by human eyes. While the spatial resolution of insect eyes is low, their temporal resolution is high meaning that insect visual systems are less subject to motion blur. Of particular interest here is the fact that insect eyes sample such a large proportion of the visual field. Honerbee eyes have a horizontal field of view of about $310^{\circ}$ [59]. Panoramic vision 2 has a number of possible advantages. For navigation it allows the same set of landmarks to be perceptible over a large area. For a motion consisting only of translation, a panoramic image will reveal two special regions called the focus of contraction and focus of expansion where the apparent motion will be small (these terms will be defined in chapter 2). If a motion consists also of rotation then panoramic vision enables the component of rotation to be separated from the component of translation [79].

\footnotetext{
${ }^{2}$ We will generally use the term panoramic to indicate very wide field vision (usually $360^{\circ}$ ).
} 


\subsubsection{Other Senses}

Insects have alternate sensory modalities in addition to vision, touch, smell, hearing, and taste. One such modality in bees and ants is the skylight compass. The upper portion of the eyes of these insects are specially equipped to detect the polarization pattern of light in the sky and to infer the animal's orientation from this information [106]. Another modality known in honevbees is the mannetic compass. Honeybees trained to search for a reward can be manipulated in their preferred orientation while searching by use of an artificially imposed magnetic field [9]. Thus, honeybees have two available compass systems. Another modality; well-known in ants, is the ability to lay and detect pheromone chemical trails and to use these trails to mark routes to food and nest-sites. Bees, as flying insects, cannot employ this strategy and there are also species of ants which do not use pheromone trails. Some of the experimental results on ants that we will be referring to were found on Cataglyphis which cloes not use pheromone trails, likely because of the extreme temperature of its environment wherein such chemicals would swiftly evaporate [106].

\subsubsection{Navigation}

The emerging consensus on insect navigation is that it is enabled by a combination of special purpose capabilities (see reviews $[105,12,10]$ ). Two of the most important capabilities are path integration and the use of visual landmarks.

\section{Path Integration}

Path integration, known in nautical navigation as dead reckoning, is the use of orientation and odometry information to determine position relative to some reference frame. Experiments on Cataglyphis provide the prototypical example of path integration in insects. Cataglyphis forages out from its nest in search of dead arthropods to return with. Its outbound route may appear quite winding and convoluted, vet upon finding food it will not retrace this winding route back home but will embark on a direct return path. The theory is that such insects integrate the angles and distances over the outbound path into a single home vector which is continuously updated to point back home. If, upon finding food an ant is picked up by the experimenter and transported in a closed container to another location, it will immediately begin to 
head back in the direction where its nest would have been had it not been disturbed. Moreover, it will travel in this direction for a distance approximately equal to the actual distance to the nest at the point where it was picked up. This is known because after this distance has been covered it will immediately begin to roam about in a characteristic search pattern. Computing a home vector requires orientation information that is provided by one or both of the insect's compass systems (skylight and/or magnetic). This computation also requires the ability to judge distances travelled which is thought to be determined from the rate of retinal image flow that the insect experiences $[92,106]$. Path integration is extremely important in insect navigation, yet it cannot be the only system involved. By its nature it is subject to cumulative error and it is believed to be insufficiently precise to locate places as small as a single flower or the entrance to a nest [6]. It seems likely that this error would be increased dramatically for flying insects, given the buffeting of winds and other such factors.

\section{Visual Landmarks}

To supplement the path integration system it is generally thought that insects make use of visual landmarks. One first simple picture on the relationship between path integration and landmarks would be as follows. An insect returning from a foraging trip follows its home vector back to the vicinity of its nest. Even when the home vector has been completely retraced, the insect will likely still be at some distance away from the nest. This is due to cumulative errors in the path integration system. The nest itself may be indistinguishable from the surroundings (presumably so as to avoid predation). However, we presume that on its initial departure from the nest it had stored some representation of the array of landnarks surrounding the nest. Thus, the insect can now use the disparity between the landmarks currently viewed and the stored landmarks to compute a home vector. That is, the insect uses visual homing to pinpoint the nest after arriving within its general vicinity: On this account, path integration provides the main navigation system with landmarks being used for only the final stage. This depiction is equally applicable for the foraging insect who wishes to return to a particular food source. If an approximate vector to the food source is obtained and the insect had previously stored some representation of the landmarks around the food source, then the insect could home to the food just as it homed to the nest. 
The general picture given above becomes more complicated when we consider that the route of an insect back to its nest, or to some other site, may be quite long and that landmark information may be needed en route to the goal as well. Collett [8] identifies a number of different ways in which insects use landmarks along the route to a goal. Ants are known to take biased detours consistently to the right or left of particular landmarks along a familiar route. Both bees and ants make direct approaches to large prominent landmarks lying on or near the path to a goal. Since the time of Collett's review, a variety of other uses for landmarks en route have been discovered. For example, it has been found that ants will use an extended landmark such as a parallel wall to guide them towards a food source. Experiments have shown that two distinct species of ant achieve this guidance by maintaining the top of the wall at a fixed retinal position [85, 31]. Moving so as to restore landmarks to particular retinal positions is one of two major approaches to visual homing and will be discussed in more detail below.

Collett [8] also discusses the hypothesis that insects form associations between vectors and scenes. For example, upon arriving at a food source an insect might associate the home vector from its path integration system with the local scene. This would aid the insect in returning home from the current position on subsequent visits. The association of scenes and vectors might also enable complex foraging routes, such as those known to be followed by bees [50]. Support for this view has been provided through the experiments of Menzel et al. [68]. On the other hand, Wehner et al. [106] argues that this hypothesis is unwarranted. He postulates that insects acquire sequences of images in memory. Visual homing is then applied in succession. Reaching. a particular place in the environment will trigger the recall of a new image. The insect then homes to the location where this new image was taken. This accounts for the observed routes that bees follow so characteristically:

\subsubsection{Visual Homing}

Two views have emerged on the basic mechanism of visual homing in insects. Möller [73] defines these as the template and parameter hypotheses. The template hypothesis assumes that an image taken from the goal is stored as the representation for that position. This image is fixed at the retinal positions at which it was originally stored. The parameter hypothesis assumes that some set of parameters is extracted and 
stored as the representation for the goal position. Möller also points out that the same two hypotheses have been proposed to explain pattern recognition in insects.

\section{The Template Hypothesis}

The template hypothesis is typified by Cartwright and Collett's [6] model, which has come to be known as the snapshot model (this model will be reviewed in depth in the next section). The snapshot model is a computational model designed to produce results similar to that observed in Cartwright and Collett's experiments on honeybees trained to a food source. These authors propose that a homing bee searches for the food source "...where the compass bearings of the landmarks on its retina were the same as they had been when it was stationed at the food source". The snapshot model is an image matching procedure that moves the agent to reduce discrepancies between the retinotopically fixed snapshot image and the current image. Evidence for image matching has accumulated for a variety of species including hoverflies [11], water striders [53], fruit flies [16], and wood ants [52]. As mentioned above, image matching has also been implicated for landmark guidance en route to a goal in two species of ant $[85,31]$.

Minimally, image matching requires some features in common between the current and snapshot images. In order for image matching to be successful it is also necessary. for the discrepancies between images to be reduced as much as possible. This can be achieved by viewing the goal environment from a consistent orientation. As described above, insects such as bees and ants are able to infer orientation from the polarization pattern of light in the sky; as well as by detecting the local magnetic field. While homing, it is therefore possible for them to restore their orientation to that held when capturing the snapshot image. This might be achieved only for particular moments during homing, or throughout a homing episode. Honeybees are known to take up characteristic orientations when close to a goal, such as facing magnetic south [9]. This would free them from the requirement of associating particular orientations with particular goals. Also, it has been shown that ants cannot internally rotate an image and therefore must view the scene from the same body orientation in order to visually home [106]. Bees and wasps will perforn a characteristic learning flight when leaving a goal. It has been observed that when returning to the goal they will tend to restore the orientations held during the learning flight [109]. All of the above suggests 
that the image matching methods used by insects are based on the assumption that the orientation held at the goal is recovered to support homing.

Some more recent evidence suggests that a different requirement on orientation may exist for certain species of ant. Analogous to the learning flights of bees and wasps referred to above, ants perform learning walks. Nicholson et al. [82] found that ants departing a newly discovered food site will turn back and look at landmarks surrounding the feeder. With decreasing frequency as the ant moves away from the site, ants will turn about and fixate landmarks on their frontal retina. On subsequent returns the ant will again fixate landmarks. Nicholson et al. propose that ants may perform what Möller [73] refers to as partial image matching, whereby the ant moves so as to match one landmark at a time with a snapshot view of that landmark. This proposal suggests that ants may store multiple snapshot images for a single goal-one per landmark. Graham et al. [30] argue that these separate snapshot images may have associative links between them such that they form an 'extended snapshot'.

\section{The Parameter Hypothesis}

The parameter hypothesis is supported by a far smaller number of studies. It is typified by Anderson's [1] model which was developed from experiments on honeybees trained to search for food at the centre of a circle of eight landmarks. Tests were performed with three of the landmarks removed. Anderson found that bees did not search at the position of best retinal match with the remaining landmarks, but instead moved further inside the semi-circle. He suggests that the bees attempt not to restore the retinal positions of landmarks but some measure of surroundedness. Another instance of the parameter hypothesis is the average landmark vector (ALV) model [58] which will be reviewed in section 1.3. Möller [73] discusses some key experimental results that models based on the parameter hypothesis can replicate which models based on the template hypothesis cannot replicate.

Aside from approaches based on the ALV model, all known biologically inspired approaches to robot visual homing are based on the template hypothesis. The work in this thesis continues in that tradition.

\subsubsection{The Snapshot Model}

Cartwright and Collett [6] snapshot model has been highly-influential, both in the 
study of insect navigation and in insect-inspired robot navigation. As such, a detailed review of this classic paper is provided.

The snapshot model was developed from experiments on honeybees. Honeybees were trained to enter a specially prepared room through a window situated a few metres from the hive outside. The bees were culled so that only one young fast bee was used at a time. The bee was trained to a food source inside the room to which it would return every five or ten minutes. Visual cues in the room were eliminated as much as possible and the food source was periodically moved such that the only visual information that the bee should be alble to employ would be an array of distinct artificial landmarks that surrounded the food source. The food source consisted of a small $(1 \mathrm{~cm})$ plastic cup filled with sucrose. The landmarks themselves consisted of matte black cylinders of around $40 \mathrm{~cm}$ in height and $4 \mathrm{~cm}$ in width. Modifications to the landmark array and the bees subsequent change in search pattern formed the independent and dependent variables of the experiment.

Cartwright and Collett found that honeybee learned the retinal size and bearings of landmarks as viewed from the location of the food source. If the food source was removed and the landmark array modified, the bees would concentrate their search at that point in space where the bearings and apparent sizes of landmarks were most similar to the snapshot image. Cartwright and Collett conducted a number of specific experiments, briefly reviewed below.

\section{Apparent Size}

Bees could be trained to a single landmark, and in tests with the feeder removed they searched at the position where both the landmark's bearing and apparent size corresponded best with the bearing and apparent size as seen from the feeder. In further tests with the size of the landmark reduced or enlarged, the bees would still search at a position where the apparent size matched. This would bring them closer to a smaller landmark, and further away from a larger landmark. Apparent size is not the only cue that bees could employ to situate themselves at a particular clistance from a trained landmark. Motion parallax and looming (rate of apparent size change) can also be used as distance cues. Apparent size, however, was found to match the experimental data most closely. 


\section{Edges}

Bees trained with a solidly coloured rectangular landmark exhibited a similar spatial distribution of search when confronted with a rectangular landmark that was, in effect, only the frame of the original landmark. This was taken to indicate that edges are the important visual features to which bees attend. The solid regions present in training did not need to be present during a later trial for the same search pattern to be observed. However, while the search distribution was similar, the bees displayed less tendency to actually initiate search with the framed landmark.

\section{Horizontal and Vertical Extents}

Experiments which independently altered both the horizontal and vertical extents of trained landmarks revealed that both of these aspects are attended to by homing bees. Changing one dimension of a landmark's size did not have as much effect as changing both.

\section{Landmark Bearings}

When trained with an array of three landmarks surrounding the food source the bees seemed to place primary importance on restoring the bearings of those landmarks. In tests with the sizes of some or all of the landmarks altered the search distribution remained largely unchanged. When given a choice between homing to a position where the bearings are correct and the distances incorrect or vice versa, the bees strongly preferred the position where the bearings are correct. Apparent size was not ignored but simply overrided by the bearing criterion.

\section{Orientation}

Experiments were performed to test the ability of bees to find the trained position after rotating the landmark array: When the array was rotated by $30^{\circ}$ bees searched in the position where the angle between landmarks matched the training situation. However, if the array was rotated by $90^{\circ}$ then the search distribution changed dramatically: Also, if the landmark array was rotated beyond $45^{\circ}$ during training the bees appeared unable to learn the feeder's location. This suggests that landmarks are stored with respect to external compass bearings. 


\section{The Five Models}

Cartwright and Collett developed five computer models intended to mimic the observed features of the honeybees' search patterns. These models were tested in a simulated two-dimensional world consisting only of free space and circular black landmarks. Within this world an agent perceives one-dimensional panoramic binary images. The models all process some representation of the snapshot and current images and generate a home vector. All five models employ the bearings of landmarks, matched between the snapshot and current images. The models differ in three ways:

Features: edges or regions. An edge can be described by its position and polarity: Regions are delimited by edges and are either light or dark. For both of these features a pairing process will be applied that matches edges to edges by comparing position and polarity, or regions to regions by comparing position and value.

Compass: A compass may or may not be used to restore the agent's orientation to that held at the snapshot position.

Correcting vectors: tangential and/or radial vectors. To describe the difference between tangential and radial vectors it is first necessary to describe how information can be extracted from the pairing of features.

Each pairing allows the extraction of vectors directed toward correcting that pairing. To correct a pairing means to move the agent such that the feature in the current. image is brought closer to the feature in the snapshot image. To pair regions, the closest matching region in the current image is found for each region in the snapshot. image. Closeness is defined by taking the angular distance between region centres. The paired regions must match (i.e. both light or both dark). Edges can be paired in a similar fashion. Two different vectors are generated for each feature pair. A radial vector is directed towards or away from the centre of the feature in the snapshot image. This vector is directed towards the feature if it has grown smaller in the current image and $180^{\circ}$ away if it has grown larger (radial vectors are not available if edges are used as features because an edge has zero width). A tangential vector is directed tangentially to the left or the right to match the direction of movement of the feature from the snapshot image to the current image. 
Various arrangements of landmarks were presented to the simulated bees. Search density profiles were generated and compared with results from actual bees on similar landmark arrays. Results on the fifth model most closely matched the experimental data. This model pairs regions as image features; uses a compass to compensate for changes in orientation; and combines both tangential and radial vectors to correct each pairing. A home vector is obtained by summing together all radial and tangential vectors. We will henceforth refer to this model as the snapshot model. An illustration depicting the model appears in figure 1.1.

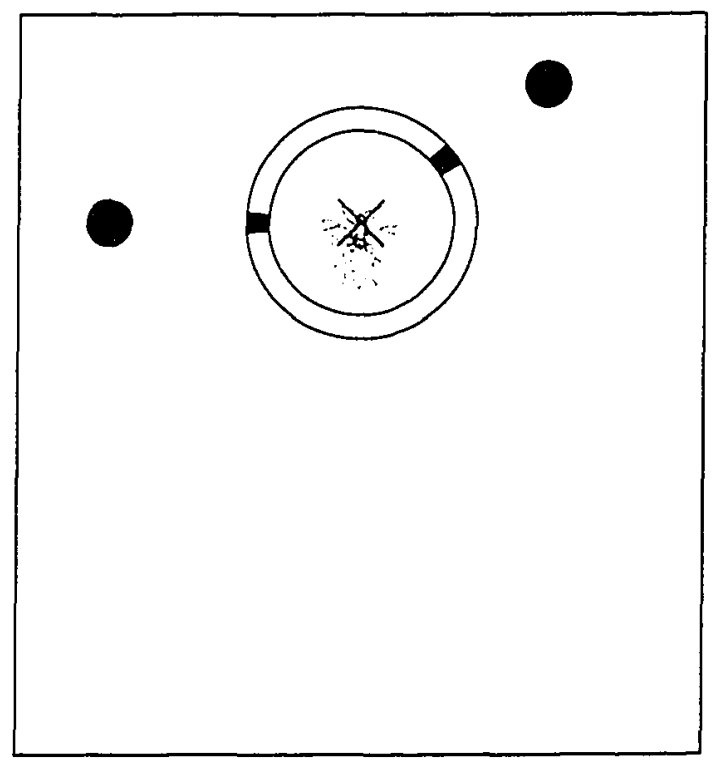

(a) At the goal

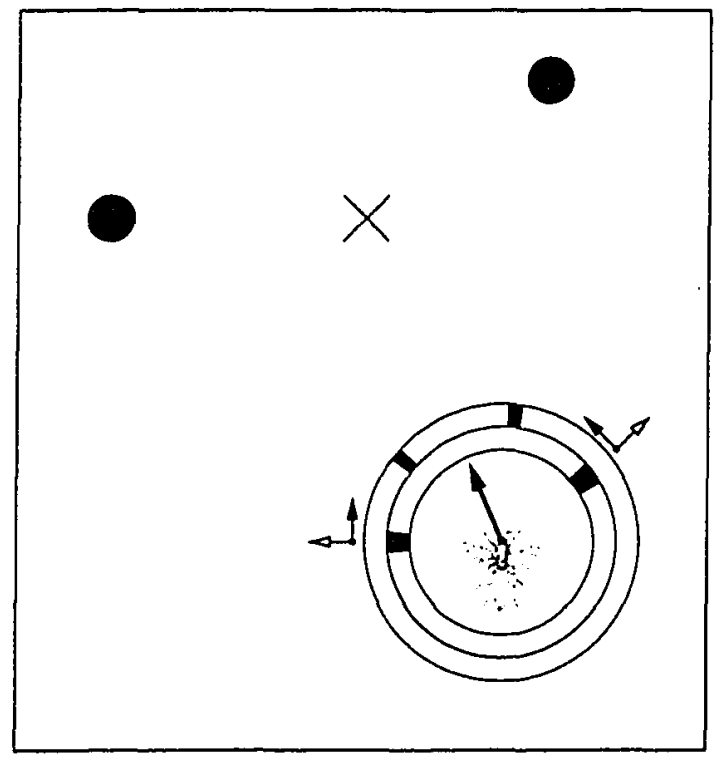

(b) Homing

Figure 1.1: The snapshot model. (a) The bee is at the goal and stores a snapshot image of the two landmarks (black circles). The annulus around the bee represents the snapshot image. (b) The bee has been displaced from the goal. The outer annulus represents the current image while the imner annulus is the snapshot image. Tangential vectors are shown with filled heads while radial vectors have open heads. The large vector centred on the bee is the home vector, computed by summing all tangential and radial vectors. For simplicity, pairings between non-landmark regions have not been included. 


\subsection{Robot Visual Homing}

Two major approaches to visual homing in robots have been proposed. These are known as associate homing and local homing [44]. Associative homing operates by sampling the environment at a number of positions and recording the viewed image and coordinates of each position. Homing is then achieved by recognizing the current location with respect to the stored image database. Local homing samples the enviromment only at the goal position and computes a home vector from the disparity between the representation stored at the goal and the representation of the current situation. For approaches based on the template hypothesis-often implicitly-this representation will be an image.

\subsubsection{Associative Homing}

The prototypical example of associative homing is Nelson's 'Tinytown' [81]. Nelson used a camera mounted on a robot arm that could sweep in a plane above a miniature town called Tinytown. A database of 120 images was captured from the downward facing camera. Each image was associated with a home vector directed towards the goal. Images were divided up into a $5 \times 5$ grid and the predominant edge direction in each cell was recorded. Thus, significant savings in storing the image database were obtained. Homing is achieved by processing the image from the camera's current location in the same manner and finding the best match with the image database. The method was successful for the majority of positions within Tinytown.

Jogan and Leonardis [51] used panoramic images for associative homing within an unmodified office environment. In this case the storage requirements are reduced by an eigenspace approach that records only those eigenimages which have the largest eigenvalues. This not only reduces the storage space from what would be required for the raw images, but also increases the accuracy and robustness of recognition. Good localisation results were obtained using this method which was also shown to be robust to occlusion.

Nevertheless, associative homing can incur a large computational cost and requires the homing agent to spend significant time capturing its image database ${ }^{3}$. Also, there

\footnotetext{
${ }^{3}$ To collect 170 images for the image database described in chapter 3 required approximately one hour. This cloes not include the time required to prepare the room (e.g. by placing tape markers for positioning and orientation). Also, significant manual assistance was necessary to position and
} 
is no evidence that insects or other animals densely sample their enviromments as would be required by an associative homing approach.

\subsubsection{Local Homing}

\section{Hong et al. [43]}

The system described by Hong et al. [43] appears to be one of the first robotic implementations of local homing. A camera pointed upwards at a spherical mirror is used to capture a one-dimensional image along the horizon. Characteristic points are computed as well-separated points in the image with a high estimated first derivative. Matches between characteristic points in the snapshot image and pixels in the current image are scored by the correlation of the surrounding windows. The method used by Hong et al. to compute a home vector from these correspondences between characteristic points is reviewed and tested in chapter 5 . The algorithm requires that the robot maintain a consistent orientation. To support this requirement, the robot platform is designed to move without rotation. To test the algorithm the robot is positioned along a sequence of 17 waypoints, spaced at one foot intervals in an office environment, and allowed to capture an image at each waypoint. It then homes to each waypoint in sequence, thus tracing out a route. The robot was found to be capable of fully retracing this route.

\section{Röfer [89]}

The wheelchair robot described by Röfer [89] operates similarly to that of Hong et al.'s, with a number of key differences. This approach retains three colour channels in the image and uses this colour information to create a correlation matrix between pixels in the snapshot and current images. An iterative search process is applied to find correspondences between pixels which have a high value in the correlation matrix, while also preserving the global ordering of image features. An estimation technique is used to detect changes in orientation of the robot between the snapshot and current images. Röfer's experimental approach is also similar to that of Hong et al.. Particular routes through an office environment are learned by recording a orient the capturing robot. 
sequence of snapshots. The robot was able to retrace these routes with a reasonable degree of accuracy.

\section{Franz et al. [26]}

Franz et al. [26] present a very different approach to homing that is based on an image warping model as opposed to the search for correspondences. A range of possible distances, directions, and orientations in relation to the goal is searched. For each triple of distance, direction, and orientation the current image is warped and then compared with the snapshot image. The best matching set of parameters is used to determine the direction of the goal. A robotic implementation of this method achieved reasonably good results on a table-top with toy houses comprising the landmarks. Some further results on this method by Weber et al. and later by Möller will be discussed below. The warping method has the advantage of solid mathematical underpinnings, good performance, and the ability to estimate orientation along with homing direction. In terms of its biological plausibility; Franz et al. believe that the warping method could be simply implemented in neural circuitry with matched filters for all possible displacement fields. Möller argues, however, that the number of neurons required for such a scheme would be far in excess of the number of neurons in the brains of most insects. This method is thoroughly reviewed in chapter 4 where it is shown to exhibit superior performance to two other homing methods (reviewed below). It is subsequently used throughout this thesis for comparison with the methods developed here.

\section{Weber et al. [104]}

Weber et al. [104] present and evaluate a number of variations on the algorithm of Hong et al.. The primary focus is on using different algorithms to search for the best landmark correspondences. These different correspondence algorithms range from exhaustive search to the simple closest-match pairing employed in the snapshot model. It is found that the expensive and elaborate algorithms which generate the most correct correspondences are unnecessary for successful homing. Approximate correspondences are generally sufficient. Weber et al. explain this in the following way: When the agent is far from the goal inaccuracies in the home vector caused by incorrect correspondences are not critical. Yet when close to the goal, where accuracy 
is more important, correct correspondences are more easily achieved. This result has been inferred from experiments on a large set of randomized simulation enviromments. Successful homing is almost always achieved in these environments, therefore Weber et al. focus on optimizing small differences between their methods. Weber et al. also do experiments in a more challenging visually-bounded world and on a mobile robot.

Another interesting result from this paper concerns a comparison between their method and the warping method of Franz et al.. In their visually-unbounded simulation environments, where success of their method is almost universally achieved, it is found that the warping method fails for many cases. Generally, the warping method fails when homing begins from a position outside the immediate vicinity of the landmarks visible from the goal. These landmarks roughly define a wall outside of which the warping method fails.

\section{Stürzl and Mallot [95]}

Stürzl and Mallot [95] presents a variant of the warping method that operates not on a typical image of light intensities, but on a range image. This image is arrived at through a special purpose panoramic stereo mirror and encodes the approximate distance of viewed objects. The method is tested on images from a robot in an artificial arena populated by toy houses. The original warping method is found to exhibit better performance in this environment but its performance breaks down under a change in illumination. The variant using the special stereo mirror is more robust against changes in illumination. The effective range for this method is constrained to about $1 \mathrm{~m}$ by its small stereo baseline. As mentioned above, the use of stereo vision for insect navigation appears unlikely. Nevertheless, rodents and other animals may well make use of stereo for navigation due to the larger distance between their eves.

\section{Lehrer and Bianco [62]}

As mentioned in section 1.2, bees, wasps, and ants perform special maneuvers known as learning flights or walks. These behaviours may allow these insects to determine the distance of landmarks to the goal and perhaps to select nearby reliable landmarks to use for homing. Lehrer and Bianco [62] summarize some of this evidence and show that a similar behaviour can enable a robot to select reliable landmarks for homing. 
Lehrer and Bianco found that incorporating a biologically inspired 'turn-back-andlook' behaviour to select distinctive landmarks increased the efficacy of a robot homing within an unmodified office environment.

\section{Gourichon et al. [29]}

The approach of Gourichon et al. [29] is similar to that of Röfer's in that it uses colour information for matching. It is also similar to Weber et al. in that it employs a more elaborate correspondence process. In this case a dynamic programming approach is used to match sectors in the snapshot and current images. Two-dimensional regions of like colour are used as the sectors to match. This appears to be the first attempt at local homing that employs two-dimensional image features. The vertical position of the sector in the image is used as part of the matching process but is not used to glean extra information about how to correct that pairing. By contrast, all of the methods developed in this thesis use the vertical position of features for both of these purposes. Gourichon et al.'s robot, unlike all of the other approaches described here, does not capture full omnidirectional images, but only partial panoramas covering $200^{\circ}$. A robotic experiment in an office environment shows moderate success.

\section{Lambrinos et al. [58]}

Lambrinos et al. [58] describe a range of biologically-inspired robotic experiments. A detailed model of path integration in Cataglyphis is developed and evaluated along with several variants of the snapshot model. The first variant is called the proportional vector model. It operates identically to the snapshot model except that the tangential and radial vectors are each weighted by the amount of difference in region position and size (respectively) between the snapshot and current images. Another variant is the difference vector model which lacks radial vectors and replaces the tangential vectors by secant vectors. Each secant vector is calculated by taking the difference of the vector pointing to a region in the snapshot image with the vector pointing to the matching region in the current image. The behaviour of the difference vector model is thought to be qualitatively similar to the proportional vector model. This model is introduced only because it is mathematically tractable for comparison with the Average Landmark Vector (ALV) model.

The ALV model extracts landmarks from a one-dimensional image in the same 
manner as the snapshot model. For each landmark a unit vector is created to point to the centre of that landmark. These unit vectors are averaged together to form the Average Landmark Vector (ALV) for that image. $\mathbf{A L V}_{\mathbf{s}}$ is found at the snapshot location and, while homing, $\mathbf{A L V}_{\mathbf{c}}$ is found for the current image. Surprisingly enough, all that is required to calculate an estimated home direction is to subtract these two vectors. The vector $\mathbf{A L V} \mathbf{c}-\mathbf{A L V}_{\mathbf{s}}$ estimates the home direction. Figure 1.2 illustrates the ALV model. This model requires that the snapshot and current images be taken from the same orientation. The ALV model is quite parsimonious in that it requires only a single vector to be stored in place of the snapshot image, and also because it calculates the home direction so easily without any expensive correspondence process or image warping.

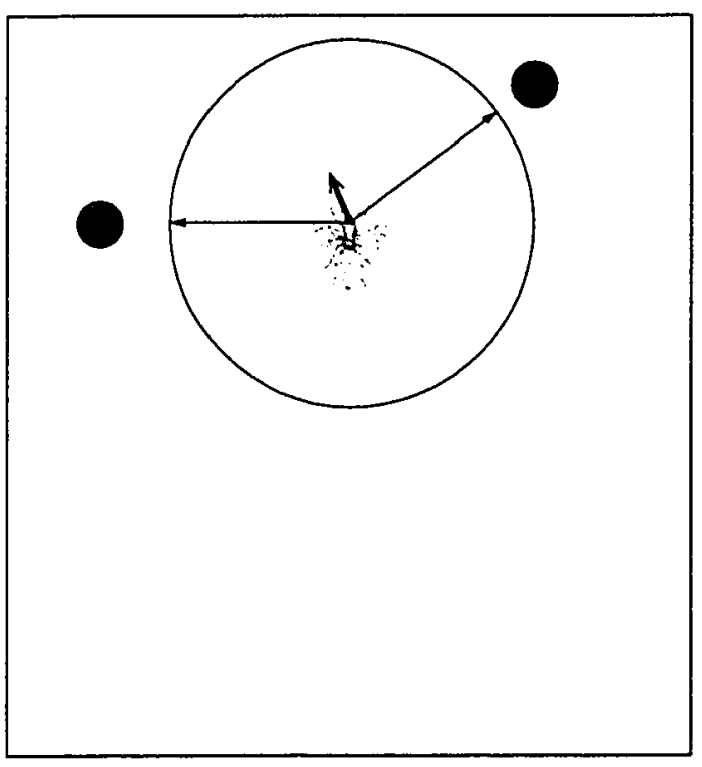

(a) At the goal

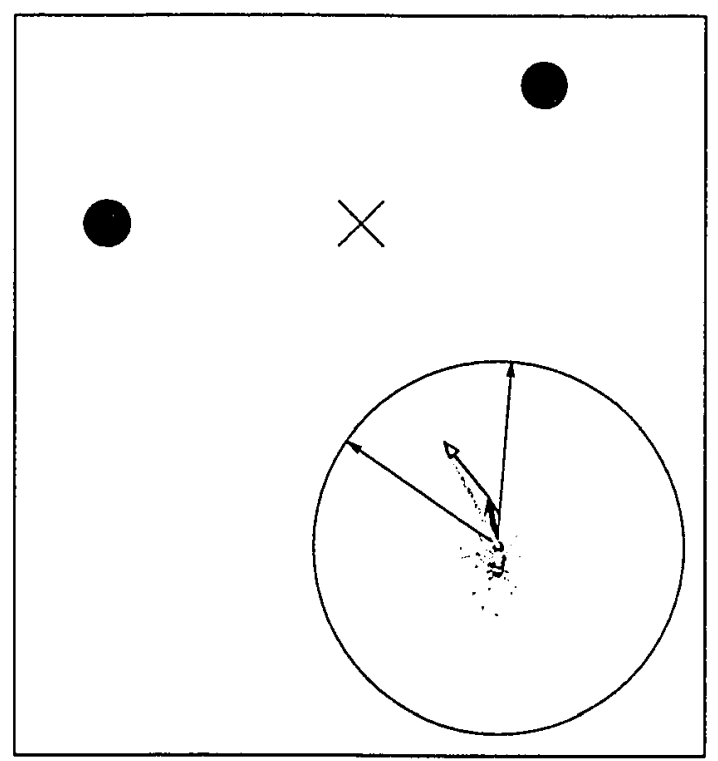

(b) Homing

Figure 1.2: The ALV model. (a) The bee is at the goal. Landmark vectors (thin shafts) are averaged together to produce the average landmark vector for the snapshot position, $\mathbf{A L V}_{\mathbf{s}}$ (thick shaft). (b) The bee has been displaced from the goal. Landmark vectors are averaged to produce the average landmark vector for the current position $\mathrm{ALV}_{\mathbf{c}}$, (grey thick shaft). The home vector is found through subtraction $\mathbf{A L V}_{\mathbf{c}}-\mathbf{A L V}_{\mathbf{s}}$ (arrow with open head). The circles surrounding the bee indicate that the landmark vectors are unit vectors. 
Lambrinos et al. showed that the ALV model generates home vectors identical to the difference vector model for cases where the landmarks were correctly paired between current and snapshot images. Further, they suggest that the ALV model always establishes the correct pairing implicitly.

Experiments in simulation demonstrate the homing performance of the snapshot, proportional vector, and ALV models in a simulated environment. This environment is two-dimensional and filled with 27 circular landmarks/obstacles of varying size. One characteristic of this simulation environment, which is shared by the environment used to develop the snapshot model, is that it consists of a cluster of landmarks in the centre of the environment with nothing else perceptible outsicle this cluster. Occlusions can prevent landmarks from being perceived but the simulated agent's range of vision is infinite. Thus, these simulation enviromments are distractor-free and offer no possibility for landmarks to go out of range or become indistinct. A robotic experiment is also provided which provides a real-world demonstration of the performance of the proportional vector model. The robotic test environment is a flat expanse of desert terrain with artificial landmarks consisting of three large black cylinders. The thorny problem of consistently detecting visual landmarks is rendered trivial in this simplified environment. It is quite interesting to note, however, that this test environment is the same one in which a number of experiments on Cataglyphis have been performed.

\section{Möller [72]}

Möller [72] implements the ALV model in a robot with purely analog hardware and also provides support for this model both theoretically and in terms of biological plausibility. Global convergence of the ALV model is shown, under the assumption that the same set of landmarks remains visible; that there are at least two landmarks; and that not all landmarks are on a line through the goal. A number of other properties of the ALV model are discussed, including the impact of changes in the number of landmarks. The robot built to implement the ALV model consists only of analog components. Möller argues that analog components inherently share a number of important properties with biological systems, such as the lack of a global clock, paralellism and, continuous-value signals. This model is designed to operate in a highly simplified real-world setting that consists only of distinct light and clark 
sectors. Experimental results show good performance but it is not clear whether the same system could be modified for application in a more natural setting (see below). A final experiment shows that this analog implementation of the ALV model is able to roughly replicate some of the search patterns of bees. Finally, Möller argues that given the simplicity of his analog implementation a similar mechanism might well exist in the brains of insects.

\section{Möller et al. [75]}

Möller et al. [75] apply the ALV model to the problem of homing in an office environment. The basic ALV model originally described by Lambrinos et al. remains unchanged, but requires an extra pre-processing stage to extract the features used to compute the ALV. One-dimensional images are drawn from two-dimensional images of a conical mirror mounted atop a robot. Features in these one-dimensional image are extracted using an adaptive thresholding scheme. The method was found to work reasonably well within the test environment.

This same method was compared with the warping method by Möller [74]. The warping method was found to be superior both in terms of homing performance and in terms of resilience to changing parameter settings. Performance of an earlier variant of the method CGSM, described in chapter 6, was also found to yield superior performance [99].

\section{Möller et al. [76]}

The inspiration for CGSM comes from Möller et al.'s [76, 77$]$ neural snapshot model, which implements the snapshot model using a biologically plausible neural architecture. For reasons of simplicity; the neural model does not replicate all aspects of the snapshot model. For example, it employs proportional vectors (as in the proportional vector model) and utilizes edges as features. The neural model is composed of lavers of processing rings where the neurons in each ring interconnect locally-except for the final inside ring which has more convergent connections. The outermost ring is exposed to a one-dimensional panoramic image of the homing agent's environment. One of the most interesting aspects of the neural snapshot model is its method for matching edges between the snapshot and current images. This matching is achieved by first growing a potential surface and then using the gradient of this surface to 
determine the direction of correspondence. Here the potential surface is a one-dimensional image which is peaked at the positions of features in the current image, and decays according to the distance from these positions. The neural snapshot model actually employs two potential surfaces, one decaying to the right and one to the left. Each potential surface neuron passes on a degraded signal in one direction, either clockwise or counterclockwise, around the toroidal image. Sensing both surfaces at a single position allows a cell to determine whether the nearest feature of the current image is to the left or to the right.

Figure 1.3 depicts the operation of this model. The bottom laver in figure 1.3(b) indicates the distance and direction of matching for the four edges extracted from the snapshot image. For example, the left-most edge in the snapshot image is paired to the left with an edge from the current image. The pairing is made to the left because the potential surface indicating a left-ward pairing (dashed line) is higher at the position of the snapshot edge than the solid line indicating a right-ward pairing.

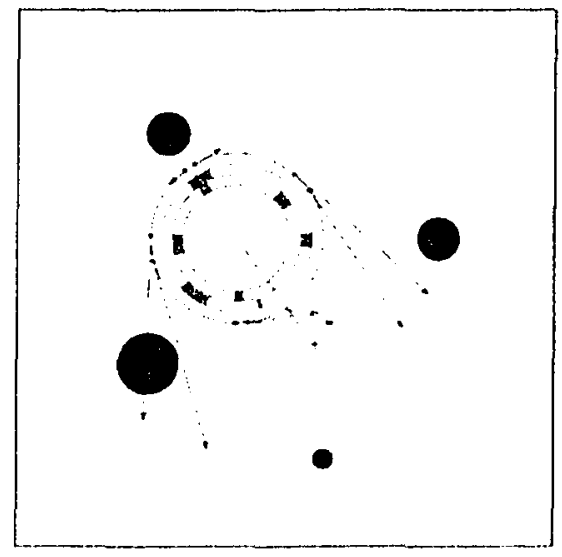

(a)

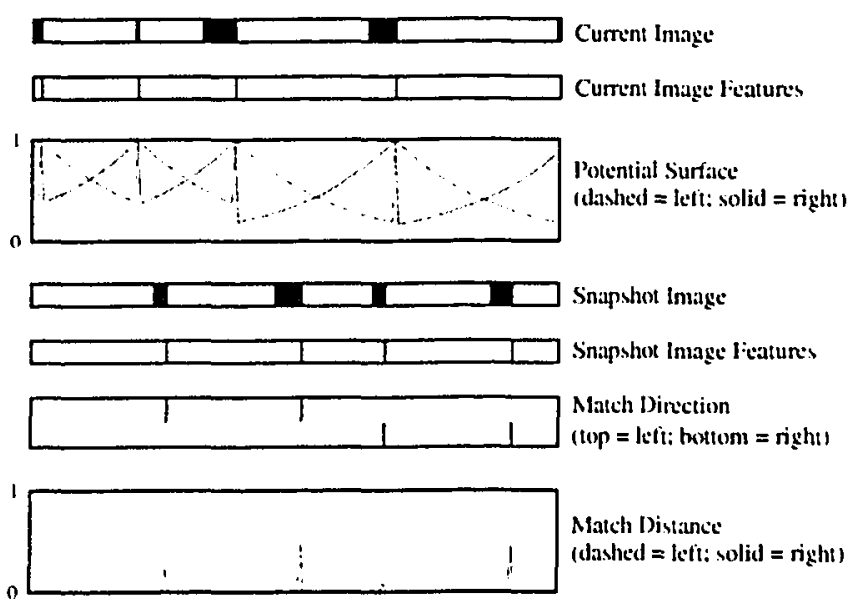

(b)

Figure 1.3: The neural snapshot model. (a) Black circles indicate landmarks. Current and snapshot views are as described in figure 1.1. Correspondence vectors are shown attached to a ring around the agent. The home vector is shown at the center of the concentric rings and is directed approximately toward the goal ('t' symbol). (b) Activation in the neural circuit for the situation depicted in (a). Note that only the processing for the right edges of image sectors is shown. Adapted from [it]. 
Möller et al. also describe how vector contributions are calculated from matches between edges using the same neural architecture. The performance of the neural model is found to be roughly comparable with the original snapshot model within a similar simulated world. The neural model shows that the snapshot model can be instantiated using the biologically-plausible architecture of simple locally-connected units arranged in retinotopic layers. Due to the simplistic feature detection apparatus, the neural model is not directly applicable to real-world environments (although it would likely operate well in the simplified environment that the analog ALV model was tested in).

\section{Hafner and Möller [35]}

Hafner and Möller [35] describes neural networks which learn a visual homing algorithm. Pairs of one-dimensional images are fed as input into a network which is trained to compute home vectors from the input images. The first image of the pair is the snapshot image, while the second is the current image. A number of different network architectures and learning rules are attempted and it is found that the same basic mechanism is converged to in all cases. This mechanism is found to be a variant of the ALV model, but one which does not require feature extraction. Instead, this mechanism extracts the image's centre-of-mass. In subsequent work the same method was found applicable to robot homing in an office environment [33]. This method is described in detail in chapter 4 .

\section{Zeil et al. [108]}

A markedly different approach to homing is attempted by Zeil et al. [108]. Zeil et al. capture images of an outdoor enviromment using a mobile robotic gantry. They find that the root mean square (RMS) difference between images varies smoothly for images taken at different points in space. Further, they found that the difference function between two images increases mostly monotonically with distance. Thus, they propose a homing method based on gradient clescent on this distance function. The RMS difference between the current image and the snapshot image is found. The agent then takes one or two exploratory steps and computes the RMS difference for these other positions in space. A rough approximation of the local gradient of the difference function can thus be computed. Moving in the direction of the negative of 
this gradient then guides the agent towards home. The computational expense for this strategy is quite low, and the fact that Zeil et al. demonstrate that it works on outdoor images makes it appear quite biologically plausible. This work will be reviewed in detail in chapter 4 .

\subsubsection{Discussion}

Clearly a wide range of approaches to robot visual homing have been proposed. There are several dimensions of differences between these approaches. Two of these dimensions - template vs. parameter hypothesis, and associative vs local hominghave already been described. Considering only the local homing approaches reviewed above, only those approaches which relate themselves to the ALV model adhere to the parameter hypothesis. This leaves us with quite a number of template-based local homing approaches undistinguished from each other.

We can pick these apart by considering the complexity of these methods. Most of these approaches employ a search process. The search for Hong et al.'s method aims to find the best matching window in the current image for the window surrounding each characteristic point in the snapshot image. The search processes for Röfer, Weber et al., Lehrer and Bianco, and Gourichon et al. are all quite similar. Franz et al. use a search through parameter space to find the parameters generating the warped image most similar to the snapshot image. Stürzl and Mallot utilize the same underlying method. However, the methods of Möller et al. [76], Hafner, and Zeil et al. involve no such search process. The only iterative procedure applied by any of these methods is the growth of the potential surface by Möller et al.'s [76] method. The methods based on the ALV model all share the lack of a search process. Thus we can introduce the classification dimension: search vs. no search.

The breakdown of all local homing methods reviewed above according to these dimensions is provided in table 1.1. The right-most column in the table comments on how each method was evaluated. The column labelled 'R.P.' will be discussed in section 1.4.2. 


\begin{tabular}{|c|c|c|c|c|}
\hline Reference & $\begin{array}{c}\text { Parameter } \\
\text { vs. Template }\end{array}$ & Search & $\overline{R . P .}$ & Evaluation \\
\hline Hong et al. [43] & Template & Yes & No & $\begin{array}{l}\text { Route through office } \\
\text { env. }\end{array}$ \\
\hline Röfer [89] & Template & Yes & No & $\begin{array}{l}\text { Route through office } \\
\text { env. }\end{array}$ \\
\hline Franz et al. [26] & Template & Yes & No & Toy house arena \\
\hline Weber et al. [104] & Template & Yes & No & $\begin{array}{l}\text { 2-D sim. and office } \\
\text { env. }\end{array}$ \\
\hline Stürzl and Mallot [95] & Template & Yes & No & Toy house arena \\
\hline Lehrer and Bianco $[62]$ & Template & Yes & No & Homing in office env. \\
\hline Gourichon et al. [29] & Template & Yes & No & Homing in office env. \\
\hline Lambrinos et al. [58] & Both $\dagger$ & Yes/No & No/Yes & $\begin{array}{l}\text { 2-D sim. and desert } \\
\text { env. }\end{array}$ \\
\hline Möller [72] & Parameter & No & Yes & Simplified arena \\
\hline Möller et al. [75] & Parameter & No & No & Homing in office env. \\
\hline Möller et al. $[\bar{i} 6]$ & Template & No & Yes & $2-\mathrm{D} \sin$ \\
\hline Hafner [3:] & Parameter & No & Yes & Homing in office env. \\
\hline Zeil et al. [108] & Template & No & Yes & $\begin{array}{l}\text { Homing in outcloor } \\
\text { env. }\end{array}$ \\
\hline
\end{tabular}

Table 1.1: Reviewed approaches to local homing. † Lambrinos et al. [58] describe template-based variants of the snapshot model, as well as the parameter-based ALV model. 


\subsection{Criteria}

The aim of this thesis is to develop methods for visual homing which perform well, exhibit low computational complexity, and are biologically plausible.

From the technical perspective, it is perfectly understandable to posit such criteria as performance and complexity. Yet the additional criterion of biological plausibility may have technical benefits as well. After all, animals have a demonstrated ability to navigate in unstructured environments. No existing artificial methods can exhibit comparable performance. Further, information processing in animals is fast, flexible, and consumes very little power. More specifically, the approach we take towards biological plausibility is adherence to the constraint of retinotopic processing. A retinotopic processing matrix is an inherently parallel processing system and is therefore attractive from the complexity standpoint.

Yet what are the value of biological plausible algorithms to the biological community? As described below, while biological plausibility is assessed qualitatively; it does exist on a continuum. If effective solutions to a problem are limited, and only a few effective solutions are identified as biologically plausible, then biologists can look for traces of these solutions in both the animal's behaviour and in its brain.

Performance will be our primary criterion. If a homing method is not effective then it will be of little use from either the technical or biological perspectives. The means by which performance is measured will be described in detail in chapter 3 .

Biological plausibility and computational complexity are secondary criteria. There are a number of links between these two criteria which compels us to consider them together. For example, some methods may require a level of computation which the brain of an insect cannot support. The next section will focus on biological plausibility. and the particular approach taken towards it in this thesis.

\subsubsection{Biological Plausibility}

Many of the specific behaviours used by insects to navigate have been elucidated but the mechanisms underpinning these behaviours remain unclear. Meanwhile, one cannot implement any behaviour in a robot without specifying the mechanisms to achieve it. If a robot and an insect are both performing the same behaviour under similar conditions then it is possible that, through the engineer's design process, the robot 
and the insect are employing the same fundamental mechanisms. This is unlikely to occur by chance. Yet if there are a range of levels of effectiveness of mechanisms, and we assume that both robot and insect are using highly effective mechanisms, then it is more likely that the robot and insect are using the same mechanisms. Further, if the human engineer decided to implement the robot's mechanisms using similar constraints to those the insect operates under, then it will be more likely again that the robot and insect are using the same mechanisms. We define biological plausibility as the likelihood that the same mechanism is being used in both insect and robot.

For the case of visual homing, the assumption that insects employ highly effective mechanisms seems logical. When an insect is homing to its nest, the lack of ability to find the nest may well be fatal. If an insect is equipped with an inefficient yet reliable homing method it may survive. By inefficient, we mean that the trajectory followed by the insect will deviate strongly from the optimal straight line. Yet there would exist strong evolutionary pressure for insects to adopt optimal strategies. This pressure is particularly apparent when one considers foraging. If visual homing is employed throughout a foraging trip, then the amount of material returned per unit time will be proportional to the efficiency of homing. This being said, it is important to note that these are merely suppositions. The author knows of no direct study on the efficiency of visual homing in insects.

The other component of biological plausibility is that the robot's mechanisms adhere to similar constraints. The next section discusses one possible structural constraint.

\subsubsection{Retinotopic Processing}

According to Möller "...nothing is known so far about the neural circuits that realize visual homing in insect brains" [72]. This statement was recently re-affirmed by T.S. Collett (personal communication, 2003). What kind of constraints can be developed if we have no information about these neural circuits? The answer is to step back and look at the overall principles that seem to govern the processing of visual information in insects and other animals.

The Nobel prize winning work of Hubel and Wiesel [47] probed early visual processing in the cat visual cortex. They describe various cells in the cat visual cortex as exhibiting different receptive ficlds. According to Hubel and Wiesel, "the receptive 
field of a cell in the visual system may be defined as the region of retina (or visual field) over which one can influence the firing of that cell." They found that cells sharing receptive fields were organized into columns with the retina on top and increasingly elaborate, yet overlapping, receptive fields found as one descends. This structure is retinotopic: "each level of the system is organized like a map of the retina." [84].

It is also clear that the neural processing of visual information in insects is retinotopic. In clescribing motion processing within the framework of the fly's visual system Egelhaaf et al. state "the visual system is organized in a retinotopic way by columnar elements" [18]. They describe how patches of local visual motion are detected by lower-layer neurons which converge to higher Tangential Cells (TCs). These TCs integrate local motion from many lower-level cells, thus exhibiting very wide receptive fields. The lower level cells that compute local motion do so through lateral interactions between neighbouring cells on the same level within adjacent columns. The neural circuit that exists between these cells is known as an Elementary Motion Detector (EMD) [17]. The response characteristic of certain TCs appears to match the optical flow fields generated when the insect is rotated about one of its principal axes [55]. The point we wish to emphasize here is not the particular details of the fly's motion detection system, but the fact that this system's neural architecture is organized retinotopically:

Retinotopic order is found also throughout the optic lobe of the bee. A bee's optic lobe consists of three separate neural masses called neuropils. These three neuropils are the lamina, medulla, and the lobula. A retinotopic arrangement of cells can be observed within these three neuropils. A schematic in figure 1.4(a) shows the overall layout of the optic lobe. Figure 1.4(b) highlights the retinotopic organization of the optic lobe-the clear visual impression is of parallel fibres projecting inwards.

The neural fibres that travel from the lamina to the medulla, and from the medulla to the lobula, travel in a single retinotopic bundle. Interestingly; these retinotopic bundles include a twist which reverses the global ordering. Fibers from the posterior portion of the lamina project to the anterior portion of the medulla and vice versa (these twists are illustrated in figure 1.4(a)). Yet, the highly regular retinotopic arrangement of identical cartridges is maintained despite these two twists. At some point within the lobula retinotopic order appears to break down. This is known because fibres leave the lobula towards the protocerebrum in several bundles [86]. 


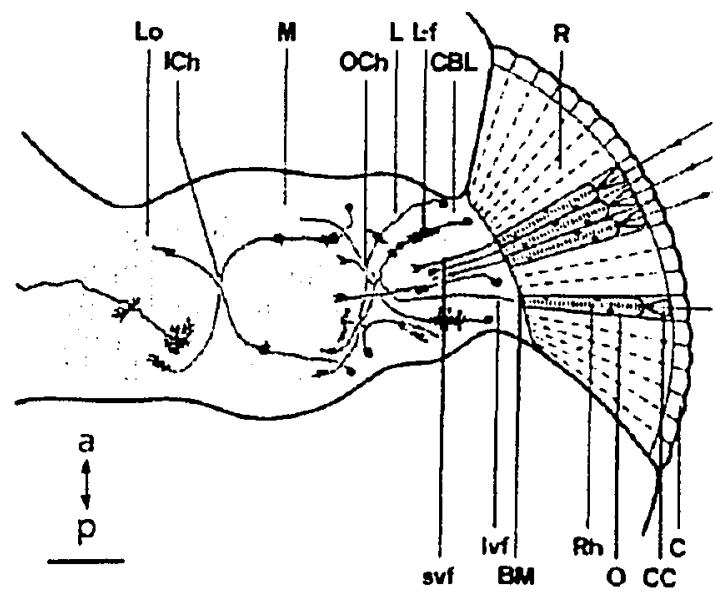

(a)

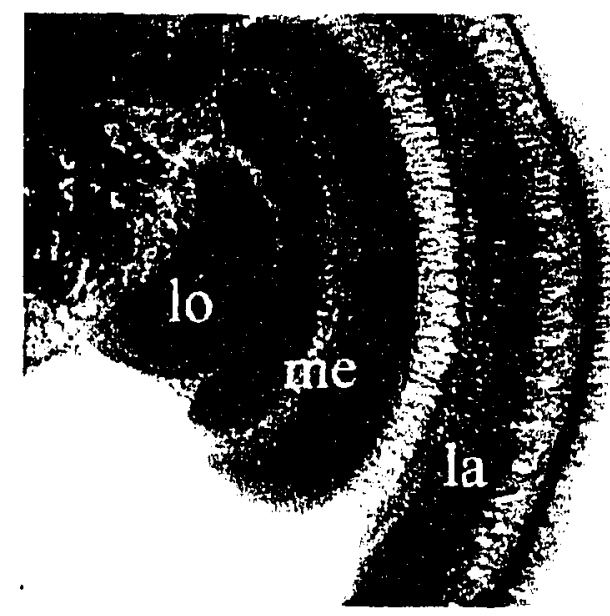

(b)

Figure 1.4: The optic lobe of the bee. (a) A schematic showing the three neuropils, the lamina $(L)$, medulla $(M)$, and lobula $(L o)$. On the right the retina $(R)$ is shown as well as the ommatidia $(\mathrm{O})$ or eye facets. Various neurons that project between neuropils are also shown. These neurons are repeated across the retinotopic mosaic. The axis shows anterior (a) to posterior (p) orientation. Taken from [32]. (b) Slide from the brain of a honeybee showing the retinotopic organization of the three neuropils, lamina (la), medulla (me), and lobula (lo). Adapted from [19].

These three neuropils comprise the optic lobe of the bee's brain. Of the total 850,000 neurons in the bee brain, approximately half of these are in the optic lobe [42]. Thus, it would be fair to say that nearly half of the bee's brain is devoted to the retinotopic processing of visual information. The caveat of "nearly half" is added because we do not know precisely where in the lobula does retinotopic order end.

It is clear that bee vision cannot be properly understood without reference to persistent retinotopic order. It is also clear that with the neural investment of half of its brain we can expect that the optic lobe should perform some significant tasks that camnot easily be offloaded to other parts of the brain.

\section{The Retinotopic Matrix}

A number of researchers who have built computational and/or robotic models inspired by insect visual systems have employed a retinotopic structure of processing elements. 
Franceschini et al. [21] built a robot inspired by components of the fly motion detection system mentioned above. The robot has an array of hardware-implemented EMD units arranged retinotopically in a ring. Its control system integrates the EMD outputs to steer the robot away from obstacles. A similar example, also inspired by the fly but applied to a more low-level robotic task is presented by Harrison [38]. Rind [87] presented a retinotopic neural model for the escape reflex of locusts. This model's network is tuned to provoke an escape response whenever an object approaches on a collision course. Huber et al. [48] implemented a robotic model of the fly's ability to stabilize its course and fixate on objects. Again, a retinotopic arrangement of EMD's was employed. In Huber et al.'s robot lower layers do spatial and temporal filtering of the input image before the layer of EMD's is applied. The local motion vectors generated by the EMD's is integrated to control the robot's steering mechanism. Additional examples of retinotopic neural models of insect vision include two which we have already discussed. These are Möller et al.'s neural snapshot model and the analog ALV model.

The input to all of these models is an image of the agent or robot's surroundings. Processing is applied to this image retinotopically until some stage is reached where behaviourally relevant information can be extracted. A wide-field integrating layer (or layers) is finally applied to extract this information. Typically, this final layer will transform the activity of the previous layer into a motion vector that will actually control the agent or robot.

Building upon this frameriork we define a retinotopic matrix as an abstraction of what is known about low-level visual processing in animals. A retinotopic matrix is composed of layers of simple neuron-like elements arranged retinotopically. These elements can receive input only from other elements within their immediate neighbourhoods, and from elements at the same position within previous layers. Depending upon the complexity of computation required from individual elements, a single element may actually be composed of a group of neurons that act cooperatively ${ }^{4}$. We now define a classification for various computational operations based upon the retinotopic matrix.

\footnotetext{
${ }^{4}$ This is why we use the term clement, as opposed to neuron.
} 
Retinotopic-Compatible We refer to any operation that can be implemented in a retinotopic matrix as retinotopic-compatible. Examples include:

- Convolution (e.g. low-pass filtering, finding spatial derivatives)

- Determining whether an image pixel is a local minimum or maximum with respect to neighbouring pixels ${ }^{5}$.

- Determining the local minimum or maximum value amongst neighbouring pixels $^{6}$

- Computing the value of any simple expression, which may include neighbouring values of the retinotopic matrix as inputs (e.g. $V_{i-1}(x-1, y)+\tan (x)^{2}$, where $(x, y)$ is the index of the current image position and $V_{i-1}(x-1, y)$ is the value of an adjacent neighbour in the previous layer)

Convergent Summing Operations Not all processing that occurs in the visual systems of insects is retinotopic. So-called wide-field neurons pool inputs from many neurons. We refer to an operation that can be implemented by pooling inputs from layers of a retinotopic matrix as a convergent summing operation. A convergent summing operation takes either a single row, a single column, or a whole laver and finds the sum.

Simple Global Operations Some computation must be allowed at the level of these wide-field neurons in order for the behaviour of the agent to be coordinated and coherent. Computations at this level are global, but if they are sufficiently simple then we label them simple global operations. An operation is sufficiently simple if the required amount of processing time is independent of such quantities as the size of the image, or the number of extracted features.

As an example consider implementing the ALV model. The computation of individual landmark vectors can be achieved by retinotopic-compatible operations (as

\footnotetext{
${ }^{5} \mathrm{~A}$ whole body of literature exists on winner-take-all neural networks which collectively determine which single neuron is the minimum or maximum (see chapter 14 of [36]).

${ }^{6}$ Operations such as determining the local minimum value would seem to require a search process. However, if the area searched is of small fixed size then the operation takes constant time. For instance, Hassoun and Sanghvi [40] propose a small neural network for determining the minimum value of five units.
} 
in the analog ALV model [72]). Summing these landmark vectors together to produce the average landmark vector is then a convergent summing operation. Finally, performing the subtraction $A L V_{c}-A_{L V}$ is a simple global operation.

Complex Global Operations Many methods simply do not fit within the framework clescribed above. These are methods which involve such operations as searching and sorting. Searching and sorting can be computed by a network of distributed neuron-like processors, but these processors would not have retinotopic connectivity: Computations at the global level which do not qualify as simple global operations will be termed complex global operations.

\section{Implementation}

A retinotopic matrix is an inherently parallel processing system. As such, it can either be simulated on a serial computer (as is done here) or implemented on a parallel computer or customized hardware platform. In fact, such an implementation must be imagined to allow a fair comparison between methods constrained to retinotopic processing and unconstrained methods. If simulating a retinotopic matrix with $m$ processing layers, each with resolution $u \times h$, the time complexity to complete processing of one image would be $O(m w h)$. However, if the implementation is parallel the time complexity is just $O(\mathrm{~m})$. This analysis assumes that the operations performed by processing elements all take constant time.

\section{Retinotopic Processing Constraints}

If a homing method consists only of retinotopic-compatible, convergent summing, and simple global operations then we say that this method satisfies the constraints for retinotopic processing.

As described above, the computational complexity of a method which satisfies these constraints will depend on the implementation medium. Further, it will not generally be the case that all retinotopic-compatible operations take constant time. The complexity of retinotopic-compatible operations is controlled by the size of local neighbourhoods and by the complexity of any functions which need to be evaluated. For these reasons a detailed accounting of complexity for methods satisfying the constraints of retinotopic processing will not be provided. 
Table 1.1 has a column labelled 'R.P.' which stands for retinotopic processing. All of the methods with 'Yes' in this column either explicitly constrain themselves to retinotopic processing (such as Möller et al. [76], Möller [72], or Hafner [33]), or else can easily be seen to fit within that framework (Zeil et al. [108]).

Methods which fail to meet the constraints of retinotopic processing are considered less biologically plausible. However, such methods cannot be ruled out unless it can be shown that they require more computational power than a typical insect brain. Claims of this kind will be enabled by a pair of very rough assumptions ${ }^{\top}$. The first assumption is that any low-level operation on a single image pixel requires one neuron. For example if we were to square all pixels in an image of size $w \times h$ then $w h$ neurons would be required. This will generally be a conservative assumption as it does not take the storage of products and intermediate products into account. The second assumption is that all operations occur in parallel. It is well known that parallel processing is ubiquitous in nature. Indeed, the prevalence of parallel processing in vision has been argued above-particularly for the case of insects. Nevertheless, this remains a strong assumption. The purpose of making these assumptions is to allow a complexity analysis to be performed which will determine the number of low-level operations required-and therefore-the number of neurons required. If this number is in excess of the approximately one million neurons in an insect's brain then we have a reasonable basis to state that the method in question is less biologically plausible than it might otherwise be.

Apart from any relationship to biological systems, the constraints of retinotopic processing restrict us to methods which are feasible for implementation in parallel hardware. A single layer of a retinotopic matrix could be mapped onto the logic blocks of an FPGA, with multiple layers represented by a stack of FPGA's with vertical interconnections. Another alternative would be a lavered VLSI implementation. Homing methods developed without any constraints on processing would have no guarantee of being feasible candidates for parallel implementation.

\footnotetext{
'Similar assumptions were made in Möller's [7.4] analysis of Franz et al.'s warping method.
} 


\subsection{Research Question}

Finally, we are in a position to state the research question for this thesis:

"Can the visual homing problem be solved by methods which are both competitive in performance, and yet adhere to the constraints of retinotopic processing?".

That methods should exhibit good performance is desirable, both from technical and biological perspectives. In the absence of perfect methods, however, performance is relative. Therefore we seek methods that exhibit competitive performance. The literature review on robot homing presented in section 1.3 uncovered few comparative studies between homing methods. Yet where comparisons were made between methods, Franz et al.'s warping method was always included in the comparison. This method seems to have emerged as the de facto standard in robot visual homing. Thus, it is used here for comparison. Any method that meets or exceeds its performance according to the performance metrics established in chapter 3 will be considered competitive.

The merits of biological plausibility have been argued above. While the term biological plausibility has been given an operational definition here, it is a term that is used in varying ways by different research communities. Therefore, the research question makes reference to the much more specific term retinotopic processing. The case for the importance of retinotopic processing has also been made above, and is reaffirmed by other researchers who have emphasized it as a means to maintain relevance to biology:

It is crucial to point out that this research question has not been answered previously. The question cannot have been addressed by any methods for visual homing which do not constraint themselves to retinotopic processing. Table 1.1 labels all methods which satisfy the constraints for retinotopic processing. Of these, neither Möller et al. [76] nor Möller [72] have yet been tested on a robot operating in an unstructured environment. Thus, there is no strong reason to believe they would perform competitively with the warping method in such an environment. This leaves Hafner [33] and Zeil et al. [108]. The performance of these two methods is shown in chapter 4 to be less than competitive with the warping method. Therefore, insofar 
as the literature review is reasonably complete, this research question has not been answered previously.

\subsection{Outline of Thesis}

Chapter 2 reviews the geometry of visual motion for an agent with a panoramic view of its enviromment. This chapter sets up important notation for subsequent chapters. Also, it describes qualitative properties of the flow field such as the focus of expansion and focus of contraction. These concepts will be crucial to developing one of the methods from chapter 7 and in analyzing the methods of chapter 9 .

Chapter 3 discusses the experimental framework used throughout the thesis. Most of the experiments were conducted on a database of images which is described in this chapter. The statistical tests performed on results from the image database are described here as well. Some online robot homing trials were performed in later chapters and the conditions for these are also described.

Chapter 4 reviews in depth three competing methods for visual homing. The methods reviewed in this chapter are those which have both a reasonable claim to biological plausibility, and have also been demonstrated to perform well on a robot in an unstructured environment. The three methods will be compared in their performance on the image database. In the end, Franz et al.'s warping method is selected to use for comparison in subsequent chapters

Chapter 5 describes how correspondence vectors can be mapped into home vectors. Correspondence vectors give the movement of features from the snapshot image to the current image. These features are expressed in the plane of the image, yet home vectors are expressed in the plane of travel (e.g. the ground). The one-dimensional technique of Hong et al. [43] is reviewed and compared with a new two-dimensional technique. Unlike Hong et al.'s technique, the two-dimensional method is able to generate correct home vectors from correct correspondence vectors without making any assumptions about the distribution of landmarks in the environment.

Chapter 6 extends Möller et al.'s neural snapshot model from a one-dimensional method applicable only in simulation, to a two-dimensional method applicable to real-world images. This method is found to perform reasonably well on the image database, but not competitively with the warping method. 
Chapter 7 introduces an image descriptor which is invariant to scale changes. Two homing methods are proposed based on this image descriptor. One of these exhibits performance that is nearly competitive with the warping method. Analysis of this method reveals that the scale invariant property is not crucial to its success. This result suggests that simpler image descriptors might suffice for visual homing.

Chapter 8 begins with such a descriptor. This descriptor is simply a block of local image pixels. A matching method based on this descriptor is found to exhibit performance that is more than competitive with the warping method. Analysis is carried out to determine if the complexity of this method can be reduced by further simplifying the image descriptor. This is found to be so and two simpler methods are proposed based on very minimal image descriptors. Both of these methods are found to perform to a reasonable standard and one of them performs competitively with the warping method.

Chapter 9 discusses differential-based techniques for finding correspondence between these same two minimal image descriptors. The use of differential techniques allows the search process to be avoided. Two methods are developed and although they both rely on a number of rather strong assumptions, several factors converge and allow them to succeed. They are found to be quite competitive with the warping method.

Finally, chapter 10 discusses the major conclusions and contributions of this work. It also describes a number of interesting proposals for future research. 


\section{Chapter 2}

\section{Spherical Flow Fields}

\section{$2.1 \quad$ Introduction}

In this chapter we present an analysis of the apparent motion or flow experienced by a moving agent which can see in all directions. If we have an agent translating and rotating in space with respect to some reference position, the agent's view of the world will change according to a vector field whose structure depends both upon the agent's motion and the structure of the environment. We assume here that the environment is projected onto the unit circle or sphere centred on the agent. We use the term 'flow' to refer to apparent motion in the image. Terms such as 'displacement' and 'movement' will refer to the motion of the agent itself within the enviromment.

This analysis will serve several purposes in the context of this thesis. It will be referred to in chapter 3 in the description of Franz et al.'s [26] warping method. It also sets up the notation that will be used in chapter 5 to describe vector mapping. Chapter 7 presents a homing method that is based upon finding the focus of contraction or focus of expansion of a spherical flow field-terms which are introduced here. Finally, in chapter 9 the properties of spherical flow fields will be critical in explaining the successful application of differential-based techniques to visual homing.

The next section will describe one-dimensional flow fields for an agent moving in the plane. This section will begin with the derivation of the equation for one-dimensional flow. Some approximations of this equation will permit us to describe the flow field's properties in a more qualitative fashion. These qualitative properties will then be illustrated by two examples. Following this we will proceed to the two-dimensional 
case and a final example of the qualitative properties of two-dimensional flow fields.

\subsection{Flow Fields in One Dimension}

\subsubsection{Mathematical Development}

The proceeding geometric analysis initially follows the development of Franz et al. [26]. We consider the motion of an agent in the plane. The agent's environment is projected onto the unit circle centred on the agent. The angular position of a point on this image circle is given by $\theta$, relative to the agent's current heading. Note that we adopt the somewhat unusual convention of positive clockwise angles, allowing a more natural mapping between the angle $\theta$ and the index for a column in a panoramic image corresponding to $\theta$.

Assume that the agent has moved from from its home position $\mathbf{s}$ to its current position $\mathbf{c}$. The agent's heading at $\mathbf{s}$ is given by the vector $\mathbf{v}_{\mathbf{s}}$, and its heading at $\mathbf{c}$ is $\mathbf{v}_{\mathbf{c}}$. To accomplish the move from $\mathbf{s}$ to $\mathbf{c}$ the agent would translate a distance $b$ in direction $\alpha$, and also rotate such that its total change in orientation is $\psi$. At position $\mathrm{s}$ a landmark $l$ is visible at angle $\theta .1$ is at a distance of $d_{\mathrm{s}}$ from $\mathrm{s}$. We idealize this landmark here as a point in space without further considering its visual aspect. After the move the view of 1 has shifted by $\delta$ to its new angular position $\theta+\delta$. Figure 2.1(a) summarizes the situation described thus far.

The angle $\delta$ is the flow and represents the change in image position of 1 . The goal now is to develop an expression for $\delta$ in terms of all the other quantities (i.e. in the form $\left.\delta=f\left(b, d_{\mathrm{s}}, \theta, a, \psi\right)\right)$. We begin by determining the angles within the triangle $\triangle$ slc. $\angle \mathrm{s}$ is easily determined as $\theta-a$. To obtain $\angle \mathrm{l}$ we add a line $\overline{\mathrm{sl}}$ parallel to $\overline{\mathrm{sl}}$ through $\mathbf{c}$ (this line is shown in figure 2.1(b)). The angle between $\overline{\mathrm{sl}}^{\prime}$ and $\overline{\mathrm{cl}}$ is the same as $\angle 1$. This observation allows us to obtain the following:

$$
\begin{aligned}
\angle \mathrm{l}+\theta & =\theta+\delta+\vartheta \\
\angle \mathrm{l} & =\psi+\delta
\end{aligned}
$$




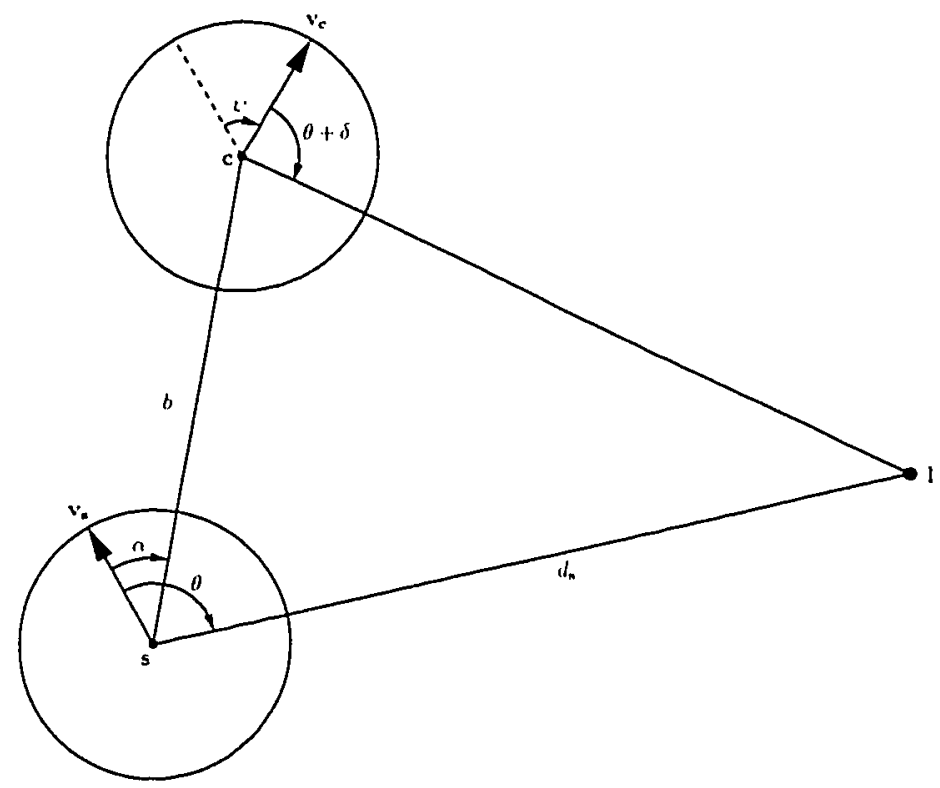

(a)

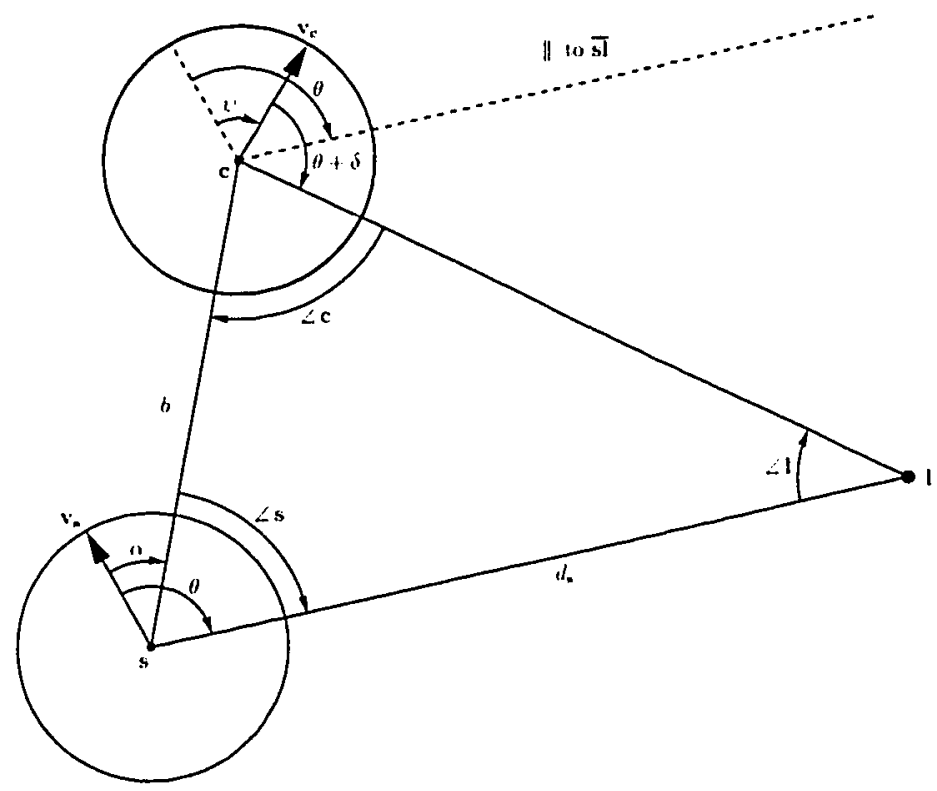

(b)

Figure 2.1: Motion of agent from $\mathrm{s}$ to $\mathrm{c}$ and the resultant flow of landmark $\mathrm{l}$. 
Given these two angles $\angle \mathrm{s}$ and $\angle \mathrm{l}$ we can obtain $\angle \mathrm{c}$.

$$
\begin{aligned}
& \angle \mathrm{c}=\pi-\angle \mathrm{s}-\angle \mathrm{l} \\
& \angle \mathrm{c}=\pi-(\theta-a)-\left(\psi^{\prime}+\delta\right) \\
& \angle \mathrm{c}=\pi-(\theta-a+\psi+\delta)
\end{aligned}
$$

The law of sines can now be applied.

$$
\begin{aligned}
\frac{d_{\mathbf{s}}}{\sin \angle \mathbf{c}} & =\frac{b}{\sin \angle 1} \\
\frac{d_{\mathbf{s}}}{\sin (\pi-(\theta-a+\psi+\delta))} & =\frac{b}{\sin (\psi+\delta)} \\
\frac{d_{\mathbf{s}}}{\sin (\theta-a+\psi+\delta)} & =\frac{b}{\sin (\psi+\delta)} \\
\frac{d_{\mathbf{s}}}{b} & =\frac{\sin (\theta-a+\psi+\delta)}{\sin (\psi+\delta)}
\end{aligned}
$$

The step from equation (2.1) to (2.2) is due to the fact that $\sin (\pi-a)=\sin a$. We now employ the addition formula for sines on equation (2.3).

$$
\begin{aligned}
& \frac{d_{\mathbf{s}}}{b}=\frac{\sin (\theta-a) \cos (\psi+\delta)+\cos (\theta-a) \sin (\psi+\delta)}{\sin (\psi+\delta)} \\
& \frac{d_{s}}{b}=\frac{\sin (\theta-\alpha) \frac{\cos \left(b^{2}+\delta\right)}{\sin \left(z^{\prime}+\delta\right)}+\cos (\theta-a) \frac{\sin \left(2^{\circ}+\delta\right)}{\sin \left(2^{2}+\delta\right)}}{\frac{\sin \left(b^{2}+\delta\right)}{\sin \left(b^{\circ}+\delta\right)}} \\
& \frac{d_{s}}{b}=\frac{\sin (\theta-a)}{\tan (\psi+\delta)}+\cos (\theta-\alpha) \\
& \tan (v,+\delta)=\frac{b \sin (\theta-a)}{d_{\mathrm{s}}-b \cos (\theta-a)} \\
& \delta=\arctan \left(\frac{b \sin (\theta-a)}{d_{\mathrm{s}}-b \cos (\theta-a)}\right)-\psi
\end{aligned}
$$

Equation (2.5) has the desired form. However, it is difficult to extract any qualitative insight from this rather complex expression. Therefore, we depart from Franz et al.'s analysis by positing an assumption which will allow $\delta$ to be defined more simply.

We assume that $d_{\mathbf{s}} \gg b$, meaning that the distance between the home and current position is small compared to the distance to the landmark. This assumption allows the denominator on the right-hand side of equation $(2.4)$ to be reduced to just $d_{s}$, 
yielding the following.

$$
\tan (\psi+\delta)=\frac{b}{d_{\mathrm{s}}} \sin (\theta-\alpha)
$$

If $d_{\mathrm{s}} \gg b$ then we also know that the angle $\psi+\delta$ will be small. To see this, consider moving 1 in figure 2.1(b) a large distance to the right. $\angle 1=\psi+\delta$ will shrink accordingly: Thus, given our initial assumption it is safe to assume that $\psi+\delta \ll \frac{\pi}{2}$. We can now exploit the approximation $\tan x \approx x$ for $x \ll \frac{\pi}{2}$.

$$
\begin{aligned}
\psi+\delta & =\frac{b}{d_{\mathrm{s}}} \sin (\theta-a) \\
\delta & =\frac{b}{d_{\mathrm{s}}} \sin (\theta-a)-\psi
\end{aligned}
$$

Equation (2.7) is sufficiently simple to allow some comments about the global properties of spherical motion fields to be made. These comments follow in the next section. Note that both Nelson and Aloimonos [80] and Hafner [33] have developed expressions equivalent to equation (2.7). However, both of these authors employed a differential analysis. The implicit assumption of these differential analyses is that the agent's motion is infinitesimal.

Equation (2.7) gives the relationship between the motion of an agent in the plane and the flow of a landmark 1 in the image. However, it is necessary to consider the flow of all landmarks across the whole image. If there exists a landmark at angular position $\theta$ then we can describe the flow of that landmark within the image as $\delta(\theta)$. In this case, $d_{\mathrm{s}}$ will also become a function of $\theta$.

$$
\delta(\theta)=\frac{b}{d_{\mathbf{s}}(\theta)} \sin (\theta-\alpha)-v
$$

This equation describes the flow of landmarks within the image resulting from a translational movement of distance $b$ in direction $\alpha$, plus rotation given by $\psi$. For a given environment and motion, equation (2.8) yields a one-dimensional flow field. A flow field is a vector field-in this case, a one-dimensional vector field. A flow field describes the apparent motion of patterns within the image. Our general assumption is that this apparent motion is generated by the agent's movement within the environment. 


\subsubsection{Example Flow Fields}

In order to appreciate the implications of equation (2.8) some examples of motion in two particular environments will be considered. Note that these examples were inspired by Nelson and Aloimonos's description of spherical flow fields (which they refer to as spherical motion fields) [80].

We begin with an example of purely translational motion in a circular environment. Figure 2.2(a) depicts an agent facing upwards at position $s$ in the centre of a ring. The agent then translates upwards and to the right at an angle of $\alpha=\frac{\pi}{4}$. The radius of the world is unity and the distance of travel is $b=0.28$. The flow field is given as the heavier trace in figure 2.2(c). A rough graphical depiction of the flow field which we will refer to as the flow annulus is shown centred on the agent's new position at c. On this annulus clockwise arrows indicate positive $\delta$ values, whereas counterclockwise arrows indicate negative values.

At $\theta=\frac{\pi}{4}$ in the flow field $\delta$ switches from negative to positive. This represents the focus of expansion (FOE). On the flow annulus the arrows diverge outwards at the FOE. At $\theta=\frac{5 \pi}{4}$ the flow field switches back to negative. This represents the focus of contraction (FOC). The FOC is also indicated on the flow amnulus at the point where the arrows converge. As the agent's enviromment is just a ring, $d_{\mathbf{s}}=1$ for all $\theta$. Therefore, the amplitude of the flow field is exactly sinusoidal with a peak value of 0.28 .

Figure 2.2(b) shows a second example where $d_{\mathrm{s}}$ is dependent on $\theta$. The agent's initial position $\mathrm{s}$ lies near the bottom right corner of a rectangular box of width five and height nine. The inset graph in figure 2.2(b) gives the value of $d_{\mathbf{s}}(\theta)$ at. $\mathrm{s}$. This distance profile will result in a different flow field than for the agent in the ring. However, we shall see that there are important properties of the field which remain the same. The translational movement from $\mathbf{s}$ to $\mathbf{c}$ is again in the direction $a=\frac{\pi}{4}$, although the distance moved is now $0.7^{1}$.

The flow field for this movement is depicted as the heary bottom-most trace in figure 2.2(d). As both the flow field and flow amnulus show, the FOE is again at $\frac{\pi}{4}$ and the FOC at $\frac{5 \pi}{4}$. The amplitude of the flow field now varies from its sinusoidal envelope, yet its overall appearance is relatively unchanged.

\footnotetext{
${ }^{1}$ These distances were chosen according to the proportions of the diagrams in figures 2.2(a) and $2.2(b)$.
} 
In both environments the flow field can be separated into two disjoint regions. One region exhibits positive $\delta$ and clockwise flow, while the other exhibits negative $\delta$ and counterclockwise flow. The flow field is split evenly in two between these two regions and the foci are defined as the two points where the regions meet. The FOE is aligned with the direction of translation and the FOC is $180^{\circ}$ opposite. Although not demonstrated here, it is clear from equation (2.8) that the flow generated by distant objects will be very small. The sign of the flow of such objects, however, will still be consistent with the characterisation given above. Objects at an effectively infinite distance will contribute nothing to the flow field. The presence of such objects will create 'holes' in the flow, possibly limiting the ability to detect the direction of translation with precision.

These properties characterize spherical motion fields resulting from pure translation. The lesson we wish to extract is that the overall structure of the flow field is dependent more so on the agent's movement, than the layout of the enviromment. This is fortunate because it allows us to infer the agent's movement from the flow field without knowing anything about the enviromment.

The properties of the flow field change, however, in the presence of rotation. As equation (2.8) shows, the rotational component $\psi$ is simply added to the sinusoidal component, shifting it up or down. Figures 2.2(c) and 2.2(d) both show two additional traces for $\psi=\frac{\pi}{18}$ and $\psi=\frac{\pi}{9}$. For $\psi=\frac{\pi}{18}$ the overall structure of the flow field has changed significantly. The FOE and FOC are still represented by the crossings of the graph with the $x$-axis. However, these two foci are no longer separated by $180^{\circ}$. For $\psi=\frac{\pi}{9}$ there are no crossings with the $\mathrm{x}$-axis. Hence, the FOE and FOC no longer exist and all of the flow is of the same sign.

\subsection{Motion Fields in Two Dimensions}

\subsubsection{Mathematical Development}

Equation (2.8) describes the flow within a plane. We can extend our analysis to two-dimensional flow by studying the flow that occurs within two planes. The first plane is $\mathcal{P}$, which is our agent's plane of travel. It is useful to imagine our agent as a wheeled robot with the plane $\mathcal{P}$ parallel to the floor at the height of the robot's camera. Plane $\mathcal{P}$ is formally defined by the goal position $\mathbf{s}$, the current position $\mathrm{c}$, 


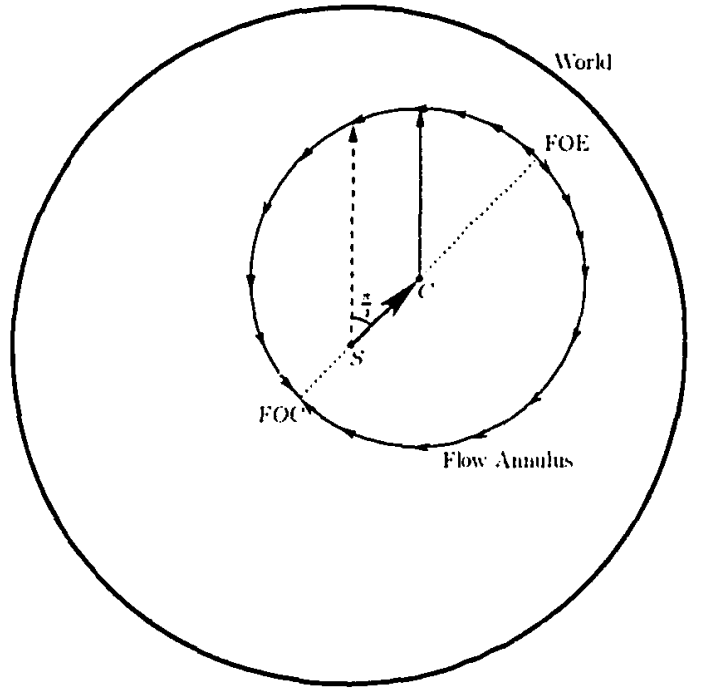

(a)

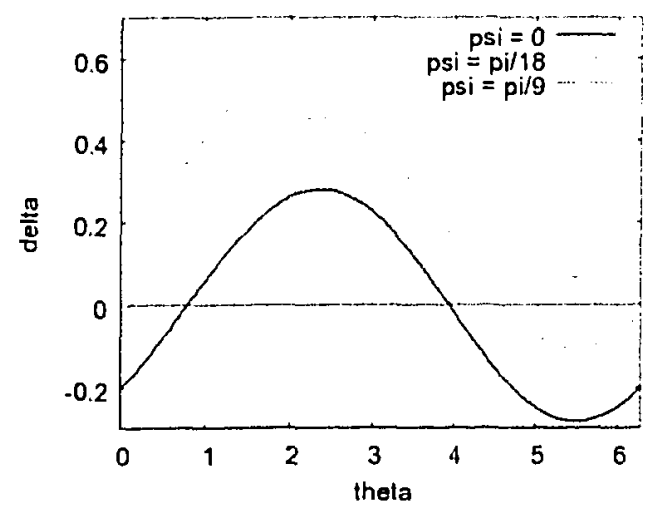

(c)

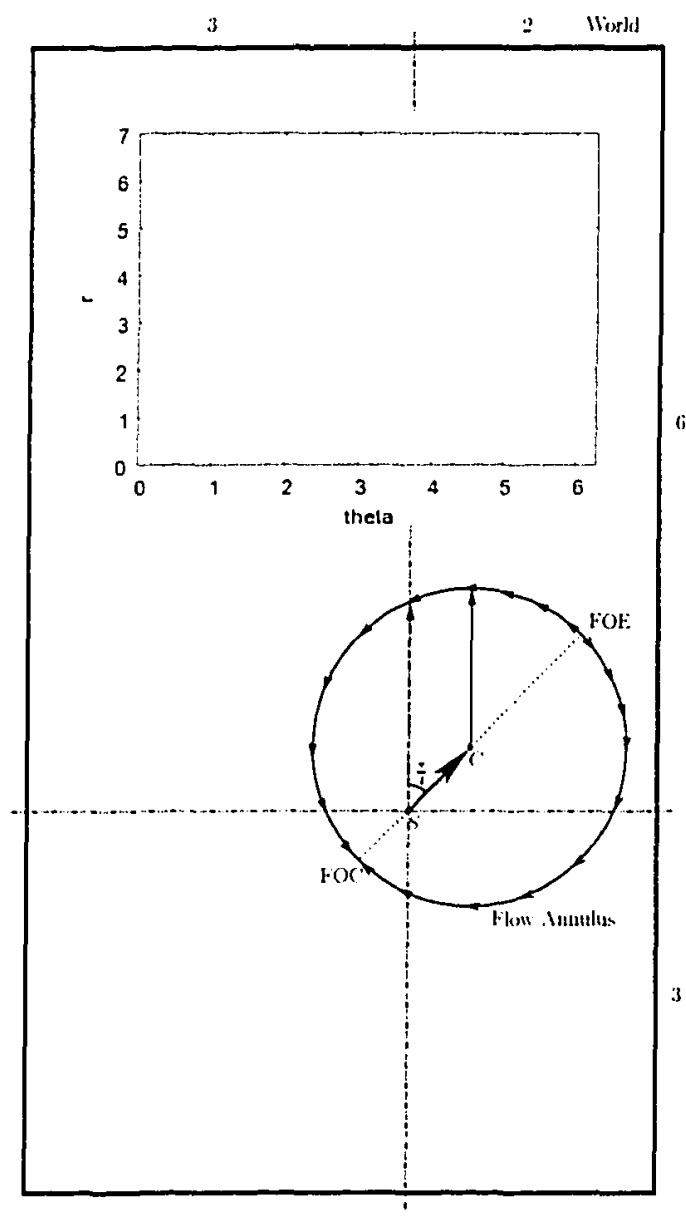

(b)

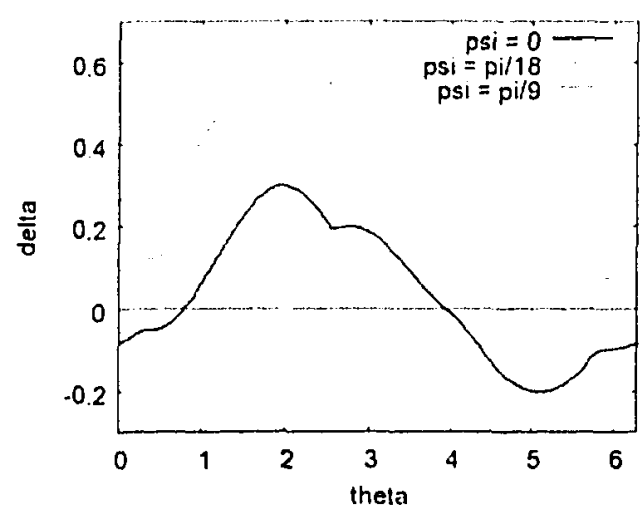

(d)

Figure 2.2: Motion of an agent within two different enviromments. 
and some in-plane landmark 1 . Now consider another landmark $\mathrm{l}^{\prime} \notin \mathcal{P}$. The points $\mathbf{s}, \mathbf{c}$, and $\mathbf{l}^{\prime}$ define a new plane $\mathcal{P}^{\prime}$.

Similar to section 2.2 we define angles to measure how $\mathrm{l}^{\prime}$ shifts as the agent moves from $\mathbf{s}$ to $\mathbf{c}$. However, these angles should not be measured relative to the agent's heading $\mathbf{v}$, as $\mathbf{v} \notin \mathcal{P}^{\prime}$. Instead we measure angles with respect to the projection of the agent's heading onto the intersection line between $\mathcal{P}$ and $\mathcal{P}^{\prime}$. The projected heading of the agent at $\mathbf{s}$ is $\mathbf{v}_{\mathbf{s}}^{\prime}$, where $\mathbf{v}_{\mathbf{s}}^{\prime} \| \overline{\mathbf{s c}}$. Similarly, the projected heading at $\mathbf{c}$ is $\mathbf{v}_{\mathbf{c}}^{\prime}$. In section 2.2 the angle $\alpha$ gave the direction of translation with respect to $\mathbf{v}_{\mathbf{s}}$. As the direction of translation is parallel to $v_{s}^{\prime}$, the equivalent angle $\alpha^{\prime}$ for plane $\mathcal{P}^{\prime}$ is zero. Also, because $\mathbf{v}_{\mathbf{s}}^{\prime} \| \mathbf{v}_{\mathbf{c}}^{\prime}$, the rotation angle $\psi^{\prime}$ is zero as well. The angles $\theta$ and $\delta$, however do have non-zero equivalents in $\mathcal{P}^{\prime}$. At $\mathbf{s}$ landmark $\mathbf{l}^{\prime}$ is at angle $\theta^{\prime}$, and at $\mathrm{c}$ is at $\theta^{\prime}+\delta^{\prime}$. Figure 2.3 shows these angles.

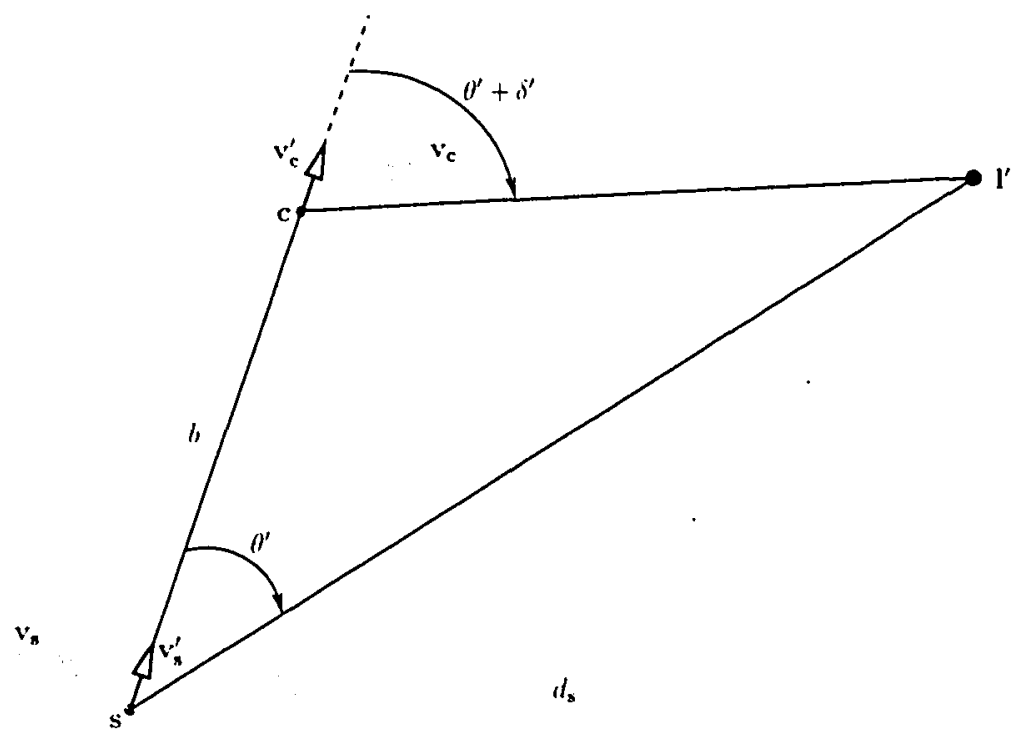

Figure 2.3: Motion of agent from $\mathrm{s}$ to $\mathrm{c}$ and the resultant flow of landmark $\mathrm{l}^{\prime}$ within plane $\mathcal{P}^{\prime}$. Entities exclusively within plane $\mathcal{P}$ appear in grey.

Equation (2.8) can now be adapted for plane $\mathcal{P}^{\prime}$

$$
\delta^{\prime}\left(\theta^{\prime}\right)=\frac{b}{d_{\mathbf{s}}\left(\theta^{\prime}\right)} \sin \left(\theta^{\prime}\right)
$$


The above equation describes the flow perceived by a moving agent for a landmark $\mathbf{l}^{\prime}$ in a plane $\mathcal{P}^{\prime}$ that intersects the line of motion $\overline{\mathbf{s c}}$.

\subsubsection{Example Flow Field}

As for the one-dimensional case, we provide an example to illustrate two-dimensional flow. In this case, it is convenient to use a graphical simulation of an agent with a panoramic imaging system. The agent is placed at the centre of a sphere. On the surface of the sphere are markers with unique grevscale colours for identification. The markers are placed along great circles of the sphere which intersect at two poles. Positions $\mathbf{s}$ and $\mathbf{c}$ lie on the line connecting the two poles. Hence, the great circles represent the intersection of planes with the sphere where these planes are parallel to the direction of motion. Figure 2.4 illustrates the positions of $\mathbf{s}$ and $\mathbf{c}$ within the sphere and shows both the markers and the great circles upon which they' lie.

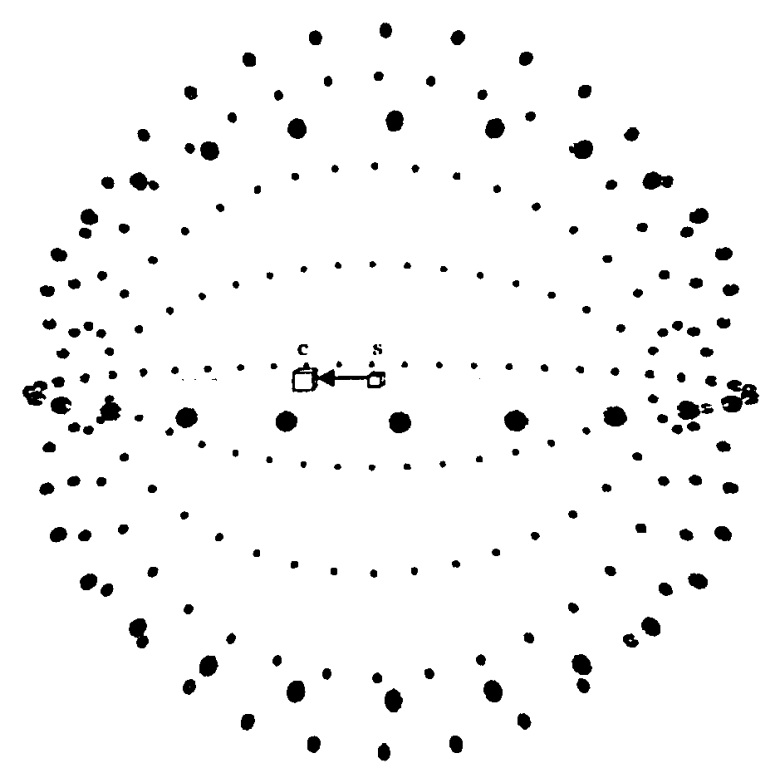

Figure 2.4: Outside perspective view of the sphere visualized in OpenGL. The positions of markers along the great circles are indicate by small shaded spheres. Position $\mathbf{s}$ is shown as a small cube at the sphere's centre. Position $\mathbf{c}$ is the larger cube.

Figures 2.5(a) and 2.5(b) shows panoramic images captured by the agent at $\mathrm{s}$ and at $\mathbf{c}$. The sphere is visualized using OpenGL. The views normally produced by 
OpenGL are standard perspective views. To produce a panoramic image, views are taken from the same position at orientations of $0^{\circ}, 72^{\circ}, 144^{\circ}, 216^{\circ}$, and $288^{\circ}$. These five views are then stitched together horizontally. Perspective distortion is removed by re-projecting the images onto a cylinder using the method of [67]. This cylindrical projection allows the five images to fit together seamlessly:

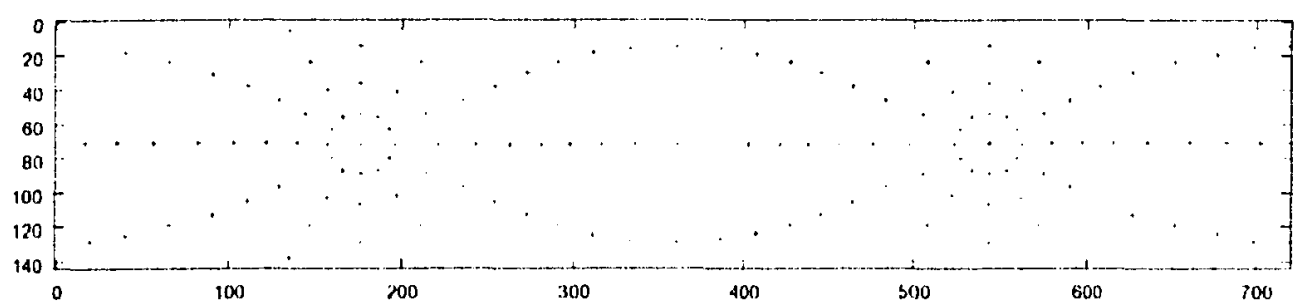

(a)

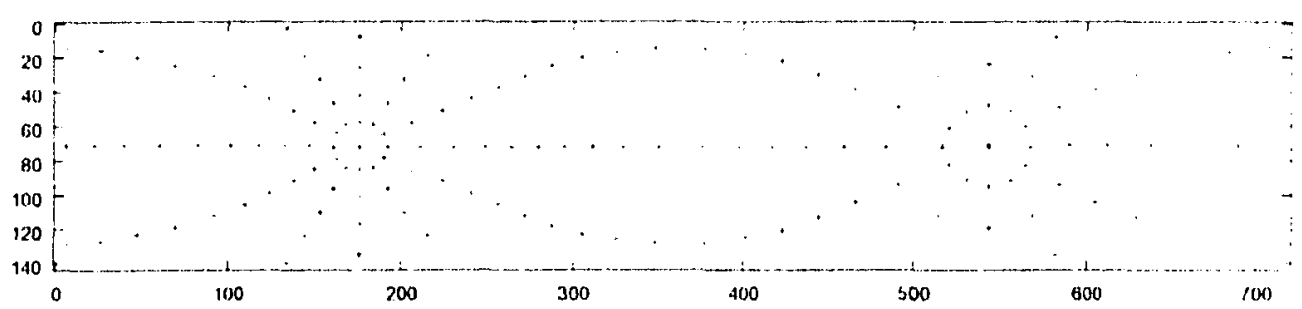

(b)

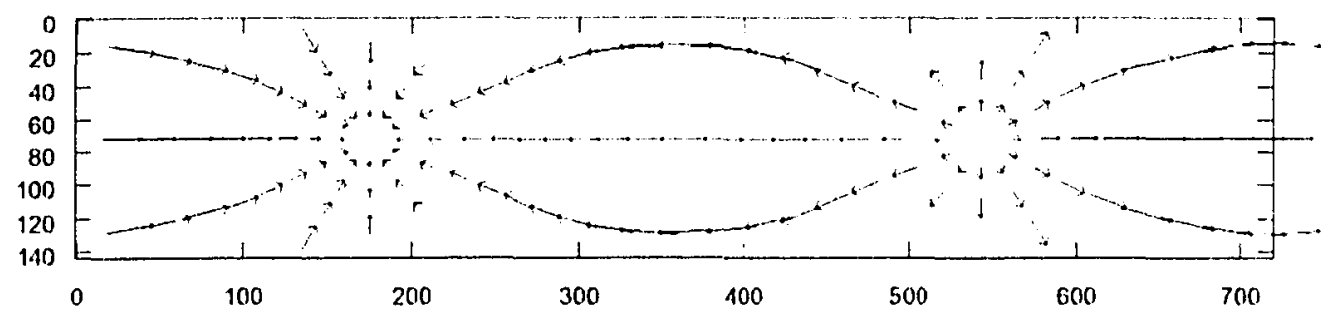

(c)

Figure 2.5: Panoramic images taken from within a sphere at $\mathbf{s}$ (a) and $\mathbf{c}(\mathrm{b})$, and the correspondence vectors between them (c). See text for further details.

We refer to the panoramic image captured at $\mathbf{s}$ as $S$, and that captured at $\mathbf{c}$ as $C$. Correspondences between these two images are found by matching the coloured markers in $S$ to those in $C$. Figure 2.5(c) shows correspondence vectors with tails at markers in $S$ and heads at the corresponding markers in $C$.

It is clear from figure 2.5 (c) that all flow is confined to planes parallel to the 
direction of motion. Within each of these planes the flow is dictated by equation (2.9). It is also clear that the FOE and FOC are characteristic of two-dimensional flow fields as well as one-dimensional fields. Again, these foci are $180^{\circ}$ apart and delineate regions of clockwise flow from counterclockwise flow within the planes. Further, flow approaches zero at the foci and reaches peak value at points orthogonal to the foci. Although not demonstrated here, it is again the case that the separation between foci can be altered by rotation and that very distant objects can create 'holes' in the flow field.

All of the above-mentioned properties are inherent in equation (2.9) which describes the flow within planes parallel to the direction of motion as having a sinusoidal envelope.

\subsection{Conclusions}

This chapter has introduced some of the geometry and notation which will be important in later chapters. Also of key importance were the descriptions of the qualitative properties of spherical flow fields. 


\section{Chapter 3}

\section{Experiments and Methodology}

\subsection{Introduction}

This chapter details the experimental approach to be taken throughout this thesis. The goal is to describe how to measure a homing method's performance. The primary data on which to measure performance is the image database. This is a set of images collected within a variety of unmodified indoor environments under various conditions. Two performance metrics and various experiments are described to obtain results on the image database. To validate the results on the image database a limited number of robot homing trials were also conducted. The final section describes how these trials were carried out.

\subsection{Image database}

A database of images was collected in the robotics laboratory of the Computer Engineering Group of Bielefeld University. Images were collected by a camera mounted on a robot and pointed upwards at a hyperbolic mirror. The camera was an ImagingSource DFK 4303. The robot was an ActivMedia Pioneer 3-DX. The mirror was a large wide-view hyperbolic mirror from Accowle Ltd. Figure 3.1 show images of the robot, and the camera and mirror assembly.

The room was unmodified except to clear the floor and apply controlled additions to the original environment, as described below. Images were collected by positioning the robot at 10 start positions spaced $30 \mathrm{~cm}$ apart along a line perpendicular to 


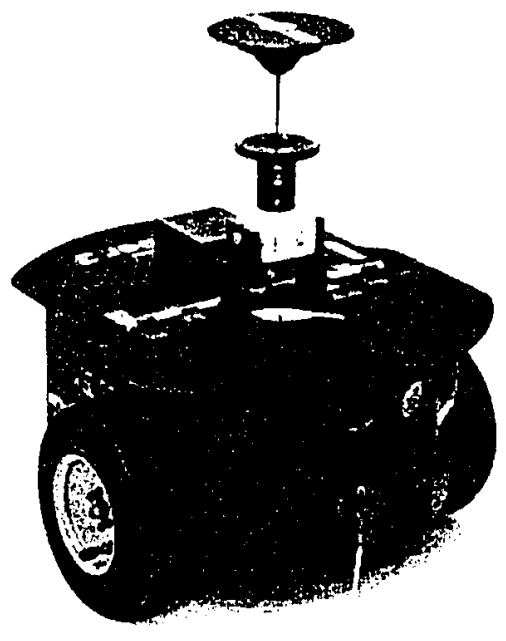

(a)

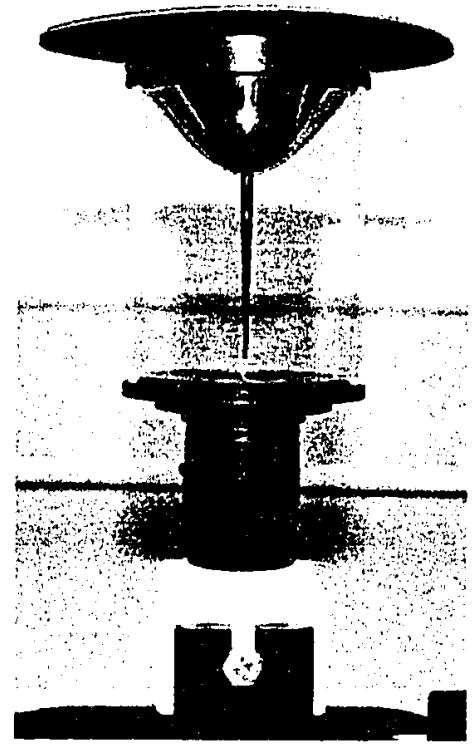

(b)

Figure 3.1: (a) The ActivMedia Pioneer 3-DX robot with attached camera and hyperbolic mirror. (b) A close-up of the camera and hyperbolic mirror assembly.

the long axis of the room. At each start position the robot was manually oriented to parallel the long axis of the room. This orientation was facilitated by using two laser pointers mounted to the robot's chassis. The robot would be placed at each start position such that both laser beams touched specially placed marks on the two opposing walls. After manual positioning the robot's capturing cycle would be activated via wireless ethernet. At the start of the capturing crcle the robot would capture an image from the start position. It would then move alhead $30 \mathrm{~cm}$, stop, capture another image, and continue in this manner until it had reached the other side of the room. The positions at which images were captured define a capture grid of dimensions $2.7 \mathrm{~m}$ by $4.8 \mathrm{~m}$, which covered nearly all of the floor's free space.

The images were captured and stored at the camera's highest resolution $(752 \times$ 564). A hyperbolic mapping was then applied to produce panoramic images. These panoramic images were then low-pass filtered (butterworth filter: relative width 0.25 , order 1) in preparation for downsampling. The low-pass filtered images were downsampled to a relatively low resolution $(206 \times 46)$. This level of resolution is roughly 
consistent with that of honeybee eyes. Land [60] reviews the visual acuity data of many insects and presents four differing estimates of Apis mellifera (honeybee) interommatidial angles. Larger interommatidial angles give lower resolution images. The low resolution used here is approximately consistent with the largest interommatidial angle reported by Land $\left(\Delta \phi=1.7^{\circ}\right)$.

Images were collected with the room in a number of different conditions. The names and descriptions of these image collections are as follows:

original The standard or default condition of the room. Images were collected with both overhead fluorescent light bars on, the curtains and door closed, and no extraneous objects present.

chairs Three additional office chairs were positioned within the capture grid.

arboreal A tall $(3 \mathrm{~m})$ indoor plant was added to the centre of the capture grid.

screen A projection screen (height $2.55 \mathrm{~m}$ ) was added to the centre of the capture grid. The screen ran parallel to the long axis of the room.

day Images were collected with curtains open in full daylight.

twilight Curtains and door open. Image collection began at 18:34 on March 13, 2004 just after sunset ${ }^{1}$ with the room still receiving plenty of daylight. The last image was captured at 19:14. The sky outside was quite dark by this time.

doorlit The light bar near the window was switched off.

winlit The light bar near the door was switched off.

Figure 3.2 shows sample images from all of the above collections. Henceforth, we will refer to images from these collections as being from the office environment.

Images were also collected from two locations within the main hall of Bielefeld University. These locations were categorically different from the office environment in that the distances to viewed objects ranged from several metres to hundreds of metres. These image collections are larger, spanning $4.5 \mathrm{mby} 10 \mathrm{~m}$ in area at $50 \mathrm{~cm}$

\footnotetext{
${ }^{1}$ The official time of sunset on that day was 18:32 for Osnabrïck Airport, approximately $50 \mathrm{~km}$ away [13]
} 
resolution. All images were captured late at night when the hall was free of people. Two sets of images were collected:

hall1 Capture grid was adjacent to a dining area with two large open regions extending off in opposite directions, and the large main hall extending out opposite the dining area. Overhead structures are visible and appear in significantly different positions within the collected images.

hal12 Capture grid was near a message board at approximately the centre of the hall.

Figure 3.3 shows sample images from these two collections.

This image clatabase is available for public download at http://www.ti.uni-bielefeld.de/html/research/avardy.

\subsection{Experiments}

\subsubsection{Assumptions}

\section{Orientation}

The images from the database described above were all taken from approximately the same orientation. Thus, insofar as the experiments on this database emulate the behaviour of a robot, it is assumed that this robot has some means of correcting for changes in orientation. This allows us to propose methods that assume that the snapshot and current images were taken from the same orientation. The discussion in section 1.2.4 suggests that the visual homing methods used by insects are also based on the assumption that the orientation held at the goal is recovered to support homing.

The problem of correcting for changes in orientation can be solved by a variety of special sensors. One obvious orientation sensor is a magnetic compass. Magnetic compasses are cheap but can be umreliable in indoor enviromments (see section 3.4). Orientation can be maintained by path integration but will be subject to cumulative error and other problems. Borenstein et al. [4] discuss a range of techniques and devices for accurately estimating orientation. As described in section 1.2.2 insects are able to estimate orientation through both magnetic and skylight compasses. Orientation can also be recovered through visual means alone. Both Zeil et al. [108] and Franz et al. [26] discuss techniques for recovering orientation by comparing the snapshot and current images. These techniques will be described further in chapter 4 . Various other means for recovering orientation by vision have been proposed $[89,28]$. 

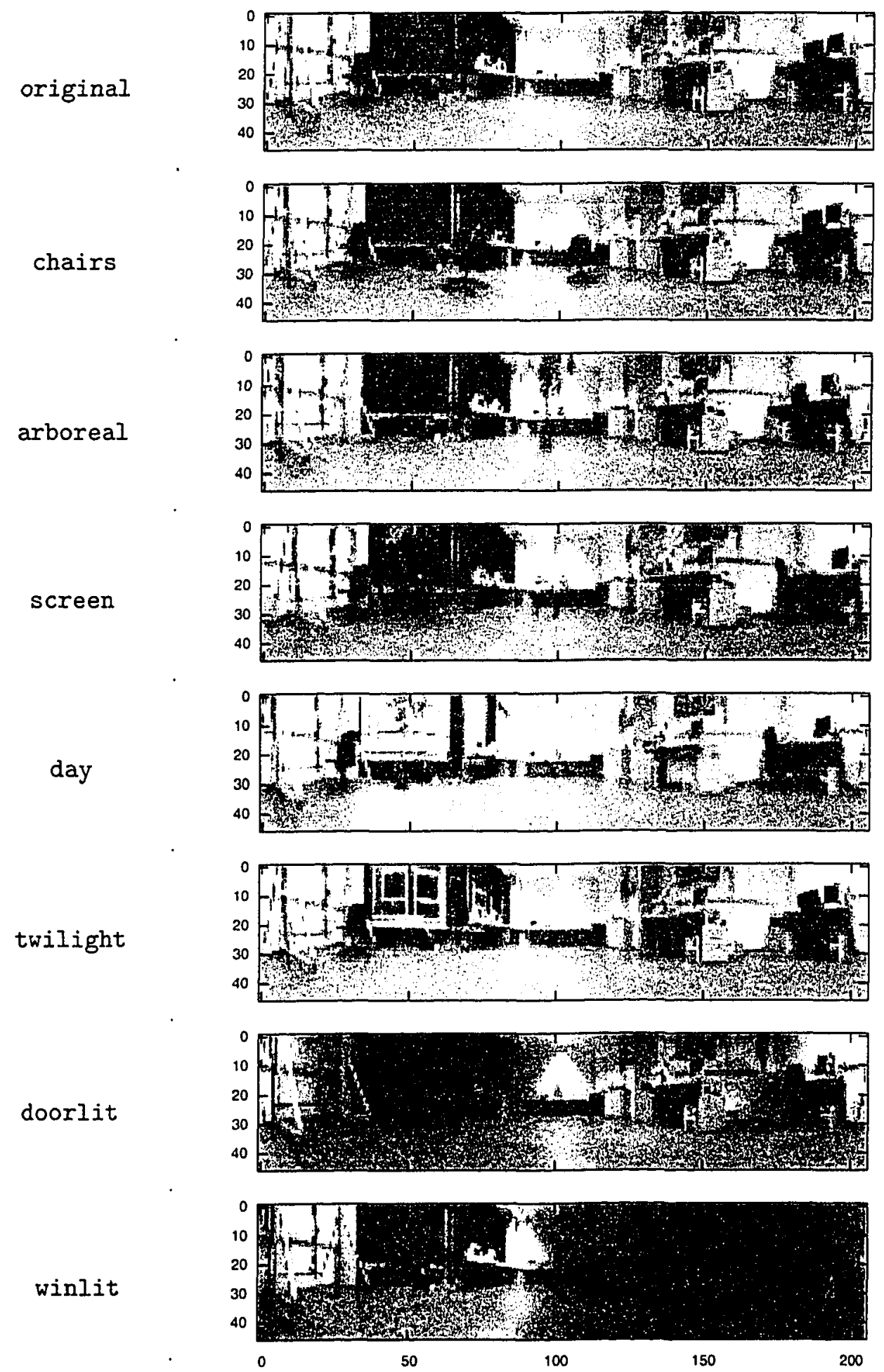

Figure 3.2: Sample images from eight image collections in the office environment. Images taken from position $(3,0)$. 
hall1

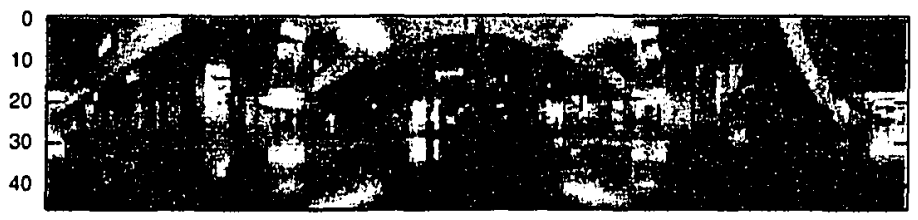

hall2

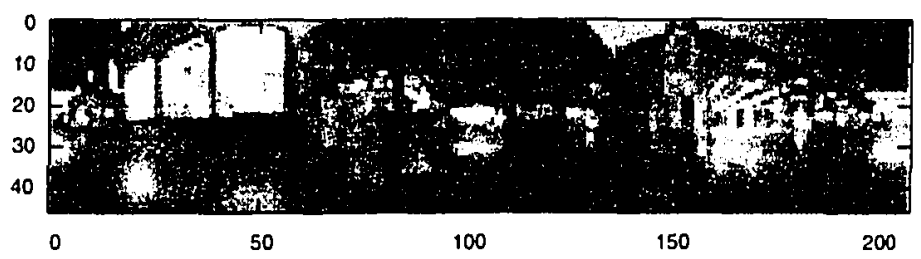

Figure 3.3: Sample images from two image collections in the hall environment. Images taken from position $(5,10)$.

\section{Normalized Home Vectors}

Only the direction of home vectors will be of interest for the experiments below. Some homing methods such as Franz et al.'s [26] and Röfer's [89] compute approximate measures of the distance to the goal and can therefore vary the size of incremental movements to aroid overshooting the goal. The method employed by Röfer is to sum the lengths of all estimated correspondence vectors. This measure is applicable to nearly all of the methods that will be presented in this thesis. Another possible measure would be the sum of squared differences between the snapshot and current images ${ }^{2}$. As there exists several simple strategies to roughly estimate distance to the goal we will not further consider the issue. Instead all home vectors will be normalized to unit length.

\subsubsection{Home Vectors for a Single Snapshot Position}

To assess a homing method on a particular image collection, the following procedure is used. A single image from the image collection is selected as the snapshot image. The coordinates of this image within the capture grid are taken as the goal coordinates. The snapshot image is then fed into the homing method. The homing method may or may not apply some processing to the snapshot image prior to storing it. All remaining images are then input, in turn, to the homing method. For each of these images the homing method will return a home vector. All home vectors are stored for analysis.

\footnotetext{
${ }^{2}$ Zeil et al. [108] use this measure to cletermine the direction of homing as well. Their method is reviewed in chapter 4 .
} 
To illustrate the experiments and methods of analysis described in this chapter three demonstration homing methods are employed. The first is the ideal homing method which always returns correct home vectors. The home vectors computed by this method are given by

$$
\mathrm{v}_{\text {Ideal }}=\frac{\mathrm{s}-\mathrm{c}}{\|\mathrm{s}-\mathrm{c}\|}
$$

Clearly this is not a realizable homing method as $\mathbf{s}$ is unknown with respect to $\mathrm{c}$.

The second and third demonstration methods are noisy variants of the ideal method. They return home vectors that are distorted from the ideal by a particular noise model. The first such noisy method corrupts each home vector with impulse noise. With probability $p_{\text {Impulse }}$ an ideal home vector will be reset to the following:

$$
\mathbf{v}_{\text {Impulse }}=\left(\begin{array}{l}
\cos \beta_{\text {ImpuIse }} \\
\sin \beta_{\text {Impulse }}
\end{array}\right)
$$

where $\beta_{\text {Impulse }}$ is chosen from the range $[0,2 \pi]$ with uniform probability. Note that a new value of $\beta_{\text {Impulse }}$ will be randomly selected for each corrupted home vector.

The second noisy method corrupts all home vectors with Gaussian noise. Let $\beta$ be the angle of the true home vector. Home vectors from this method are set to

$$
\mathbf{v}_{\text {Gauss }}=\left(\begin{array}{l}
\cos \left(\beta+\gamma_{\text {Gauss }}\right) \\
\sin \left(\beta+\gamma_{\text {Gauss }}\right)
\end{array}\right)
$$

where $\gamma_{\text {Gauss }}$ is drawn from a Gaussian clistribution with mean 0 and standard deviation $\sigma_{\text {Gauss }}$. A new value of $\gamma_{\text {Gauss }}$ will be randomly selected for every home vector.

We will refer to the three demonstration methods as Ideal, Impulse, and Gauss. For Impulse the parameter $p_{\text {Impulse }}$ will be set to 0.1 . For Gauss the parameter $\sigma_{\text {causs }}$ will be set to $0.1 \pi$.

Figure 3.4 shows home vectors generated by these three methods. The home vector at each grid position is the homing method's output on the corresponding image. Of course, for these demonstration methods no real processing occurs and the characteristics of the input image are irrelevant.

\subsubsection{Performance Metrics}

If one was shown the home vectors in figure 3.4 and told that the goal was at $(6,4)$, it would be clear that the method of figure 3.4(a) was superior to the other two. To distinguish the other two home vector fields it is necessary to establish some performance metrics. 


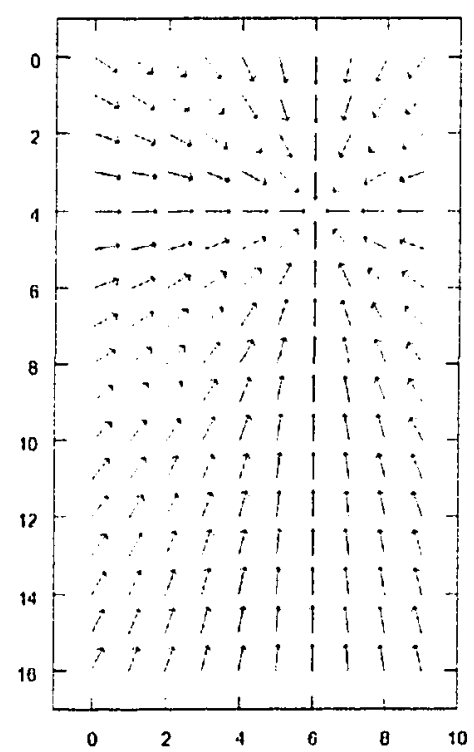

(a) Ideal:

$A A E_{(6,4)}=0$, $R R_{(6,4)}=1$

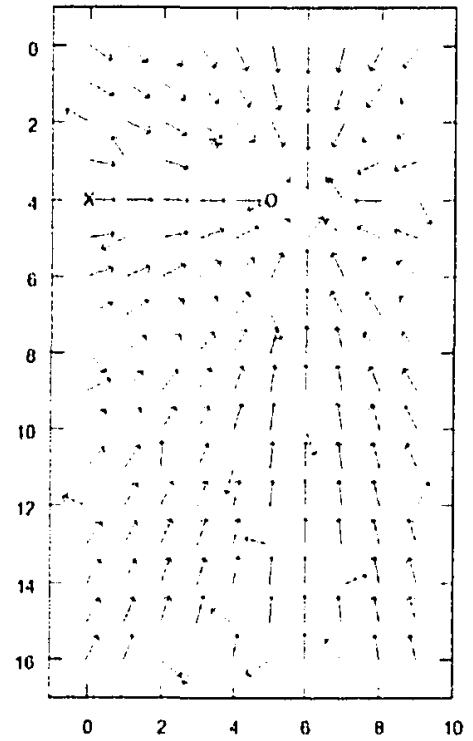

(b) Impulise: $A A E_{(\mathrm{i}, 4)}=0.165$, $R R_{(\mathrm{i} .4)}=0.889$

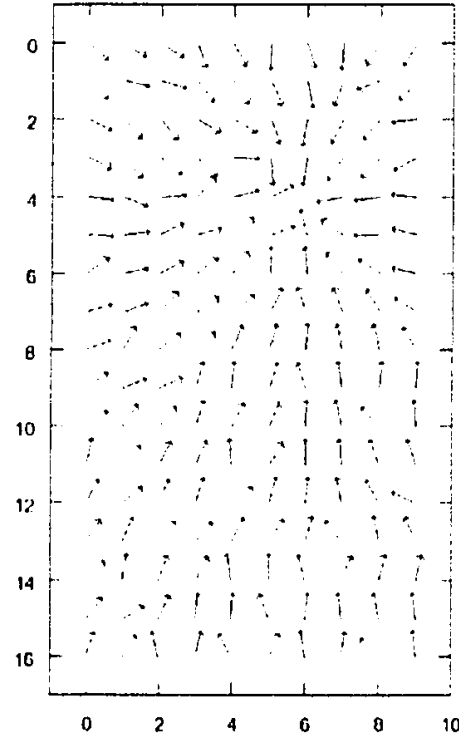

(c) Gauss: $A A E_{(6.4)}=0.255$, $R R_{(6,4)}=0.986$

Figure 3.4: Home vector fields for the ideal homing method and two noisy methods for snapshot position $(6,4)$ of image collection original. The labels ' $X$ ' and ' $O$ ' in (b) are referred to in the text.

\section{Average Angular Error}

Perhaps the simplest means of measuring homing performance is to compute the averagc angular error between the observed home vectors and the true home vectors. For analysis purposes, we know the coordinates of both the current position $\mathbf{c}$, and the goal position s. Therefore $\mathbf{v}_{\text {Ideal }}$ is known. Given a homing method, Method, which computes a home vector $\mathbf{v}_{\text {Method, }}$, the angular error $(A E)$ is defined as

$$
A E(\mathbf{s}, \mathbf{c})=\arccos \left(\mathbf{v}_{\text {Ideal }}(\mathbf{s}, \mathbf{c}) \cdot \mathrm{v}_{\text {Method }}(\mathbf{s}, \mathbf{c})\right)
$$

measured in radians.

The average angular error (AAE) is computed for a single snapshot position s by averaging over all current positions. For an image collection of width $m$ and height $n, A A E$ for 
snapshot position $\mathbf{s}$ is defined as

$$
A A E_{\left(s_{x}, s_{y}\right)}=\frac{1}{m n-1} \sum_{c_{r}=0}^{m-1} \sum_{c_{y}=0}^{n-1} A E\left(\left(\begin{array}{l}
s_{x} \\
s_{y}
\end{array}\right),\left(\begin{array}{l}
c_{x} \\
c_{y}
\end{array}\right)\right)
$$

likewise measured in radians. Note that the angular error at the snapshot position is excluded from this summation. $A A E$ is a useful performance metric because it is easy both to understand and to compute. It has also been employed by other robot homing studies $[104,33]$.

\section{Return Ratio}

In the biological literature, homing success has been described in terms of catchment area [7]. The catchment area is the area surrounding the goal within which the agent is able to successfully home. Average angular error is an indirect measure of catchment area. It is apparent that for a perfect homing field with zero angular error (e.g. figure 3.4(a)) that the catchment area covers the entire capture grid. However, while average angular error is invariant to the location of errors, the size of the catchment area will depend very much on where errors occur.

Consider an agent placed at position $(0,4)$ in figure 3.4 (b) (this position is marked by an ' $\mathrm{X}$ '). If homing according to the home vectors in that figure, the agent will travel right towards the goal until position $(5,4)$ (marked by an ' $O$ ') is encountered. At this point the agent will begin oscillating left and right. Depending on the parameters of its motion, it may work itself free and continue towards the goal. However, it may remain stuck indefinitely. In this case, the one incorrect vector at position $(5,4)$ would block the progress of agents starting at positions $(0,4)$ through $(5,4)$. Therefore, these positions would be excluded from the catchment area.

To account for the position of errors and to provide a more direct measurement of catchment area, a simulation of homing is performed. A dummy agent is placed at all integer grid positions and allowed to move according to the home vectors which have been pre-computed for the method in question. The agent moves in steps of length $l_{\mathrm{rr}}$. The home vector for the integer grid position closest to the agent's current position dictates the agent's movement. The outcome of the homing attempt is binary. If the agent reaches within a clistance of one to the goal position then the attempt is considered successful. The total distance that the agent is allowed to travel is set to half the circumference of the capture grid. For image collections from the office environment this is $9+16=25$. If the agent travels this distance but has not yet reached the goal then the attempt is considered 
unsuccessful. This technique is similar to those described by other robot visual homing studies [26] [95].

The output from the above procedure is a binary map of successful vs. unsuccessful homing attempts for all start positions. The return ratio is computed by counting the number of successful positions and dividing by the total number of homing attempts (10 $\mathrm{x}$ $17-1=169$ for the office environment). We indicate the return ratio for snapshot position $(x, y)$ as $R R_{(x, y)}$. This quantity is in the range $[0,1]$, where 1 indicates perfect homing.

The step length parameter, $l_{\text {rr }}$, represents the clistance that a robot travels after processing an image. For the robotic trials referred to below the robot travels $50 \mathrm{~cm}$ after processing an image. The spacing of the capture grid is $30 \mathrm{~cm}$, thus $l_{\mathrm{rr}}=1.66 \mathrm{t}$ would represent this situation. Figure 3.5 shows homing attempts computed for the home vectors in figure 3.4(b) with varying settings for $l_{\mathrm{rr}}$. For $l_{\mathrm{rr}}=1.667$ (shown in figure $3.5(\mathrm{a})$ ) the problem clescribed above for the erroneous vector at position $(5,4)$ does not occur. This is because the step size is large enough that the problem location is simply jumped over. For smaller step sizes the bad vector at $(5,4)$ has more influence-positions to the left of $(5,4)$ are no longer included in the catchment area. However, these smaller step sizes create artefacts as well. It is noticeable in figure $3.5(\mathrm{e})$ that the agent sometimes travels along the border between cells of the capture grid. This behaviour is unrealistic for real robot homing. Thus, a moderate step size, $l_{\mathrm{rr}}=0.5$ has been chosen for the experiments in this thesis.

The captions in figure 3.4 show the computed values of the two performance metrics described above. Gauss exhibits higher $R R$ than Impulse. Thus, Gauss would yield a larger catchment area, even though its $A A E$ is higher (recall that good performance is indicated by low $A A E$ and high $R R$ ).

\subsubsection{Performance Across Snapshot Position}

Homing performance is dependent on the chosen snapshot position. To assess this dependence we test homing methods on a sampling of 20 snapshot positions. Figure 3.6 shows these snapshot positions which were chosen to evenly sample the capture grid.

Performance metrics $A A E$ and $R R$ are each computed for all 20 snapshot positions. To obtain an overall measure of homing performance according to these two metrics the metric's value is averaged across all snapshot positions. This is indicated as either $A . A E_{*}$ or $R R_{*}$. To specify that such a value was obtained on a particular image collection, this collection is specified (e.g. $A A E_{*}$ (original)). However the image collection will not be specified if it is clear from the surrounding context. A further addition to this notation occurs in 


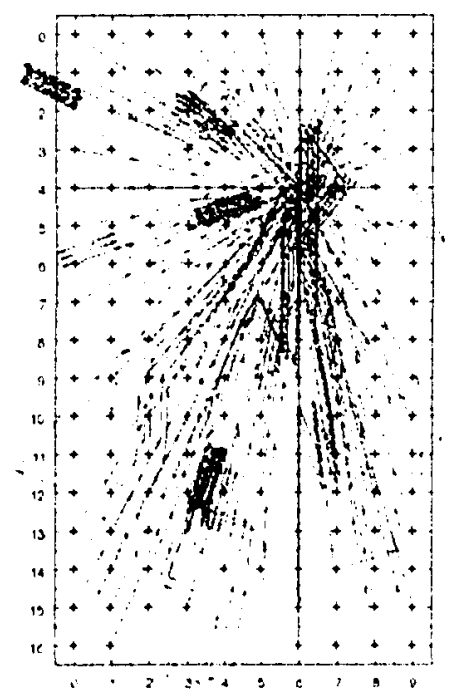

(a) $l_{\mathrm{rr}}=1.66 \mathrm{~T}$

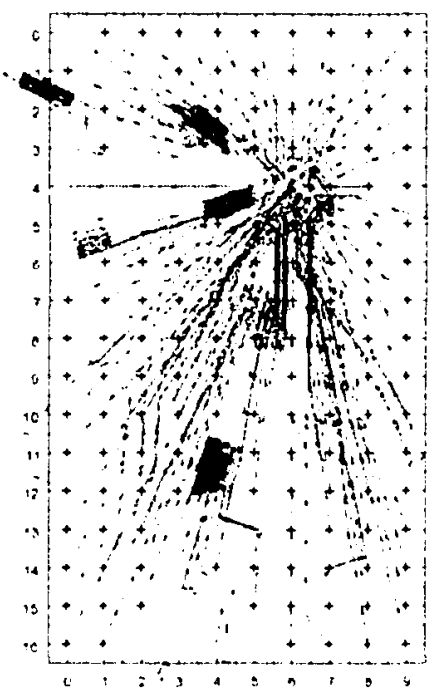

(b) $l_{\mathrm{rr}}=1.0$

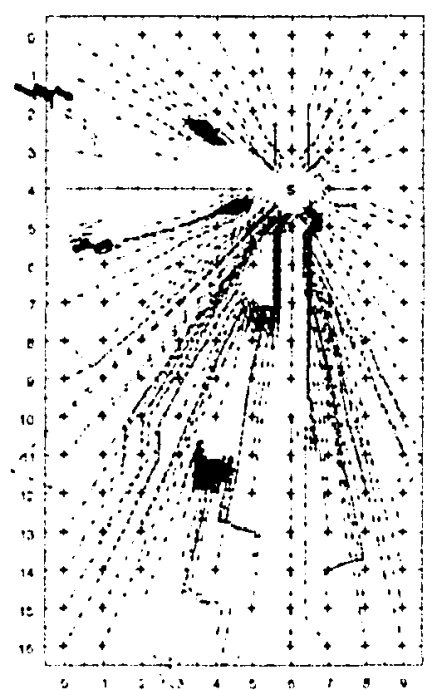

(c) $l_{\mathrm{rr}}=0.5$

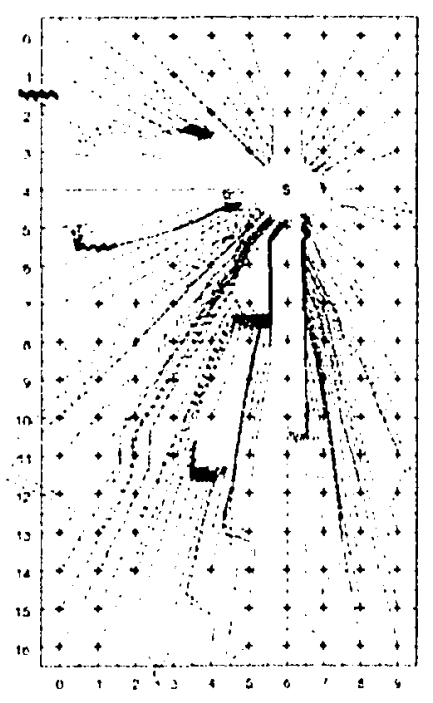

(d) $l_{\mathrm{rr}}=0.25$

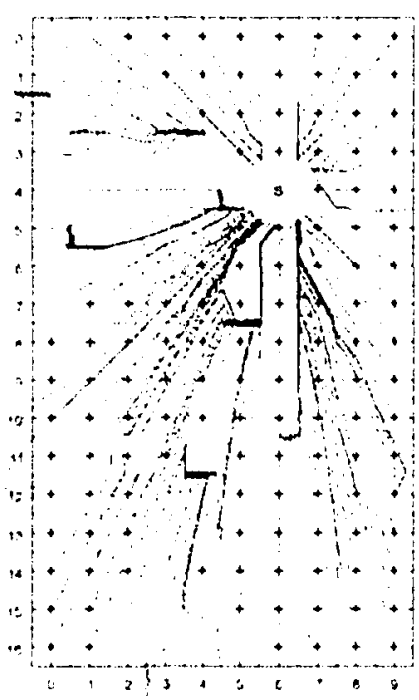

(e) $l_{\mathrm{rr}}=0.125$

Figure 3.5: Homing attempts computed for the home vectors in figure 3.t(b) with varying settings for $l_{\mathrm{rr}}$. Successful homing is indicated at the start position by a ' + symbol. 


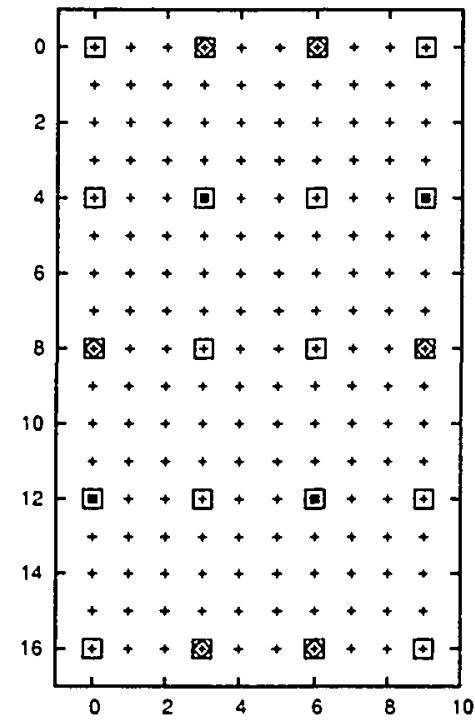

Figure 3.6: Positions of images, snapshots, robot snapshot positions, and robot start positions within the office enviromment capture grid.

\begin{tabular}{|l|l|l|}
\hline & $A A E_{\star}$ & $R R_{+}$ \\
\hline Ideal & 0 & 1 \\
\hline Impulse & 0.165 & 0.889 \\
\hline Gauss & 0.255 & 0.986 \\
\hline
\end{tabular}

Table 3.1: Values of averaged performance metrics for the demonstration methods.

cases where the snapshot images come from one image collection, yet the current image comes from another. For example, if snapshot images come from arboreal, and current images come from chairs then we would indicate the average $A A E$ in this situation as $A A E_{*}$ (arboreal, chairs).

Table 3.1 shows the values obtained for $A A E_{*}$ and $R R_{*}$ on our three demonstration methods. These metrics were averaged over 20 snapshot positions.

We again see the same pattern in the averaged metrics. Gauss does worse according to angular error, but better according to return ratio. 


\subsubsection{Statistical Tests}

The differences in the averaged metrics might be due to chance. To analyze the probability of such an occurrence statistical tests must be performed ${ }^{3}$. We assume that the distribution of the $A A E$ metric is well approximated by a normal distribution. The Kolmogorov-Smirnov test lends support for this assumption for a number of the homing methods presented in this thesis. Therefore we can use a paired t-test to test the difference in $A A E$ between two homing methods [15]. The subjects of this test are the average $A A E$ values from individual snapshot positions. As the same snapshot positions are used for all homing methods, a paired test is warranted. Conducting this test for the demonstration methods we find that Impulse has a significantly lower $A A E$ than Gauss $(\mathrm{P}<0.0001)$.

The distribution of the $R R$ metric, on the other hand, is quite different from a normal distribution. The maximum value of $R R$ is one and a number of homing methods presented in this thesis exhibit this maximum value for some snapshot positions. Therefore to test the difference in $R R$ between two homing methods the non-parametric Wilcoxon matched pairs test is used [107]. Carrying out this test for the demonstration methods we find that Gauss has a significantly higher average value of $R R$ than Impulse ( $\mathrm{P}=0.0012)$. Thus, both of the differences observed in table 3.1 are statistically significant.

Just two homing methods have been compared above. However, in later chapters we will compare differences among several homing methods. For the $A A E$ metric a repeated measures ANOVA test will be used to compare three or more homing methods. The individual subjects of the test are the $A A E$ values for each snapshot position. As the sime subjects (snapshot positions) are used for all treatments (homing methods), a repeated meisures design is required. If the null hypothesis (equal $A A E$ across methods) is rejected then the Tukey-Kramer multiple comparisons test is carried out to determine the significance of differences between methods. For the $R R$ metric Friedman's test is used. This test is essentially the non-parametric equivalent of the repeated measures ANOVA. Dunn's posttest is used to determine the significance of differences between methods should the null hypothesis (equal $R R$ across methods) be rejected [107]. All statistical tests were carried out using GraphPad's InStat packiage ${ }^{4}$.

It should be noted that the author is aware of no other use of statistical analysis to compare the performance of methods for robot visual homing. While many authors perform a careful analysis of their own algorithms, the only comparisons made between methods

\footnotetext{
${ }^{3}$ The statistical tests described in this chapter were initially proposed by Sonja Bisch-Knaden after analyzing the data presented in [98].

${ }^{-}$See http://www.graphpad.com.
} 
involve Franz et al.'s [26] warping method (see section 1.3.2) and none of these comparisons perform tests for the statistical significance of observed differences.

\subsection{Robot Homing Trials}

For some of the homing methods presented in this thesis, sobot homing trials were conducted. The purpose of these trials was to provide a check on the validity of results on the image database. Generally; good agreement was found between image database results and robot homing trial results. Practical considerations and limited availability of the robot prevented robot homing trials from being rum for all methods.

In robot homing trials the computed home vector directs the movement of the Pioneer robot. A subsampling of positions on the capture grid was used for the snapshot and start positions. Figure 3.6 shows both the four snapshot positions tested, and the six positions from which homing trials were started.

As stated in section 3.3.1, we assume the existence of a compass to allow the orientation held at the snapshot position to be recovered. The Pioneer robot was equipped with a magnetic compass-however this compass was found to be umreliable within the office enviromment. This umreliability was due, perhaps, to metal cladding in the walls or other magnetic disturbances. Therefore, the robot's built-in odometry system was used as a compass.

The robot's odometry system is based upon wheel encoders which integrate turns of the robot's two drive wheels to provide an estimate of position and heading with respect to the position where the system was last reset. The robot's integration of wheel turns is equivalent to the path integration systems of insects described in chapter 1 . Thus, just as insect path integration systems are subject to cumulative error and slippage, so is the robot's odometry compass. Also, the odometry compass can be rendered quite useless if the robot is lifted from the ground and re-positioned. Special care was taken to ensure that the odometry compass maintained low error. When positioning the robot to capture a snapshot image the robot was manually oriented by aligning the mounted laser pointers with tape marks along the wall. At the start of each homing attempt the robot was manually oriented in the same fashion and the odometry compass reset.

Whether magnetic- or odometry-based, the job of the compass is to obtain an estimate of the angular deviation between the snapshot position and the current position. The current view is then rotated by this angle and the computed home vector likewise rotated by the negative of this angle. 
All image processing and other operations were carried out on the robot's onboard Pentium III processor. The robot would process the current image, compute a home vector and then rotate to align itself with this vector. It would then travel $50 \mathrm{~cm}$ in a straight line, stop, and then capture another image. The robot was allowed 15 such moves to reach home. This is equivalent to a total travel distance of $7.5 \mathrm{~m}$ (for the return ratio performance metric on the image database, the total distance that the agent is allowed to travel is also $7.5 \mathrm{~m}$ ). The Pioneer was also equipped with a ring of sonar sensors which were used to test for imminent collision. If the robot approached within a threshold distance of any object it would halt and the homing attempt would end.

Each time the Pioneer captured an image, a separate observation camera, positioned above the Pioneer and looking down on it, would capture an image of the Pioneer. After all homing trials were complete, the position of the Pioneer was tracked by manually selecting it in images from the observation camera. The homography matrix relating the image coordinates of the Pioneer with the coordinate system used for the image database was then calculated using the normalized direct linear transformation algorithm [39] as implemented by Kovesi ${ }^{5}$.

Robot homing trials appear in chapters 6 and 8 .

\footnotetext{
SSee http://www.csse. uwa. edu.au/ pk/Research/MatlabFns/.
} 


\section{Chapter 4}

\section{Other Methods}

\subsection{Introduction}

This chapter describes three homing methods developed by other authors which satisfy one or more of the criteria stated in chapter 1 . As the number of approaches that have been proposed for visual homing is quite large (see table 1.1), it is not possible to implement and test all existing approaches. Indeed, some existing approaches such as those of Stürzl and Mallot [95] and Gourichon et al. [29] would be clifficult to re-implement as they rely on customized hardware. The three presented here have in common that they have exhibited good performance on real-world images, and that their authors have made a reasonable case for their possible implementation in the brain of an insect. It also happens that none of them use the feature-based approach favoured in this thesis, but that all utilize properties of the whole image for homing.

We present in turn Franz et al.'s warping method, Hafner's centre-of-mass ALV method, and Zeil et al.'s gradient descent method. After each method is presented its performance on the image database is also evaluated and analyzed. At the end of the chapter a statistical comparison between methods is presented and the case is made to consider only the warping method for subsequent comparisons in this thesis.

\subsection{Franz et al., 1998: Warping}

Franz et al. presented and analyzed a scene-based homing algorithm in [26]. This algorithm, which we will refer to as Warping, is scene-based in that the whole scene is involved in computing a home vector. It operates by a search process that compares warped versions 
of the current image with the snapshot image. The warping of current images is done as if the vantage point of the warped image were the snapshot position. The vantage point of the warped image is a putative snapshot position, $\mathbf{s}^{\prime}$. Parameters specifying the warp also specify the putative movement from $s^{\prime}$ to $c$. One can consider each set of parameters a guess which specifies the location of $\mathrm{s}^{\prime}$, with the warped image being a prediction of what the snapshot image would look like based upon that guess. Warping proceeds by searching through the parameter space for the warped image that best matches the stored snapshot image. The search process is tractable because the images are one-dimensional.

Warping is interesting from the perspective outlined above because of its well-reported performance characteristics and because the authors make a reasonable case for its biological plausibility. In addition to the original authors, Möller has commented positively regarding the performance and robustness of the warping method on real-world images. It is one of the most highly cited methods for visual homing and various authors have used it for their own comparative studies $[104,74,95,99,100]$.

\subsubsection{Equal Distance Assumption}

Franz et al.'s approach is based on the equal distance assumption. Under this assumption, all landmarks are presumed to be at an identical distance from s. Clearly this is a strong assumption that will be violated in most situations. However, Franz et al. show that the error due to the equal distance assumption (where it does not hold) decreases with distance to the goal under the additional assumption that the agent is in the open space around the goal. This additional assumption can be expressed as $b<d_{\mathbf{s}}$, meaning that the agent is already closer to the snapshot position than the nearest landmark.

\subsubsection{Parameterized Flow Fields}

Chapter 2's clevelopment of spherical flow fields in one dimension is enormously useful here'. Recall from section 2.2 that $\theta$ represents the image position of a landmark 1 at the snapshot position $\mathrm{s}$. At the current position, $\mathrm{c}$, the image of 1 has shifted to $\theta+\delta$. The movement from $\mathrm{s}$ to $\mathrm{c}$ is characterized by the movement angle $\alpha$, the distance of movement $b$, and the orientation change $\psi$. Figure 2.1(a) depicts these angles.

We pick up the development in section 2.2 at equation (2.4) which is repeated below:

$$
\tan (\psi+\delta)=\frac{b \sin (\theta-a)}{d_{s}-b \cos (\theta-a)}
$$

\footnotetext{
${ }^{1}$ Indeed, that development follows Franz et al.
} 
Franz et al. transform the above equation into a format where the equal distance assumption can be used to reduce the number of free parameters. This development is followed here. First, we stipulate a typical landmark distance $R$. The deviation of a landmark from this typical distance is expressed as $r$. Thus, the distance of the landmark from $\mathbf{s}$ is $d_{\mathbf{s}}=R+r$. Substituting this into the equation we obtain

$$
\begin{aligned}
& \tan (\psi+\delta)=\frac{b \sin (\theta-a)}{R+r-b \cos (\theta-\alpha)} \\
& \tan (\psi+\delta)=\frac{\frac{b}{R} \sin (\theta-\alpha)}{1+\frac{r}{R}-\frac{b}{R} \cos (\theta-\alpha)}
\end{aligned}
$$

We now introduce a new variable $\rho$ which stands for the ratio of displacement distance to landmark distance. That is, $\rho=b / R$. Finally; we incorporate the equal distance assumption, which means that $r=0$. We now substitute in $\rho$ and set $r=0$ and then rearrange terms to obtain an expression for $\delta$ :

$$
\begin{aligned}
\tan (\psi+\delta) & =\frac{\rho \sin (\theta-\alpha)}{1-\rho \cos (\theta-a)} \\
\delta & =\arctan \left(\frac{\rho \sin (\theta-\alpha)}{1-\rho \cos (\theta-\alpha)}\right)-\psi
\end{aligned}
$$

Equation (4.5) characterizes the one-dimensional flow field $\delta(\theta)$ parameterized by $a, \rho$, and $\psi$. This flow field approximates the true flow field experienced by an agent displaced from $\mathrm{s}$ to $\mathrm{c}$, where the parameters $\alpha, \rho$, and $\psi$ describe the agent's displacement.

\subsubsection{Warping}

Let us briefly review the information available to the homing agent. Such an agent has the current image $C$ and the snapshot image $S$. It is equivalent to say that the agent 'knows' its snapshot position $s$ and wishes to determine its current position $\mathbf{c}$, or to say that it 'knows' c and wishes to determine s. Let us say that $\mathbf{c}$ is known. In this case we might make an arbitrary guess that the snapshot position is at $\mathbf{s}^{\prime}$, where the displacement between $\mathbf{c}$ and $\mathbf{s}^{\prime}$ is described by the parameters $a, \rho$, and $\psi$. To test this guess the agent could travel from $\mathbf{c}$ to $\mathbf{s}^{\prime}$ and then compare $C$ and $S$. If the two images were sufficiently similar then the agent might decide that it has arrived home. If the images were dissimilar then the agent could return to its previous position and malie another guess. Such movements are costly in terms of energy and time, particularly if many guesses are required.

The warping method operates by making and evaluating such guesses without actually moving. The putative snapshot position $\mathbf{s}^{\prime}$ is evaluated by warping the current image $C$ to 
find a warped image $S^{\prime}$, and then comparing $S^{\prime}$ to $S$. Equation (4.5) approximates the flow that would have occurred for a movement from $\mathbf{s}^{\prime}$ to $\mathbf{c}$ specified by parameters $\alpha, \rho$, and $\psi$. Thus the mapping between pixels in $C$ to pixels in $S^{\prime}$ is as follows:

$$
S^{\prime}(\theta)=C(\theta+\delta)
$$

Iterating the above equation for all $\theta$ implements the warp. For each iteration $\delta$ must be computed from equation (4.5). Speedup can be obtained by pre-computing $\theta+\delta$ for the required range of $a, \rho, \psi$, and $\theta$. Indeed, this speedup is employed in the implementation used here ${ }^{2}$.

\subsubsection{Pre-Processing}

The warping method operates on one-dimensional images. To obtain such images the region around the horizon of a two-dimensional panoramic image is extracted. This region is defined by a fixed angular distance above and below the horizon. The conversion to a onedimensional image occurs through summing along each vertical column within the extracted region. Each sum is entered as a pixel at the corresponding position of the one-dimensional image. This one-dimensional image may then be low-pass filtered and downsampled by a factor, $m_{x}$. Figure 4.1 shows a two-dimensional image and the low-pass filtered one-dimensional image obtained from it. The low-pass filter used here and elsewhere in this thesis is implemented by convolution with the following Gaussian kernel:

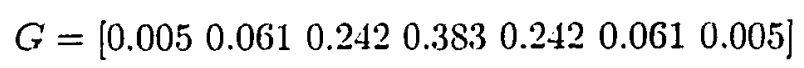

applied separately in the $x$ and $y$ directions.

The minimum practical length for a Gaussian kernel is five. A smaller kernel cannot adequately represent the Gaussian distribution (see [96], chapter 3). Thus the kernel $G$ is just slightly larger than the smallest possible Guassian kernel. The value of $\sigma$ for $G$ is approximately one. Therefore the value of $\sigma^{\prime}$ for the same kernel in the frequency domain is also approximately one $\left(\sigma^{\prime}=1 / \sigma\right)$. A broader Gaussian in the spatial domain corresponds to a narrower Gaussian in the frequency domain, which increases the clegree of low-pass filtering. Repeated convolution of a signal with a Gaussian kernel is equivalent to convolving the same signal with another Gaussian kernel that has a larger $\sigma$. Thus, repeated convolution with $G$ provides a convenient building block for constructing low-pass filters with smaller cut-off frequencies (filters with smaller cut-off frequencies cause a stronger blurring effect).

\footnotetext{
${ }^{2}$ The implementation of Warping used here is due to Ralf Möller.
} 

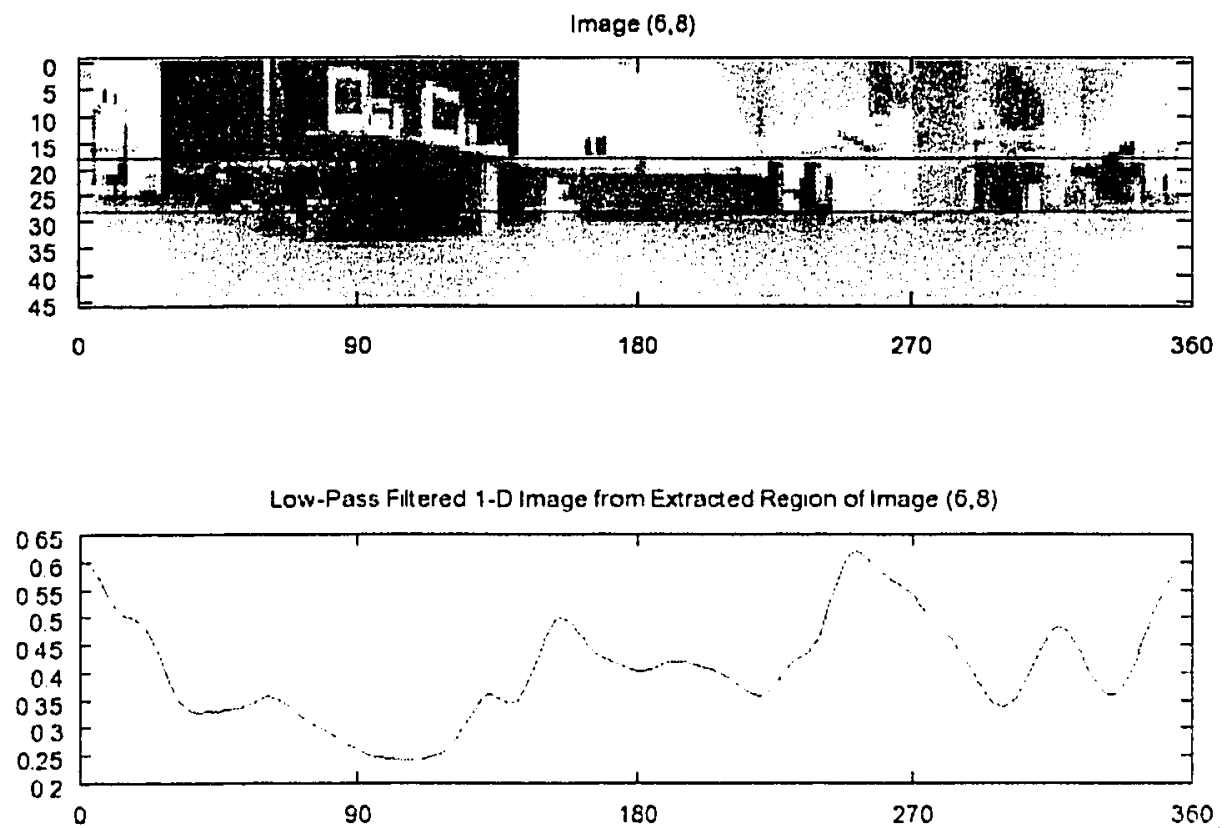

Figure 4.1: On the top is image $(6,8)$ from image collection original. This image is converted to the one-dimensional image on bottom through the process described in the text. The bars in the top image delimit the extracted region which extends $5^{\circ}$ above and below the horizon. The image is then low-pass filtered through 10 applications of the Gaussian filter. The $\mathrm{x}$-coordinates of both plots are in clegrees, not image indices.

\subsubsection{Tested Algorithm}

A search is carried out over the parameters $a, \rho$, and $\psi$. For each tested combination of these parameters the current image $C$ is warped to $S^{\prime}$ according to equations (4.5) and (4.6). $S^{\prime}$ is then compared with the stored snapshot image $S$. Two different means for comparing these images are tested. Franz et al. compare two one-dimensional images by finding their dot product. The other possibility is to find the sum of squared differences between the two images. The parameters generating the warped image most similar to $S$ form the outputs of the search process. The home vector is computed from these parameters as follows:

$$
\mathbf{v}_{\text {warp }}=\left(\begin{array}{c}
\cos (\alpha-\psi+\pi) \\
\sin (\alpha-\psi+\pi)
\end{array}\right)
$$

The parameter $\rho$ can optionally be used to scale the home vector so that the homing agent malies larger steps when far from the goal, and smaller steps when nearby. 
The configuration parameters for Warping specify the search density for each of the three motion parameters. Parameters $n_{\alpha}$, and $n_{\psi^{\prime}}$ give the number of search steps for $\alpha$ and $\psi$, respectively; in the range $[0,2 \pi]$. Parameter $n_{\rho}$ gives the number of search steps for $\rho$ in the range $[0,1-\epsilon]$, where $\epsilon$ is a small constant (0.05) that prevents searching the value $\rho=1$ which would lead to division by zero. If $n_{\imath}$ ' is set to ' 1 ' it implies that no search is done in that dimension and $\psi$ will be set to zero. This setting is acceptable for these experiments because the snapshot and current images are always assumed to be taken from the same orientation.

Figure 4.2 illustrates a very small scale search with $n_{a}=4$ and $n_{\imath},=1$. No search is done over $\rho$ which is fixed at 0.5 . The current image $C$ is warped according to the four values of $\alpha$. Each $a$ value corresponds to a putative snapshot position. The warped image at the 'northern' position $\mathbf{n}$ is most similar to the snapshot image $S$ captured at the true goal position s.

Franz et al. test Warping using a robot in an arena populated with toy houses. This environment appears to be relatively sparse in detail. They report good results when the robot is in the open space around the goal position. However, performance goes down when the goal is too near to one of the toy houses.

\subsubsection{Optimizing Parameters}

The approach taken throughout this thesis for all homing methods is to optimize performance on the original image collection by doing a search for best parameters ${ }^{3}$ on that collection. Each combination of parameters is judged by configuring the homing method with those parameters and then determining $A . A E_{*}$ (original). The combination with the lowest value is then used for subsequent experiments. Thus, original is the 'home ground' for all methods.

The parameters tested for Warping are:

- Angular distance above and below the horizon defining the region of the two-dimensional image used to create the one-dimensional image: $5^{\circ}, 10^{\circ}$, or the whole image $\left(23^{\circ}\right)$.

- Downsampling factor for one-dimensional images, $m_{x}: 1$ (no downsampling), 2, or 4.

- Number of applications of the Gaussian low-pass filter: $0,1,3$ or 5 .

\footnotetext{
${ }^{3}$ The term 'parameters' here refers to the homing method's configuration parameters, not to the motion parameters $\alpha, \rho$, and $\psi$ ' discussed above.
} 


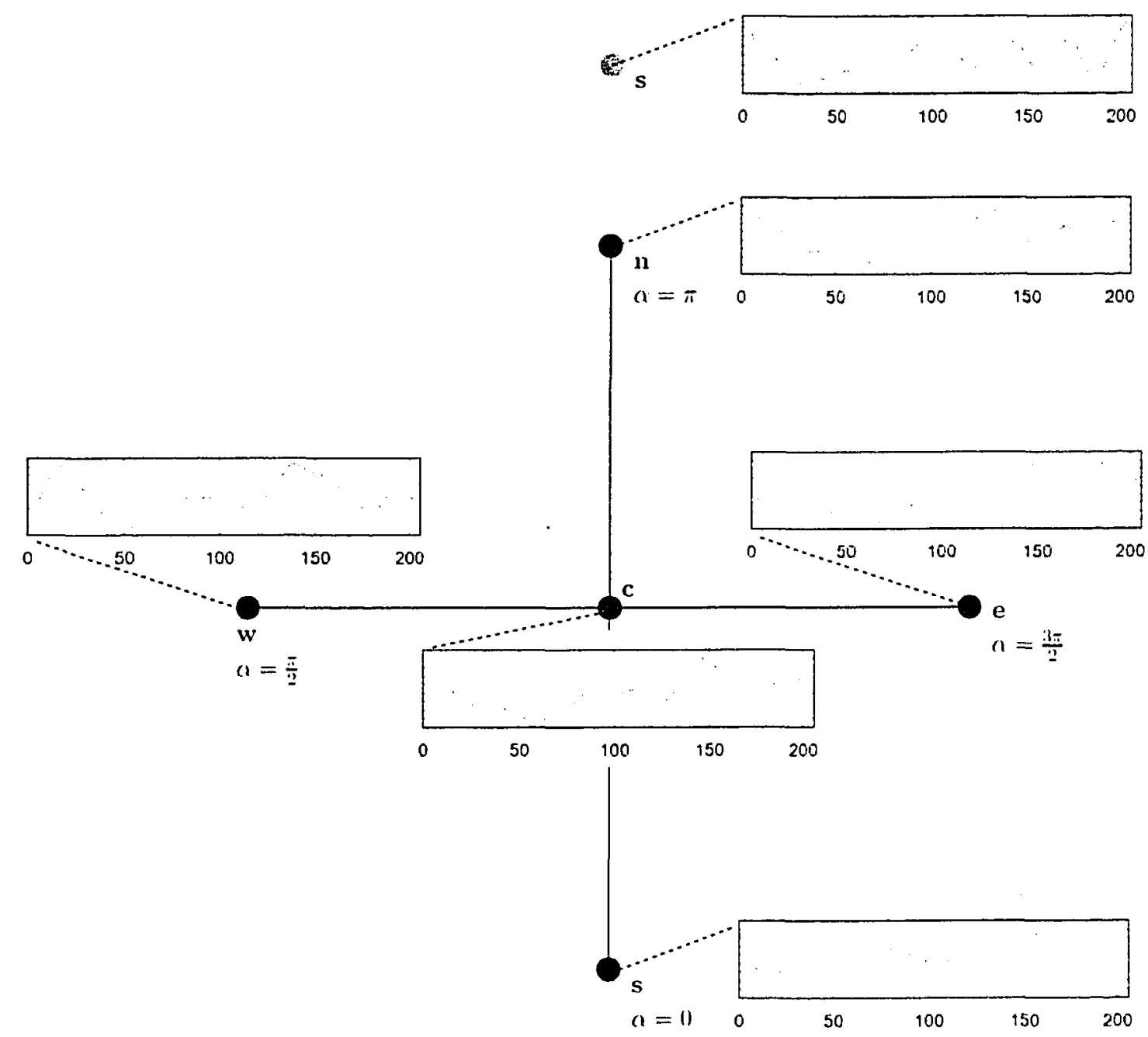

Figure 4.2: Demonstration of Warping with a small search space. The current position $\mathbf{c}$ is at the centre and four putative snapshot positions (n, e, s, w) are in the four cardinal directions. The true snapshot position is at $\mathbf{s}$, marked by a grer point. The current one-dimensional image $C$ is shown adjacent to $\mathrm{c}$ and warped versions of this image are shown adjacent to the four putative snapshot positions. 
- Number of search increments for $\alpha, n_{a}: 32$ or 64 .

- Number of search increments for $\rho, n_{\rho}: 32$ or 64 .

- Number of search increments for $\psi, n_{\psi}: 1,32$ or 64 .

- Method of comparison between warped and snapshot images: sum of squared differences or dot product.

The best combination found for Warping is indicated in bold above. Generally, the performance of this method is relatively robust to most of these parameters (as mentioned in [74]). Interestingly, the best combination has $n_{v^{\prime}}=1$ meaning that no search is done for rotation. This makes sense as the inages in the database were all taken from the same orientation. The mere fact that the best combination has $n_{\mathrm{t}^{\prime}}=1$ means that adding rotation as an extra dimension of search can impair performance.

\subsubsection{Results on Image Database}

\section{Image Collection original}

Figure 4.3 shows home vectors generated by Warping on image collection original for snapshot positions $(6,4)$ and $(0,16)^{4}$. The figure captions give the computed values of $A . A E$ and $R R$ for these snapshot positions. For snapshot position $(6,4)$ Warping exhibits a near perfect home vector field. The high quality of this vector field is reflected in the very low value for $A A E_{(6,4)}$, and the maximum value of unity for $R R_{(6,4)}$. However, for snapshot position $(0,16)$ the vector field is significantly degraded. Correct vectors are found for only a small fraction of the vector field around $(0,16)$. The value of $A A E_{(0,16)}$ is quite large (greater than $\frac{\pi}{2}$ ) and the value of $R R(0.266)$ indicates that the catchment area covers only about a quarter of the capture grid.

Clearly then, the performance of Warping is dependent on the location of the snapshot position. In order to asses this dependence the values of $A A E$ and $R R$ are plotted for all of the 20 snapshot positions depicted in figure 3.6. Figure 4.4 present these plots. The poor performance of Warping at snapshot position $(0,16)$ is clearly reflected in both $A A E$ and $R R$. Generally, it performs poorly along the boundaries of the capture grid and particularly in the corners (the corner at $(9,16)$ appears to be an exception). The results for $A A E$ and $R R$ are in rough agreement, in that the same trend can be observed for both metrics.

\footnotetext{
${ }^{4}$ Positions $(6,4)$ and $(0,16)$ are used for demonstration of home vectors throughout this thesis. Position $(6,4)$ is relatively central and most methods appear to perform well here (if at all). Position $(0,16)$ appears to be one of the most difficult positions for most methods.
} 


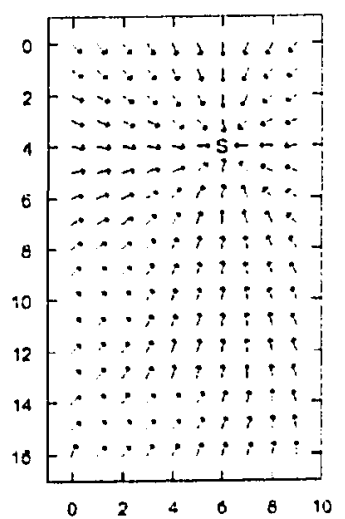

(a) Warping, $\mathbf{s}=(6,4)$ $A . A E=0.119$, $R R=1.000$

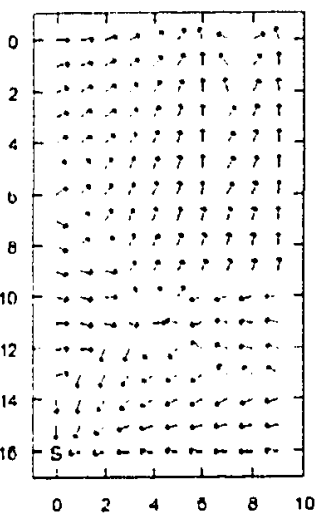

(b) Warping, $\mathbf{s}=(0,16)$ : $A A E=1.758$, $R R=0.266$

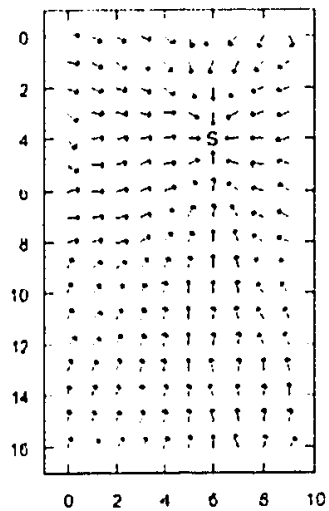

(e) GradDescent, $\mathrm{s}=(6,4)$ : $A . A E=0.225$, $R R=1.000$

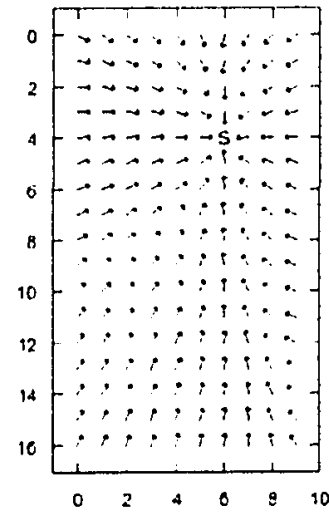

(c) COMALV, $\mathrm{s}=(6,4)$ : $A . A E=0.199$, $R R=1.000$

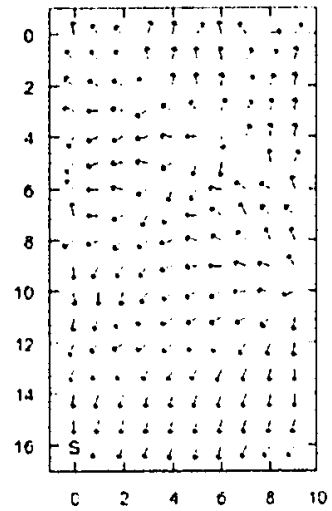

(f) GradDescent, $s=(0,16):$ $A A E=1.310$, $R R=0.331$

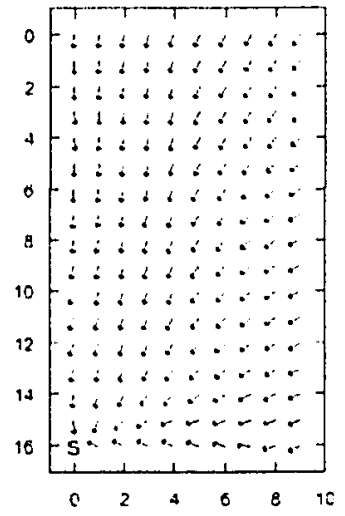

(d) COMALV, $\mathrm{s}=(0,16)$ : $A A E=0.12 \bar{i}$ $R R=0.888$

Figure 4.3: Home vector fields for Warping (a,b), COMALV (c,d), and GradDescent $(e, f)$, for snapshot positions $(6,4)(a, c, e)$ and $(0,16)(b, d, f)$ in image collection original. The snapshot position is indicated with a red ' $s$ '. 
It makes sense that Warping should perform poorly along the boundaries and corners of the capture grid. The boundaries of the capture grid are adjacent to the walls, desks, and chairs of the office environment. Thus, the equal distance assumption will be violated most strongly at snapshot positions near these objects. Still, Franz et al. argued that even when the equal distance assumption is violated, the error remains small as long as $b<d_{\mathbf{s}}$. Yet if we are at the boundary then $d_{\mathbf{s}}$ will be small. Thus, the inequality will be satisfied only for very small displacements from $s$.

Also, the region at the bottom of the capture grid is problematic for another reason. At the end of the room which this region represents there is a large bench against a white wall. Images taken here show the bench as large and dark. From further out, however, the white wall is more prevalent. This may also contribute to Warping's poorer performance in the bottom left corner of the capture grid.

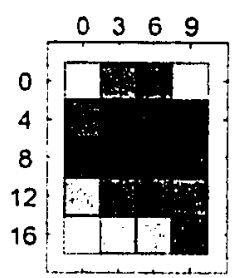

(a) Warping: $A A E_{*}=$ $0.576 \pm 0.461$

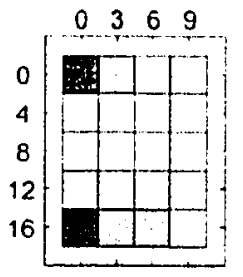

(d) Warping: $R R_{*}=$ $0.792 \pm 0.226$

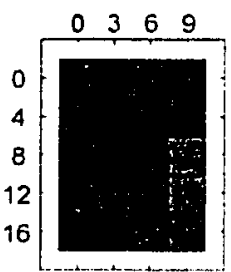

(b) COMALV:

$A A E_{*}=$

$0.252 \pm 0.0982$

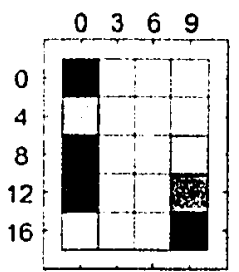

(e) COMALV: $R R_{\text {, }}=$ $0.731 \pm 0.398$

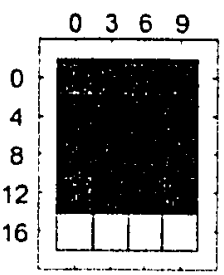

(c) GradDescent: $A A E_{*}=$ $0.546 \pm 0.383$

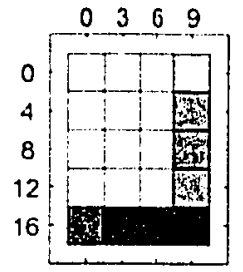

(f) GradDescent: $R R_{+}=$ $0.731 \pm 0.329$

Figure 4.4: $A A E$ and $R R$ across snapshot positions for all methods on image collection original. Beneath each plot is shown the mean value of the metric $\left(A A E_{*}\right.$ or $R R_{*}$ ) and its standard deviation (the number following the ' \pm ' symbol). Average values for $A A E \max .=1.758, R R$ max. $=1$. 


\section{Image Collections hall1 and hal12}

Figure 4.5 shows home vectors generated by Warping on image collections hall1 and hall2 for snapshot positions $(3,12)^{5}$. The home vector fields shown for Warping are both almost entirely correct, however the vectors in hall2 are slightly worse (which is reflected in the computed $A A E$ and $R R$ values).

Figure 4.7 shows how $A A E$ and $R R$ vary across snapshot positions. $A . A E$ is rather constant throughout, however some large deviations in $R R$ are apparent. As an example, consider position $(3,4)$, the home vector field for which is shown in figure 4.6 (a). To use the terminology of complex systems, the attractor of the home vector field is not coincident with snapshot position $(3,4)$. This problematic situation is not reflected in the value for $A A E$ $(0.488)$ which is still reasonably low. However, the very low $R R$ value $(0.106)$ appropriately indicates that the quality of homing is poor. This example shows the wisdom of retaining two performance metrics.

Figure 4.8 show $A A E$ and $R R$ for Warping in image collection hall2. The performance of Warping is generally good in hall2 with slight degradation near the bottom portion of the capture grid (most apparent in $R R$ ).

\section{Cross-Collection Test}

In addition to original, seven other image collections were captured in the office enviromment. These allow us to determine not only how a method such as Warping performs within a single environment, but how it performs when snapshot images are taken from one environment and current images taken from another. In this way; we can determine the robustness of performance to a changing environment.

For the eight office enviromment image collections, there are 64 pairings. Each pairing involves taking twenty snapshot images from the first collection and allowing the method in question to compute home vectors for all images from the second collection. We compute the $A A E_{\mathbf{s}}(\mathrm{a}, \mathrm{b})$ then for each snapshot position $\mathrm{s}$, and average these over all twenty snapshots to obtain $A A E_{*}(\mathrm{a}, \mathrm{b})$, where $\mathrm{a}$ is the collection from which snapshot images are drawn, and $\mathrm{b}$ is the collection from which current images are drawn. Figure 4.4 presented the result of pairing image collection original with itself. Figure 4.9 plots $A A E_{*}$ and $R R_{*}$ for all pairings. To obtain an overall measure of homing performance we average $A A E_{*}$ over all pairings to obtain the cross-collection angular crror $A . A E_{*}(*, *)$. The average of $R R_{*}$ over all pairings yields the cross-collection return ratio $R R_{*}(*, *)$.

\footnotetext{
${ }^{5}$ Position $(3,12)$ is used for demonstration of home vectors on both hall1 and hall2 throughout this thesis.
} 

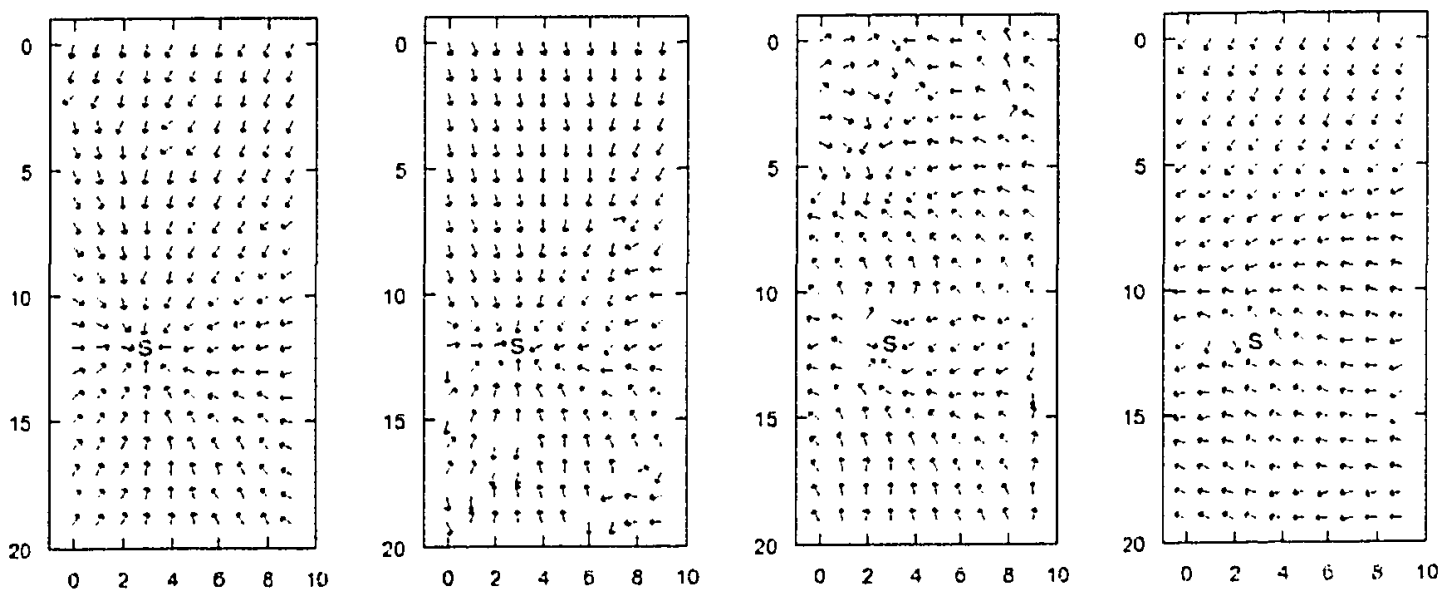

(a) Warping,

hall1,

(b) Warping,

hall2,

(c) COMALV,

hall1,

(d) COMALV,

$\mathbf{s}=(3,12)$ :

$A A E=0.40 \overline{\%}$,

$\mathrm{s}=(3,12)$ :

$A A E=1.168$,

$A A E=0.160$,

$R R=1.000$

$R R=0.915$

$R R=0.286$

hall2,

$\mathbf{s}=(3,12):$

$A A E=0.93 \bar{\top}$,

$R R=0.060$
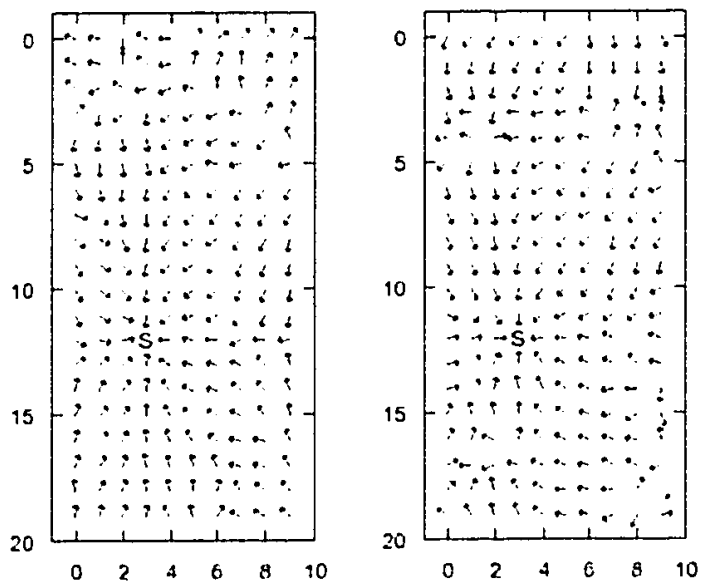

(e) GradDescent, hall1,

(f) GradDescent, hal12, $\mathbf{s}=(3,12)$ : $A A E=0.711$, $\mathrm{s}=(3,12)$ : $A . A E=0.661$, $R R=0.824$ $R R=0.7 T 9$

Figure 4.5: Home vector fields for Warping (a,b), COMALV (c,d), and GradDescent $(\mathrm{e}, \mathrm{f})$, for snapshot position $(3,12)$ in image collections hall1 and hall2. 


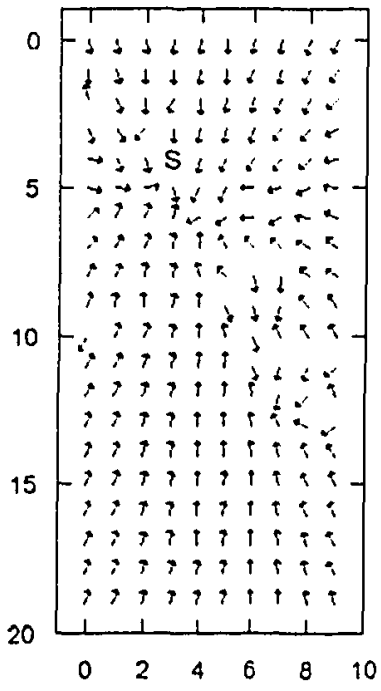

(a) Warping, hall $1, s=(3,4)$ : $A A E=0.488, R R=0.106$

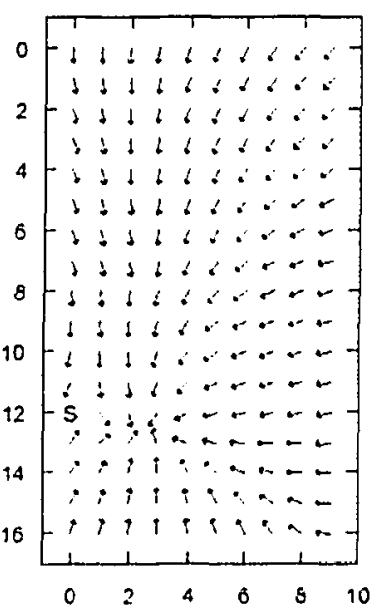

(b) COMALV, original, $\mathrm{s}=(0,12)$ : $A . A E=0.316, R R=0.047$

Figure 4.6: Home vector fields for Warping and COMALV that warrant special attention in the text.

The performance of Warping remains relatively robust to many changes. To assess the impact of changes due to the introduction and remoral of objects, the relevant image collections are: original, chairs, arboreal, and screen. Values of $A A E_{*}$ and $R R_{*}$ for all possible combinations of these collections can be found in $4 \times 4$ blocks in the upper left of the plots in figure 4.9. The variation of both $A . A E_{*}$ and $R R$, within these blocks appears relatively low.

To assess the impact of changes in illumination the relevant image collections are: original, day, twilight, doorlit, and winlit. Values for all of the possible combinations between these collections (excluding original) appear in $4 \times 4$ blocks in the lower right of the same plots. Combinations including original are shown in parts of the top row and the left-most column. Overall, the variation for illumination changes is greater. In particular the worst combinations for Warping occur if either doorlit or winlit is used as the snapshot image collection, and any other collection is used for current images. This is due to the very large change in illumination caused by turning off one of the light bars. Interestingly, when winlit is paired with winlit, warping exhibits its lowest $A A E_{*}(0.219)$ and its highest $R R_{*}(0.939)$. A room that is half in light and half in shadow can be easily mapped to one-dimensional images that are quite useful for navigation. 


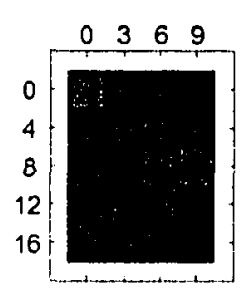

(a) Warping: $A A E_{*}=$ $0.295 \pm 0.151$

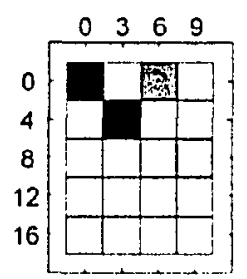

(d) Warping:

$R R_{*}=$ $0.8+1 \pm 0.289$

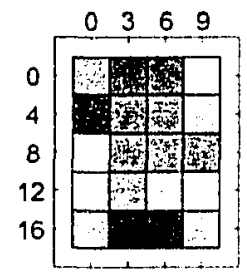

(b) COMALV:

$$
A A E_{*}=
$$$$
1.21 \pm 0.411
$$

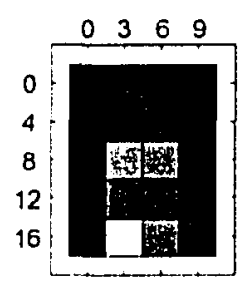

(e) COMALV:

$$
R R_{+}=
$$$$
0.141 \pm 0.204
$$

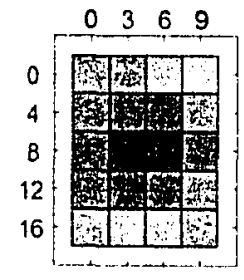

(c) GradDescent:

$$
A A E \text {. }=
$$

$0.919 \pm 0.232$

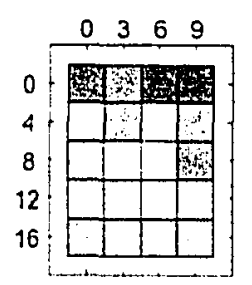

(f) GradDescent:

$R R_{+}=$

$0.608 \pm 0.169$

Figure 4.7: $A A E$ and $R R$ across snapshot positions for all methods on image collection hall1. $A A E \max$. $=2.288, R R \max .=1$.

\section{Robot Homing Trials}

Robot homing trials were conducted for Warping. However, in order to present these trials in context they do not appear until chapter 6 where they can be contrasted with trials rum on one of the methods developed in this thesis (robot homing trials were not executed for either of the two remaining methods presented in this chapter).

\subsection{Hafner, 2001: Centre-of-Mass ALV (COMALV)}

We now review the homing method described by Hafner in [33]. The development of this homing method is unusual in that the method itself was learned by a neural network. The difference image of two one-dimensional images was fed as input into a network which was trained to output correct vectors directed from the position of the first image to the position of the second image. This 2-layer network was trained through Hebbian learning with the correct home vectors used as the target outputs. The network's output layer encodes the $x$ and $y$ components of the home vector. The one-dimensional difference image is fed into the 


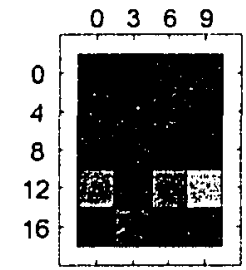

(a) Warping:

$$
A . A E_{*}=
$$

$0.457 \pm 0.185$

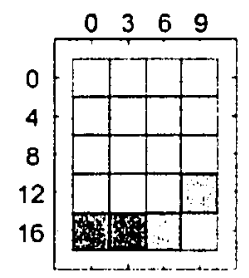

(d) Warping:

$R R,=$

$0.798 \pm 0.202$

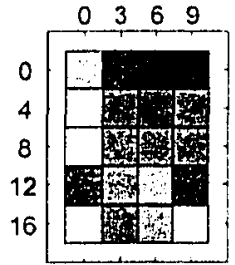

(b) COMALV:

$$
A A E_{*}=
$$$$
0.941 \pm 0.372
$$

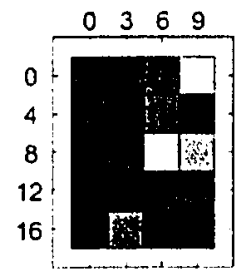

(e) COMALV: $R R_{*}=$

$0.171 \pm 0.213$

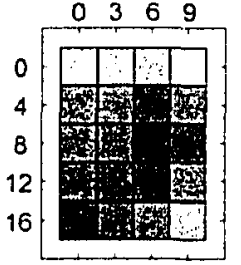

(c) GradDescent: $A A E_{*}=$ $0.872 \pm 0.254$

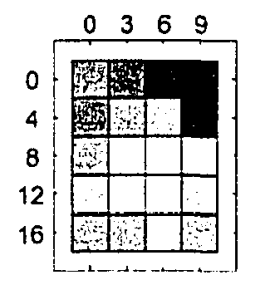

(f) GradDescent: $R R_{\star}=$ $0.53 \bar{\top} \pm 0.228$

Figure 4.8: $A A E$ and $R R$ across snapshot positions for all methods on image collection hal12. $A A E$ max. $=2.127, R R$ max. $=1$.

input layer which has weighted connections to the output layer. In a previous approach by the same author two other neural network architectures were used for the same task [35].

According to Hafner's analysis, the method converged upon by all of her networks is functionally equivalent to the ALV model with the average landmark vector being directed towards the image's centre-of-mass (the ALV model was reviewed in chapter 1). We will refer to this method as the Centre-Of-Mass ALV method, or COMALV. Aside from COMALV's evolution through a neural learning process, it possesses several other interesting characteristics. Hafner showed that it could generate correct home vectors on real-world images from at least one test environment. Also, it is an extraordinarily simple method with very low computational complexity: These characteristics make it suitable for presentation here.

This section will describe COMALV without further reference to its particular implementation in Hafner's networks. The implementation of COMALV used here is based only on the description provided below. 


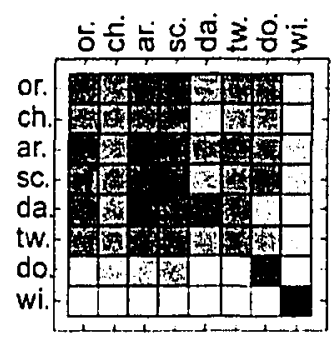

(a) Warping:

$A A E *(*, *)=$ $0.836 \pm 0.361$

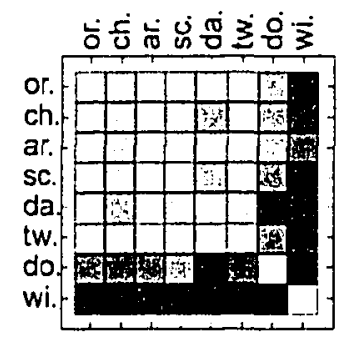

(d) Warping:

$R R_{*}(*, *)=$

$0.551 \pm 0.251$

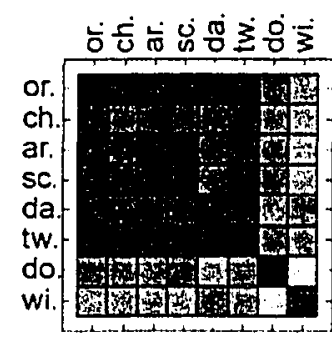

(b) COMALV:

$A A E_{*}(*, *)=$ $0.533 \pm 0.198$

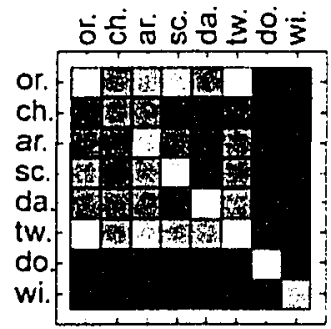

(e) COMALV:

$R R_{+}(*, *)=$ $0.291 \pm 0.218$

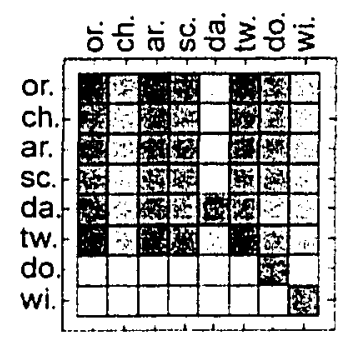

(c) GradDescent: $A A E_{*}(*, *)=$ $0.945 \pm 0.34$

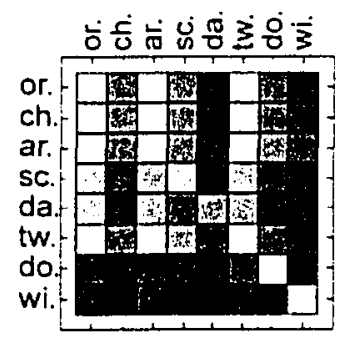

(f) GradDescent: $R R .(*, *)=$ $0.37 \& \pm 0.211$

Figure 4.9: $A A E_{*}$ and $R R_{*}$ over all pairings of the eight office image collections. The image collection used for snapshot images is shown on the vertical axis, while the collection used for current images is shown on the horizontal axis. Beneath each plot is shown the mean value of the metric $\left(A A E_{*}(*, *)\right.$ or $\left.R R_{*}(*, *)\right)$ and its standard deviation (the number following the ' \pm ' sumbol). Abbreviations: or. = original, ch. = chairs, ar. = arboreal, sc. = screen, da. = day, tw. = twilight, do. = doorlit, wi. $=$ winlit. $A A E \max .=1.811, R R \max .=1$. 


\subsubsection{Extracting the Centre-Of-Mass}

COMALV operates on one-dimensional images extracted in the same manner as described in section 4.2.4 (with one slight exception, see section 4.3.3). For both the snapshot and current images, the average landmark vector is extracted from the one-dimensional image as follows:

$$
A L V=I(\theta)\left(\begin{array}{c}
\cos (\theta) \\
\sin (\theta)
\end{array}\right)
$$

where $I$ is the one-dimensional image and $I(\theta)$ is the value of that image at the index corresponding to $\theta$.

In the original ALV model $[57,58]$, discrete landmarks were first segmented from the background. Unit vectors directed to each of these landmarks would then be averaged together to form the $A L V$. However, in COMALV there is no segmentation of landmarks from background. Instead, the $A L V$ represents the centre-of-mass of the input image's greyscale values.

\subsubsection{Tested Algorithm}

$A L V_{\mathrm{s}}$ is extracted at the snapshot position and stored. As in the original ALV model, the snapshot image $S$ need not be stored. $A L V_{c}$ is extracted at the current position. The home vector is then simply calculated:

$$
\mathrm{v}_{\text {COMALV }}=A L V_{\mathrm{c}}-A L V_{\mathrm{s}}
$$

\subsubsection{Optimizing Parameters}

The parameters tested for COMALV are:

- Position of the two-dimensional image region used to create the one-dimensional image: below the horizon, at the horizon $\left( \pm 5^{\circ}\right)$, above the horizon, or the whole image.

- Downsampling factor for one-dimensional images, $m_{x}: 1$ (no downsampling), 2 , or 4 .

- Number of applications of the Gaussian low-pass filter: $0,1,3$ or 5 .

The best combination found is indicated in bold above. The choice of extracting onedimensional images from differing regions of the input images was prompted by some of the findings presented below. 


\subsubsection{Results on Image Database}

\section{Image Collection original}

Figure 4.3 shows home vectors generated by COMALV on image collection original for snapshot positions $(6,4)$ and $(0,16)$. For snapshot position $(6,4)$ COMALV, like Warping, exhibits a near perfect home vector field. Unlike Warping, the home vector field for snapshot position $(0,16)$ is also quite good.

Figure 4.4 presents the plots of $A A E$ and $R R$ across all twenty tested snapshot positions. The $A A E$ plot shows relatively low error. Notable in this plot is the lack of particular large peaks in error as occur for Warping. However, the $R R$ plot indicates a small catchment area for several snapshot positions. Figure 4.6(b) shows the home vector field for snapshot position $(0,12)$ where we see a similar problem as described above for Warping. That is, the vector field's point of attraction is not coincident with the snapshot position.

\section{Image Collections hall1 and hall2}

Figure 4.5 shows home vectors generated by COMALV on image collections hall1 and hall2 for snapshot position $(3,12)$. These home vector fields, in contrast to all those discussed thus far, are almost entirely incorrect. Figures 4.7 and 4.8 show similarly poor results for $A A E$ and $R R$ across all twenty snapshot positions in both hall1 and hall2 (compare with the results for Warping).

Clearly there is some property of original that allows COMALV to succeed, yet which is lacking in hall1 and hal12. This property is revealed by studying the average landmark vectors for these different enviromments. Figure 4.10 shows these average landmark vectors for original, hall1, and hall2, and for three settings of the parameter describing the region of the two-dimensional image used to generate one-dimensional images. The parameter search has set this parameter to the region above the horizon. Figure 4.10 (c) shows the average landmark vectors for original with this parameter setting. This average landmark vector field has an attractor point at about position $(6,5)$ which all vectors approximately point towards. The presence of such an attractor point is crucial to COMALV's success. For the same parameter setting, no such attractor exists for hall1 and hal12-hence the poor results reported above.

The attractor is a point in space towards which the average landmark vector, defined by equation (4.8), is directed. In a sense, the attractor is like a landmark in that the change in its position between the snapshot and current images can be used to direct the agent's homing. This is exactly what is achieved by equation (4.9). However, as the method is 
essentially relying upon a single landmark, any instability in that landmark is detrimental to homing. In figure 4.10 (f) all average landmark vectors are directed in approximately the same direction. Hence, a comparison between them yields no useful information. Figure 4.10 (i) shows that for hall2 the average landmark vectors do not agree on a single attractor point.

We can also observe that a roughly defined attractor point does exist for hall1 for the parameter setting below (see figure $4.10(\mathrm{~d})$ ). Indeed, if the parameter search from section 4.3.3 is repeated, only on hall1 as opposed to original, then the parameter setting below is part of the best configuration. If it was allowed for a homing method to customize its parameters for every new environment then COMALV could be appropriately customized for hall1. However, this would require that the agent homing by COMALV would first need to thoroughly explore an area before taking a snapshot image. This exploration would be necessary in order to find that part of the image which generated stable average landmark vector fields, with a single well-defined attractor point, throughout the environment. However, such exploration is costly in terms of time and energy:

Interestingly; it is known that insects do explore novel sites in their enviromments by performing learning flights (see section 1.2.4). Typically such exploration is thought to perform some kind of input filtering-perhaps allowing the insect to separate nearby landnarks from distant ones. Yet, it is not inconceivable that the insect is determining what parts of the image to use for centre-of-mass computations as in COMALV. This point, howerer, is quite speculative and it is considered more important here to focus on homing methods that are robust without incurring extra computational and energetic effort.

\section{Cross-Collection Test}

Figure 4.9 plots $A A E_{*}$ and $R R_{*}$ for all pairings between image collections from the office environment. In comparison to Warping, relatively little variation is found in $A-A E$. In the absence of the $R R$ metric this might lead us to believe that COMALV was quite robust to environmental modifications. However, the plot of $R R_{+}$shows considerably higher variation. As for Warping, the combinations involving doorlit and winlit are particularly problematic. Also, a band of high $R R_{*}$ values is noticeable along the plot's main diagonal. This diagonal band shows that COMALV tends to perform better when both snapshot and current images come from the same image collection. This is unsurprising, but it is notable that Warping does not exhibit such a band-indicating, perhaps, that it is more invariant to environmental changes.

Note that no numerical comparisons have as yet been made between the metrics for 


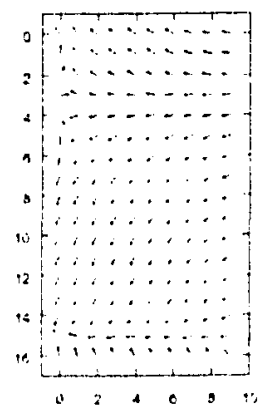

(a) original, below

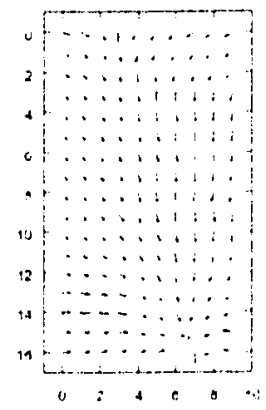

(d) hall1, below

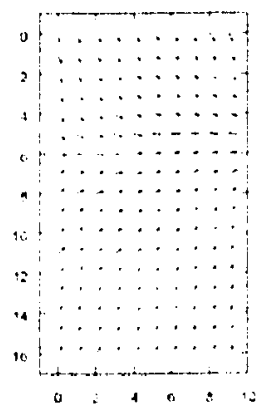

(g) hal12, below

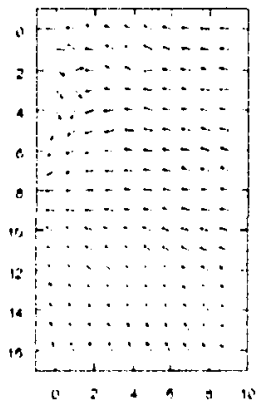

(b) original, horizon

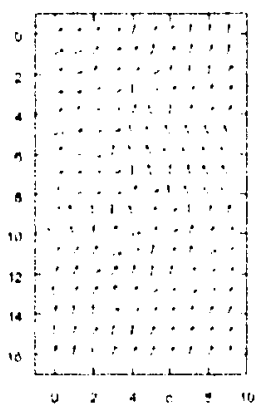

(e) hall1, horizon

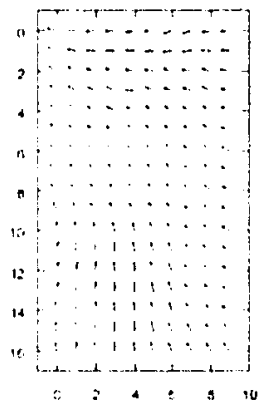

(h) hall2, horizon

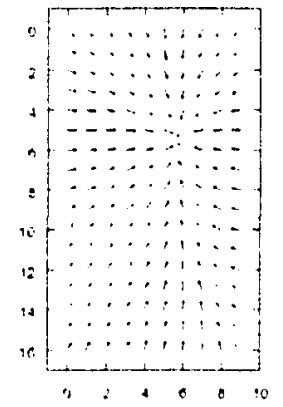

(c) original, above

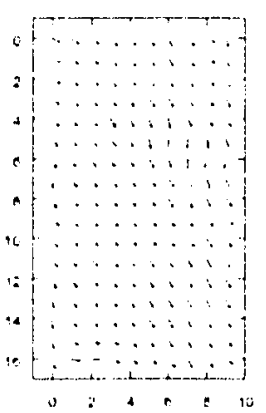

(f) hall1, above

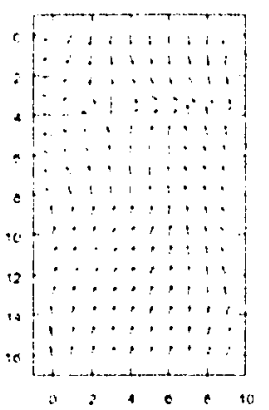

(i) hal12, above

Figure 4.10: Average landmark vector fields as computed by COMALV. Captions show the image collection and the location of the extracted region of the two-dimensional image. below: below the horizon, horizon $\pm 5^{\circ}$ around the horizon, above: above the horizon. 
Warping and COMALV. It shall be the policy throughout this thesis to refrain from making numerical comparisons between metrics outside the framework of a statistical test. Such tests will be presented for the methods described in this chapter in section 4.5 .

\subsection{Zeil et al., 2003: Gradient Descent}

In this section we review the findings of Zeil et al. who discovered that simple image differencing is useful for homing using panoramic snapshots of outdoor scenes [108]. They found that the difference between images varies smoothly and generally monotonically with distance. Thus, the snapshot location is a global minimum that can be reached by gradient descent. Zeil et al.'s method is remarkable for its simplicity and is included here for that reason. It is also notable for exhibiting good performance on outdoor images.

\subsubsection{Outdoor Scenes}

Zeil et al. reviewed a number of homing methods also reviewed here (including $[43,26,58$, $72,62]$ ) and point out that the most stringent test for any of these methods is to navigate within an indoor laboratory environment. They argue that such indoor environments lack the complexity of texture, contrast, occlusion, and depth of natural outdoor environments. This argument motivates their use of outdoor imagery: They employed a robotic gantry system to capture panoramic images from a sampling of outdoor environments. The images were generally captured within a cubic volume of about $1 \mathrm{~m}^{3}$.

\subsubsection{Image Differencing}

Zeil et al.'s purpose was to determine if simple image differencing could serve as a cue for homing in outdoor environments. The question they wished to answer was as follows:

"How different is the visual world when viewed from neighbouring vantage points, and is this difference correlated with, and docs it vary smoothly with. physical distance."

Their approach to this question was to employ the simplest possible means of judging the distance between images. The root mean square (RMS) difference value was calculated between a pair of images as follows. First, the pixel-by-pixel differences between the two images were squared. The root of the mean squared difference is then taken as the RMS value for that image pair. To test how RMS difference varies with physical distance, the 
RMS difference is calculated between images taken from different positions in space. With one such image designated as the reference or snapshot image, RMS differences are then determined at a number of positions surrounding the snapshot position. Plots of RMS difference show that it is indeed a smooth function of physical distance within most of the tested environments. These difference functions exhibited a prominent global minimum and a smooth, generally monotonic, increase in difference with distance. We will refer to a difference function with these properties as a smooth well. As will be demonstrated below, if a difference function is a smooth well then it can be used for homing by gradient descent.

Zeil et al. found difference functions to exhibit the smooth well property within a variety of conditions:

Height Difference functions were found to be smooth wells when the snapshot position and test positions were all within a plane of constant height above the ground. If the snapshot image was taken from a different height the smooth well property would still persist as long as the height difference was not too great.

Distance Most of the experiments in [108] used images taken within a $1 \mathrm{~m}^{3}$ cube. However, smooth wells were still found when the distance between snapshot and current image was increased by up to $3 \mathrm{~m}$.

Depth Smooth wells were found to persist within four different environments which varied in the depth of viewed objects. However, difference functions became less smooth in confined spaces with nearby large objects. In other experiments, it was found that close objects generally increase the steepness of the difference function.

Zeil et al. found not only that image differences increased smoothly with translation, but also with rotation. The RMS pixel differences between two images taken from different positions and orientations showed a clear minimum when both images had the same orientation. Also, the difference function increased smoothly with increasing angular distance between the two images. Thus, rotational difference functions were also found to be smooth wells. This result held for images taken within the same plane and when the snapshot image was taken from $1 \mathrm{~m}$ below the test positions. The maximum tested distance between images taken at different orientations was $1.22 \mathrm{~m}$.

If difference functions for both translation and rotation exhibit the smooth well property throughout an agent's working environment then simple algorithms for gradient descent can be used for visual homing, even if no compass is available. An agent displaced from home would first perform a one-dimensional gradient descent in rotation to align itself with the orientation it held when capturing the snapshot image. Then, the agent would translate in 
various directions and compute from these exploratory movements the direction of greatest descent. The agent would then make a movement in this direction.

Zeil et al. demonstrated gradient descent in translation using two simple algorithms. A variant of one of these will be used below to demonstrate gradient descent on the image database. First, however, we will examine the difference functions themselves in this image database and determine if the smooth well property holds in both translation and rotation.

\subsubsection{Gradients of Bielefeld Image Database}

\section{Translation}

Figure 4.11 shows the difference functions found between 20 representative snapshot images and all other images from collection original. As the images in the database were all taken from the same orientation, the differences between images are due only to translation.

The difference between two images is computed as the Sum of Squared Differences (SSD), not the Root Mean Squared (RMS) difference. In Zeil et al.'s experiments RNIS differences had to be used because the robotic gantry would appear in different parts of the image depending upon its position in space. This device was masked from the images prior to differencing. Thus, the images to be compared would vary in effective size, prompting the use of the mean squared difference. In our image database the images are all of the same size, therefore calculating the mean is umnecessary: Also, as we are only concerned with relative differences, calculating the root is unnecessary:

Figure 4.11 reveals that SSD also exhibits the smooth well property on these images for the majority of cases. This confirms the major finding of Zeil et al. in an indoor enviromment. These authors speculated that image differences might have been previously overlooked as a cue for navigation because of the local minima and abrupt changes induced by the corners and occluding edges of office enviromments. However, the SSD plots in figure 4.11 show a surprising smoothness in image differences, at least within one indoor office environment.

The bottom row, however, does reveal some exceptions. For the bottom right difference function, for example, there is a clear global minimum at the appropriate position $(9,16)$. However, there also appears to be a local minimum present in the upper right corner of the capture grid. Figure 4.12 shows again the difference functions for the bottom row of figure 4.11. However, in this plot the SSD values are raised to the power 4. Various local minima in these difference functions now become apparent. The presence of such local minima can impair gradient descent. 

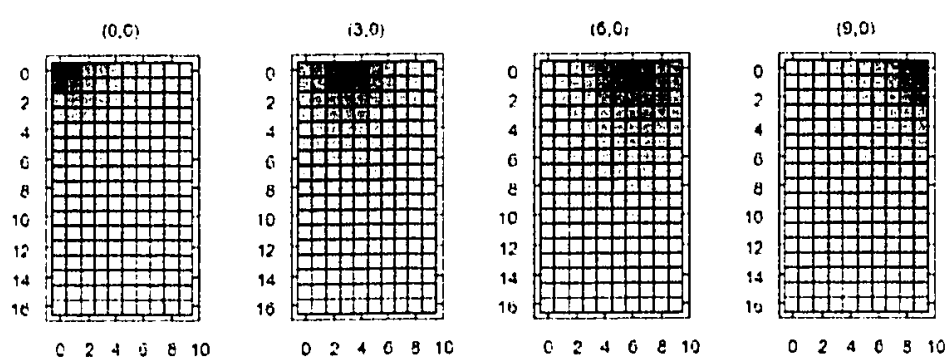

$(0.4)$

13,49

10.41
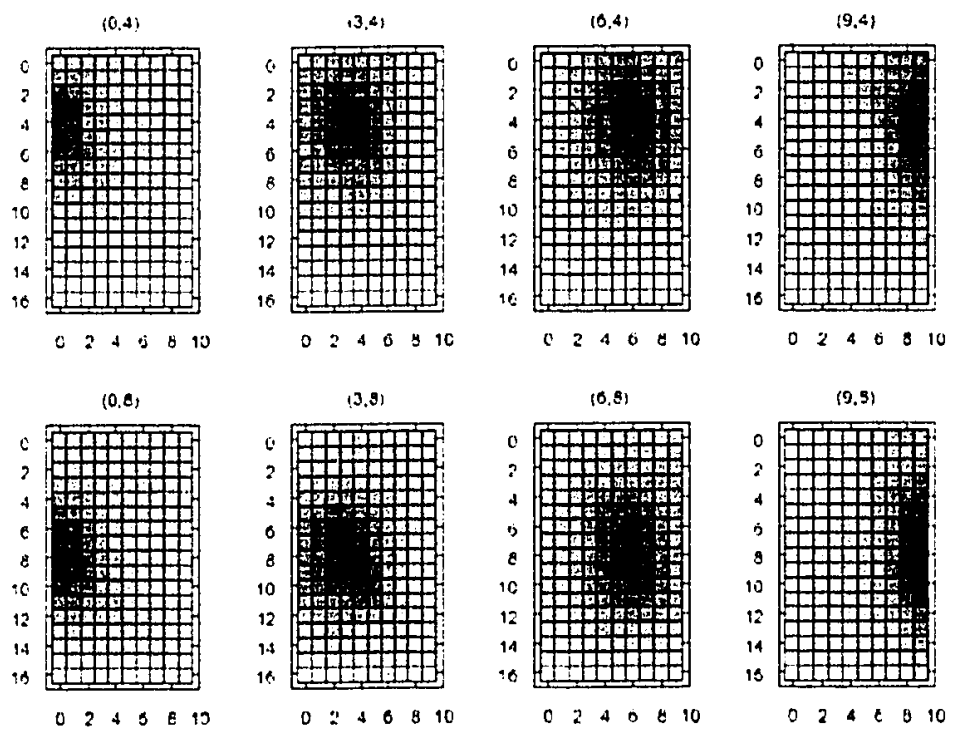

10.121

(3. 12,
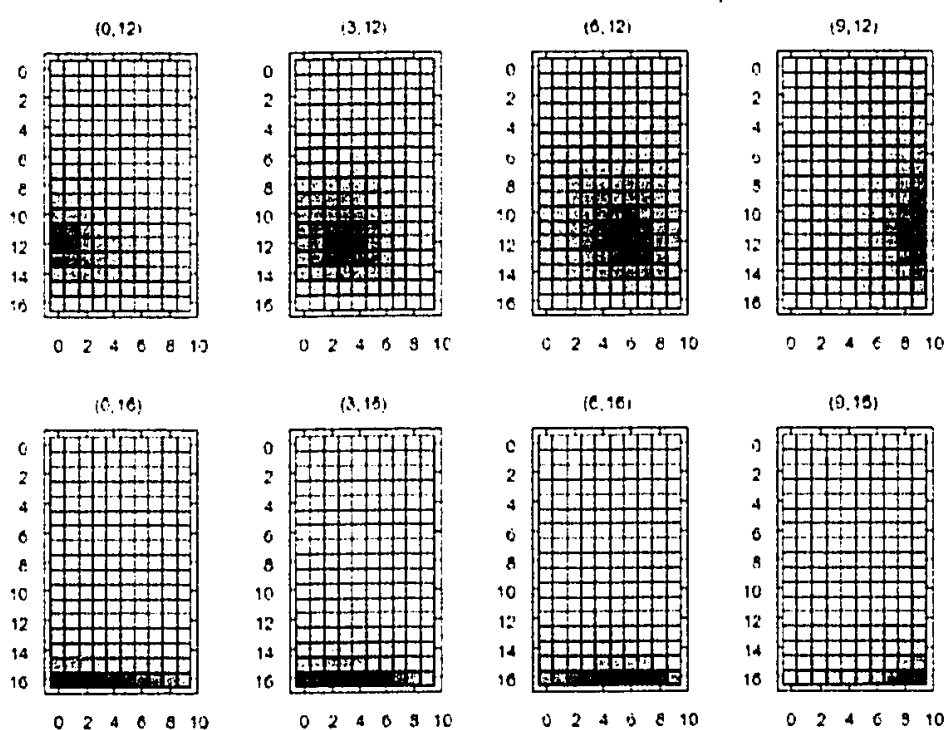

Figure 4.11: SSD between each of 20 snapshot images and all of the remaining 169 images of original. The position of the snapshot image used is indicated above each difference plot. Pure white indicates the highest SSD within each plot (the highest SSD here is 713.265). 

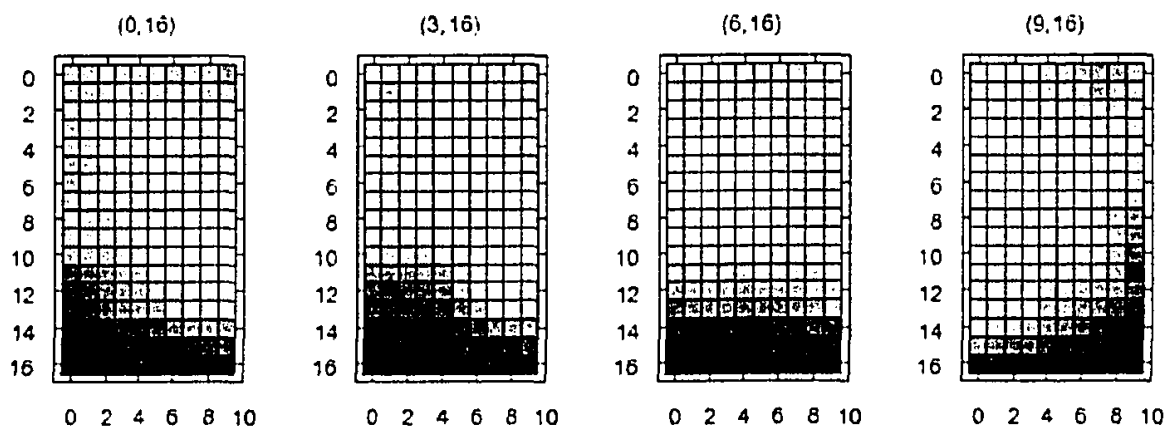

Figure 4.12: $\mathrm{SSD}^{4}$ between four snapshot positions along the bottom boundary of original and all other images from that collection.

\section{Rotation}

Figure 4.13 shows rotational difference functions between position $(3,4)$ of image collection original and four other positions. SSD was calculated while rotating the test images from $-180^{\circ}$ to $180^{\circ}$ in 44 increments. Also shown is the difference function between the snapshot image and rotated versions of itself. This trace dips down to zero as expected at $0^{\circ}$. The traces for the snapshot image and for positions $(0,0)$ and $(9,0)$ have a global minimum at zero. However, a very prominent local minimum exists for these three positions at about $180^{\circ}$. This bimodal distribution is clue to the fact that the office enviromment has two opposing walls which are white and relatively featureless. A $180^{\circ}$ reversal from the snapshot image pairs one white wall with its opposite counterpart, generating a local minimum. On the other hand, we can observe that these three traces are all smooth wells within the range $\left[-90^{\circ}, 90^{\circ}\right]$. However, this cannot be said for the remaining two traces. The traces for $(0,16)$ and $(9,16)$ do not have a minimum at $0^{\circ}$. These two locations are from the bottom portion of the capture grid. Figure 4.14(c) shows a manually selected division between the top and bottom portions of the capture grid. Figure 4.14(a) shows all difference functions for the top portion of the capture grid. The common global minimum at $0^{\circ}$ is evident. Figure 4.14(b) shows all difference functions for the bottom portion of the image database and the lack of a common minimum is similarly evident.

We can conclude that gradient descent in rotation is possible within some parts of the image database. However, the degree of rotation and the distance between current and snapshot location are limiting factors. 


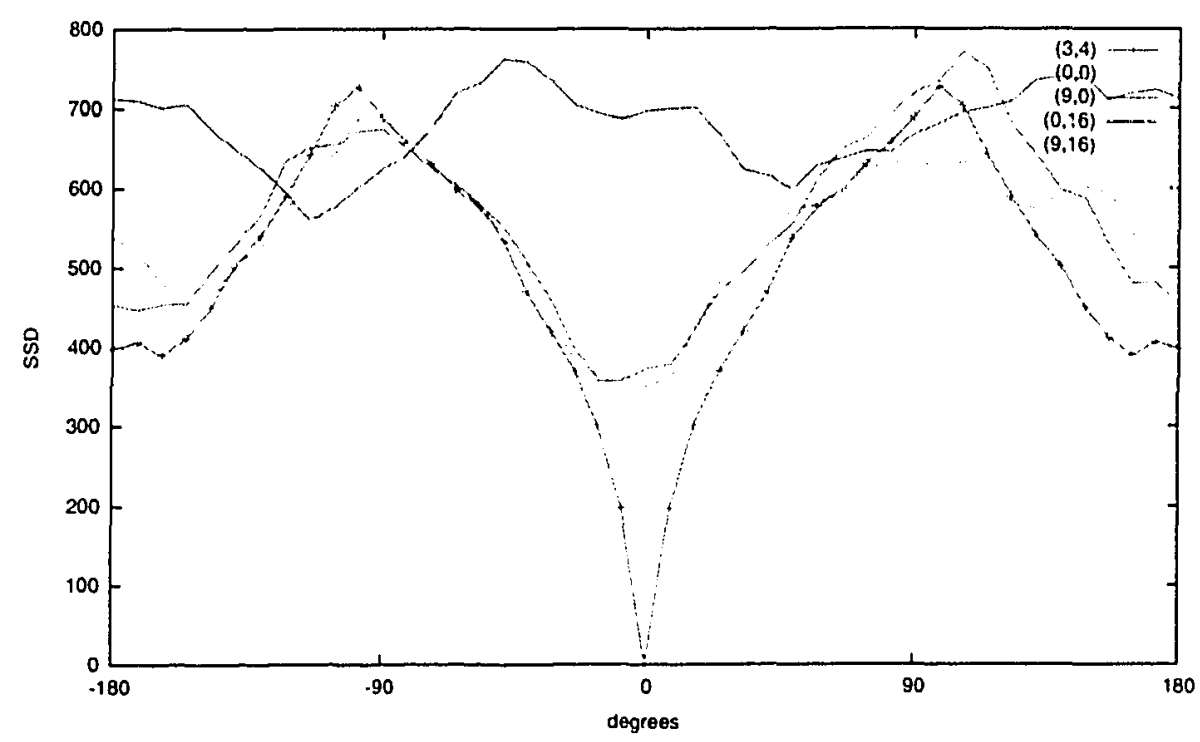

Figure 4.13: SSD between image $(3,4)$ of original and rotated versions of images $(3,4),(0,0),(9,0),(0,16)$, and $(9,16)$.

\subsubsection{Homing by Gradient Descent}

Zeil et al. tested two simple methods for homing by gradient descent on the difference functions between images. As in this thesis, they used images with constant orientation. These methods were tested online with the robotic gantry system controlled by the homing method. The first method, RunDown, begins by moving the camera in an arbitrary direction. Then, as soon as the RMS difference between the current image and snapshot image begins to increase, the movement direction is rotated counterclockwise by $90^{\circ}$. The camera then continues moving until RMS difference values again increase. RunDown, foregoes explorative movements which might allow the direction of gradient descent to be found more accurately. The second method, Triangular, makes two explorative moves such that it can compare the RMS values for three locations in space. It then moves in the direction of the minimum RMS value. Both methods were able to return the imaging device to within $5 \mathrm{~cm}$ of the snapshot position in 18 out of 20 runs. The time alloted for each attempt was 3 minutes. The 20 starting positions were positioned randomly within a $1 \mathrm{~m}^{2}$ area centred on the snapshot position. 


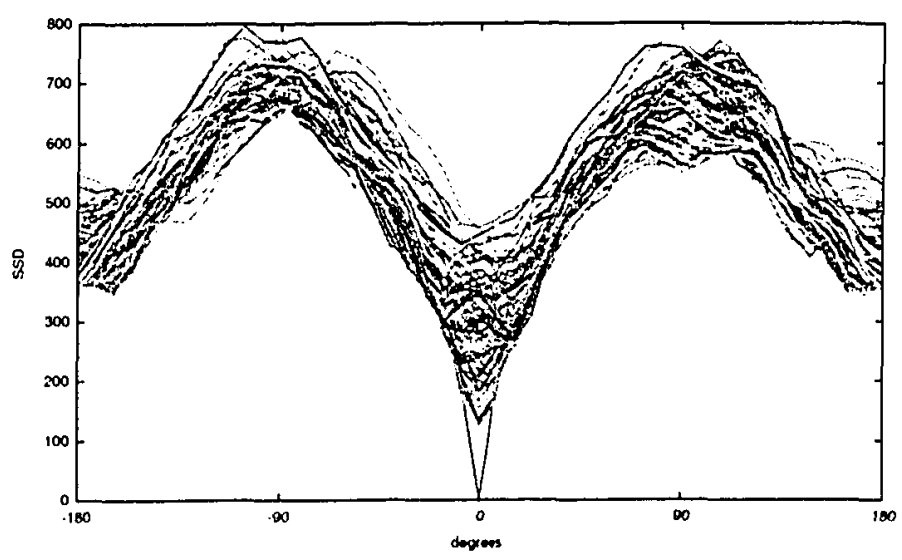

(a)

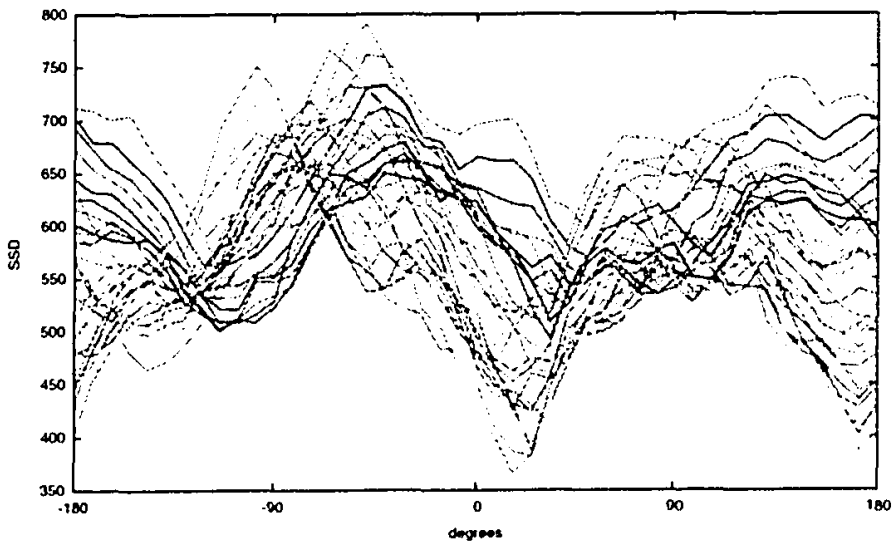

(b)

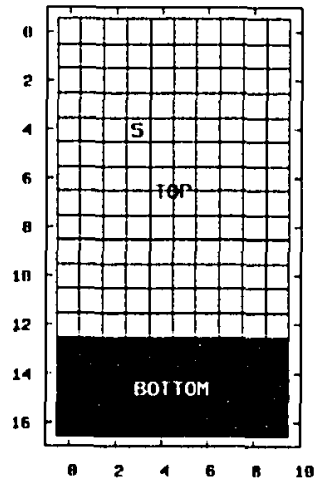

(c)

Figure 4.14: (a) SSD between image $(3,4)$ of original and rotated versions of all other images from the top portion of the image database. (b) As (a), however SSD is now calculated with the bottom portion of the image database. (c) Definition of the top and bottom portions of the image database. The ' $s$ ' label indicates the position of the snapshot image, $(3,4)$. 


\subsubsection{Tested Algorithm}

A direct implementation of either RunDown or Triangular is problematic on the image database because travel is not possible in all directions. Triangular, for example, requires two exploratory moves in arbitrary directions for each non-exploratory move. However difficulties persist even if the exploratory moves were to occur in line with the cardinal directions (left, right, up, and down). For instance, let us say that the method stipulates one exploratory move upwards, and one to the left. However, if the agent is on the upper or left boundary of the capture grid then one of these moves will not be possible. If the agent is in the upper left corner than neither move is possible.

Two variants of Triangular were developed for testing on the image database. Both variants make four exploratory moves before committing to a direction of movement. Each exploratory move involves a step in one of the four cardinal directions, and then a step back to the centre. In this manner, five SSD measurements can be taken-one measurement from the centre plus one from each exploratory move. For both variants the agent will stay still if the centre measurement is the smallest of the five. Ideally this situation should occur only at the goal position. The first variant, GradDescentMin will move in the direction of the smallest SSD measurement. Thus, it will only travel in one of the four cardinal directions. The second variant, GradDescent sums four weighted vectors, one for each exploratory move, where a vector's weight is given by the SSD in that direction. The sum of these vectors should point in the direction of greatest ascent. Therefore, the agent travels in the opposite direction, presumed to be the direction of greatest descent.

\subsubsection{Results on Image Database}

No results are provided for GradDescentMin as this method was found to be inferior in all cases to GradDescent.

\section{Image Collection original}

Figure 4.3 shows home vectors generated by GradDescent on image collection original for snapshot positions $(6,4)$ and $(0,16)$. With regards to snapshot position $(6,4)$ the home vector field has greater $A A E$ than either Warping or COMALV. However, like both of these methods the $R R$ for GradDescent is at the maximum value of one. The higher angular error reflects how this home vector field differs from the ideal field, but the return ratio reflects that this field may still be perfectly sufficient for homing. For snapshot position $(0,16)$ the home vector field for GradDescent is less impressive. While many home vectors in the 
region just above s are approximately correct, those along the bottom remain incorrect. If a homing agent were able to perceive that it had reached the end of the capture grid it could then constrain itself to travel only along the boundary. In this manner, $\mathbf{s}$ might be reached via the vector field depicted in figure 4.3(f). However, such a method is not applicable here as the boundary is only the spatial limit of the capture grid and does not correspond with any possible percept.

Figure 4.4 plots $A A E$ and $R R$ across snapshot positions. Both plots show that the bottom boundary is a problem area for GradDescent. As revealed in figure 4.12 additional local minima exist for difference functions in this area. These local minima impair homing to snapshot positions along the line $y=16$.

\section{Image Collections hall1 and hall2}

Figure 4.5 shows the home vectors generated by GradDescent in hall1 and hall2 for snapshot position $(3,12)$. These home vector fields, exhibit regions around the snapshot position where the home vectors are qualitatively correct. However, the quality of home vectors in the top portion of the capture grid is generally poor.

Figures 4.7 and 4.8 show relatively consistent performance of GradDescent across snapshot positions in hall1 and hal12. Performance at central positions such as $(3,8)$ and $(6,8)$ slightly exceeds that found in neighbouring and boundary positions. This finding is consistent with Zeil et al.'s finding that nearby objects can impact the smoothness of difference functions.

\section{Cross-Collection Test}

The results of the cross-collection test, shown in figure 4.9, indicate that GradDescent is sensitive to some envirommental modifications more than others. Like Warping and COMALV, GradDescent performs quite poorly when either doorlit or winlit is used for snapshot images and any other collection is used for current images. However, GradDescent also performs relatively poorly when current images are taken from chairs or day.

\subsection{Comparative Results}

The results presented so far describe the three methods on an individual basis. The only comparisons made between methods so far have been qualitative in nature. In this section we present the results of statistical tests which compare the average $A A E$ and $R R$ between methods. As described in section 3.3.5, the tests used are the repeated measures ANOVA 
with Tuley-Kramer post-tests for $A A E$, and Friedman's test with Dumn's post-tests for $R R$.

Table 4.1 presents the results of these tests on image collection original. According to the top half of the table, COMALV exhibits a significantly lower $A . A E$ than the other two methods. The difference in $A A E$ between Warping and GradDescent is not significant. However, the bottom half of the table indicates that no significant differences were found among the $R R$ values of the three methods.

Table 4.2 presents the results for hall1 and tells a very different story. Here both Warping and GradDescent outperform COMALV in both performance metrics. Also, Warping exhibits a significantly lower $A A E$ than GradDescent, however the difference in $R R$ between these two methods is not significant. The results are nearly the same in hall2 (see table 4.3), with the only change being that the difference in $A A E$ between COMALV and GradDescent is no longer significant.

The table for the cross-collection test, table 4.4 shows less agreement between $A A E$ and $R R$. For both metrics, Warping does better than GradDescent. However, in angular error COMALV does better than both Warping and GradDescent, while in return ratio both Warping and GradDescent perform better than COMALV.

As a guide in interpreting tables such as these, the reader is suggested to gange the strength of each method by the number of marked cells in the row for that method. For example, in the top portion of table 4.1 the row for COMALV has the maximum of two cells marked, indicating that it is the strongest method on image collection original. Similarly, the number of marked cells in a column shows the wcakness of that column's method. For example, in table 4.2 the column for COMALV has the maximum of two cells marked, indicating that it is the weakest method on image collection hal11. Note that this interpretation of these tables is suggested here only to help orient the reacler. The number of marked cells in a row or column does not provide a statistically valid means to rank the methods.

What may be concluded from these differing results? We can observe that GradDescent is never in a position to outperform the other two methods for either metric in any of the four tests. Using the AAE metric, COMALV is the best method in both original and in the cross-collection test. However, using the $R R$ metric, all methods are equivalent in original and Warping is the best in the cross-collection test. Moreover, Warping is unambiguously the best method in both hall1 and hal12, while COMALV is either the worst or tied for the worst. Overall, it must be concluded that Warping exhibits the most robust performance of the three methods considered in this chapter. 
Significance of difference in $A A E_{*}$ (original)

\begin{tabular}{|l|l|l|l|}
\hline & Warping & COMALV & GradDescent \\
\hline Warping & $\mathrm{X}$ & & \\
\hline COMALV & $* *$ & $\mathrm{X}$ & $* *$ \\
\hline GradDescent & & & $\mathrm{X}$ \\
\hline
\end{tabular}

Significance of difference in $R R_{*}$ (original)

\begin{tabular}{|l|l|l|l|}
\hline & Warping & COMALV & GradDescent \\
\hline Warping & $\mathrm{X}$ & & \\
\hline COMALV & & $\mathrm{X}$ & \\
\hline GradDescent & & & $\mathrm{X}$ \\
\hline
\end{tabular}

Table 4.1: Statistical significance of the difference in $A A E_{*}$ and $R R_{*}$ between homing: methods. Significance for each cell is indicated if the method on the vertical axis is significantly better (lower $A A E$ or higher $R R$ ) than the method on the horizontal axis. Empty fields indicate no significant difference. For $A A E$ a repeated measures ANOVA with Tukey-liramer post-test was used. For $R R$ Fliedman's test with Dunn's post-test was used. Legend: ${ }^{*}=(p<0.05),{ }^{* *}=(p<0.01),{ }^{* * *}=(p<0.001),{ }^{* * * *}$ $=(p<0.0001), \mathrm{X}=$ self-match.

Significance of difference in $A A E_{*}($ hall1)

\begin{tabular}{|l|l|l|l|}
\hline & Warping & COMALV & GradDescent \\
\hline Warping & $\mathrm{X}$ & $* * *$ & $* * *$ \\
\hline COMALV & & $\mathrm{X}$ & \\
\hline GradDescent & & $* *$ & $\mathrm{X}$ \\
\hline
\end{tabular}

Significance of difference in $R R_{*}$ (hall1)

\begin{tabular}{|l|l|l|l|}
\hline & Warping & COMALV & GradDescent \\
\hline Warping & $\mathrm{X}$ & $* * *$ & \\
\hline COMALV & & $\mathrm{X}$ & \\
\hline GradDescent & & $* *$ & $\mathrm{X}$ \\
\hline
\end{tabular}

Table 4.2: Statistical significance of the difference in $A A E_{*}\left(\right.$ hall1) and $R R_{*}$ (hall1) between homing methods. See table 4.1 for notation. 
Significance of difference in $A A E_{*}(\mathrm{hall2})$

\begin{tabular}{|l|l|l|l|}
\hline & Warping & COMALV & GradDescent \\
\hline Warping & $\mathrm{X}$ & $* * *$ & $* * *$ \\
\hline COMALV & & $\mathrm{X}$ & \\
\hline GradDescent & & & $\mathrm{X}$ \\
\hline
\end{tabular}

Significance of difference in $R R_{*}$ (hall2)

\begin{tabular}{|l|l|l|l|}
\hline & Warping & COMALV & GradDescent \\
\hline Warping & $\mathrm{X}$ & $* * *$ & \\
\hline COMALV & & $\mathrm{X}$ & \\
\hline GradDescent & & $* *$ & $\mathrm{X}$ \\
\hline
\end{tabular}

Table 4.3: Statistical significance of the difference in $A E_{*}$ (hal12) and $R R_{*}$ (hal12) between homing methods. See table 4.1 for notation.

Significance of difference in $A A E_{*}(*, *)$

\begin{tabular}{|l|l|l|l|}
\hline & Warping & COMALV & GradDescent \\
\hline Warping & $\mathrm{X}$ & & $* * *$ \\
\hline COMALV & $* * *$ & $\mathrm{X}$ & $* * *$ \\
\hline GradDescent & & & $\mathrm{X}$ \\
\hline
\end{tabular}

Significance of difference in $R R_{*}(*, *)$

\begin{tabular}{|l|l|l|l|}
\hline & Warping & COMALV & GradDescent \\
\hline Warping & $\mathrm{X}$ & $* * *$ & $* * *$ \\
\hline COMALV & & $\mathrm{X}$ & \\
\hline GradDescent & & $*$ & $\mathrm{X}$ \\
\hline
\end{tabular}

Table 4.4: Statistical significance of the difference in $A E_{*}(*, *)$ and $R R_{*}(*, *)$ between homing methods for all pairings of inage collections from the office environment. See table 4.1 for notation. 


\subsection{Complexity and Biological Plausibility}

\subsubsection{Warping}

Warping involves a search process and therefore does not satisfy the constraints of retinotopic processing introduced in chapter 1. However, the fact that Warping operates on one-dimensional images makes it tractable for real-time operation. The averaged processing time per image on a Pentium $42 \mathrm{GHz} \mathrm{PC}$ was $16.8 \mathrm{~ms}^{6}$.

The number of pixel-to-pixel comparisons required for Warping is:

$$
\mathcal{C}_{\text {Warping }}=n_{n} n_{\rho} n_{\vartheta^{*}}\left\lfloor w / m_{x}\right\rfloor
$$

For the parameters selected in section 4.2 .6 this quantity comes to $64^{2} \times 206 \approx 850,000$ operations. Franz et al. argue that their method could conceivably be implemented in parallel through a set of matched filters, akin to those described for optic flow interpretation in insects $[55,54,22]$. However, 850,000 operations would still be required, and if they were all to occur in parallel using at least one neuron per operation then Warping appears rather infeasible for implementation in the brain of an insect. As mentioned in chapter 1, insects such as bees and ants have on the order of one million neurons, and they have many more uses for these neurons then just visual homing. Note that Möller [74] came to a similar conclusion using a different set of parameters for Warping.

\subsubsection{COMALV}

COMALV is an extraordinarily simple and low-cost homing method. The method's arerage processing time on the computer mentioned above was only about one millisecond. The method's first step is the mapping between two-dimensional images and one-dimensional images, which is a convergent summing operation. Next, Gaussian filtering and downsanpling are applied, both of which are retinotopic-compatible. Then, at both the snapshot and current positions equation (4.8) must be computed to obtain the average landmark rector for that position. This equation can be applied in parallel at every pixel in the snapshot image and is therefore also retinotopic-compatible. Finally, the vector difference of equation (4.9) is computed as a global operation to obtain the final home vector. As this one global operation is so simple, COMALV easily satisfies the retinotopic processing constraints.

\footnotetext{
${ }^{6}$ The same computer was used for all processing times reported in this thesis. Estimates of average processing time were obtained by processing all images from image collection original and dividing by the number of images in this collection $(170)$.
} 


\subsubsection{GradDescent}

The computational effort required by GradDescent is also very low. The processing time is similar to COMALV. First, for every image the SSD must be computed. This involves finding per-pixel differences and squaring them, which are retinotopic-compatible operations. A convergent summing operation then yields the SSD for that image. There are also costs related to determining the home vector from the SSD values at the centre and the four explored positions. For each explored position, a vector is directed from the centre towards the direction of exploration. Arriving at the direction of exploration requires that the path integration system be consulted. Finally, these four vectors are weighted by the relevant SSD values and summed. It is reasonable to consider this vector sum as a simple global operation. Thus, GradDescent satisfies the retinotopic processing constraints.

However, GradDescent also incurs other costs beyond computational ones. For every non-exploratory move of the agent, four exploratory moves must be spent. Assuming that the distance moved for an exploratory move is the same as for a non-exploratory move, GradDescent will need to move eight times as far as another method which follows the same course to the goal. Of course, this would entail an eightfold increase in time and energy spent as well. However, the balance between distance moved in exploration versus distance moved in travel towards the goal need not be so lopsided. On the image database, the distance travelled for an exploratory move must be at least one. However, with a free-roving robot, the distance travelled for an exploratory move could be set to any value. On such a platform, determining the minimum useful exploratory move length would be an empirical cquestion. Also, on a robotic platform the choice of the number and direction of exploratory moves would be open to investigation. Indeed, Zeil et al. tested two schemes which both required fewer exploratory moves than GradDescent. In order to approximate the local gradient of the difference function the SSD for at least three positions is required, where the line between the first and second positions should be orthogonal to that between the second and third. Making one exploratory move orthogonal to the previous non-exploratory move would reduce the ratio of exploratory to non-exploratory moves to 1:1. Nevertheless, the accuracy at which the local gradient is estimated will depend upon the distance, direction, and number of exploratory moves. Thus, there exists a tradeoff between accuracy and exploratory movement. 


\subsection{Conclusions}

This chapter has thoroughly reviewed three modern visual homing methods which have all been shown to perform well on real-world images while having some claim to biological plausibility. As the performance criterion is primary, it is concluded that Warping is the method that should be selected for comparison with subsequent homing methods. COMALV exhibited impressive performance on the original image collection, but its poor performance on the hall collections suggest that the centre-of-mass property may not exist for all environments, or that a centre-of-mass detector would require customization for particular environments. GradDescent was found to perform robustly; but always less successfully than at least one of the other methods. GradDescent also requires exploratory moves, although the cost of these moves may well be significantly reduced. 


\section{Chapter 5}

\section{Vector Mapping}

\subsection{Introduction}

Feature-based approaches to visual homing require some way of computing a home vector from the correspondence vectors which relate image features in the snapshot image, $S$, to those in the current image, $C$. Correspondence vectors have their tails at features in the snapshot image, $S$, and their heads at the corresponding features in the current image, $C$. These vectors are expressed in the image plane. However, home vectors are expressed in the plane of travel. We define vector mapping as the process of finding home vectors from correspondence vectors.

In this chapter we review a previous one-dimensional technique for vector mapping which relies upon making a particular assumption about the distribution of landmarks in the enviromment. We go on to develop a new two-dimensional technique which requires no such assumption. This method has been described in [98].

\subsection{Notation}

In this chapter it is most convenient to use angles in a spherical coordinate system as image indices, as opposed to integer indices. At the snapshot position $s$, a landmark 1 in the environment is imaged onto the unit sphere at position $\theta=\left(\theta_{x}, \theta_{y}\right)$. Thus, $\theta$ is a position within image $S$. Note that the actual images used have a limited resolution. Therefore, the coordinates $\left(\theta_{x}, \theta_{y}\right)$ will actually be integer multiples of $\Delta_{x}$ and $\Delta_{y}$ where $\Delta_{x}$ represents the inter-pixel angle in the horizontal direction, and $\Delta_{y}$ represents vertical inter-pixel angle.

At the current position c some correspondence method (unspecified in this chapter) is 
employed to find the same landmark 1 in $C$. Let this corresponding position be $\check{\theta}=\left(\check{\theta_{x}}, \check{\theta_{y}}\right)$. We now define the correspondence vector $\delta$,

$$
\delta=\left(\begin{array}{l}
\delta_{x} \\
\delta_{y}
\end{array}\right)=\left(\begin{array}{l}
\check{\theta}_{x}-\theta_{x} \\
\check{\theta}_{y}-\theta_{y}
\end{array}\right)
$$

\subsection{Hong et. al's 1-D Technique}

All feature-based approaches to visual homing must have some means to map correspondence vectors to home vectors. The majority of visual homing methods reviewed in chapter 1 utilize one-dimensional images, and therefore use only the $x$ component of the correspondence vector, $\delta_{x}$ (an interesting exception is the original snapshot model, which uses vectors to correct for the bearing of image regions, as well as vectors to correct for size changes between regions). One-dimensional techniques are conceptually simple and easy to illustrate, however the home vectors they produce are necessarily only approximations to the true home vector due to the lack of available depth information.

Franz et al. review a number of visual homing methods and find the vector mapping technique of Hong et al. to be employed in several other methods [26, 43]. We use this technique here as a representative example of one-dimensional vector mapping.
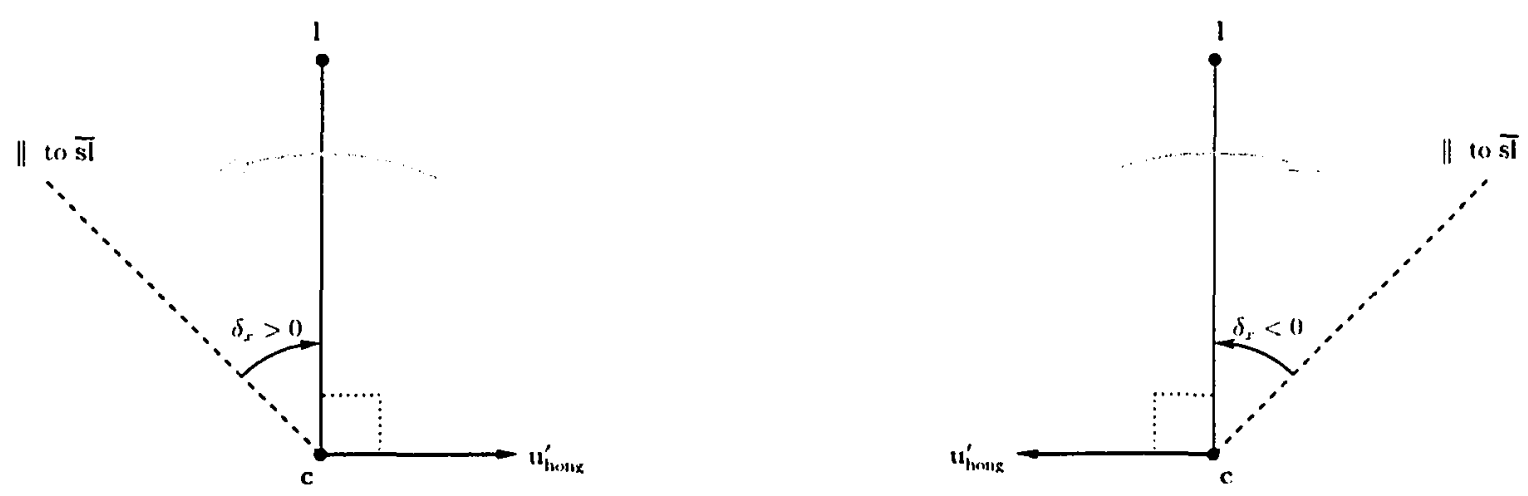

Figure 5.1: Illustration of the main idea behind Hong et al.'s vector mapping technique. On the left, $\delta_{x}$ is positive, meaning that the current view of landmark $l$ is clockwise of its view at the snapshot position. The gray curved arrow indicates how the view of 1 would change if the agent moved in direction $\mathbf{u}_{\text {hong }}^{\prime}$. On the right the situation for negative $\delta_{x}$ is presented.

Assume that we are at the current position $\mathrm{c}$ and we observe landmark 1 . At the goal 
position, landmark 1 was visible along the line $\overline{\text { sl. }}$. The idea of Hong et al.'s vector mapping technique is to move so that the current view of landmark 1 shifts towards the view held at the goal. Figure 5.1 shows two different scenarios. On the left of the figure $\delta_{x}$ is positive, meaning that the current view of landmark I should shift counterclockwise. On the right $\delta_{x}$ is negative, meaning that a clockwise shift is required. Hong et al. observed that these shifts can be achieved by moving in a direction perpendicular to $\mathrm{l}$ as follows:

$$
\begin{array}{ll}
\theta_{x}+\pi / 2 & \text { if } \delta_{x}>0 \\
\theta_{x}-\pi / 2 & \text { if } \delta_{x}<0
\end{array}
$$

Let $u_{\text {hong }}^{\prime}$ be a vector directed according to the above conditions. Movement in the direction of $\mathbf{u}_{\text {hong }}^{\prime}$ creates the appropriate shifts, visualized as the gray curved arrows in figure 5.1.

Hong et al.'s technique incorporates several refinements on the basic idea described above. The first, rather subtle, refinement comes from the observation that whatever method we use to compute a home vector from $\mathbf{c}$ to $\mathbf{s}$, we can reverse the roles of $\mathbf{c}$ and $\mathbf{s}$ (as well as $C$ and $S$ ) and compute an alternate vector which should ideally be opposite to the true home vector. Hong et al. compute this alternate vector and average its opposite with the 'normal' home vector. Incorporating this refinement, the home vector $\mathbf{u}_{\text {hong }}$ has a direction as follows:

$$
\angle \mathbf{u}_{\text {hong }}= \begin{cases}\theta_{x}+\pi / 2+\delta_{x} / 2 & \text { if } \delta_{x}>0 \\ \theta_{x}-\pi / 2+\delta_{x} / 2 & \text { if } \delta_{x}<0\end{cases}
$$

Figure 5.2 illustrates this direction. As the figure shows, $u_{\text {hong }}$ is clirected in the approximate direction of home. The error angle $\epsilon$ shows the deviation from the true home direction. Note that we let $\mathbf{u}_{\text {hong }}$ be a unit vector.

To compute the final home vector, all home vector estimates $\mathbf{u}_{\text {hong }}$ are first summed together.

$$
\mathbf{v}_{\text {liong }}=\sum_{i}^{n} \mathbf{u}_{\text {long }}^{i}
$$

where the summation is over all $n$ correspondence vectors. Finally; $\mathbf{v}_{\text {hong }}$ is normalized

$$
\hat{\mathbf{v}}_{\text {hong }}=\frac{\mathbf{v}_{\text {hong }}}{\left\|\mathbf{v}_{\text {hong }}\right\|}
$$

The vector $\hat{\mathbf{v}}_{\text {hong }}$ above is the final home vector.

An additional refinement on this technique involves scaling the individual home vectors $\mathbf{u}_{\text {hong }}$ by $\delta_{x}$. As explained by Hong et al., errors in $\delta_{x}$ are likely to cause oscillation between the two conditions in equation (5.3) when $\delta_{x}$ is small. When $\delta_{x}$ is large the computed home vectors are less sensitive to error. Thus, scaling by $\delta_{x}$ allows more weight to be placed on home vectors with larger $\delta_{x}$ in the summation of equation (5.4). In fact, additional terms are added to the scale factor [43]. However, following Franz et al, we address only $\delta_{x}$. 


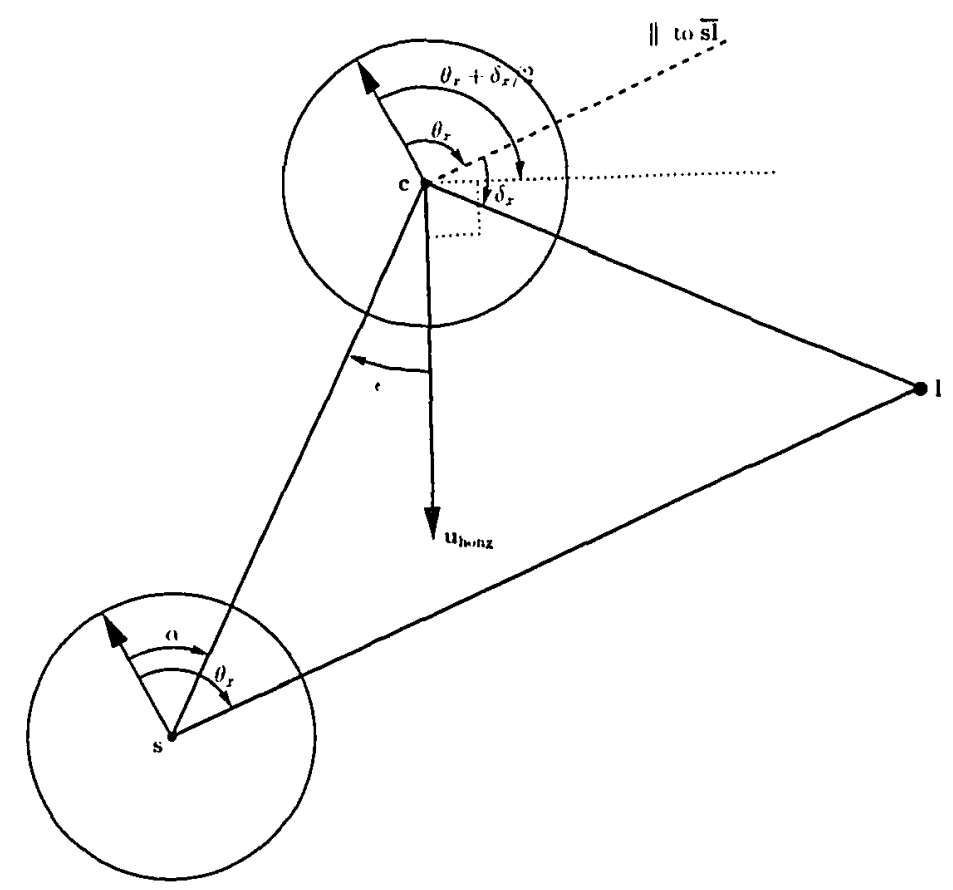

Figure 5.2: Illustration of the direction of home vector $\mathbf{u}_{\text {hong }}$ as computed by Hong et al.'s vector mapping technique.

\subsubsection{Correctness}

The argument is made by Franz et al. that Hong et al.'s approach implicitly relies upon an isotropic distance assumption, meaning that "the frequency' and clistance of landmarks are assumed to be independent of the viewing direction" [26]. For each landmark a home vector estimate, $\mathbf{u}_{\text {hong }}$, is computed. This home vector will generally contain an error component orthogonal to the true home direction. Franz et al. argue that if the isotropic distance assumption is true then all error components will cancel out in the summation of equation $(5.4)^{1}$.

Further, Franz et al. show that Hong et al.'s vector mapping technique is never wrong by more than $\frac{\pi}{2}$, provided that $\delta_{x}$ is error-free. They go on to prove that as long as the angular error is bounded by $\frac{\pi}{2}$, the homing agent must eventually converge to the goal.

\footnotetext{
${ }^{1}$ It would seem, however, that an even stronger assumption would be required for this to hold true. This stronger assumption would stipulate that for every landmark that generates a home vector with an error component, there will be another landmark at just the right position to generate a home vector with an opposite error component. In this way all error components would cancel each other out exactly.
} 
In essence, the distance to the goal $b$ must decrease monotonically if $|\epsilon|<\frac{\pi}{2}$, therefore $\lim _{t \rightarrow \infty} b(t)=0$, where $t$ is time. Thus, $\frac{\pi}{2}$ has a special consequence for homing. If the angular error is always less than $\frac{\pi}{2}$ then homing will be convergent. Having an average angular error less than $\frac{\pi}{2}$ does not indicate convergent homing. Nevertheless, we will make some reference to $\frac{\pi}{2}$ as a threshold between good and bad homing performance.

\subsection{Vardy \& Möller's 2-D Technique}

Previous approaches to vector mapping such as Hong et al.'s operate exclusively on onedimensional images. However, there is a great deal of information lost in the mapping from two-dimensional images to one-dimensional images. Consider the view of a lamp from the perspective of a person walking about in a room. The lamp's position on the retina certainly shifts horizontally as one moves, but it also shifts vertically: The objects of interest for Hong et al.'s approach are those lying within the plane of travel. For this example, only a lamp at eye level would be of interest. However, this is a degenerate case. It would be preferable to glean useful information from all visible objects, not just those which happen to intersect the plane of travel. Incleed, it turns out that by considering the motion of image features in two dimensions a one-to-one mapping between correspondence vectors and home vectors can be established.

Figure 5.3(a) shows a top-down view of an agent at its current position c. the goal position $\mathrm{s}$, and a landmark 1 visible from both positions.

We assume that the agent has some form of compass to allow it to restore the orientation held at $\mathbf{s}$. The translation from $\mathrm{s}$ to $\mathrm{c}$ occurred at an angle $a$ from the agent's heading (indicated by the arrow). At $s$, the angular position of $l$ in the agent's field of view was $\theta_{x}$. At $\mathrm{c}$, the view of 1 has shifted by $\delta_{x}$. The distances $d_{\mathrm{s}}$ from $\mathrm{s}$ to $\mathrm{l}$, and $d_{\mathrm{c}}$ from $\mathrm{c}$ to 1 are included in the figure, however both are unknown.

The correct direction home is given by $\beta=a+\pi$. We wish to compute 3 given the correspondence vector $\delta$ and the projection of 1 in the snapshot image, given by $\left(\theta_{x}, \theta_{y}\right)$. Unfortunately, with the situation described thus far, the lengths of all three sides of triangle $\triangle \mathrm{lsc}$ remain unknown. If we have no information about these lengths then it becomes impossible to compute $\beta$ exactly. As a means of circumventing this dilemma Franz et al. proposed two assumptions about the distribution of landmarks in the environment which allow $\beta$ to be approximated [26]. However, no such assumptions are required if we can just determine the ratio between the distances $d_{\mathbf{s}}$ and $d_{\mathbf{c}}$. This ratio can be obtained by considering the vertical components $\delta_{y}$ and $\theta_{y}$. 


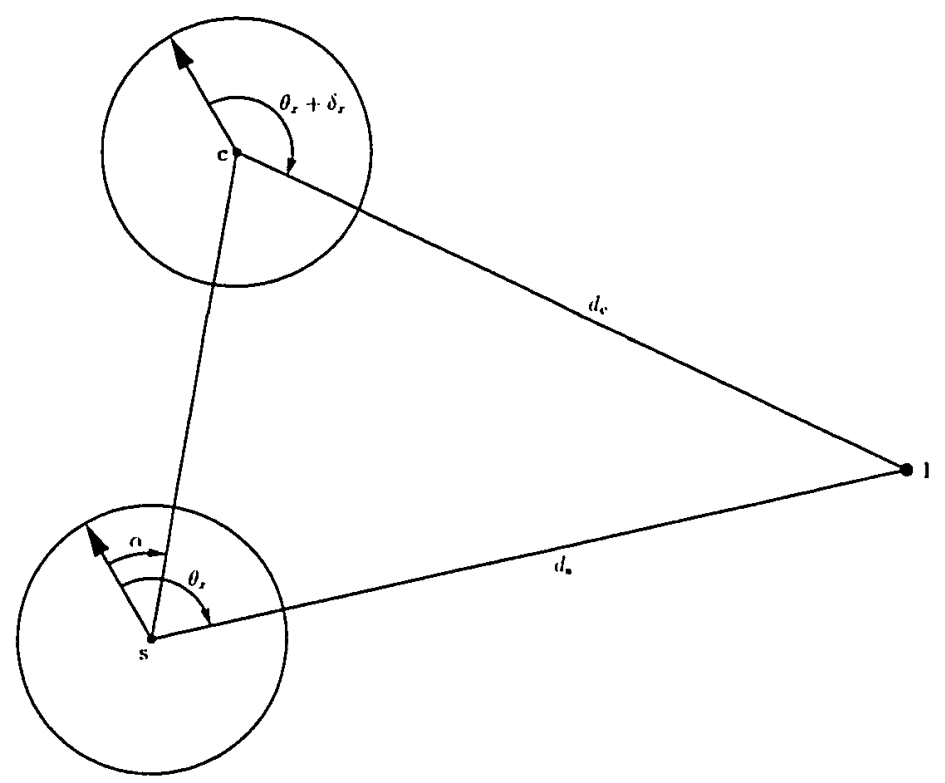

(a)

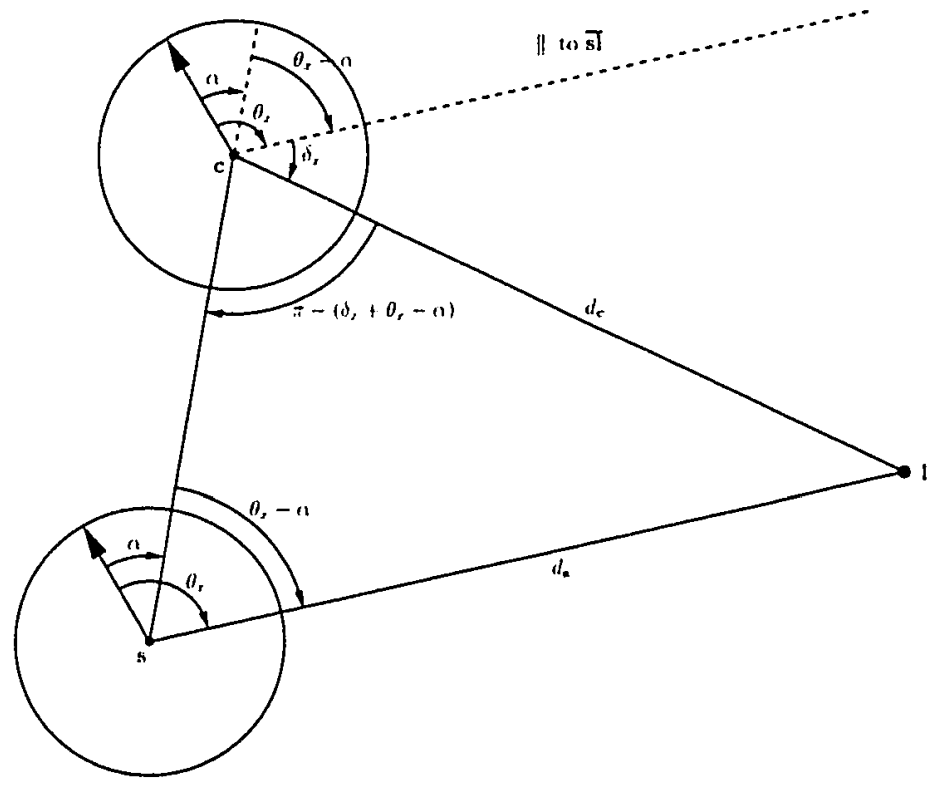

(b)

Figure 5.3: Motion of an agent from $\mathrm{s}$ to $\mathrm{c}$ and the resultant change in viewing position of a landmark 1 . 


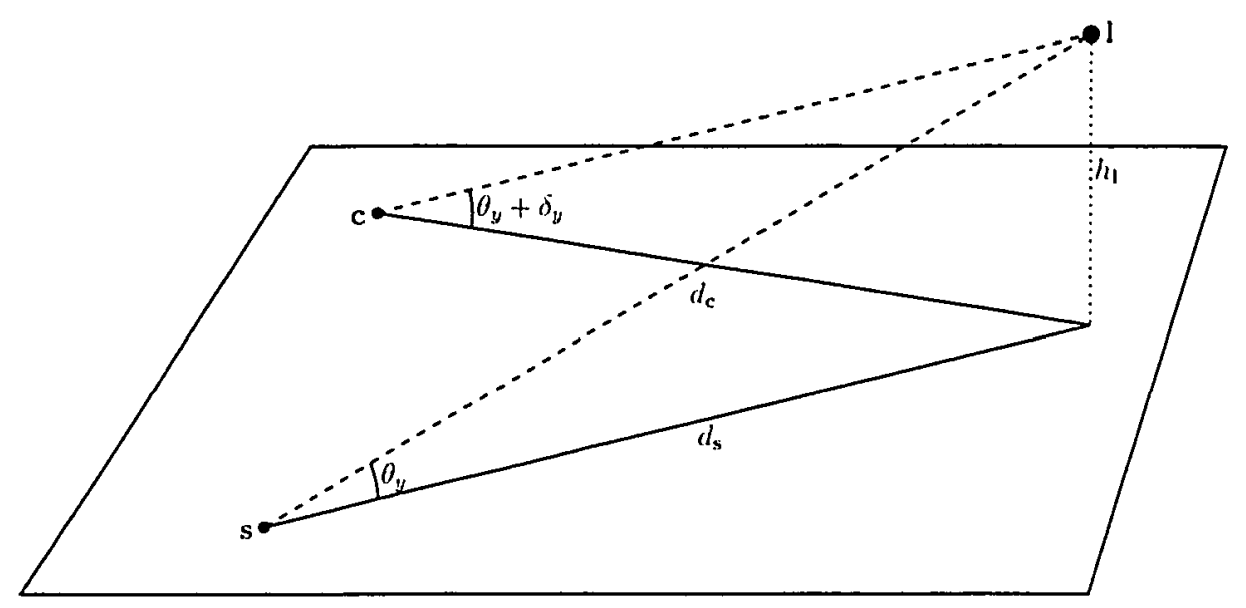

Figure 5.4: Oblique view of plane containing $\mathrm{s}$ and $\mathrm{c}$. Solid lines are within the plane, dashed lines are above and oblique to the plane, and the dotted line is perpendicular to the plane.

Figure 5.4 shows $\mathbf{s}, \mathbf{c}$, and $\mathbf{l}$ when viewed from an oblique angle above the plane containing $\mathbf{s}$ and $\mathbf{c}$. The agent is constrained to travel such that the centre of its imaging sphere lies within the plane (and thus the plane projects on the image horizon). Landmark 1 lies a perpendicular distance $h_{1}$ above (or below) the plane. This picture and the use of the vertical components $\delta_{y}$ and $\theta_{y}$ allow us to calculate the ratio $d_{\mathbf{s}} / d_{\mathbf{c}}$. First we find the tangents for the two angles of 1 above the plane.

$$
\begin{aligned}
\tan \theta_{y} & =\frac{h_{1}}{d_{\mathbf{s}}} \\
\tan \left(\theta_{y}+\delta_{y}\right) & =\frac{h_{1}}{d_{\mathbf{c}}}
\end{aligned}
$$

We now eliminate $h_{1}$ to obtain the desired ratio

$$
\frac{d_{\mathbf{s}}}{d_{\mathbf{c}}}=\frac{\tan \left(\theta_{y}+\delta_{y}\right)}{\tan \theta_{y}}
$$

Note that equation (5.8) becomes numerically unstable when $\theta_{y}$ is close to zero. This makes intuitive sense. We cannot reliably compute the ratio $d_{\mathrm{s}} / d_{\mathrm{c}}$ if the landmark 1 lies on the horizon. In the implementation of this vector mapping technique points very near the horizon are omitted $\left(\left|\theta_{y}\right| \leq 0.01\right)$.

Knowing how to compute the ratio $d_{\mathbf{s}} / d_{\mathbf{c}}$ we return to the analysis of horizontal angles with the goal of deriving an expression for $\beta$. Figure 5.3(b) is identical to figure 5.3(a) 
except that it shows a number of intermediary angles that will be useful below. We begin by determining the interior angles of the triangle at $\mathrm{s}$ and $\mathrm{c}$. $\angle \mathrm{s}$ is just $\theta_{x}-\alpha$. To determine $\angle \mathrm{c}$ it is helpful to extend the line $\overline{\mathbf{s c}}$ and to add a line parallel to $\overline{\mathrm{sl}}$ and position it through c. These constructions are shown as dashed lines in figure 5.3(b). We obtain

$$
\angle \mathrm{c}=\pi-\left(\delta_{x}+\theta_{x}-\alpha\right)
$$

The law of sines can now be applied

$$
\frac{\sin \left(\pi-\left(\delta_{x}+\theta_{x}-\alpha\right)\right)}{d_{s}}=\frac{\sin \left(\theta_{x}-\alpha\right)}{d_{c}}
$$

We proceed from equation (5.10) to develop an expression for $\beta$. First we use the fact that $\sin (\pi-a)=\sin a$. This enables us to simplify the left-hand side of equation (5.10) slightly

$$
\begin{array}{r}
\frac{\sin \left(\delta_{x}+\theta_{x}-\alpha\right)}{d_{\mathrm{s}}}=\frac{\sin \left(\theta_{x}-\alpha\right)}{d_{\mathbf{c}}} \\
\frac{d_{\mathbf{s}}}{d_{\mathbf{c}}}=\frac{\sin \left(\delta_{x}+\theta_{x}-\alpha\right)}{\sin \left(\theta_{x}-\alpha\right)}
\end{array}
$$

Next we use the addition rule for sines

$$
\begin{array}{r}
\frac{d_{\mathbf{s}}}{d_{\mathbf{c}}}=\frac{\sin \delta_{x} \cos \left(\theta_{x}-a\right)+\cos \delta_{x} \sin \left(\theta_{x}-a\right)}{\sin \left(\theta_{x}-a\right)} \\
\frac{d_{\mathbf{s}}}{d_{\mathbf{c}}}=\frac{\sin \delta_{x} \frac{\cos \left(\theta_{x}-a\right)}{\cos \left(\theta_{x}-a\right)}+\cos \delta_{x} \frac{\sin \left(\theta_{x}-a\right)}{\cos \left(\theta_{x}-a\right)}}{\frac{\sin \left(\theta_{x}-a\right)}{\cos \left(\theta_{x}-a\right)}} \\
\frac{d_{\mathbf{s}}}{d_{\mathbf{c}}}=\frac{\sin \delta_{x}+\cos \delta_{x} \tan \left(\theta_{x}-a\right)}{\tan \left(\theta_{x}-a\right)}
\end{array}
$$

After a couple of further algebraic steps we obtain

$$
\begin{array}{r}
\tan \left(\theta_{x}-\alpha\right)=\frac{\sin \delta_{x}}{\frac{d_{\mathrm{s}}}{d_{\mathrm{c}}}-\cos \delta_{x}} \\
\theta_{x}-\alpha=\arctan \left(\frac{\sin \delta_{x}}{\frac{d_{\mathrm{s}}}{d_{\mathrm{c}}}-\cos \delta_{x}}\right)
\end{array}
$$

We use the fact that $\beta=\alpha+\pi$ to compute $\beta$

$$
\beta=\theta_{x}-\arctan \left(\frac{\sin \delta_{x}}{\frac{d_{s}}{d_{c}}-\cos \delta_{x}}\right)+\pi
$$

Finally, we include the result of equation (5.8) to obtain the final expression for $\beta$ in terms of $\boldsymbol{\theta}$ and $\boldsymbol{\delta}$

$$
\beta=\theta_{x}-\arctan \left(\frac{\sin \delta_{x}}{\frac{\tan \left(\theta_{y}+\delta_{y}\right)}{\tan \theta_{y}}-\cos \delta_{x}}\right)+\pi
$$


We transform the angle $\beta$ calculated for each correspondence into a home vector $\mathbf{u}$

$$
\mathbf{u}\left(\theta_{x}, \theta_{y}\right)=\left(\begin{array}{l}
\cos \beta \\
\sin \beta
\end{array}\right)
$$

\subsubsection{Computing the Final Home Vector}

If the correspondence vector $\delta$ is correct then the vector $\mathbf{u}$ represents the true home vector. Thus, if it was known that all correspondence vectors were correct, we could simply map any one of these into a home vector and return that home vector as our final answer. However, finding correct correspondences is non-trivial and we assume that some correspondence vectors are incorrect. One possibility would be to use robust estimation techniques such as the RANSAC algorithm to select a maximally consistent set of correspondence vectors [20].

A much simpler strategy is to sum all home vectors together to produce a single final home vector, as in Hong et al.'s technique. As described in section 5.3, summation of home vectors can allow the error components of these home vectors to cancel each other out. For Hong et al.'s technique this was advantageous because the home vectors themselves have error components, even for perfect correspondences. For the technique described above perfect correspondences should lead to perfect home vectors. However, the summation of all home vectors is advantageous for this technique as well.

Correct correspondence vectors will generate correct home vectors-all pointing in approximately the same direction. We will refer to incorrect correspondence vectors as mismatches. If the mismatches are uncorrelated then the home vectors generated from them will also be uncorrelated. Thus, home vectors computed from mismatches will tend to cancel each other out in the summation of home vectors. The main contribution to the final home vector will be from the correct home vectors. Hence the final home vector will be approximately correct. We refer to the assumption that incorrect correspondences are uncorrelated as the uncorrelated mismatches assumption.

Given this assumption, we compute a final home vector by summing all home vector estimates $\mathbf{u}$ together

$$
v=\sum_{i}^{n} u^{i}
$$

where the summation is over all $n$ correspondence vectors. Finally, $\mathbf{v}$ is normalized

$$
\hat{\mathbf{v}}=\frac{\mathrm{v}}{\|\mathbf{v}\|}
$$

The vector $\hat{v}$ above is the final home vector. 


\subsubsection{Correctness}

Note that no approximations were used in the development above. Also, it was not necessary to make any assumptions about the distribution of landmarks in the environment. If given correct correspondence vectors, this technique generates correct home vectors-meaning that the goal can be reached in the smallest possible time. This is certainly an improvement over Hong et al.'s technique. If that technique is given correct correspondence vectors it generates home vectors which are within $\frac{\pi}{2}$ of the true home direction-meaning that the goal can be reached eventually.

\subsection{Comparison}

We compare the methods described above on the example for two-dimensional flow from chapter 2. Recall that this example positioned an agent within a sphere visualized in OpenGL. Markers on the surface of the sphere facilitated finding correspondences between the snapshot image $S$, captured at the centre of the sphere, and the current image $C$. For the coordinate system of this environment, the position $\mathbf{c}$ is to the left of position s. Thus, a correct home vector should be directed to the right.

Figure 5.5(a) shows the correspondence vectors at position c. The intent is to show the performance of various vector mapping techniques on these correct correspondence vectors. However, while these vectors are correct in that matching features are always correctly corresponded, they are not error-free. Some distortion is present due to pixel discretization. Also, the fact that the panoramic images were mapped to a cylindrical, as opposed to spherical projection induces further distortion. However, the effect of these distortions appears to be quite small.

Figure 5.5 shows the home vectors computed by three vector mapping techniques for the correspondence vectors shown in figure 5.5(a). The techniques tested are that of Hong et al. without scaling, Hong et al. with scaling of home vectors by $\delta_{x}$, and finally by the twodimensional technique described in the previous section. The figure captions also indicate the final summed and normalized home vector for each technique. For all techniques, the final home vector is very close to the ideal vector of $(1,0)$. The vectors shown in figures $5.5(\mathrm{~b})$ - (d) are home vectors computed from the correspondence vectors in figure 5.5(a). These home vectors are shown within the image plane at the positions of the correspondence vectors they were mapped from.

A great deal of variation can be observed in the individual home vectors produced by the two variants of Hong et al.'s technique. In particular, near the FOE and FOC the home 
vectors are particularly erroneous. This shows, in part, the wisdom of scaling by $\delta_{x}$. The highest values of $\delta_{x}$ are found away from the foci. This we linow because of the sinusoidal structure of the flow field, as investigated in chapter 2 . It is at these positions, away from the foci, where the home vectors are most correct. The fact that $\hat{\mathbf{v}}_{\text {hong }}$ is close to ideal is due to the cancellation of vector components orthogonal to the true home vector in the summation of equation (5.4). This cancellation occurs because of the isotropic distance assumption which is perfectly valid here. However, if there are particular regions of the flow field that are either absent or erroneous, this cancellation of errors will not occur and the final home vector will be incorrect.

The individual home vectors produced by the two-dimensional technique, however, show very little variation. They all point to the right. This is highly advantageous as it means that the vector mapping stage of visual homing need not be a source of error. Further, even if we have correspondence vectors from only a very small portion of the flow field, these vectors can still generate correct home vectors. With Hong et al.'s technique correspondence vectors from regions near the foci will produce incorrect home vectors.

One issue with the two-dimensional vector mapping technique is that correspondence vectors along the horizon are unusable because of division by zero in equation (5.8). However, this problem occurs only at the precise location of the horizon. Another issue is the difference in complexity. For the two-dimensional we must evaluate equation (5.19) to obtain the direction of individual home vectors, while for Hong et al.'s technique equation (5.3) is required. Certainly equation (5.19) is more complex, but these are both closed-form expressions which can be easily computed (lookup tables could be employed for equation (5.19) if need be). 


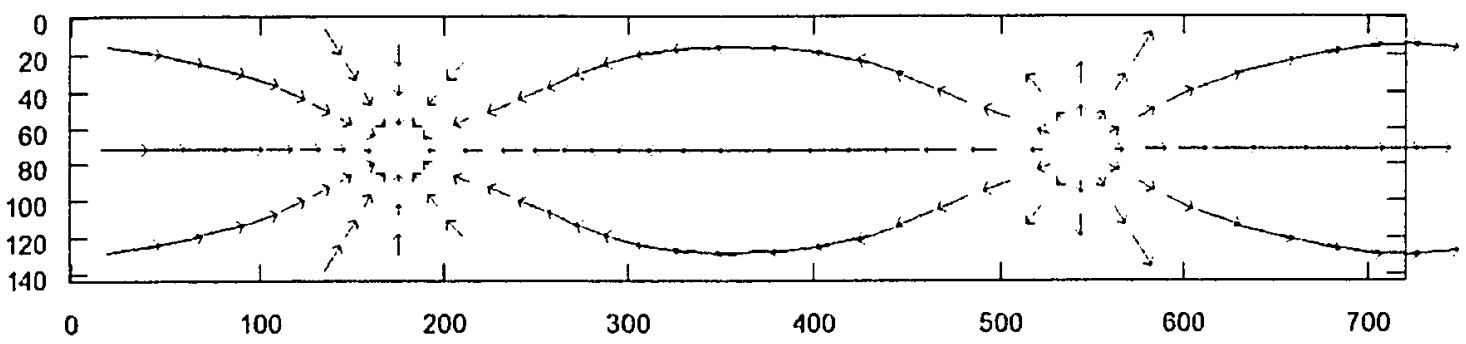

(a) Correspondence Vectors

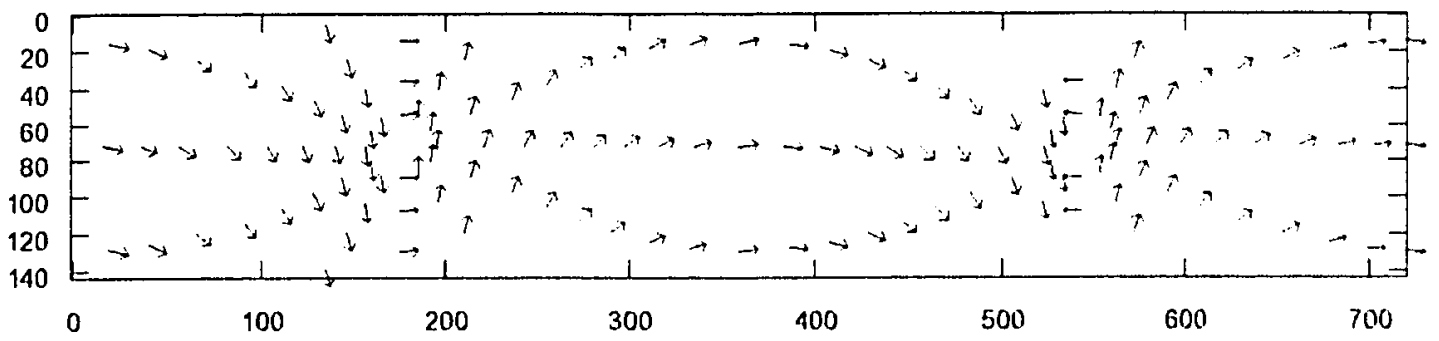

(b) Hong: $\hat{\mathrm{v}}_{\text {long }}=(0.99996,-0.00869)$
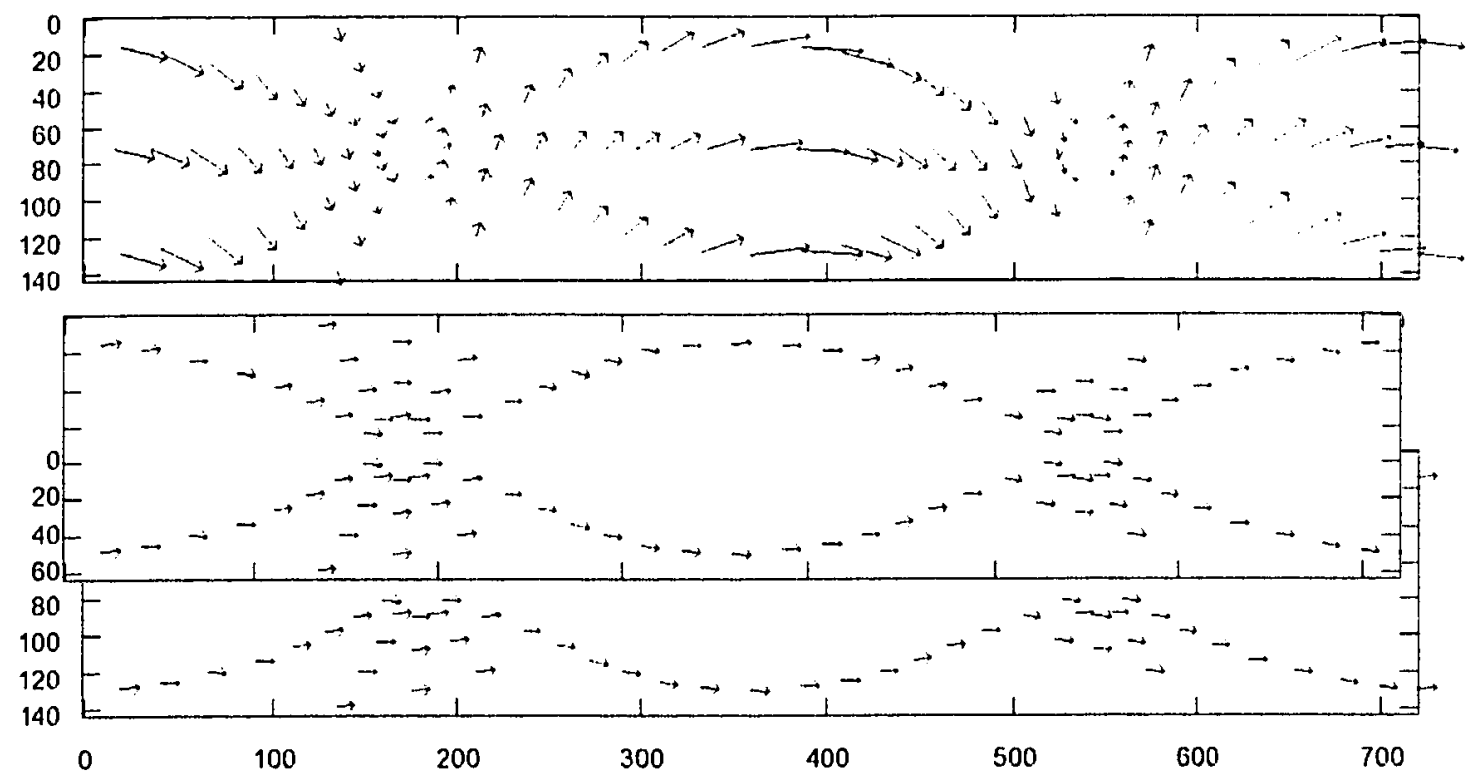

(d) 2-D: $\hat{\mathrm{v}}=(0.99999,-0.00173)$

Figure 5.5: Performance of three techniques for visual homing for the sphere environment introduced in chapter 2. 


\section{Chapter 6}

\section{CGSM}

\subsection{Introduction}

This chapter presents a homing method employing an imovative technique for feature correspondence. This technique allows correspondences to be established in adherence with the local processing constraints of a retinotopic matrix. Earlier variants of this method have been published in [99] and [100].

The method's feature correspondence technique grows a potential surface around feature points extracted from one image. Then, the gradient of this surface is determined at the position of features in the other image. In this manner, correspondence vectors relating features are generated. Mapping of correspondence vectors into home vectors occurs through the vector mapping scheme of chapter 5 . The features extracted by this method are Harris corners [37]. As the features are corners, and the method for corresponding them involves finding the gradient of a potential surface, we shall refer to this method as the Corner Gradient Snapshot Method' ${ }^{1}$ or CGSM.

CGSM is an extension of Möller et al.'s [76, 77] neural snapshot model. Möller et al.'s method introduced the idea of using the gradient of a potential surface to determine the direction of feature correspondence. That work applied to one-dimensional images of a two-dimensional simulated world. Hence, the potential surface was also one-dimensional. CGSM extends the neural snapshot model to two-dimensional images and a two-dimensional potential surface. CGSM extends Möller et al.'s work in other was as well. It operates on

\footnotetext{
${ }^{1}$ In [99] and [100] this method was referred to as the Corner Gradient Snapshot. Model. However, the homing methods presented in this thesis should not be considered models. Therefore the word 'Method' is substituted for 'Model'.
} 
images of the real world, as opposed to simulated images. It encodes not only the direction but also the distance of correspondence. This is achieved by using the technique of Hassoun and Sanghvi [40] to generate the potential surface ${ }^{2}$. The encoding of distance in the potential surface allows the exact vector mapping technique of chapter 5 to be used, as opposed to the approximate technique used by Möller et al.

In addition to explaining CGSM itself, this chapter presents detailed wiring diagrams to illustrate how some of its operations are implemented within a retinotopic matrix. Subsequent chapters will utilize some of the same operations as well as others, but will not generally include detailed wiring diagrams as are presented here. The purpose of these diagrams is to exemplify how various operations can be achieved in a retinotopic matrix.

\subsection{Assumption}

As in the original snapshot model, CGSM corresponds image features based only on proximity. A feature in the snapshot image is always paired with the closest feature in the current image. These correspondences are only guaranteed to be correct if the agent's displacement is small, and the same features remain detected between the snapshot and current images. Where the agent's displacement is not small and there is variability in feature detection, CGSM relies on the uncorrelated mismatches assumption, described in section 5.4.1.

\subsection{Overview of Processing}

Figure 6.1 depicts the processing applied by CGSM. Figure 6.1(a) depicts that part of the processing which is applied to both snapshot and current images. For this reason, the input image is denoted $X$ and the output image is denoted FcaturcX. A Gaussian filter is first applied to prepare for corner detection. Then the Harris comer detector is applied. This detector responds to pixels of the Gaussian-filtered image according to their quality as corners. The result is a 'hilly corner image'. Another Gaussian filter then blurs this image so that immediately adjacent 'hills' of moderate activity become singular 'mountains' of great activity. The peaks of these 'mountains' are then extracted by suppressing all points which are not local maxima. Only those peaks whose height exceeds a certain threshold are subsequently extracted as the final features in image Feature $X$. If processing the snapshot image $S$ then Feature $S$ represents the final stored image.

\footnotetext{
2The use of Hassoun and Sanghvi's method was suggested by Ralf Möller as an alternative to the method employed in [99].
} 

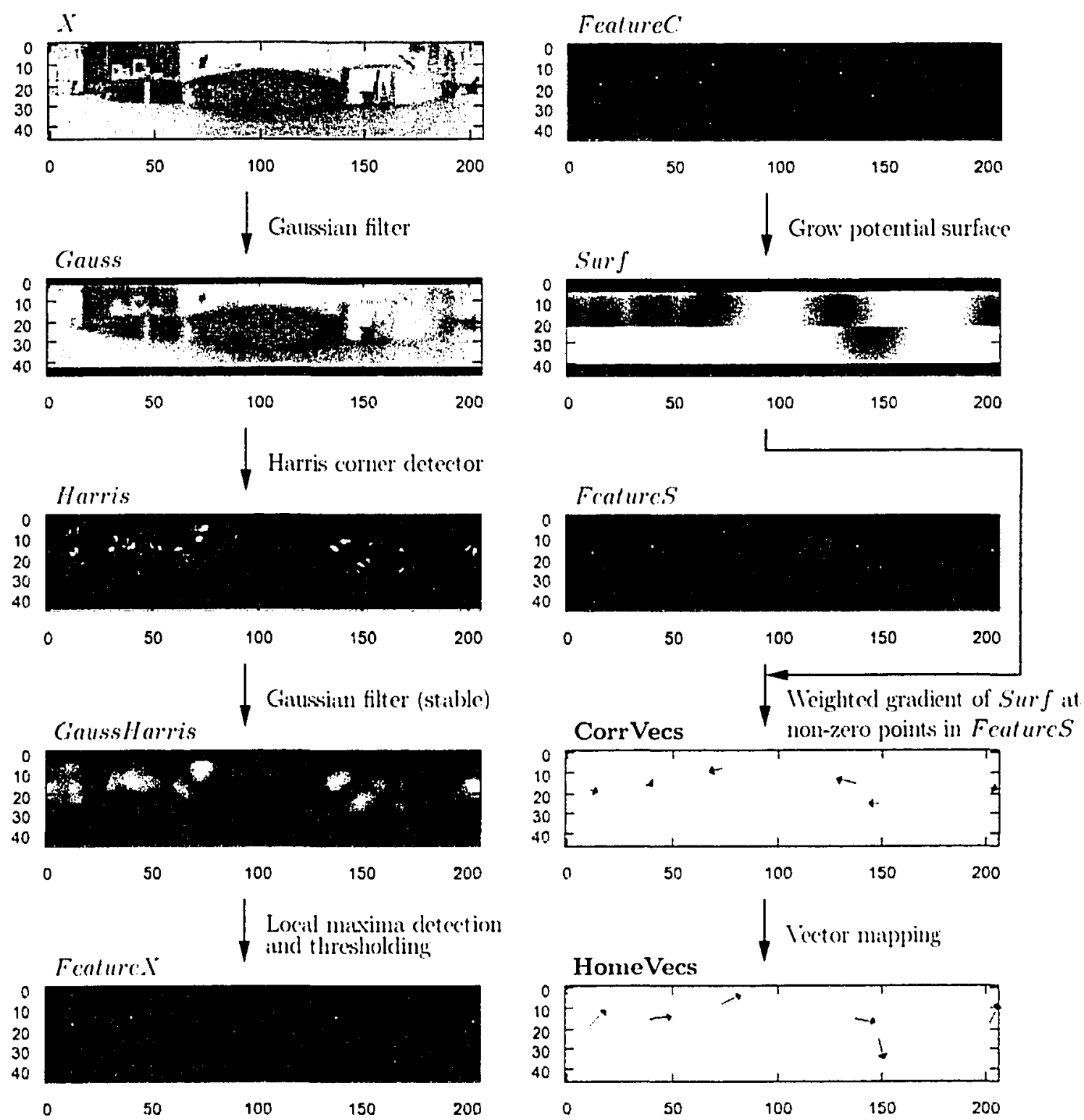

(a) Processing image $X^{*}(S$ or $C$ )

(b) Processing image $C$.

Figure 6.1: Processing applied by CGSM. The snapshot image is from position $(3,14)$ of image collection original and the current image is from position $(1,14)$. The vectors in CorrVecs are shown at the appropriate lengths, while the unit vectors in HomeVecs have been scaled by 10 for visibility. All of the non-vector images have been rescaled such that their maximal values are represented in white. 
The processing for the current image is divided into two stages. The first stage is identical to that described above, with $C$ replacing $X$. The second stage is depicted in figure 6.1(b). FeatureC is the input to this second stage. The non-zero points in this image serve as source points from which to grow the potential surface image Surf. Next, weighted gradients of Surf are found at non-zero points in FeatureS. The gradient is a two-dimensional vector that gives the 'uphill' direction of the potential surface. These gradient vectors are weighted in such a way that they yield correspondence vectors. These correspondence vectors are placed in the image pair CorrVecs (vectors are represented by multiple images, one for each dimension). Next, correspondence vectors are mapped into home vectors, HomeVecs, via the vector mapping method of chapter 5. The vectors in HomeVecs are then summed and normalized to yield a single home vector. The correct home vector for the images in figure 6.1 is directed to the right. The normalized sum of HomeVecs in this figure is directed approximately to the right: $(0.973,-0.231)$.

The operations depicted in figure 6.1 will be further elaborated upon below. First, however, the framework for describing and implementing these operations in a retinotopic matrix is presented.

\subsection{Describing a Retinotopic Matrix}

A retinotopic matrix can be described by giving the neighbourhood and function of each processing element along a column through the matrix. The neighbourhood of a processing element describes which of its peers it receives input from as well as any weights associated with those inputs. The function achieved by each processing element is one of the following:

Conv The sum of all neighbours' weighted activation levels is returned. This is the convolution operation, prevalent in signal processing.

Mult The product of all neighbours' weighted activation levels is returned.

IsMax If the central neighbour has the highest weighted activation of all neighbours then 1 is returned. Otherwise 0 is returned. The central neighbour has the same image coordinates as the processing element.

Thold $(f)$ If the central neighbour's weighted activation level is above $f$ then 1 is returned. Otherwise 0.

Pow $(f)$ The central neighbour's weighted activation level is raised to power $f$. 
Expr Each neighbour may have a symbol associated with it whose purpose is to stand for that neighbour's activation level in an algebraic expression. The algebraic expression can include any of the usual arithmetic operators and common numerical functions $(+,-, *, /,-$, sin, cos, sqrt, and the constant pi $)$. Also, the following special symbols are used to stand for certain element-specific parameters or global constants:

$c$ columm index of processing element in image (i.e. $x$-coordinate)

$r$ row index of processing element in image (i.e. $y$-coordinate)

$w$ image width

$h$ image height

An element applies its function and then takes on the resultant value. All elements on a single layer are updated synchronously. In this implementation images are horizontally panoramic but vertically bounded. If an element's neighbourhood stretches horizontally' past the image boundary then it is wrapped around toroidally to the opposite side of the image. If an element's neighbourhood stretches vertically past the image boundary then the element will be given a special out-of-bounds value, causing any subsequent elements that connect to this element to also become out-of-bounds. Effectively the image being processed will shrink vertically: This approach allows all functions to handle the border in a uniform manner.

\subsection{CGSM}

\subsubsection{Gaussian Filter}

The Harris corner detector can be sensitive to high-frequency noise. Therefore, a Gaussian filter is applied directly on the input image in order to attenuate this noise. The kernel for this filter is the same as that presented in section 4.2.4. This kernel is separable, meaning that it can be split into two identical, but orthogonal layers [96]. Figure 6.2 shows how the Gaussian filter can be implemented in a retinotopic matrix. Weights between the input image layer and the middle layer are given by the kernel, applied in the horizontal direction. The same weights applied in the vertical direction from the middle image yield the filtered image Gauss. 


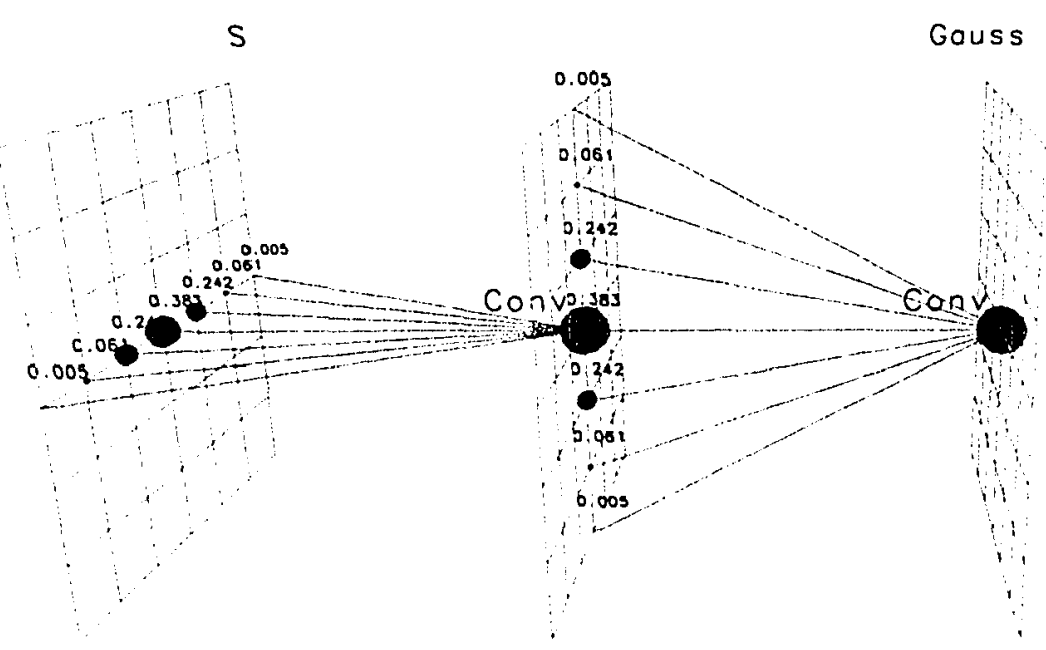

Figure 6.2: Implementation of Gaussian filter in a retinotopic matrix. The green spheres are processing elements. The neighbourhood of each processing element is indicated by connections to blue splieres. The radius of the blue spheres is proportional to the strength of connection. Labels above the blue spheres give the connection weights (no label indicates a connection weight of 1 ).

\subsubsection{Harris Corner Detector}

We briefly review the technique used for corner detection before describing how it is implemented in CGSM. The technique is known as the Harris corner detector [96] (pages 82-85) and was originally presented in [37]. Consider an image point, p. and a small window centred on $\mathbf{p}$. If the window is shifted then we can compare the old and new windows and describe the amount of change that has occurred as a result of the shift. A corner is defined as an image point where this degree of change is large for all possible slifts. A simplified check for this conclition is that, for the shift producing the smallest change, the level of change must still be large. An analysis is presented in [37] which cquates this minimum change with the smaller eigenvalue of matrix $M$,

$$
\mathbf{M}=\left[\begin{array}{cc}
I & K \\
K & J
\end{array}\right] \quad \text { where } I=\sum D_{x}^{2} . \quad J=\sum D_{y}^{2} . \quad K=\sum D_{x} D_{y}
$$

where $D_{x}$ and $D_{y}$ are the spatial derivative images of Gauss. The kernels used to approximate the spatial derivatives of an image in the $x$ and $y$ directions are $\left(\begin{array}{lll}1 & 0 & -1\end{array}\right)$ and $\left(\begin{array}{lll}1 & 0 & -1\end{array}\right)^{T}$. Convolution by these kernels implements the central-difference approximation of the first derivative (see Appendix A.2 of [96]). The summations above cover pixels in a 
small window of radius $r$, centred on $\mathbf{p}$.

After a few algebraic steps we can arrive at an equation for the smaller eigenvalue, denoted $\lambda_{s}$,

$$
\lambda_{s}=\frac{I+J-\sqrt{(I+J)^{2}-4\left(I J-I^{2}\right)}}{2}
$$

$\lambda_{s}$ will be the output from the Harris corner detector as implemented here. This quantity responds to pixels of the Gaussian-filtered image according to their quality as corners.

The wiring diagram to implement the Harris corner detector is somewhat intricate. Rather than present the whole diagram, figure 6.3 presents only that portion of the retinotopic matrix which computes $I$.

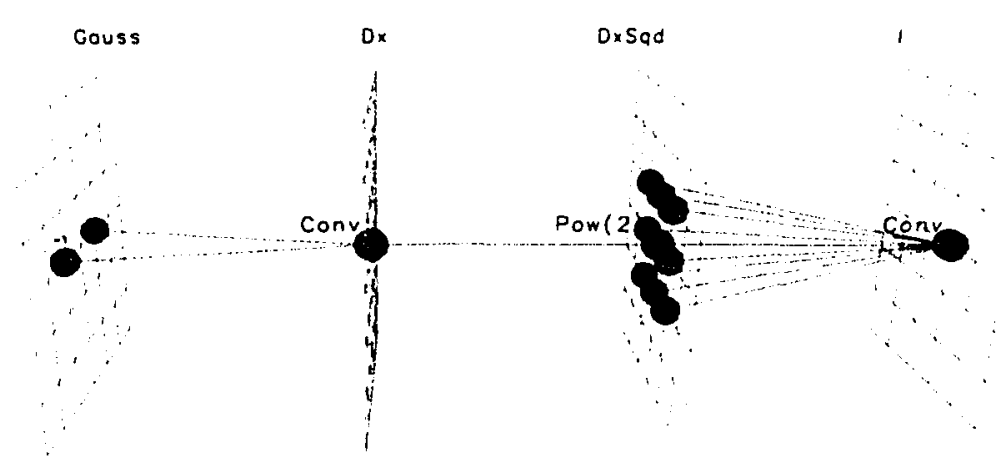

Figure 6.3: Part of the implementation of the Harris corner detector in a retinotopic matrix. Only the computation of $I$ is shown. See figure 6.2 for notation. The red sphere indicates a negative connection weight.

\subsubsection{Gaussian Filter (Stable)}

The output of the Harris corner detector' is the 'hilly corner image' Harris. Each pixel of this image gives the quality of corner at the corresponding pixel in Crauss. In the version of the Harris corner detector described by Trucco and Verri [96]. the values in Harris would next be sorted according to value. The sorted list of corners would then be filtered to remove weaker corners which were within radius $r$ of stronger corners. Each of the surviving corners is made more distinctive by being the only corner within radius $r$.

However, the approach of Truces and Verri is not feasible within a retinotopic matrix. 'To achieve a similar effect, a Ganssian filter is applied repeatedly to Harris. Whereas in Harris a number of local minima may have existed within a small neighbourhood, the filtered 
image Gaussharris will have fewer local maxima ${ }^{3}$. These local maxima are therefore more distinctive. Unfortunately, repeated applications of our normal Gaussian filter cause the image to shrink vertically. This is because the neighbourhood of the Gaussian can overlap the top and bottom boundaries of the image where there are no valid pixels. Normally: this causes the output of that Gaussian to be set to out-of-bounds-therefore shrinking the image. To prevent this the invalid out-of-bounds inputs can be treated as zeroes. We refer to this altered mamner of applying the Gaussian as stable.

\subsubsection{Local Maxima Detection and Thresholding}

The surviving local maxima in Ganss Harris must be extracted as discrete features. Figure 6.4 shows how this is achieved through the IsMax function on image GaussHarris. We also wish to retain only those features whose activity is above a particular threshold. This is achieved through the Thold function, which is also applied to image GaussHarris. Both IsMax and Thold return either 0 or 1 . Thus, the images from the output of these two functions can be combined in a logical AND by multiplying them together. Figure 6.4 shows how this is achieved through the Mult function. After this segment of the retinotopic matrix is applied we are left with FeatureS, the image of discrete features from $S$.

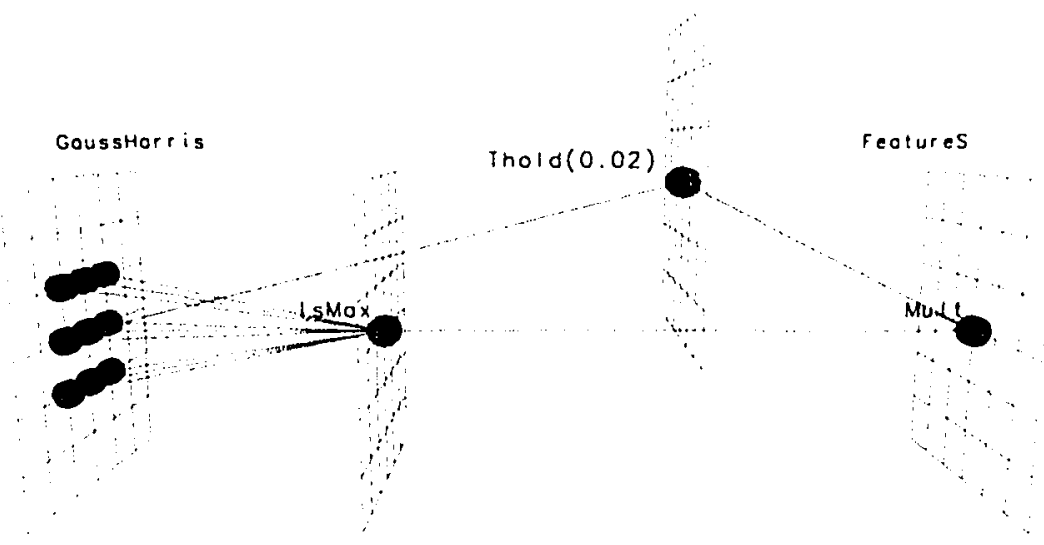

Figure 6.4: Implementation of local maxima detection and thresholding in a retinotopic matrix. The third layer is in a raised position only to clarify the comnectivity between layers. See figure 6.2 for notation.

\footnotetext{
${ }^{3}$ Scale-space theory reveals that repeated application of a Gaussian filter can only reduce the number of local maxima [64].
} 


\subsubsection{The Potential Surface}

A potential surface is an image whose activity at each pixel is proportional to the cost of travelling from that pixel to the nearest source point. A potential surface is naturally divided up into regions called wells, where the activity of pixels within a well give the cost of travelling to that well's source point ${ }^{4}$. Here we shall equate this cost with distance in image coordinates. The source points for CGSM are the active pixels in FeatureC. The fact that the distance to source points is encoded in the potential surface is important. Perhaps more important is that the negative of gradient of the potential surface gives the direction of the nearest source point. This is the feature which CGSM exploits to determine correspondence vectors within a retinotopic matrix.

Hassoun and Sanghri's [40] technique is used to grow the potential surface from its source points. The intended goal of their algorithm is to determine the optimal path between two points on a grid, and to compute this path with a set of processors which are spatially distributed on the same grid ${ }^{5}$. To this end, the first stage of their algorithm distributively computes a potential surface whose height gives the cost of moving to the closest source point. Only this aspect of their algorithm is used here.

The potential surface image $\operatorname{Sur} f$ is initialized to 0 at positions which are active in Features $C$, and to an arbitrarily large value $v_{p s}$ everywhere else. The potential surface is developed through a recursive update rule, applied for a fixed number of iterations, $n_{p s}$. The update rule is recursive in that the current potential surface Surf is used to create the new surface $\operatorname{Sur} f^{\prime}$. The current activity of a potential surface pixel is given by $\operatorname{Sur} f(x, y)$. The set of neighbours of $(x, y)$ is $\Pi_{x, y}$. The distance from $(x, y)$ 's neighbour $(p, q)$ to $(x, y)$ is $d_{p, q}$. The recursive update rule is as follows:

$$
\operatorname{Sur} f(x, y)^{\prime}=\min \left\{\operatorname{Sur} f(x, y), \min _{(p, q) \in \Pi_{x, y}}\left(\operatorname{Sur} f(p, q)+d_{p, q}\right)\right\}
$$

It is convenient to initialize non-source points on the potential surface with the number of iterations, $n_{p s}$. This means that $\operatorname{Sur} f$ begins with all non-source pixels at value $n_{p s}$, and all source pixels at 0 . After $n_{p s}$ iterations there will be wells on the surface centred on the source pixels which increase smoothly to plateau out at $n_{p s}$. Figure 6.5 illustrates the development of the potential surface from a small source image over five iterations.

The number of iterations for figure 6.5 was set to 10 , yet the potential surface actually converged by the fifth iteration. This convergence is premature as there remain a number of pixels still at their initial value of $v_{p s}=10$. In fact, it is the setting of $v_{p s}=n_{p s}$ which

\footnotetext{
${ }^{4}$ The divisions between wells of a potential surface form a Voronoi diagram (see chapter 7 of $[14]$ ).

"A device-efficient hardware implementation of this same idea is presented in [71].
} 
FealureC

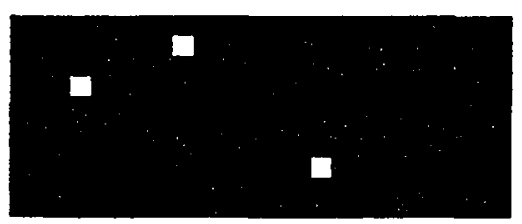

iteration 0

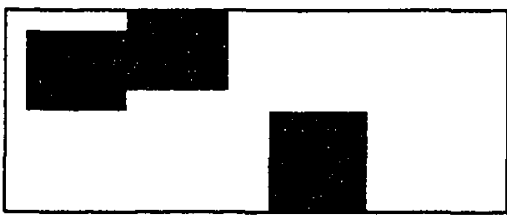

iteration 1

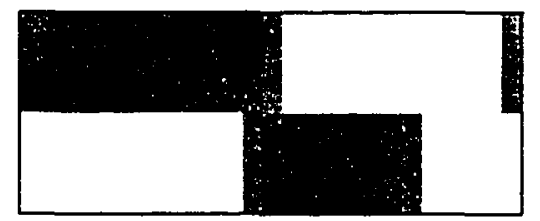

iteration 2

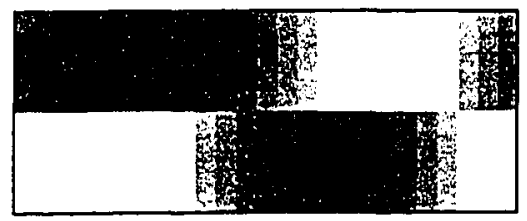

iteration 3

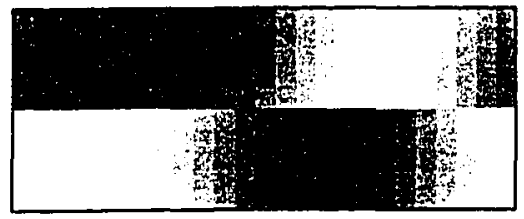

iterations $4-\infty$

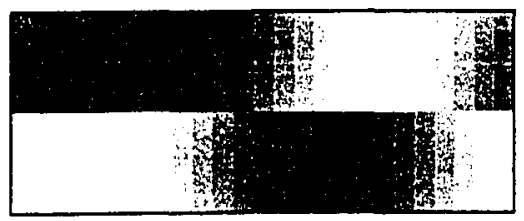

Figure 6.5: Development of potential surface over five iterations with $n_{p s}=v_{p s}=10$. For the potential surface images, white corresponds to 10 .

has caused the premature convergence. Initializing the potential surface to 10 means that the potential wells around each source-point can grow only up to a maximum radius of 10 . If we instead set $v_{p s}=\infty$ then these wells could grow until all pixels had been set to a value less than infinity. However, this is a computationally expensive operation and the number of iterations should be kept low. Setting $v_{p s}=n_{p s}$ allows convergence to occur within a fixed distance from source points.

Figure 6.5 also illustrates that, in fact, two completely separate potential surfaces are developed: one above the horizon and one below. The purpose of this split is to prevent features from the top half of the image from being corresponded with those in the bottom half, and vice versa. For an agent translating in the plane, no image feature should cross the horizon. The horizon of a panoramic image is the line which does not undergo vertical translations under movements of the robot in the plane. For the Bielefeld image clatabase the horizon occurs at about the halfway vertical position. Splitting the potential surface 
at the horizon prevents spurious correspondences from being made between the two image halves.

\subsubsection{The Weighted Gradient of the Potential Surface}

At any point on the potential surface the direction that is most directly 'downhill' points towards the nearest source point. Mathematically; this direction is given by the negative of the gradient. The gradient of an image $\mathrm{X}$, denoted $\nabla X$, is defined as follows:

$$
\nabla X=\left(\begin{array}{l}
\frac{\partial x}{\partial x} \\
\frac{\partial x}{\partial y}
\end{array}\right)
$$

The partial derivatives above can be estimated by convolution of the image $X$ with the kernels $\left(\left(\begin{array}{lll}1 & 0 & -1\end{array}\right)\right.$ and $\left(\begin{array}{lll}1 & 0 & -1\end{array}\right)^{T}$ (we first met these in chapter 6$)$.

While the direction to the nearest source point is given by $-\nabla \operatorname{Sur} f$, the distance is not. However, this distance is already encoded in the value of the potential surface. We can create a vector with the appropriate distance and direction by nomalizing $-\nabla S u r f$ and multiplying it by $\operatorname{Sur} f(x, y)$,

$$
-\operatorname{Sur} f(x, y) \frac{\nabla \operatorname{Sur} f(x, y)}{\|\nabla \operatorname{Sur} f(x, y)\|}
$$

This vector gives the approximate direction and distance from $(x, y)$ to the nearest source point. However, only those vectors at positions of detected features in the snapshot image are correspondence vectors. Other vectors can be excluded by multiplication with the binary image Features. Combining all of this, the correspondence vector $\delta$ for inage position $(x, y)$ is obtained as follows:

$$
\delta=-F \text { eatureS }(x, y) \operatorname{Sur} f(x, y)\left(\begin{array}{cc}
\Delta_{x} & 0 \\
0 & \Delta_{y}
\end{array}\right) \frac{\nabla \operatorname{Sur} f(x, y)}{\|\nabla \operatorname{Sur} f(x, y)\|}
$$

where $\Delta_{x}$ is the horizontal inter-pixel angle and $\Delta_{y}$ is the vertical inter-pixel angle. These multipliers are required so that $\delta$ is expressed as a pair of angles.

\subsubsection{Calculating the Home Vector}

We now have a set of correspondence vectors which ideally describe the movement of features in $S$ to their new positions in $C$. From each of these correspondence vectors we can determine a home vector using the exact vector mapping method of chapter 5 . The final home vector is generated by summing all home vectors together and normalizing. 


\subsubsection{Order of Matching}

The version of CGSM presented above spends more computational effort in processing the current image than the snapshot image. This is because we have followed the paradigm of finding where snapshot image features have travelled to in the current image. However, the reverse-finding where current image features have travelled from in the snapshot imageis equally valid. Adopting this reversed paradigm allows the computational burden to be shifted from the current image to the snapshot image. This is preferable as an agent will tend to spend far more time homing in towards a goal than sitting at the goal capturing and processing the snapshot image.

Figure 6.6 illustrates the processing applied by reversed CGSM. The processing required to arrive at the inages FeatureS and FeatureC is still the same as was presented in figure 6.1(a). The potential surface is now developed from features in the snapshot image, FeatureS. This has a big impact on the computational cost, as developing the potential surface is CGSM's most expensive operation. Next, the weighted gradient is found for all points on $\operatorname{Sur} f$. This generates the image pair CorrVecs which contains all possible correspondence vectors from current image features to snapshot image features. An important proviso is that the vectors in CorrVecs will be of length zero wherever the potential surface is flat. The next step is to apply vector mapping to all vectors in CorrVecs. To account for the reversal of matching, the resultant home vectors must be multiplied by -1 . Finally; the image pair HomeVecsS is stored.

Processing of the current image is now much simpler. First, the features of the current image are extracted into FeatureC. Then vectors from the stored image pair HomeVecsS that are at non-zero positions in Feature $C$ are selected and placed in HomeVecsC. This is simply achieved by multiplying the binary image FcatureC with HomeVecsS. The final home vector is computed by summing the vectors in HomeVecsC and normalizing the result.

\subsection{Optimizing Parameters}

The parameters tested for CGSM are as follows:

- Radius of neighbourhood scimned by Harris corner detector: 1, 2, or 3.

- Number of applications of the Gaussian low-pass filter on the output of the Harris corner detector: 1,3 , or $\mathbf{5}$. 

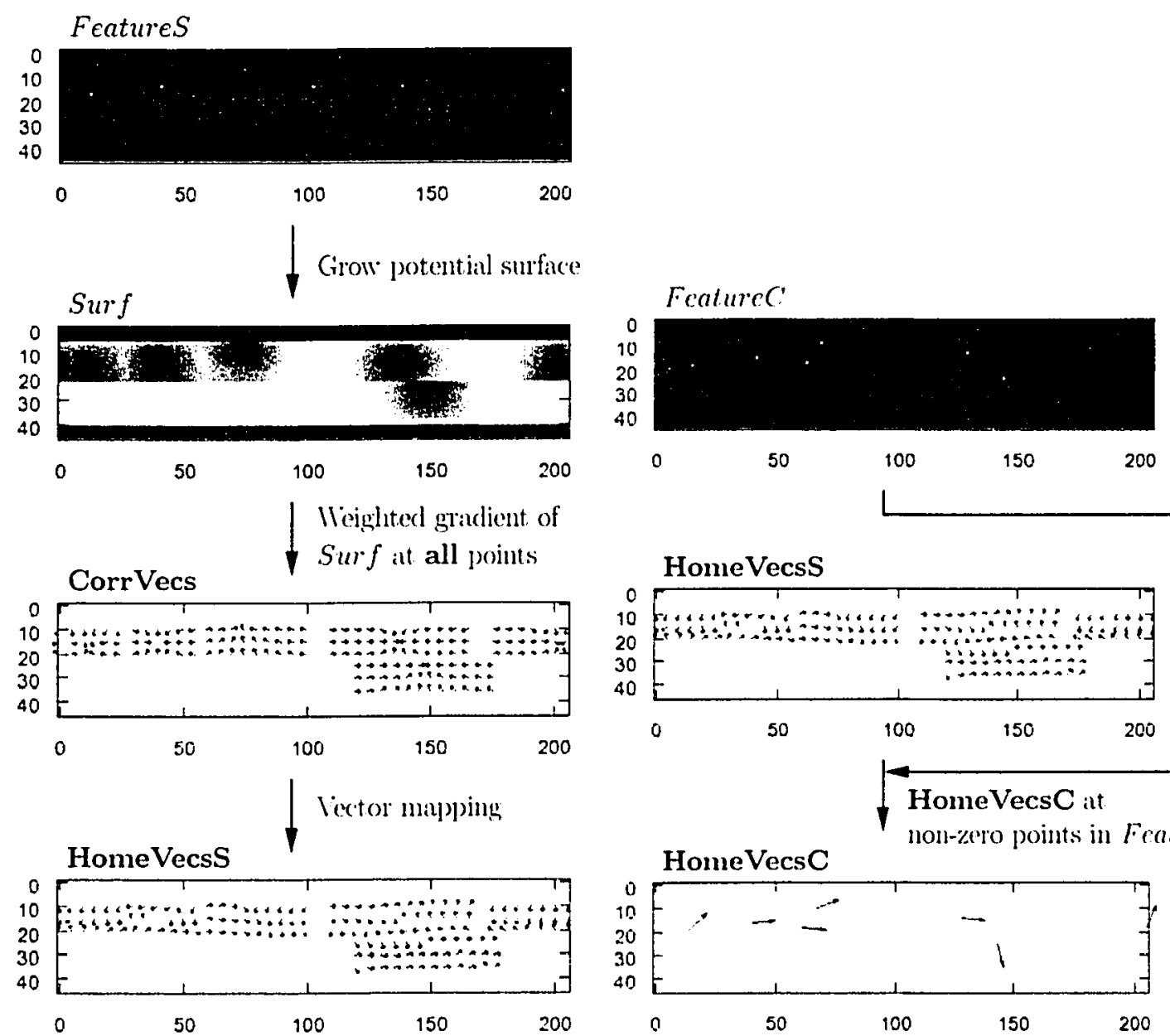

(a) Processing image $S$

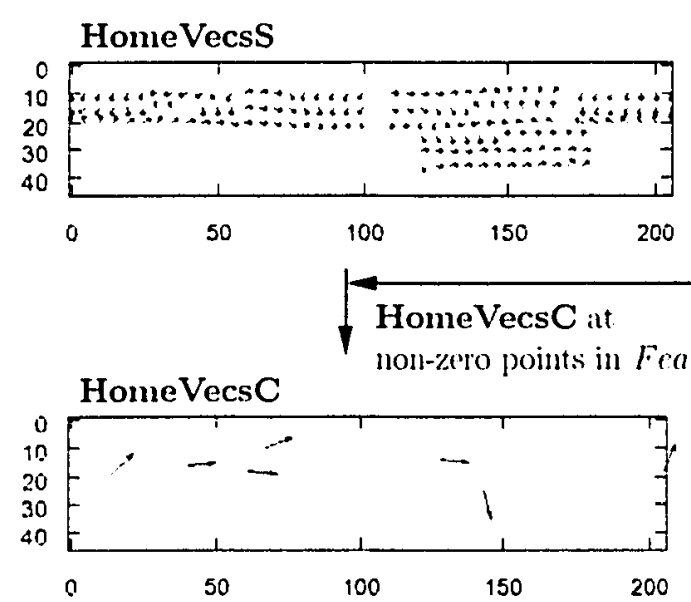

(b) Processing image $C$

Figure 6.6: Processing applied by reversed CGSM. The choice of snapshot and current images is the same as figure 6.1. For CorrVecs and HomeVecsS only every fifth vector is shown. The vectors in CorrVecs have varying lengths but are shown at length 5 for visibility. Similarly, the unit vectors in HomeVecsS have been scaled to length 5, while those in HomeVecsC have been scaled to length 10. 
- Threshold for filtered corner image point to be maintained as a feature: $0.005,0.01$, $0.02,0.03,0.04,0.05$, or 0.1 .

- Number of iterations applied for growth of potential surface: 10,30 .

The best combination found for the current-to-snapshot (i.e. reversed) matching order is given above. The same parameter combination was tested for the snapshot-to-current matching order, which was found to perform slightly worse. No further investigation into the performance difference between these two variants was carried out. Such investigation was felt to be unnecessary as the current-to-snapshot variant is inherently more efficient and there is no reason to suspect any great difference in performance between the two. Therefore, all subsequent references to CGSM shall imply the current-to-snapshot variant.

\subsection{Results on Image Database}

\section{Image Collection original}

Figure 6.7 shows home vectors generated by Warping and CGSM on image collection original for snapshot positions $(6,4)$ and $(0,16)$. For snapshot position $(6,4)$ Warping's home vector field is clearly superior, however CGSM's vector field is also reasonably good-the high value of $R R(0.923)$ reflects this. However, for snapshot position $(0,16)$ it is CGSM's vector field which is superior and both performance metrics reflect this (see figure captions).

Figure 6.8 plots $A A E$ and $R R$ over twenty different snapshot positions. The performance of CGSM is relatively constant in both $A A E$ and $R R$ except for the upper-right comer of the plots which shows the highest error. The reason for poor performance in this region will be explained below.

\section{Image Collections hall1 and hall2}

Figure 6.9 shows home vectors generated by warping and CGSM on image collections hall1 and hall2 for snapshot positions $(3,12)$. Here the results are rather more lopsidedly in favour of Warping. The home vector fields of CGSM are, again, moderately successful for both hall1 and hall2. However, those for Warping are clearly superior.

The superior performance of Warping over CGSM is reflected also in the plots of multiple snapshot positions, shown in figure 6.10 for hall1 and figure 6.11 for hall2. In comparison to Warping, the plots for CGSM are generally more erratic. 


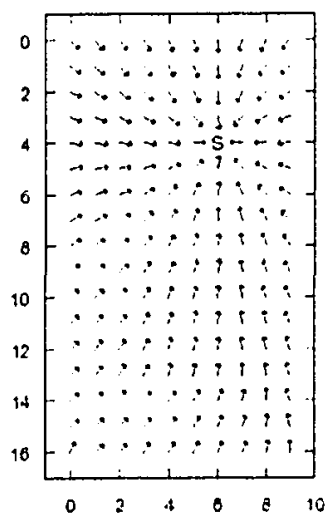

(a) Warping, $\mathrm{s}=(6,4)$ : $A . A E=0.119$ $R R=1.000$

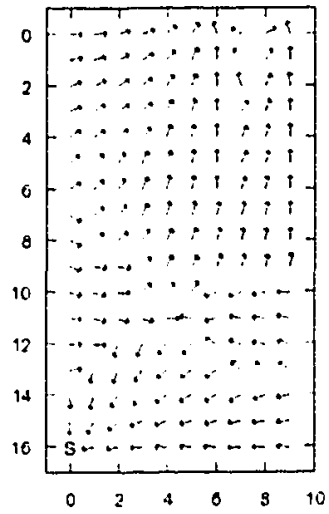

(b) Warping, $\mathrm{s}=(0,16)$ : $A A E=1.758$ $R R=0.266$

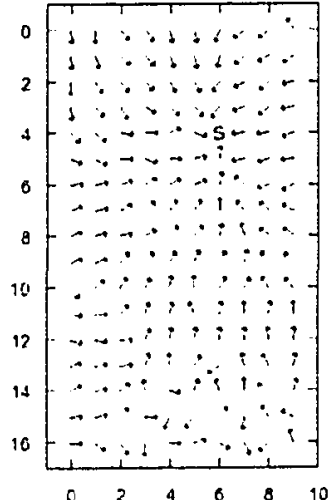

(c) CGSM, $s=(6,4):$ $A .4 E=0.562$, $R R=0.923$

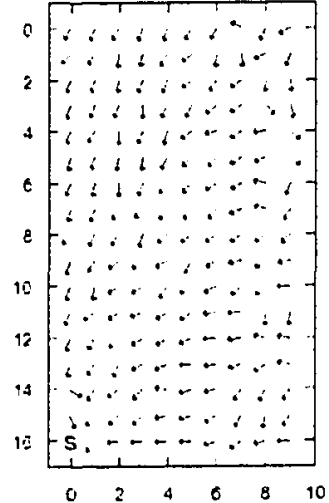

(d) CGSM, $s=(0,16):$ $A A E=0.417$, $R R=0.893$

Figure 6.T: Home vector fields for Warping (a,b) and CGSM (c,d) for snapshot positions $(6,4)(a, c)$ and $(0,16)(b, d)$ in image collection original.

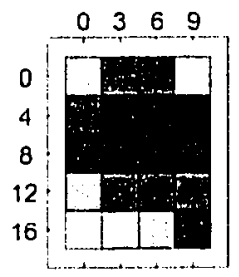

(a) Warping: $A A E_{*}=$ $0.576 \pm 0.461$

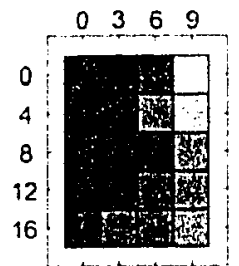

(b) CGSM: $A A E_{*}=$ $0.517 \pm 0.22$

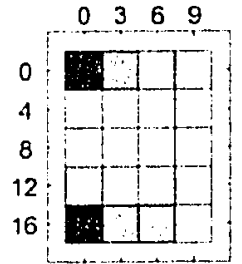

(c) Warping: $R R_{\star}=$ $0.79^{\circ} \pm 0.226$

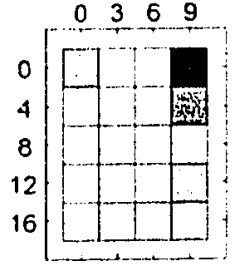

(d) CGSM: $R R_{*}=$ $0.834 \pm 0.229$

Figure 6.8: $A A E$ and $R R$ across snapshot positions for all methods on image collection original. Beneath each plot is shown the mean value of the metric $\left(A A E_{*}\right.$ or $\left.R R_{*}\right)$ and its standard deviation (the number following the ' \pm ' symbol). Average values for $A A E \max .=1.758, R R \max .=1$. 


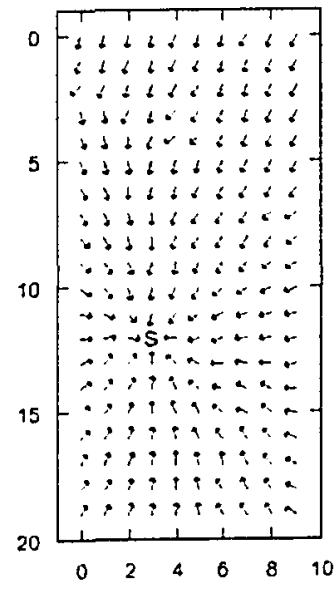

(a) Warping,

hall1, $\mathbf{s}=(3,12)$ : $A A E=0.160$, $R R=1.000$

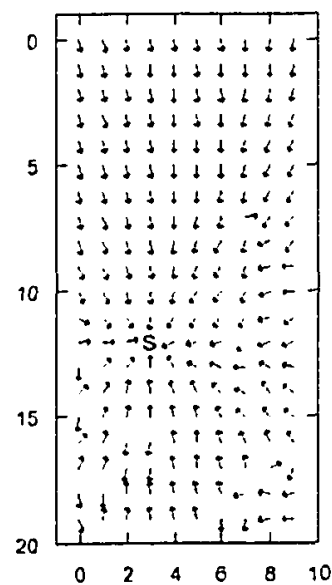

(b) Warping, hall2, $\mathrm{s}=(3,12)$ : $A A E=0.40 \overline{7}$, $R R=0.915$

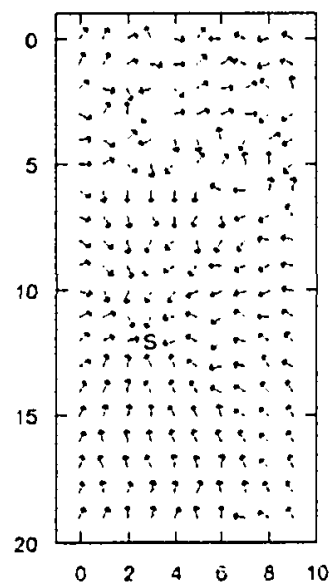

(c) CGSM,

hall1, $\mathrm{s}=(3,12):$ $A A E=0.786$, $R R=0.709$

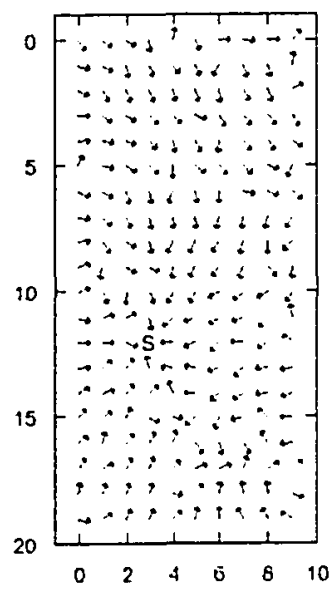

(d) CGSM, hall2, $\mathbf{s}=(3,12)$ : $A . A E=0.774$, $R R=0.714$

Figure 6.9: Home vector fields for Warping (a,b) and CGSM (c,d), for snapshot position $(3,12)$ in image collections hall1 and hall2.

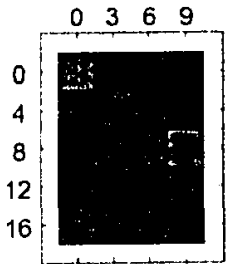

(a) Warping: $A A E_{+}=$ $0.295 \pm 0.151$

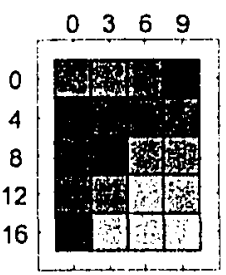

(b) CGSM: $A A E_{*}=$ $0.75 \pm 0.288$

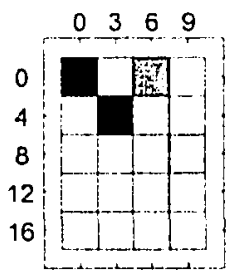

(c) Warping: $R R_{*}=$ $0.841 \pm 0.289$

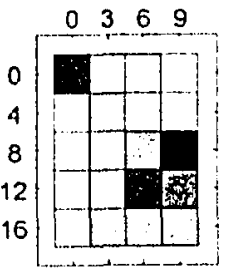

(d) CGSM: $R R_{\star}=$ $0.67 \tau \pm 0.259$

Figure 6.10: $A A E$ and $R R$ across snapshot positions for all methods on image collection hall1. $A A E$ max. $=2.288, R R$ max. $=1$. 


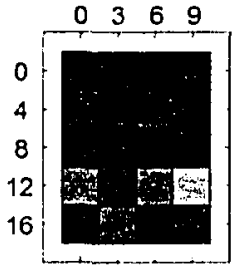

(a) Warping:

$A A E_{*}=$

$0.45 \bar{\top} \pm 0.185$

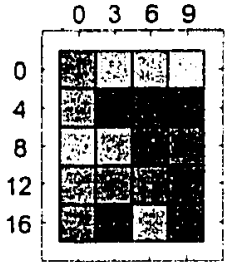

(b) CGSM:

$A A E,=$

$0.761 \pm 0.205$

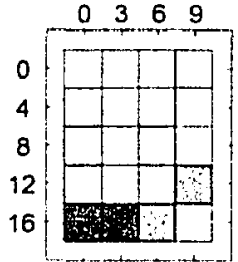

(c) Warping:

$R R .=$

$0.798 \pm 0.202$

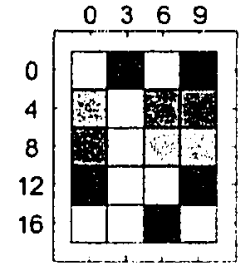

(d) CGSM:

$R R_{*}=$

$0.534 \pm 0.261$

Figure 6.11: $A A E$ and $R R$ across snapshot positions for all methods on image collection hal12. $A A E$ max. $=2.127, R R$ max. $=1$.

\section{Cross-Collection Tests}

Figure 6.12 plots $A A E_{*}$ and $R R_{*}$ for the cross-collection experiment. CGSM exhibits relatively low variation in $A A E$. However, in $R R$, its performance is again rather erratic.

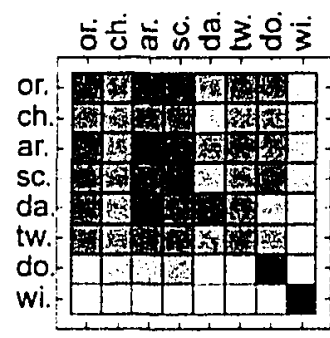

(a) Warping: $A A E_{*}(*, *)=$ $0.836 \pm 0.361$

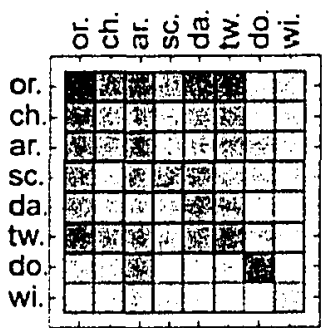

(b) CGSM:

$A . A E_{0}(*, *)=$ $0.904 \pm 0.181$

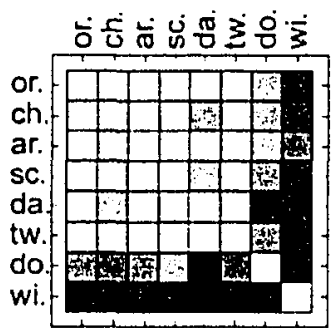

(c) Warping: $R R_{*}(*, *)=$ $0.551 \pm 0.251$

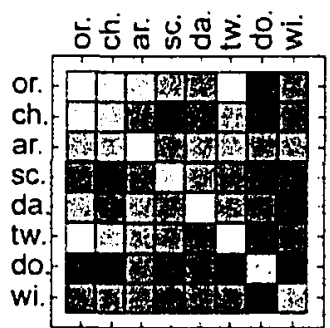

(d) CGSM: $R R_{*}(*, *)=$ $0.36 \mathrm{~T} \pm 0.168$

Figure 6.12: $A A E_{*}$ and $R R_{*}$ over all pairings of the eight office image collections. See figure 4.9 for notation. $A A E \max .=1.811, R R \max .=1$.

\subsection{Comparative Results}

As described in chapter 3 , we can compare the $A A E$ metric of two homing methods with a paired t-test. The $R R$ metric can be compared with the nonparametric test, Wilcoxon 
Significance of difference in $A A E_{*}$

\begin{tabular}{|l|l|l|l|l|}
\hline & original & hall1 & hall2 & cross \\
\hline Warping & & $* * * *$ & $* * *$ & \\
\hline CGSM & & & & \\
\hline
\end{tabular}

Significance of difference in $R R_{*}$

\begin{tabular}{|l|l|l|l|l|}
\hline & original & hall1 & hall2 & cross \\
\hline Warping & & $* *$ & $* *$ & $* * * *$ \\
\hline CGSM & & & & \\
\hline
\end{tabular}

Table 6.1: Statistical significance of the difference in $A A E_{*}$ and $R R_{*}$ between warping and CGSM. Significance for each cell is indicated if the corresponding method is significantly better (lower $A A E$ or higher $R R$ ) than the other method. Empty fields indicate no significant difference. For $A A E$ a paired t-test was used. For $R R$ the Wilcoxon matched pairs test was used. Legend: ${ }^{*}=(p<0.05),{ }^{* *}=(p<0.01),{ }^{* * *}$ $=(p<0.001),{ }^{* * * *}=(p<0.0001)$.

matched pairs. The results of these tests applied to Warping and CGSM are shown in table 6.1 .

For image collection original there is no significant difference between Warping and CGSM in either $A A E$ or $R R$. However, for both hall1 and hall2 Warping performs significantly better in both metrics. Warping also exhibits significantly higher $R R$ in the cross-collection setup. However, there is no significant difference in $A A E$ for the crosscollection.

These results generally confirm the qualitative results found above. Although both methods are on equal footing in original, Warping clearly has the advantage in all other tested environments.

\subsection{Robot Homing Trials}

To provide further justification for the results presented above, a series of online homing trials was carried out as described in chapter 3 .

Figure 6.13(a) and 6.13(b) show traces of the robot homing according to Warping and CGSM, respectively. The number of successful homing attempts for Warping was 20 out of 24. In the left two plots of figure 6.13(a) we see that Warping was able to home to the vicinity of the top two snapshot positions from all start positions. However, the right two 
plots show that homing to the bottom two snapshot positions was successful only from the bottom four start positions. This matches the offline results on the image database where Warping is found to perform most poorly for those snapshot positions near the lower end of the capture grid.

The number of successful homing attempts for CGSM was 16 out of 24 . The most difficult snapshot position for CGSM is that shown in the second plot of figure 6.13(b). That this worst position is in the upper right is consistent with the offline results. For this snapshot position only 3 of 6 start positions lead to successful homing. At start position '4', the robot approached near enough to an obstacle to register a collision on its very first move. Thus, we see no trace emanating from ' 4 '. The best performance is found for the lower right snapshot position (rightmost plot in figure 6.13(b)). Homing is successful here for 5 of 6 start positions. In fact, it is almost successful from all 6 . One trace shows the robot homing to the snapshot position, but after several oscillations the trace leads off towards the upper right.

The online trials do not by themselves provide a clear indication of which method yields the best homing performance. They do, however, lend credence to the offline results as the areas of good and bad performance seem to correlate between these two experimental methodologies. Further, these trials are invaluable in demonstrating that both methods have applicability in the real world. This is an extremely important statement as it validates both methods as viable technical approaches to robot homing.

\subsection{Analysis}

While CGSM performs on par with Warping on image collection original, it performs less well on hal11, hal12, and on the cross-collection setup. The computed values for $A A E$ are higher on the hall collections than on original and the values for $R R$ are lower. Similar to the analysis of COMALV in chapter 4 , we ask the question what aspect of original allows CGSM to succeed that is either not present or is altered in the other collections. We will examine two possible factors to explain the difference in performance between the office environment and the hall environments. These two factors are feature reliability and density.

At different positions in the environment, different features will pass in and out of visibility. Some features become obscured by other objects while others are revealed. Additionally, some detected features may not correspond to any visible object. The Harris corner detector used here will respond to two crossed edges. For example, at position a an edge from a 

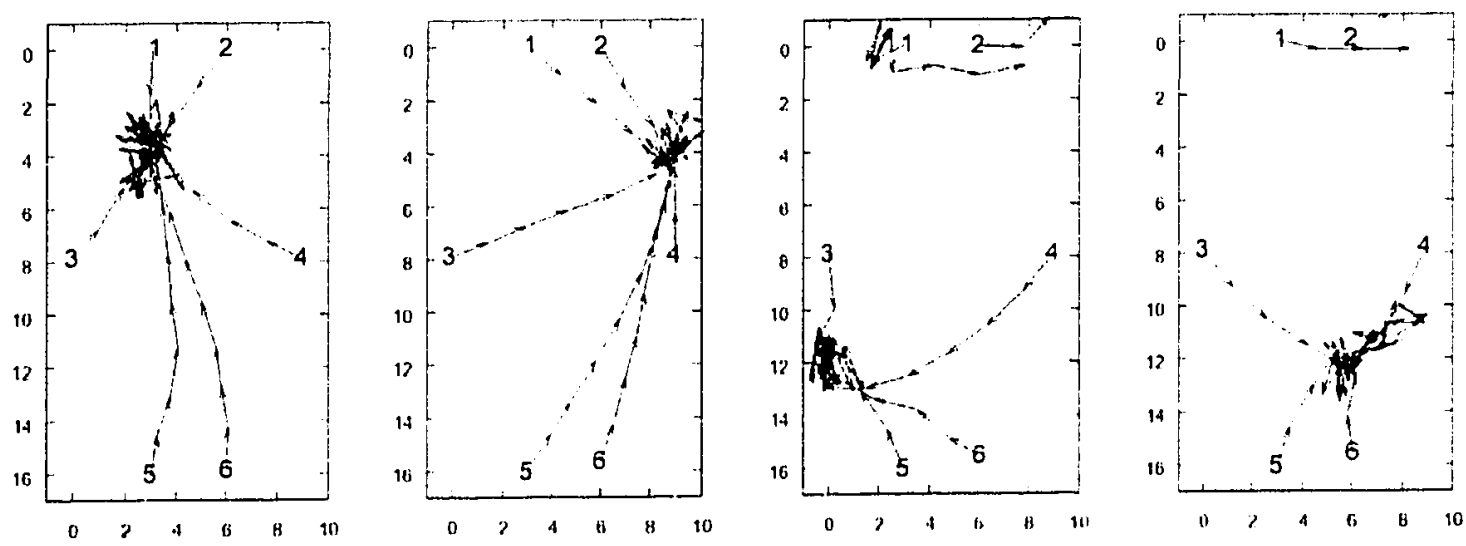

(a) Warping
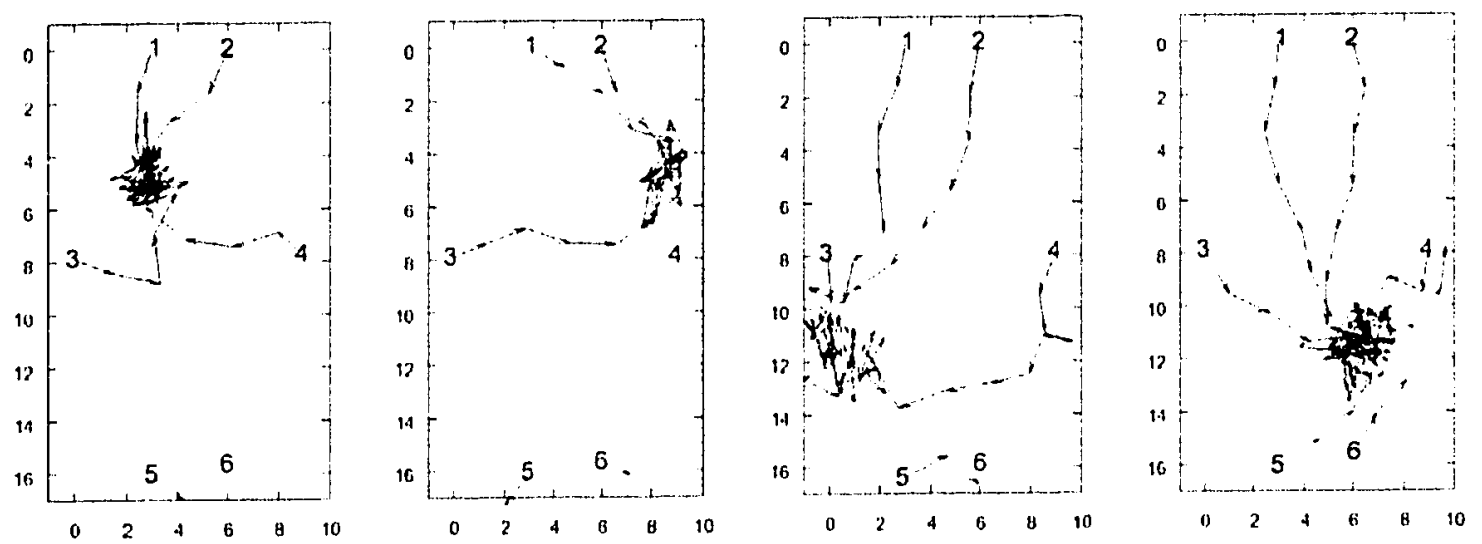

(b) CGSM

Figure 6.13: Performance of Warping and CGSM when tested live on the Pioneer robot. Numbers indicate start positions and intersecting dashed lines indicate snapshot positions. 
tabletop might intersect the edge at the seam of two perpendicular walls. At position $\mathbf{b}$ the tabletop's edge no longer intersects the other edge. The feature detected at a was particular to that position and became completely unavailable at $\mathbf{b}$. To find correspondences between features requires those features to be reliable.

One possible means to measure the reliability of features is simply to count them. Ideally; a fixed number of features should be detected at all positions in the environment. Figure 6.14 shows the number of features detected by CGSM for all images in original, hall1, and hal12. A rough measure of feature reliability across an image collection is the standard deviation of the number of features. High reliability corresponds to low standard deviation. As CGSM performs better on orignal than either hall1 or hall2 we would expect that the standard deviation of the number of features should be lowest for original. However, this is not the case. The captions in figure 6.14 show these standard deviations and the lowest is actually found in hal11. Thus, differences in feature reliability do not readily explain the performance difference of CGSM between orignal and the hall collections.

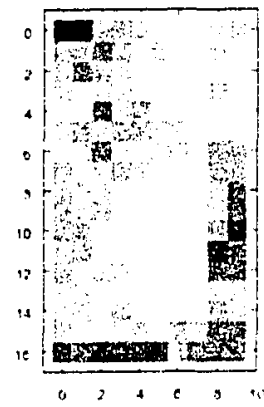

(a) original, avg. $=7.241$, stcl. $=2.681$

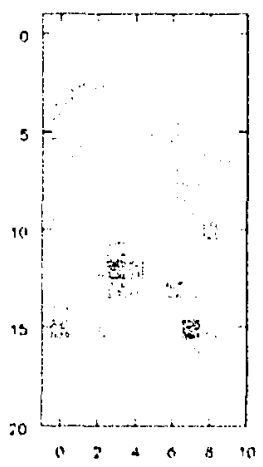

(b) hall1, avg. $=11.065$ std. $=2.61 .4$

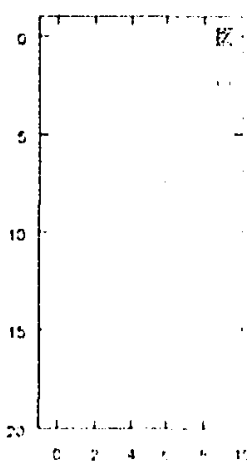

(c) hall2,

avg. $=14.225$. st.d. $=5.683$

Figure 6.14: Number of features detected by CGSM with parameters as given in section 6.6 .

It is important to note that umreliability in feature detection is still a major problem for CGSM. Large variations in the number of detected features have a direct impact on homing performance. Recall that the worst performance on the original collection occurred for the upper right portion of the capture grid. This can be correlated with large changes in the number of detected features in the same portion of the grid, as can be seen in figure 6.14 . The maximum cell-to-cell change in the number of detected features is found for position 
$(9,1)$, in the upper right of the grid.

Another possible factor to explain the difference in performance between the office and hall environments is the density of features. If we allow too few features then the impact of false correspondences becomes great. On the other hand, if too many features are allowed then there becomes more opportunity for confusing nearby features, and therefore a higher likelihood of establishing false correspondences. The density of features, of course, is the number of features per unit area. As the area of images is constant it is equivalent to describe the number of features. The number of features per image has been plotted already in figure 6.14. The captions of that figure also give the average number of features in each image collection. Notice that this average is lower for original than for hall1 or hall2.

Three of CGSM's parameters control the density of extracted features. These include the radius of neighbourhood scanned by the Harris corner detector, the number of applications of the Gaussian to produce image GaussHarris, and the threshold for selecting the strongest corners. Of these, the most direct impact on feature density comes from thresholding. Feature density decreases with increasing threshold. Figure 6.15 plots $A A E$ * over twenty start positions for a range of threshold values. As reflected in the parameter search, the minimum value for original occurs at 0.02 . This value occurs in a rather deep valley, meaning that deviations from this threshold have a large negative impact. A shallow valley for hall2 occurs at 0.0075 . Interestingly, the minimum value for hall1 occurs at zero. Unfortunately; it appears that no one threshold value is ideal for all three image collections.

Optimizing parameters for hall1 or hall 2 would clearly improve performance on those image collections. To perform this optimization, an agent would have to explore the new environment and test various parameter settings. This could be achieved by starting out from the goal position and maintaining a ground truth home vector by path integration. The vector computed by CGSM could then be compared with the ground truth vector, allowing parameters to be adjusted via some learning algorithm. However, as discussed in section 4.3.4, such a strategy incurs additional costs in time and energy. It would be preferable to develop a method less sensitive to parameter settings which could operate in many environments without customization.

\subsection{Complexity and Biological Plausibility}

The wiring diagrams presented in this chapter were intended to illustrate how various operations could be implemented in a retinotopic matrix. Although not all operations were illustrated in this manner, nearly all of CGSM's operations are retinotopic-compatible. 


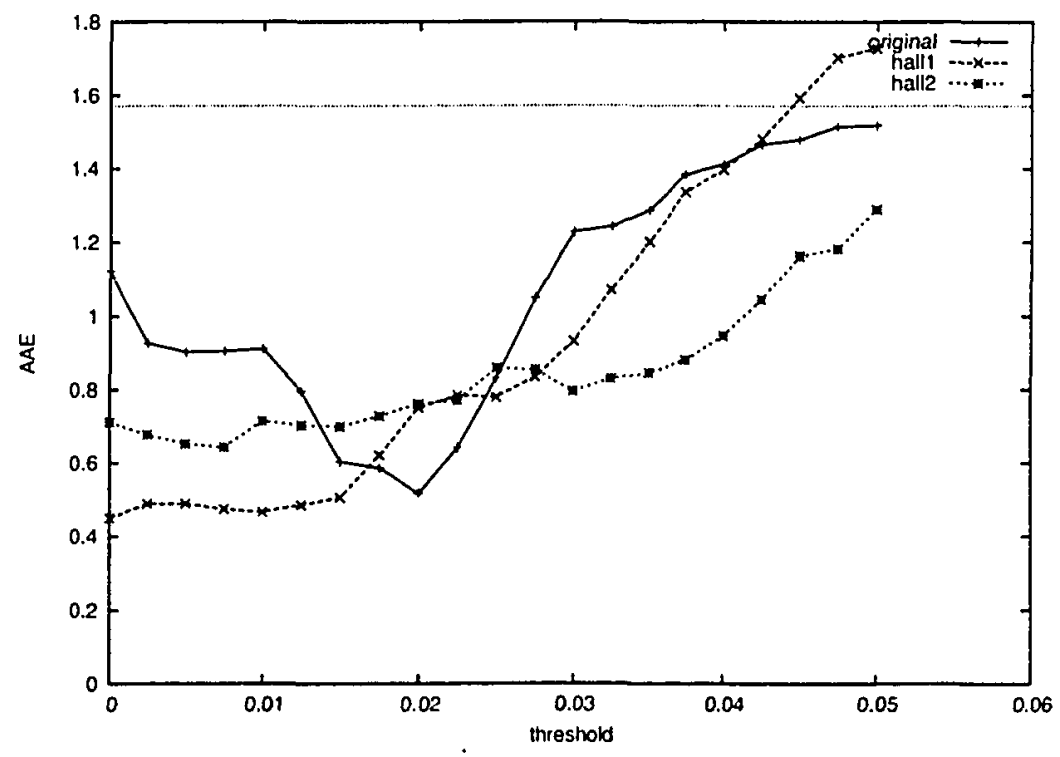

Figure 6.15: Plot of $A A E_{*}$ versus threshold value for image collections original, hall1, and hall2.

The exceptions are the operation to grow the potential surface, and the summation of home vectors. The potential surface operation does exhibit retinotopic connectivity but needs to be applied in an iterative fashion. Fortunately, this expensive operation can be offloaded to the snapshot image processing matrix. The summation of per-correspondence home vectors into a single home vector is a convergent summing operation. Note that the repeated application of the Gaussian filter might be considered non-retinotopic. However, this repeated filter can easily be replaced by a single larger filter ${ }^{6}$. The repeated Gaussian is employed here only because it is a convenient building block for developing filters of various size.

\footnotetext{
${ }^{6}$ This fact is due to the associativity of convolution (see chapter 3 of [83] or any other introductory text on signals and systems).
} 
CGSM's snapshot processing matrix is not strictly retinotopic-compatible due to the iteration of the potential surface operation. This iteration is not necessarily biologically implausible, but it does fail to meet the stated criterion for retinotopic compatibility: On the other hand, the current processing matrix does fit all constraints for retinotopic processing. The average processing time per image on a Pentium $42 \mathrm{Ghz} P C$ was $228 \mathrm{~ms}$.

\subsection{Conclusions}

CGSM is an interesting method because it is able to correspond image features without search. It is based upon Möller et al.'s neural snapshot model but extends it in a number of ways. The most important of these extensions is its applicability to real-world images. The results presented above show that CGSM can be competitive to Warping for certain enviromments. In others, it performs less well. Yet it is apparent that it generates approximately correct home vector fields for at least the area around the goal. The online results verify CGSM's applicability to robot visual homing in a real enviromment. As discussed above, CGSM employs retinotopic-compatible for nearly all operations and the processing matrix for the current image does satisfy retinotopic processing.

Meanwhile, this method is limited in many ways. Fundamentally; it relies upon the assumption that the appropriate features to correspond between images are those which are closest. Given perfect feature detection this guarantees correct homing, but only for a small region around the goal. Of course, the method of feature detection is far from perfect. Variation in feature reliability and density were found to impact performance. The structure of the environment appears to determine the appropriate threshold value to use. This reduces the method's applicability to other enviromments.

The issue of thresholding is crucial because CGSM deals with discrete features. Corners wink in and out of existence depending upon their strength with respect to the threshold. Also, while we can describe a particular configuration of corners as being more or less distinctive, the corners themselves are completely indistinguishable. These facts compel the investigation of other approaches which escape the problems of discrete and indistinct features. In the next chapter a much richer means of describing local image patches is developed. 


\section{Chapter 7}

\section{Scale Invariant Image Descriptors}

\subsection{Introduction}

This chapter presents a novel image descriptor which can characterize local patches of an image in a scale invariant manner. The term image descriptor is similar to the term feature but suggests a richer characterization bevond the level of such depictions as "there is a corner at position $(x, y)$ " or "there is an edge of orientation $\theta$ at position $(x, y)$ ". An image descriptor extracts some information from a small region of an image, referred to as an image patch. A scale invariant image descriptor extracts such information that will not significantly change, even if the image patch is subject to scaling. Scaling is a prevalent source of image distortion in visual homing where currently viewed landmarks generally appear larger or smaller than in the snapshot image. Another important facet of this image descriptor is its simple repetitive structure. A wiring diagram similar to those in the previous chapter will show how this descriptor can be implemented in a retinotopic matrix.

A number of scale invariant image descriptors have been proposed in the literature. A review and comparison between the major approaches is provided by Mikolajczyk and Schmid [69]. Miliolajczyk and Schmid find that approaches based on Lowe's [65, 66] SIFT descriptor perform the best. Another scale invariant descriptor was proposed by Rizzi et al. [88] based on the Fourier-Mellin transform. This descriptor is of interest because it was developed specifically for visual homing. However, it is currently unclear how complex these schemes might be for implementation in the neural hardware of an insect.

In addition to developing a new local image descriptor, this chapter presents two methods for visual homing based on this descriptor. The first method searches for correspondences between descriptors in the snapshot image and those in the current image. However, 
the second method takes advantage of the structure of the motion field for pure translation to avoid this search process. This method only pairs descriptors at the same image position. Very similar pairs ideally correspond to one of the two stationary points in the motion field. These points are the focus of contraction (FOC) and the focus of expansion (FOE), introduced in chapter 2. Finding either of these foci is equivalent to solving the visual homing problem. Some of the content of this chapter has been previously published $[101,102]$.

\subsection{Scaling Model}

We begin by defining a model of image scaling applicable to a local image patch. Let $\mathbf{p}$ be the coordinates of the centre of an image patch. The effect of scaling is to change the distance of image features to $\mathbf{p}$ by a factor $k$. Features nearby to $\mathbf{p}$ will shift by a smaller amount then distant features, yet the same scaling factor of $k$ is applied for all image features. Hence, we refer to this as linear scaling.

Assume we have an image $I$ which has been subject to linear scaling about point $\mathbf{p}$ by factor $k$. A point $\mathbf{a}$ in the original image $I$ now corresponds to a point $\mathbf{a}^{\prime}$ in the scaled image $I^{\prime}$. That is, a pixel in the original image at a will have the same value as a pixel in the scaled image at $\mathbf{a}^{\prime}$. Therefore

$$
I(\mathbf{a})=I^{\prime}\left(\mathbf{a}^{\prime}\right)
$$

Note that $I(\mathbf{a})$ is shorthand for the value of the pixel in image $I$ with coordinates $\left(a_{x}, a_{y}\right)$. Also, for simplicity we ignore pixel discretization and treat a as real-valued.

We now formulate an expression for a which involves the centre of scaling $p$. The following parametric equation of a line represents a with respect to its distance $l$ from $p$, and with respect to the direction from $p$ to a indicated by the unit vector $v$.

$$
\begin{array}{r}
\mathbf{a}=\mathbf{p}+l \mathbf{v} \\
\mathbf{v}=\frac{\mathbf{a}-\mathbf{p}}{\|\mathbf{a}-\mathbf{p}\|}
\end{array}
$$

The point $\mathbf{a}^{\prime}$ corresponding to a after linear scaling is similarly represented.

$$
\mathbf{a}^{\prime}=\mathbf{p}+k \cdot \mathbf{v}
$$

Note that this scaling model assumes the scaling factor $k$ to be constant across the whole image. This is generally not true for the panoramic images employed here. However, linear scaling is a reasonable model for the scaling that occurs within local image patches of a panoramic image. 


\subsection{The Scale Invariant Descriptor}

In this section we develop a local image descriptor which is partially invariant to scale changes. Figure 7.1 shows an image $I$ and two increasingly scaled variants $I^{\prime}$ and $I^{\prime \prime}$. The figure also plots the value of each image along the ray $\mathbf{p}+l \mathbf{v}$ where $l>0$ and $\mathbf{v}$ is arbitrarily set on a diagonal. We refer to this ray as a channel. The house image consist only of edges so the plots show isolated pulses where the chamnel crosses an edge.
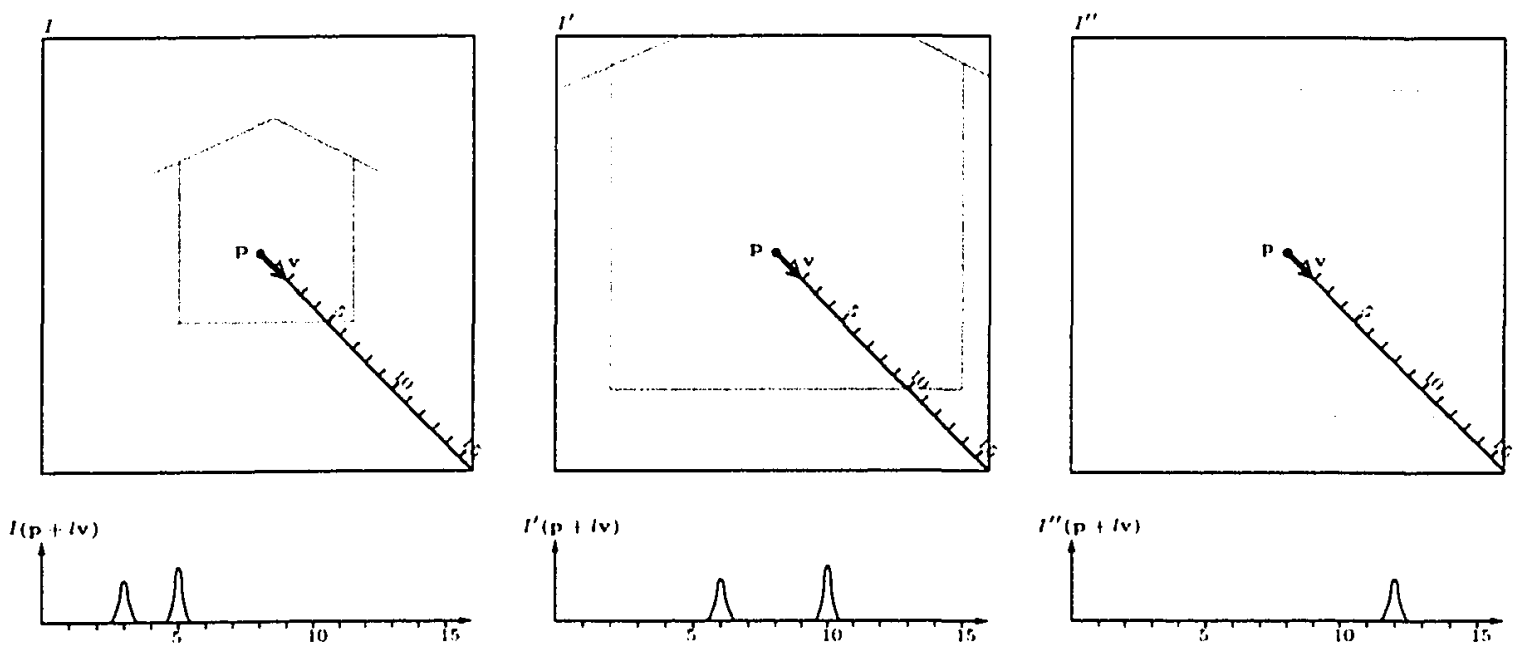

Figure 7.1: Scaling of an image $I$ and the value of $I$ along a channel. Three images are shown with scale doubling incrementally from left to right. Beneath each image is a plot of the image values along the indicated chammel. The images consist only of edges with darker edges having a higher value than lighter edges.

It can be observed that while the positions of edge pulses along the channel have changed between $I$ and $I^{\prime}$, the same two pulses are still found. Hence, the area underneath these two pulses is the same. This observation prompts our first proposal for an invariant measure, which is the sum of image values along the channel

$$
f^{\mathbf{p}, \mathbf{v}, I}=\int_{0}^{l_{\text {max }}} I(\mathbf{p}+l \mathbf{v}) d l
$$

If indeed the same pulses are found along the same channel of $I$ and $I^{\prime}$ then the following is true

$$
f^{\mathbf{p}, \mathbf{v}, I^{\prime}}=f^{\mathbf{p}, \mathbf{v}, I}
$$


However, if the scaling factor $k$ is too large then this condition will not hold. For example, in image $I^{\prime \prime}$ of figure 7.1 the outside edge of the house has been scaled entirely out of the frame. The channel now shows only a single pulse. Thus, $f^{\mathbf{p}, \mathbf{v}, I} \neq f^{\mathbf{p}, \mathbf{v}, I^{\prime \prime}}$. The same problem occurs for contraction $(k<1)$. If $I^{\prime \prime}$ was the original image and had been scaled down to $I$, the pulse representing the outside of the house would have appeared-and again $f^{\mathbf{p}, \mathbf{v}, I} \neq f^{\mathbf{p}, \mathbf{v}, J^{\prime \prime}}$. To mitigate the problem of appearance/disappearance of image features we propose a new invariant measure which includes a decay function

$$
g^{\mathbf{p}, \mathbf{v}, I}=\int_{0}^{l_{\max }} w(l) I(\mathbf{p}+l \mathbf{v}) d l
$$

The purpose of the decay function $w()$ is to reduce the impact of outlying features on g. Appendix A includes a derivation which places some constraints on $w()$. One obvious function which satisfies these constraints is

$$
w(l)=\frac{1}{l s}
$$

where $\zeta<1$.

The objective now is to determine the relationship between $g^{\mathrm{p}, \mathrm{v}, I}$ and $g^{\mathrm{p}, \mathrm{v}, I^{\prime}}$. This relationship is explored in appendix $A$ and found to be as follows:

$$
g^{\mathbf{p}, \mathbf{v}, I^{\prime}} \approx k w(k) g^{\mathbf{p}, \mathbf{v}, I}
$$

The presence of the factor $k w(k)$ implies that $g$ is not scale invariant. We will cleal with this problem momentarily: More fundamentally, however, is the fact that a scalar quantity such as $g^{\mathrm{p}, \mathrm{v}, I}$ is likely insufficient to describe a local image patch robustly: A richer clescriptor is required to allow image patches to be disambiguated. We obtain such a descriptor by forming a vector $\mathbf{g}$ of $g$ values computed from the same point $\mathbf{p}$ but at different directions

$$
\mathbf{g}^{\mathbf{p}, I}=\left(\begin{array}{c}
g^{\mathbf{p}, \mathrm{v}_{0}, I} \\
g^{\mathbf{p}, \mathrm{v}_{1}, I} \\
\vdots \\
g^{\mathbf{p}, \mathrm{v}_{m}, I}
\end{array}\right)
$$

The length of the vector $\mathbf{g}$ is $n$. An obvious choice for the channel direction vectors $\mathbf{v}_{i}$ is to arrange them evenly in a radial pattern. For example, if $n=4$ we would choose left, up, right, and down. If $n=8$ we would add the four diagonals as well.

For the first algorithm presented below we will not be concerned with the length of $\mathbf{g}$, but only its direction. Therefore we define a normalized vector $\mathbf{h}$

$$
\mathbf{h}^{\mathrm{p}, I}=\frac{\mathrm{g}^{\mathrm{p}, I}}{\left\|\mathrm{~g}^{\mathrm{p}, I}\right\|}
$$


By normalizing we remove the factor $k w(k)$, hence

$$
\mathrm{h}^{\mathrm{p}, I^{\prime}} \approx \mathbf{h}^{\mathrm{p}, I}
$$

and we can say that $\mathbf{h}$ is a scale invariant image descriptor.

For the second algorithm it will be necessary to know whether $k$ is greater or less than one. Thus, in the description for this algorithm we will also make reference to $\mathrm{g}$.

\subsubsection{Conditions}

The image descriptor $h$ is invariant to scale changes given the following qualitative conditions:

1. The scale factor $k$ is neither too great nor too small. The decay function can offset the impact of edge pulses being scaled in and out of range, but the scaling of outlying edge pulses will still generally distort the direction of $\mathbf{h}$.

2. If image edges are particularly dense then the edge pulses along a channel may interfere with each other in the summation of equation $(\bar{T} . \bar{T})$. Thus, it is advantageous for image edges to be relatively sparse.

\subsubsection{Structure}

The computation of $\mathbf{g}$ involves only the weighted summation of input image values. Figure 7.2 depicts how all necessary computations can be performed within a retinotopic matrix.

\subsection{SIMatch}

We present here the first of two homing methods which use the image descriptor developed above. We will refer to this method as SIMatch, which abbreviates Scalc Invariant Matching. This method is based on matching each descriptor in the snapshot image to $N$ descriptors in the current image at neighbouring inage positions. The coordinates of the best matches are then used to generate correspondence vectors. These correspondence vectors are then mapped to home vectors using the method described in chapter 5 . The resulting home vectors are then summed and normalized to yield a single home vector.

It must be commented upon SIMatch violates the constraints outlined in the introductory chapter. Matching descriptors between images requires a search process. Therefore 


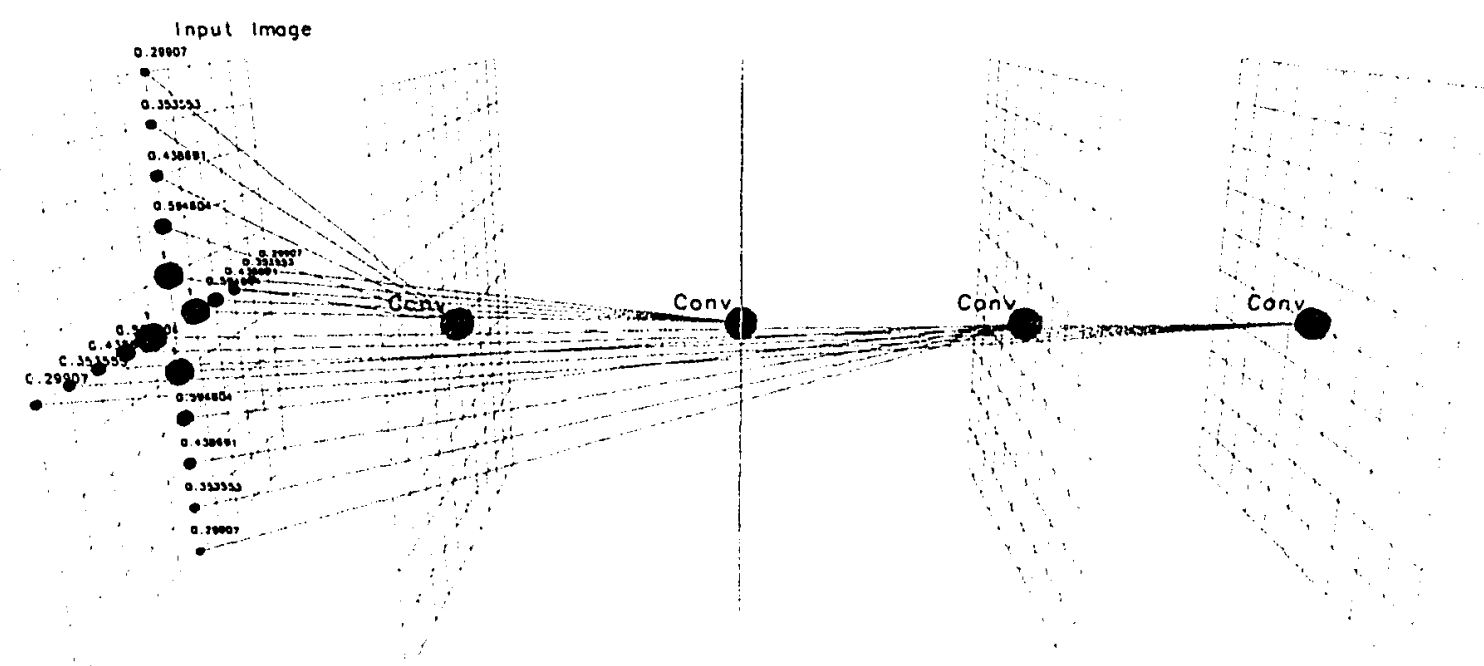

Figure 7.2: Retinotopic matrix to compute g. This descriptor uses the paraneters $n=4, l_{\max }=5$, and $\zeta=0.75$. See figure 6.2 for notation.

the appearance of this method may strike the reader as surprising. However, this method plays an important part in the development of ideas subsequently presented in this thesis.

We refor to the positions of descriptors in the snapshot image $S$ as origin points. Each origin point is matched with descriptors in the current image $C$ at $N$ candidate points. These candidate points are located in a block surrounding the origin point.

For each origin point $\mathbf{p}$ in $S$ we search to find the candidate point $\mathbf{p}^{\prime}$ in $C$ which is at the centre of the image patch most similar to the image patch at $p$. To judge the degree of match between these two image patches we compute the scale invariant descriptors $\mathbf{h}^{\mathbf{p} . S}$

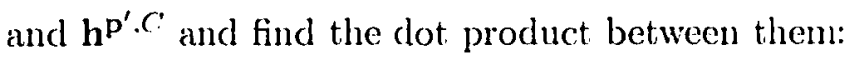

$$
\mathrm{DP}\left(\mathbf{p}, \mathbf{p}^{\prime}\right)=\mathbf{h}^{\mathbf{p} . S} \cdot \mathbf{h}^{\mathbf{p}^{\prime}, C}
$$

A high value of DP indicates a good match.

To reduce computational complexity we do not consider all positions in $S$ as origin points, but only a sampling of positions at integer multiples of the horizontal step size $m_{x}$ and the vertical step size $m_{y}$, where $m_{x}$ and $m_{y}$ are also integers. Given images of width $w$ pixels and height $h$ pixels we define the number of horizontal and vertical sampling points

$$
\begin{aligned}
& n_{x}=\left\lfloor w / m_{x}\right\rfloor \\
& n_{y}=\left\lfloor h / m_{y}\right\rfloor
\end{aligned}
$$


The total number of origin points is $n_{x} n_{y}$. Each origin point requires a search for the best candidate point. This search involves computing DP for $N$ candidate points. The candidate points are located within a radius of $q$ pixels from the origin point $p$. Hence, $N=(2 q+1)^{2}$.

We select $\check{p}$ as the candidate point with the highest DP:

$$
\begin{gathered}
\check{\mathbf{p}}=\arg \max _{\mathbf{p}^{\prime} \in E_{q}(\mathbf{p})} \operatorname{DP}\left(\mathbf{p}, \mathbf{p}^{\prime}\right) \\
E_{q}\left(\left(p_{x}, p_{y}\right)\right)=\left\{\left(p_{x}+i, p_{y}+j\right)|i, j \in \mathbb{Z},| i|\leq q \wedge| j \mid \leq q\right\}
\end{gathered}
$$

There is an additional constraint made on the correspondence search whereby origin points in the snapshot image will only be paired with candidate points which are on the same side of the horizon. As described in section 6.5.5, as long as the robot moves purely within a single plane, no image features should cross the horizon. Therefore we constrain our search to avoid any such spurious matches.

The candidate point $\check{\mathbf{p}}$ with the highest DP is used to compute the correspondence vector $\delta$

$$
\delta=\left(\begin{array}{l}
\delta_{x} \\
\delta_{y}
\end{array}\right)=\left(\begin{array}{c}
\Delta_{x}\left(\check{p}_{x}-p_{x}\right) \\
\Delta_{y}\left(\check{p}_{y}-p_{y}\right)
\end{array}\right)
$$

where $\Delta_{x}$ represents the inter-pixel angle in the horizontal direction and $\Delta_{y}$ represents vertical inter-pixel angle.

Home vectors are computed from the correspondence vectors using the vector mapping technique of chapter 5 . The final home vector is obtained by summing the individual home vectors and normalizing the result.

\subsection{SIPair}

We now present our second homing method based on the scale invariant image descriptor. This method is called SIPair, which abbreviates Scalc Invariant Pairing. While SIMatch repeatedly searches for correspondences between origin points in $S$ and candidate points in $C$, SIPair considers only one candidate point for each origin point. Only points at the same image position are compared and the best matching pair is used to compute a final home vector directly. Unlike the previous method, this method operates in accordance with retinotopic processing.

In general, a landmark seen in the snapshot image will either move to a new position in the current image, or will disappear. However, there is an important exception to this rule. Landmarks at the FOC or FOE will maintain the same image position if the displacement of the agent from the goal consists of pure translation. Chapter 2 discussed this topic 
extensively. It is assumed here that the agent's rotation has been restored to the rotation held at the snapshot. Therefore, both foci should exist and be detectable within a panoramic image. As the foci are points of zero flow the image patches surrounding them can be compared between the snapshot and current images without involing a search process.

SIPair computes descriptors $\mathbf{g}$ and $\mathbf{h}$ for positions along the horizon of the snapshot image $S$ and the current image $C$. Descriptors at the same image position in both images are then compared by computing the dot product between them. The coordinates $\hat{\mathbf{p}}$ of the pairing with the highest DP value is determined

$$
\hat{\mathbf{p}}=\arg \max _{\mathbf{p}} \mathrm{DP}(\mathbf{p}, \mathbf{p})
$$

If our descriptor truly provides a unique means of characterizing local image patches then $\hat{\mathbf{p}}$ represents a local image patch that is stationary between the snapshot image and current image. Such an image patch could either represent a very distant landmark, or else it could represent one of the foci. Here we assume the latter. The experiments below will show whether this assumption is sound or not.

We determine which of the two foci corresponds with $\hat{\mathbf{p}}$ by comparing the length of the vector $\mathbf{g}^{\dot{\mathbf{p}}, S}$ with $\mathbf{g}^{\dot{\mathbf{p}}, C}$. Growth of the descriptor vector $\mathbf{g}$ from the snapshot to the current image occurs in the neighbourhood of the FOC. By definition, image features move in towards the FOC, and as they do, become weighted more heavily by the decay function $w()$. The opposite situation occurs at the FOE where features become weighted less heavily as they expand away from the FOE. The quantity $b$ equals 1 for the case of contraction and -1 for expansion

$$
b=\left\{\begin{aligned}
1 & \text { if }\left\|\mathbf{g}^{\mathbf{p}, S}\right\|<\left\|\mathbf{g}^{\mathbf{p}, C}\right\| \\
-1 & \text { if }\left\|\mathbf{g}^{\mathbf{p}, S}\right\|>\left\|\mathbf{g}^{\mathbf{p}, C}\right\| \\
0 & \text { otherwise }
\end{aligned}\right.
$$

Finally, the computed home vector is given by converting the image coordinate $\mathrm{p}$ into a vector and using $b$ to reverse that vector if appropriate

$$
\mathbf{w}=b\left(\begin{array}{c}
\cos \left(\Delta_{x} p_{x}\right) \\
\sin \left(\Delta_{y} p_{y}\right)
\end{array}\right)
$$

The vector $\mathrm{w}$ above is the final estimated home vector.

\subsubsection{Pre-Processing}

Both homing methods require edges to be extracted from input images. A Sobel filter is applied for this purpose. Prior to applying the Sobel filter, the input images are smoothed by 
a Gaussian filter. The parameter search described below varies the number of applications of this pre-Sobel filter. As described in section 7.3.1, it is not advantageous for image edges to be excessively dense. The density of edges can be reduced by passing the image through a power filter, which raises each pixel's value to exponent $\tau$.

\subsection{Optimizing Parameters}

Some parameters of these two homing methods are method-specific while others are shared by both methods. The method-specific parameters were set to values which generally appeared to provide good results. For SIMatch these included the search radius $q$ (set to 30 ), the horizontal step size $m_{x}(4)$, and the vertical step size $m_{y}(4)$. Another parameter excluded points in the specified number of image rows at the top and bottom of the image from being used as origin points. This parameter (set to 10) was introduced upon observing that image patches in the top and bottom portions of the image tended to be relatively indistinct. For SIPair the only method-specific parameter is the height of the window around the horizon (9).

For the remaining shared parameters a search was carried out to find the best settings for each method. The parameters tested are as follows:

- Length of clescriptor vectors, $n: 8,32$, or $\underline{64}$.

- Exponent of decay function, $\zeta: 0.25,0.5$, or $\underline{0.75}$.

- The length of channels to sum over, $l_{\max }: 20$ or $\underline{\mathbf{5 0}}$.

- Number of applications of pre-Sobel Gaussian filter: 0 or $\underline{4}$.

- Parameter $\tau$ for post-Sobel power filter: 1,2 , or $\underline{4}$.

The best found shared parameters for SIMatch are indicated above in bold face. The best found shared parameters for SIPair are underlined.

\subsection{Results On Image Database}

\subsubsection{Sample Flow Fields}

Figure 7.3 shows some sample flow fields computed by SIMatch for snapshot position $(6,4)$ and for current image positions $(5,4)$ to $(0,4)$. These positions all lie to the left of this 
snapshot position. The image position in the current view which corresponds to the correct home direction is $(52,23)$. Thus, at this position we expect to find the focus of contraction (FOC) of the flow field. Image features will have been drawn in towards the FOC by the displacement of the agent. The FOE should be found $180^{\circ}$ opposite to the FOC. This position is at $3 * w / 4=3 * 206 / 4 \approx 155$. Indeed, contraction can be observed around the FOC and expansion around the FOE. However, there are many clearly incorrect vectors, particularly around the FOC. The overall pattern of correspondence vectors is correct, although the number of erroneous vectors increases as the displacement from the goal increases.

\subsubsection{Dot Product Images}

It is interesting to examine some of the internal workings of SIPair as well. For this method we look at the variation in $D P(\mathbf{p}, \mathbf{p})$. This quantity should show peaks at the FOC and FOE. Figure 7.4 shows $D P(\mathbf{p}, \mathbf{p})$ for snapshot position $(6,4)$ and current image positions $(6,5)$ to $(6,10)$. These positions lie below the suapshot position so the correct home vector should be directed upwards. The true location of the FOC is at $(0,23)$ while that of the FOE is $(103,23)$. Generally these images show the expected qualitative behaviour-two major peaks near the ideal locations of the FOC and FOE are found. Figure 7.4 also shows the maximum value of the dot product images which happen to all lie near the FOE.

\section{Image Collection original}

Figure 7.5 shows the home vectors generated by Warping, SIMatch, and SIPair on image collection original for snapshot positions $(6,4)$ and $(0,16)$. For position $(6,4)$ SIMatch, like Warping, exhibits a nearly perfect home vector field. For position $(0,16)$ it exhibits a vector field that is an improvement over Warping's. This improvement is shown in the lower $A A E$ and higher $R R$ found for SIMatch over Warping. The home vector field for SIPair at position $(6,4)$ is largely correct, but does show a region of incorrect vectors to the left of s. At position $(0,16)$ the performance of SIPair is much worse. For most of the left portion of the capture grid the vectors are either correct or $180^{\circ}$ incorrect. These opposite home vectors occur due to confusion about the identity of $\hat{\mathrm{p}}$. That is, whether this point represents the FOC or the FOE.

Figure 7.6 shows the computed $A . A E$ and $R R$ for the same three methods over twenty snapshot positions. SIMatch exhibits both low $A A E$ and high $R R$ throughout, except for a small region in the bottom right corner of the capture gricl. By contrast, Warping exhibits good performance except for three of the capture grid's corner regions. SIPair yields good performance only in the middle region of the capture grid. 
Pos. $(4,4)$

Pos. $(3,4)$

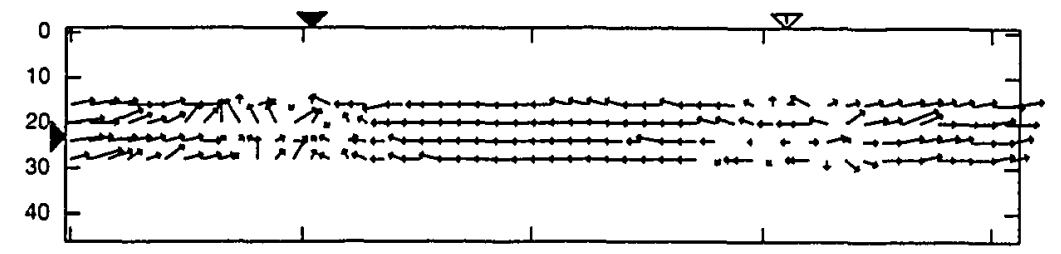

Pos. $(2,4)$

Pos. $(1,4)$

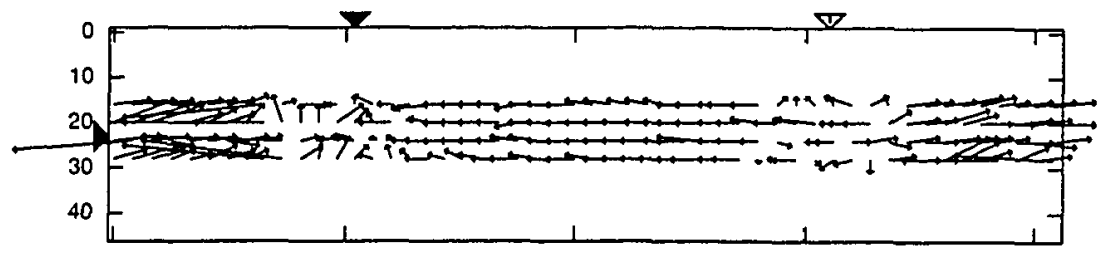

Pos. $(0,4)$
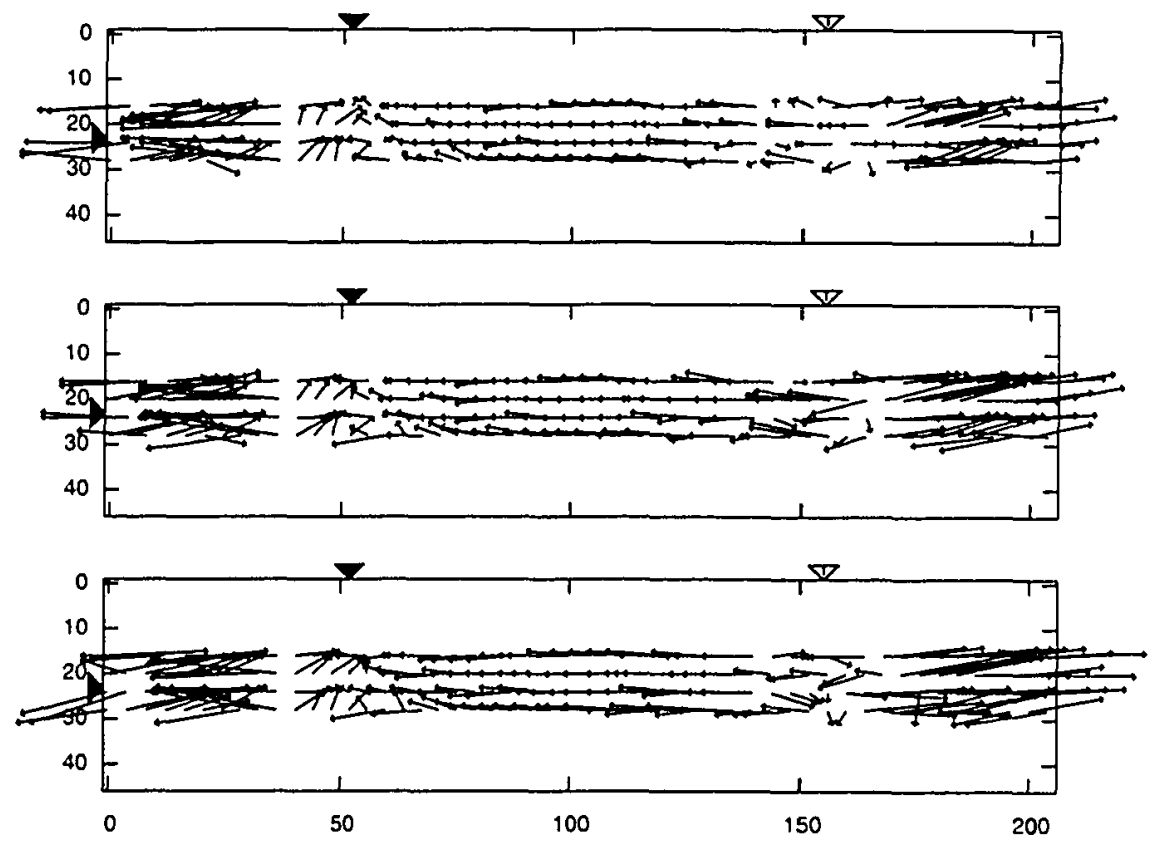

Figure 7.3: Example flow field for snapshot position $(6,4)$ and current positions from $(5,4)$ to $(0,4)$. The correct home vector is directed towards the right, meaning that the correct FOC should be around $(52,23)$ while the correct FOE should be near $(155,23)$. These positions are indicated by triangles along the plot boundary: The horizontal position of the FOC is indicated by the filled triangle, while the FOE is indicated by the open triangle. 
Pos. $(6,5)$

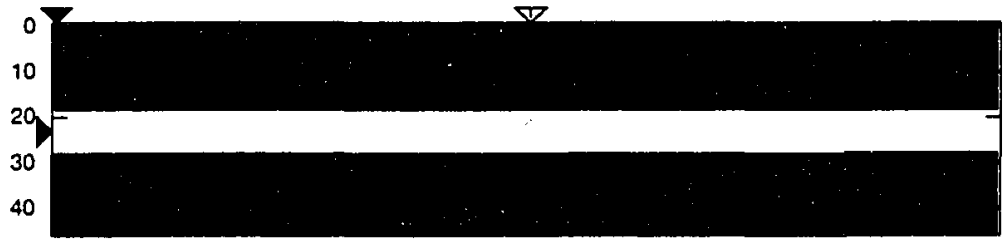

Pos. $(6,6)$

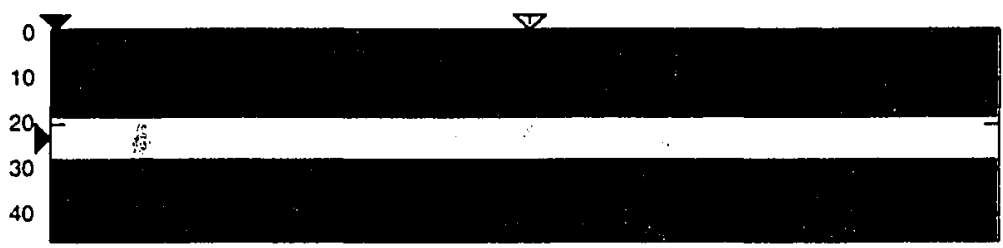

Pos. $(6,7)$

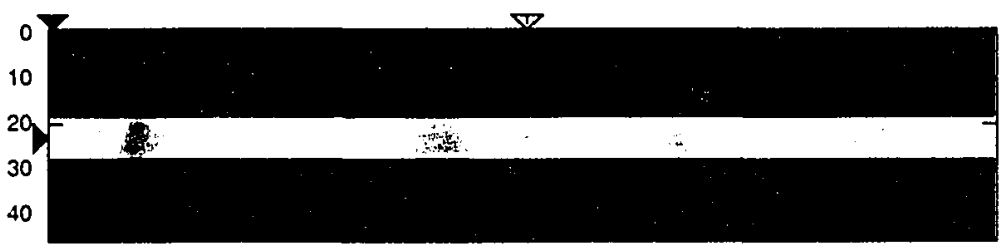

Pos. $(6,8)$

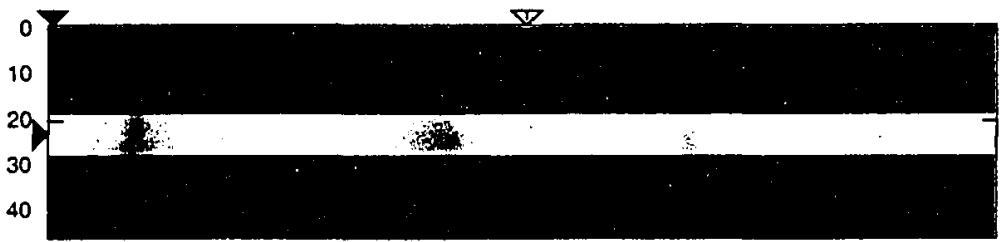

Pos. $(6,9)$
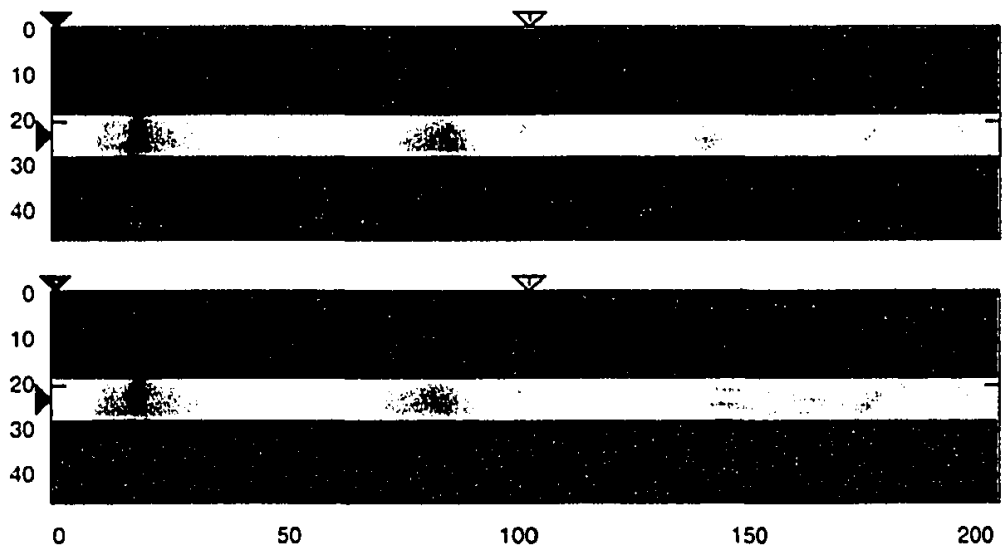

Figure 7.4: Variation in $D P(\mathbf{p}, \mathbf{p})$ for SIPair. The snapshot image was captured at $(6,4)$, which is above the positions indicated above. Hence, the correct FOC should be around $(0,23)$ while the correct FOE should be near $(103,23)$. Positions of the FOC and FOE are indicated as in figure 7.3 . For each image the location of maximum $D P(\mathbf{p}, \mathbf{p})$ is indicated by an ' $\mathrm{X}$ '. 


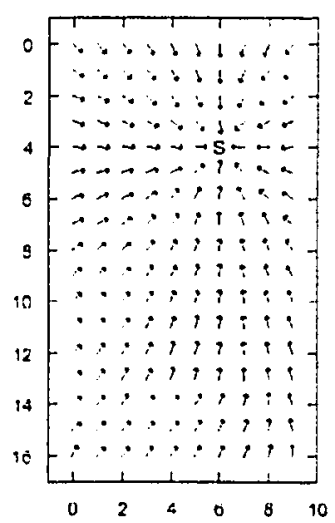

(a) Warping, $\mathbf{s}=(6,4)$ : $A A E=0.119$, $R R=1.000$

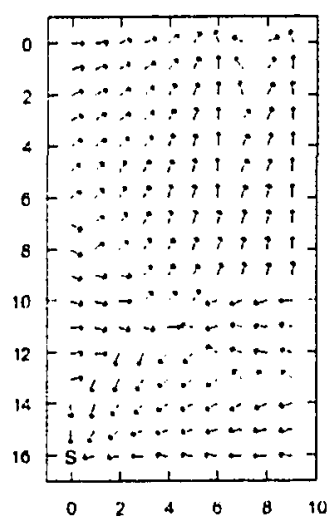

(b) Warping, $\mathbf{s}=(0,16):$ $A A E=1.758^{\circ}$, $R R=0.266$

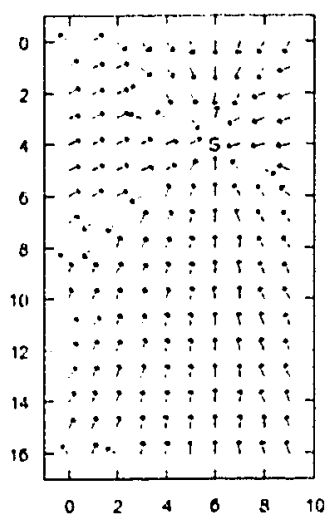

(e) SIPair, $\mathrm{s}=(6,4)$ : $A A E=0.368$, $R R=0.686$

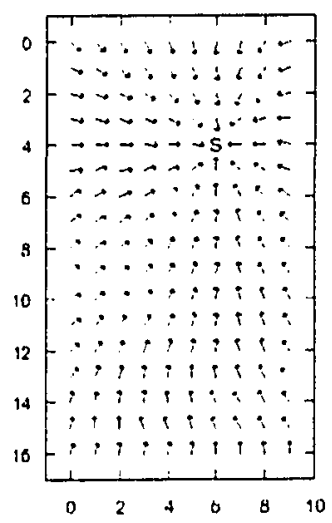

(c) SIMatch, $\mathbf{s}=(6,4)$ : $A A E=0.138$, $R R=1.000$

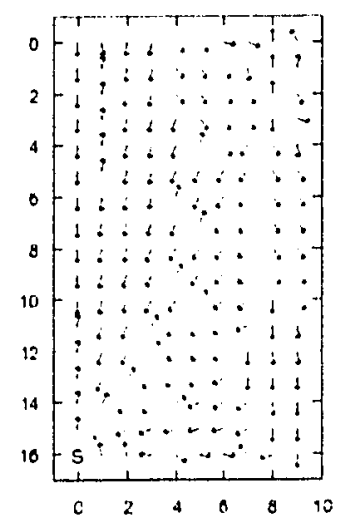

(f) SIPair, $\mathbf{s}=(0,16):$ $A A E=0.888$, $R R=0.130$

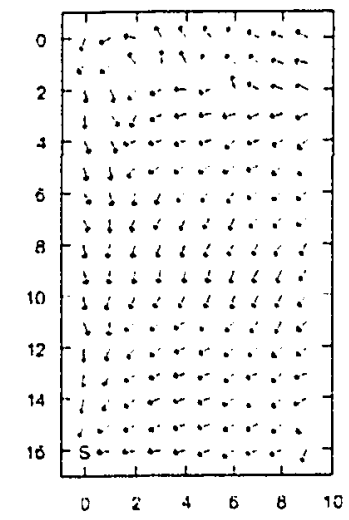

(d) SIMatch, $\mathbf{s}=(0,16):$ $A . A E=0.532$, $R R=0.858$

Figure 7.5: Home vector fields for Warping (a,b), SIMatch (c,d), and SIPair (e,f), for snapshot positions $(6,4)(a, c, e)$ and $(0,16)(b, d, f)$ in image collection original. 


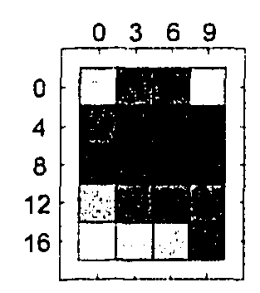

(a) Warping: $A A E_{*}=$ $0.576 \pm 0.461$

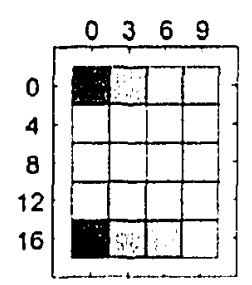

(d) Warping:

$$
R R_{*}=
$$

$0.792 \pm 0.226$

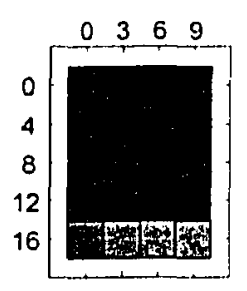

(b) SIMatch:

$A . A E_{*}=$ $0.272 \pm 0.213$

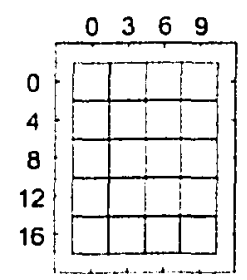

(e) SIMatch:

$$
R R_{*}=
$$

$0.962 \pm 0.0806$

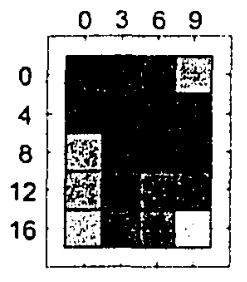

(c) SIPair: $A A E_{*}=$ $0.517 \pm 0.249$

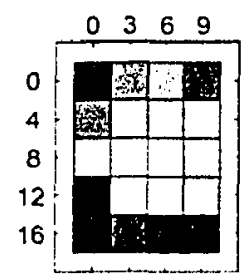

(f) SIPair:

$$
R R_{*}=
$$

$0.505 \pm 0.306$

Figure 7.6: $A A E$ and $R R$ across snapshot positions for all methods on image collection original. Beneath each plot is shown the mean value of the metric ( $A A E$, or $R R_{*}$ ) and its standard deviation (the number following the ' \pm ' symbol). Average values for $A A E$ max. $=1.758, R R$ max. $=1$.

\section{Image Collections hall1 and hall2}

Figure 7.7 shows home vectors generated by Warping, SIMatch, and SIPair on the hall image collections at snapshot position $(3,12)$. Again we see that the home vectors generated by SIMatch are about as good or better than those of Warping. In particular, SIMatch performs better in hall2. On the other hand, SIPair's performance is quite poor. There are a very small number of correct vectors embedded in the vector fields for SIPair, as well as a small number of $180^{\circ}$ incorrect vectors. However, this method is clearly failing in the hall environments.

Figures 7.8 and 7.9 shows the computed $A . A E$ and $R R$ across twenty snapshot positions for each of the hall collections. Qualitatively, the performance of SIMatch and Warping are similar in both collections. They each show small regions where performance is poor and large regions where performance is good. As expected from the home vector fields above, SIPair's performance is poor throughout. 


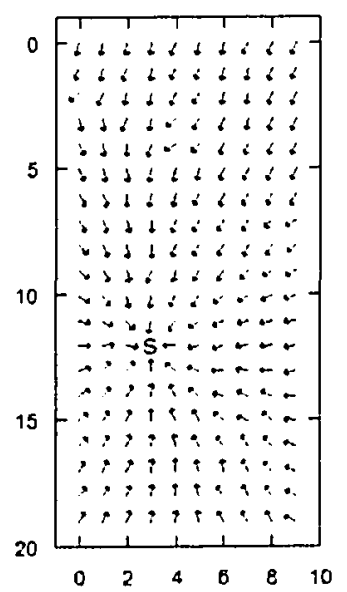

(a) Warping, hall1, $\mathrm{s}=(3,12)$ : $A A E=0.160$, $R R=1.000$

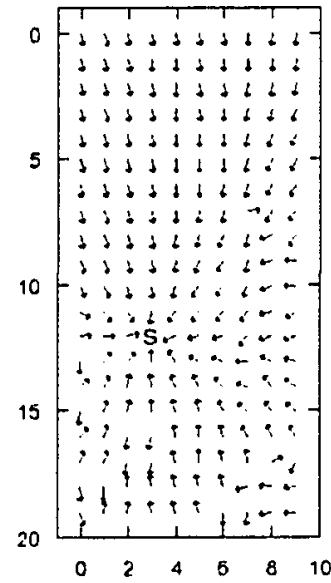

(b) Warping, hall2, $\mathrm{s}=(3,12)$ : $A .4 E=0.40 \mathrm{~T}$, $R R=0.915$

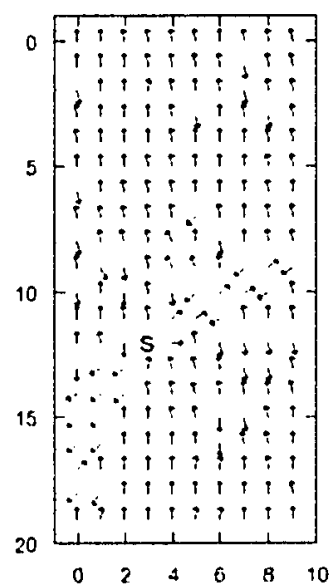

(e) SIPair, hall1, $\mathrm{s}=(3,12)$ : $A .4 E=1.849$, $R R=0.070$

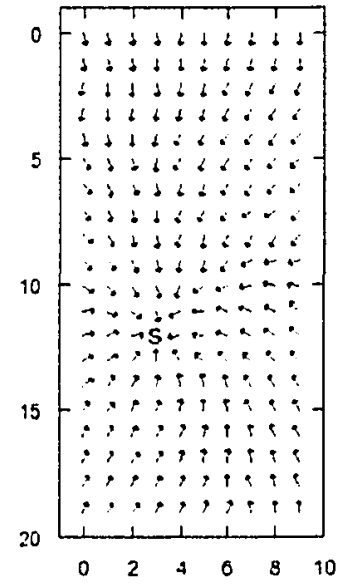

(c) SIMatch, hall1, $\mathrm{s}=(3,12)$ : $A A E=0.27 \cdot$, $R R=1.000$

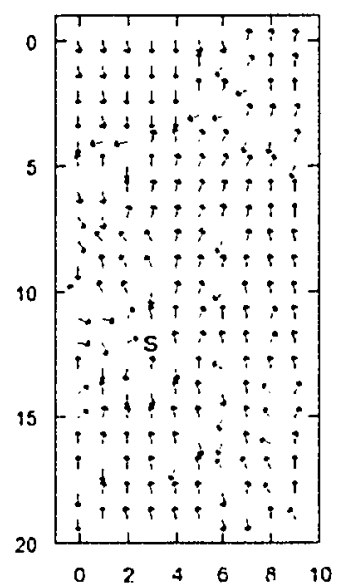

(f) SIPair, hall2, $\mathrm{s}=(3,12)$ : $A A E=1.478$, $R R=0.015$

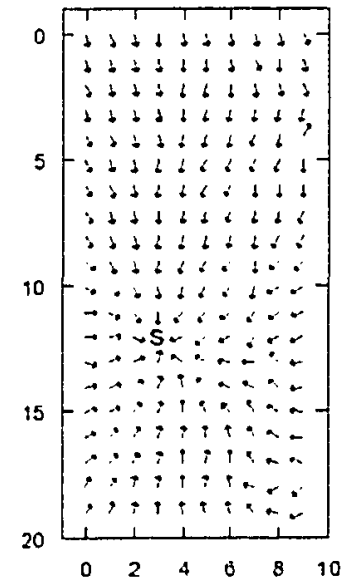

(d) SIMatch, hall2, $\mathrm{s}=(3,12):$ $A A E=0.290$, $R R=0.975$

Figure 7.7: Home vector fields for Warping $(a, b)$, SIMatch $(c, d)$, and SIPair $(e, f)$ for snapshot position $(3,12)$ in image collections hall1 $(\mathrm{a}, \mathrm{c}, \mathrm{e})$ and hall2 $(\mathrm{b}, \mathrm{d}, \mathrm{f})$. 


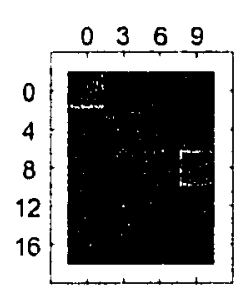

(a) Warping: $A A E_{*}=$ $0.295 \pm 0.151$

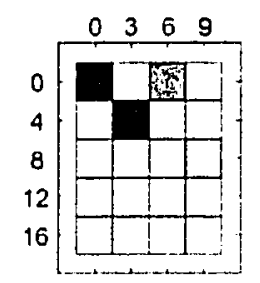

(d) Warping:

$R R_{*}=$

$0.841 \pm 0.289$

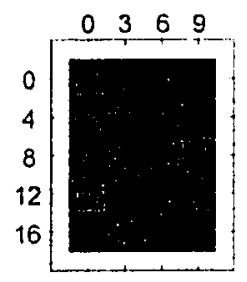

(b) SIMatch: $A A E_{*}=$ $0.38 \mathrm{~T} \pm 0.101$

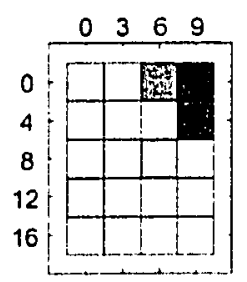

(e) SIMatch: $R R_{*}=$ $0.855 \pm 0.265$

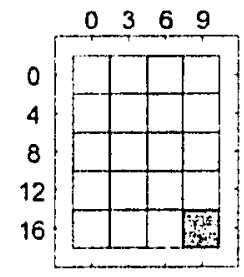

(c) SIPair:

$$
A A E_{*}=
$$

$1.84 \pm 0.267$

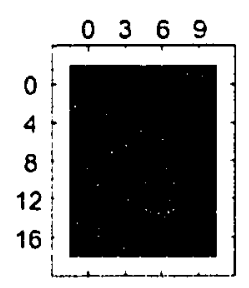

(f) SIPair:

$R R_{*}=$ $0.0455 \pm 0.045 i$

Figure 7.8: $A . A E$ and $R R$ across snapshot positions for all methods on image collection hall1. $A A E$ max. $=2.288, R R \max .=1$.

\section{Cross-Collection Tests}

Figure 7.10 plots $A A E_{*}$ and $R R$, for the cross-collection experiment. The performance characteristics of SIMatch and Warping are again broadly similar with some notable exceptions. With regards to the $A A E$ metric, sIMatch appears more insensitive to pairs of collections involving winlit. However the $R R$ plot shows that SIMatch and Warping both do poorly for pairs involving either doorlit or winlit. Also, SIMatch appears particularly sensitive to pairs that involve day. Not surprisingly, it is found that SIPair performs quite poorly for practically all combinations of image collections.

\subsection{Comparative Results}

Table 7.1 presents the result of statistical analyses comparing Warping, SIMatch, and SIPair on image collection original. It was found for both metrics that SIMatch performed significantly better than both Warping and SIPair. Also, Warping performed significantly better than SIPair in terms of the $R R$ metric. 


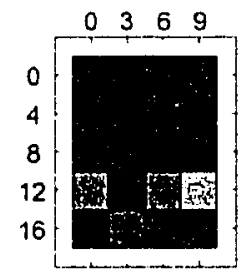

(a) Warping: $A A E_{*}=$ $0.45 \bar{T} \pm 0.185$

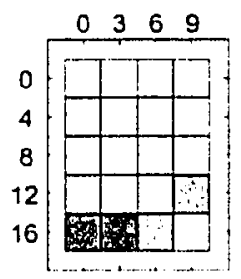

(d) Warping:

$R R_{+}=$ $0.798 \pm 0.202$

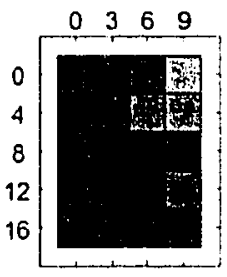

(b) SIMatch: $A A E$. $=$ $0.411 \pm 0.209$

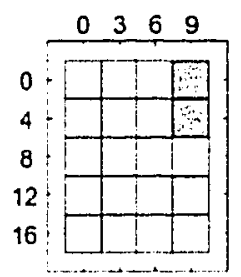

(e) SIMatch:

$R R_{*}=$ $0.914 \pm 0.148$

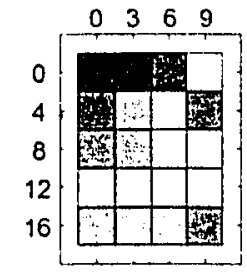

(c) SIPair: $A A E_{*}=$ $1.22 \pm 0.435$

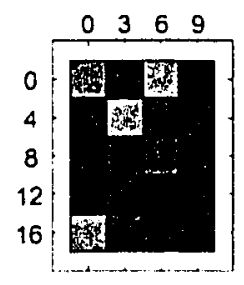

(f) SIPair:

$R R_{\bullet}=$

$0.151 \pm 0.151$

Figure 7.9: $A A E$ and $R R$ across snapshot positions for all methods on image collection hal12. $A A E \max .=2.12 \bar{\gamma}, R R \max .=1$.

Tables 7.2 and 7.3 present results of the same tests for image collection hall1 and hal12, respectively: In fact the same results were found for both metrics and in both image collections. The only significant differences were that both Warping and SIMatch performed significantly better than SIPair.

Statistical analysis of the cross-collection tests is reported in table 7.4. As above, it was found that SIPair performed significantly worse than the other two methods. Also it was found that Warping performed significantly better than SIMatch in $R R$, but not significantly different in $A A E$.

In summary, SIMatch performed equivalently or better than Warping for all tests except the cross-collection tests. Meanwhile, SIPair performed equivalently to the other two methods only in terms of $A A E$, and only on image collection original. 


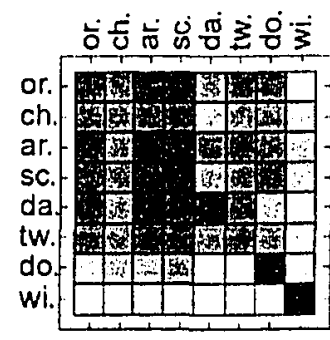

(a) Warping: $A A E_{*}(*, *)=$ $0.836 \pm 0.361$

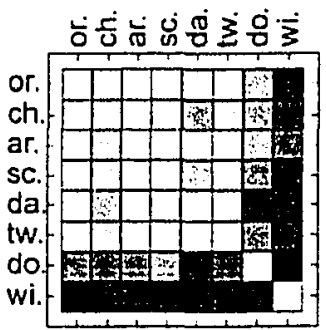

(d) Warping:

$R R_{*}(*, *)=$ $0.551 \pm 0.251$

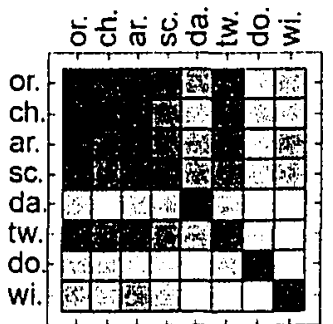

(b) SIMatch: $A A E_{*}(*, *)=$ $0.773 \pm 0.324$

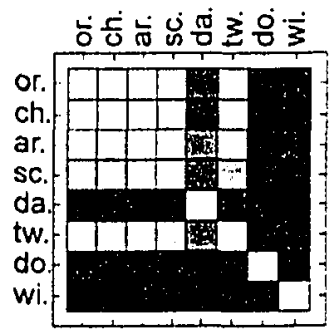

(e) SIMatch:

$$
R R_{*}(*, *)=
$$$$
0.444 \pm 0.332
$$

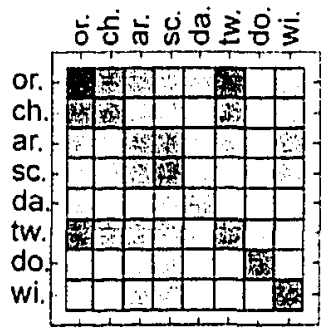

(c) SIPair: $A A E_{*}(*, *)=$ $1.06 \pm 0.196$

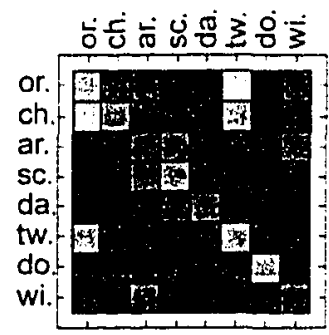

(f) SIPair:

$R R_{*}(*, *)=$ $0.203 \pm 0.126$

Figure 7.10: $A A E_{*}$ and $R R_{*}$ over all pairings of the eight office image collections. See figure 4.9 for notation. $A A E$ max. $=1.811, R R$ max. $=1$.

Significance of difference in $A . A E_{*}$ (original)

\begin{tabular}{|l|l|l|l|}
\hline & Warping & SIMatch & SIPair \\
\hline Warping & $\mathrm{X}$ & & \\
\hline SIMatch & $* * *$ & $\mathrm{X}$ & $* *$ \\
\hline SIPair & & & $\mathrm{X}$ \\
\hline
\end{tabular}

Significance of difference in $R R_{*}$ (original)

\begin{tabular}{|l|l|l|l|}
\hline & Warping & SIMatch & SIPair \\
\hline Warping & $\mathrm{X}$ & & $* *$ \\
\hline SIMatch & $*$ & $\mathrm{X}$ & $* * *$ \\
\hline SIPair & & & $\mathrm{X}$ \\
\hline
\end{tabular}

Table 7.1: Statistical significance of the difference in $A A E_{*}$ (original) and $R R_{*}$ (original) between homing methods. See table 4.1 for notation. 
Significance of difference in $A A E_{*}$ (hall1)

\begin{tabular}{|l|l|l|l|}
\hline & Warping & SIMatch & SIPair \\
\hline Warping & $\mathrm{X}$ & & $* * *$ \\
\hline SIMatch & & $\mathrm{X}$ & $* * *$ \\
\hline SIPair & & & $\mathrm{X}$ \\
\hline
\end{tabular}

Significance of difference in $R R_{*}$ (hall1)

\begin{tabular}{|l|l|l|l|}
\hline & Warping & SIMatch & SIPair \\
\hline Warping & $\mathrm{X}$ & & $* * *$ \\
\hline SIMatch & & $\mathrm{X}$ & $* * *$ \\
\hline SIPair & & & $\mathrm{X}$ \\
\hline
\end{tabular}

Table 7.2: Statistical significance of the difference in $A . A E_{*}\left(\right.$ hall1) and $R R_{*}($ hall1) between homing methods. See table $t .1$ for notation.

Significance of difference in $A A E_{*}($ hall2)

\begin{tabular}{|l|l|l|l|}
\hline & Warping & SIMatch & SIPair \\
\hline Warping & $\mathrm{X}$ & & $* * *$ \\
\hline SIMatch & & $\mathrm{X}$ & $* * *$ \\
\hline SIPair & & & $\mathrm{X}$ \\
\hline
\end{tabular}

Significance of difference in $R R_{*}$ (hall2)

\begin{tabular}{|l|l|l|l|}
\hline & Warping & SIMatch & SIPair \\
\hline Warping & $\mathrm{X}$ & & $* *$ \\
\hline SIMatch & & $\mathrm{X}$ & $* * *$ \\
\hline SIPair & & & $\mathrm{X}$ \\
\hline
\end{tabular}

Table 7.3: Statistical significance of the difference in $A A E_{*}$ (hal12) and $R R_{*}$ (hall2) between homing methods. See table 4.1 for notation. 
Significance of difference in $A A E_{*}(*, *)$

\begin{tabular}{|l|l|l|l|}
\hline & Warping & SIMatch & SIPair \\
\hline Warping & $\mathrm{X}$ & & $* * *$ \\
\hline SIMatch & & $\mathrm{X}$ & $* * *$ \\
\hline SIPair & & & $\mathrm{X}$ \\
\hline
\end{tabular}

Significance of difference in $R R_{*}(*, *)$

\begin{tabular}{|l|l|l|l|}
\hline & Warping & SIMatch & SIPair \\
\hline Warping & $\mathrm{X}$ & $* *$ & $* * *$ \\
\hline SIMatch & & $\mathrm{X}$ & $* * *$ \\
\hline SIPair & & & $\mathrm{X}$ \\
\hline
\end{tabular}

Table 7.4: Statistical significance of the difference in $A A E_{*}(*, *)$ and $R R_{*}(*, *)$ between homing methods for all pairings of image collections from the office environment. See table 4.1 for notation.

\subsection{Analysis}

What reasons might there be for SIPair's poor performance on all image collections but original? The first suspicion was that the parameters chosen for SIPair, while optimal for original, were not the best to choose for the other collections. However, optimizing SIPair for both hall1 and hall2 produces little improvement in performance on these image collections. Therefore there must be some aspect of these environments which makes SIPair fundamentally unworkable within them.

The most salient difference between the hall collections and original is the depth of viewed objects. The main hall of Bielefeld university is hundred's of metres in length whereas the robotics lab where original was captured is only 8.25 metres in its longest dimension. In the plots of $D P(\mathbf{p}, \mathbf{p})$ shown in figure 7.4 the peaks of activity corresponded reasonably well with the locations of the FOC and FOE. This is because the FOC and FOE were the only points of low flow within the image. However, if there are distant objects in the scene then they will present as low flow regions as well (this topic is discussed in chapter 2). Figure 7.11 shows snapshot image $(3,12)$ and current image $(0,12)$ selected from image collection hall1. The figure also shows a plot of $D P(\mathbf{p}, \mathbf{p})$ for this situation. High activity is found at the FOC, however there are high values found throughout the rest of the image as well. The peak value occurs at position $(1,27)$, far away from the FOC. It can be observed that this position corresponds to the far end of the hall in both images. 
Snapshot
Image $(3,12)$

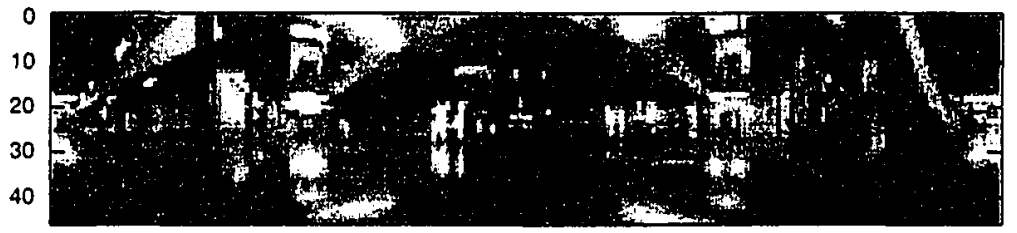

Current

Image $(0,12)$

$D P(\mathbf{p}, \mathbf{p})$ at

$(0,12)$
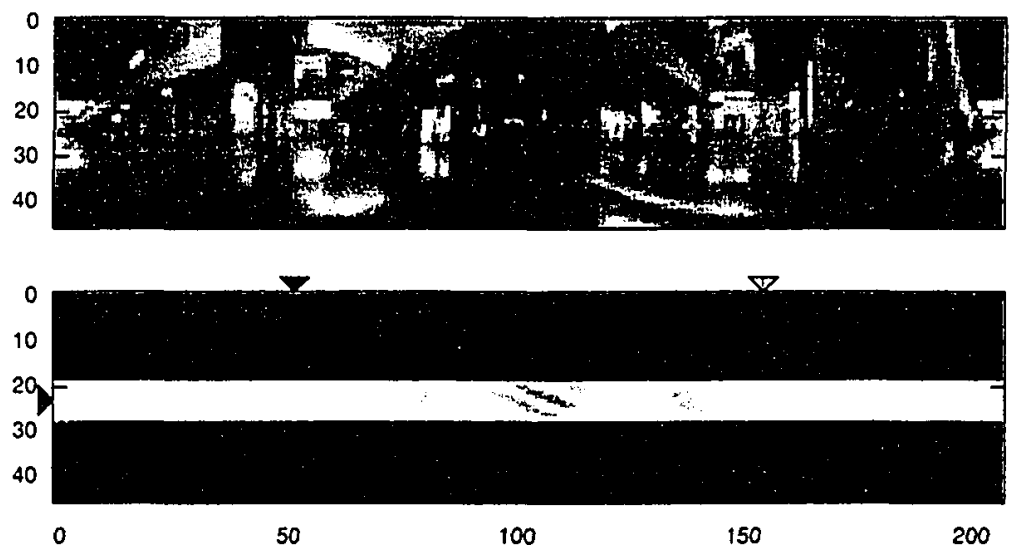

Figure 7.11: Dot product image (c) for snapshot position $(3,12)$ and current position $(0,12)$, of image collection hall1. Hence, the correct FOC should be around $(52,23)$ while the correct FOE should be near $(155,23)$. These positions are indicated in (c) as in figure 7.3 . The location of maximum $D P(\mathbf{p}, \mathbf{p})$ is indicated by an ' $\mathrm{X}$ '.

As argued by Cartwright and Collett [7] some form of filtering scheme might be useful to remove distant objects from the scene to allow more precise visual homing. Unfortunately, incorporating a filter for distant objects into SIPair is problematic. If a distant object exists in a direction $\theta$ then filtering that object out leaves a hole in the dot product image. If one of the foci then happens to be at or around $\theta$ then the hole will overlap the foci, which will therefore go undetected. If both foci happen to be overlapped by such holes then the method will fail completely.

Another unrelated problem with SIPair is its poor performance on the cross-collection tests. However, given the method's existing difficulties as described above, the poor performance on the cross-collection tests was not further investigated.

Meanwhile, SIMatch does require some investigation. The primary motivation for this investigation is not to understand where and why it fails, but whether its existing level of success can be maintained if the method is simplified. As will be shown below in section 7.10 SIMatch has high computational complexity and generally low biological plausibility: If no simpler variation of this method can be found then it may be of interest to the technical 
community, but not likely to the biological community.

A first step in determining whether SIMatch can be simplified is to test it with lower cost parameter settings. We can divide the method's parameters into those controlling the search process, and those controlling the image descriptor. For the moment we concentrate on the image descriptor. The most pertinent parameters which control the complexity of the image descriptor are the length of the descriptor vector, $n$, and the length of channels to sum over, $l_{\max }$. The search for optimal parameters in section 7.6 set $n=32$ and $l_{\max }=50$. Figure 7.12(a) varies the parameter $n$ in factors of 2 from 1 to 64 . The figure plots $A A E_{*}$ (original) for each value. It can be observed that $A A E$ decreases with increasing $n$ but then levels off to a stable value at $n=32$. This makes sense because if the descriptors are longer then they contain more information. Thus, there should be less ambiguity between them, and therefore fewer incorrect correspondences. The error curve becomes asymptotic because increasing the descriptor's length beyond a certain limit (here $n=32$ ) adds no new information.

A much more unexpected result comes in varying the length of channels to sum over. Figure 7.12(b) plots this length, and the resulting value of $A A E_{*}$ (original). Quite surprisingly; this parameter has very little impact. The minimum error occurs at $l_{\max }=40$ but even for very low values of $l_{\max }$ the error remains almost constant. The length of channels to sum over controls the effective radius of the scale invariant image descriptor. Small radii ought to make the descriptor less invariant to scale changes. Yet even when the length of channels is reduced to one, the value of $A A E$ remains relatively low. If the length of channels is one then the descriptor is doing nothing more then capturing a vector of pixel values for adjacent points. Thus, we can only conclude that this descriptor's property of scale invariance is actually not crucial to the success of SIMatch.

If the scale invariance property is not critical then a simpler image descriptor which just represents local pixel values might suffice for homing. The test of this hypothesis appears in the next chapter where the simple method of block matching is applied to visual homing.

\subsection{Complexity and Biological Plausibility}

\subsubsection{The Scale Invariant Descriptor}

The computational cost of computing the image descriptor $\mathbf{g}$ is given by the product of the descriptor length $n$, and the length of chamnels $l_{\max }$. The unit of this cost is pixel operations. In order to go from the descriptor $\mathbf{g}$ to the scale invariant descriptor $\mathbf{h}$ we must 


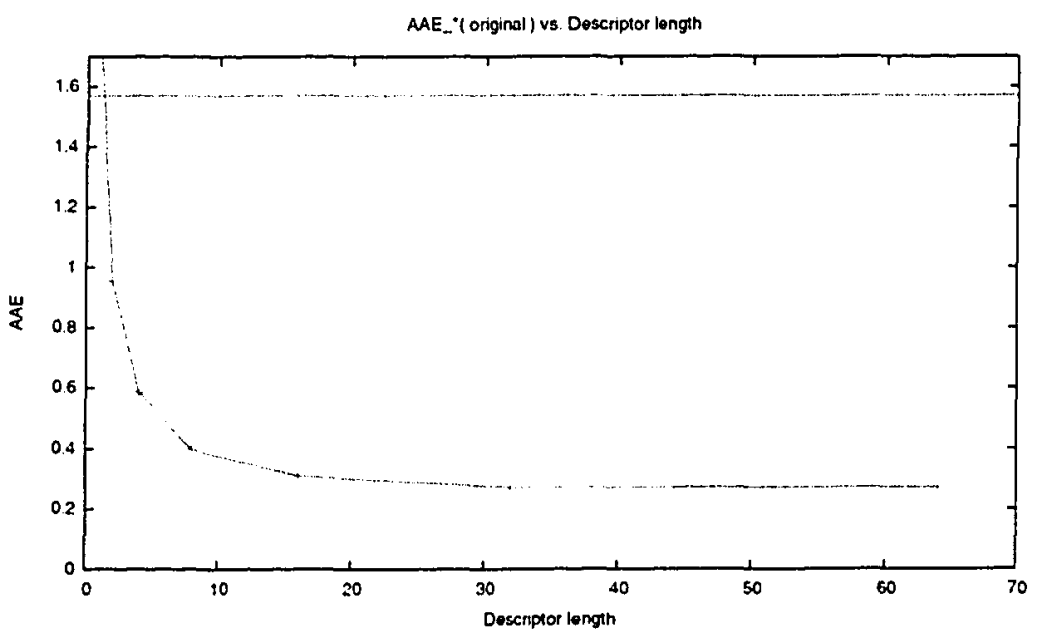

(a)

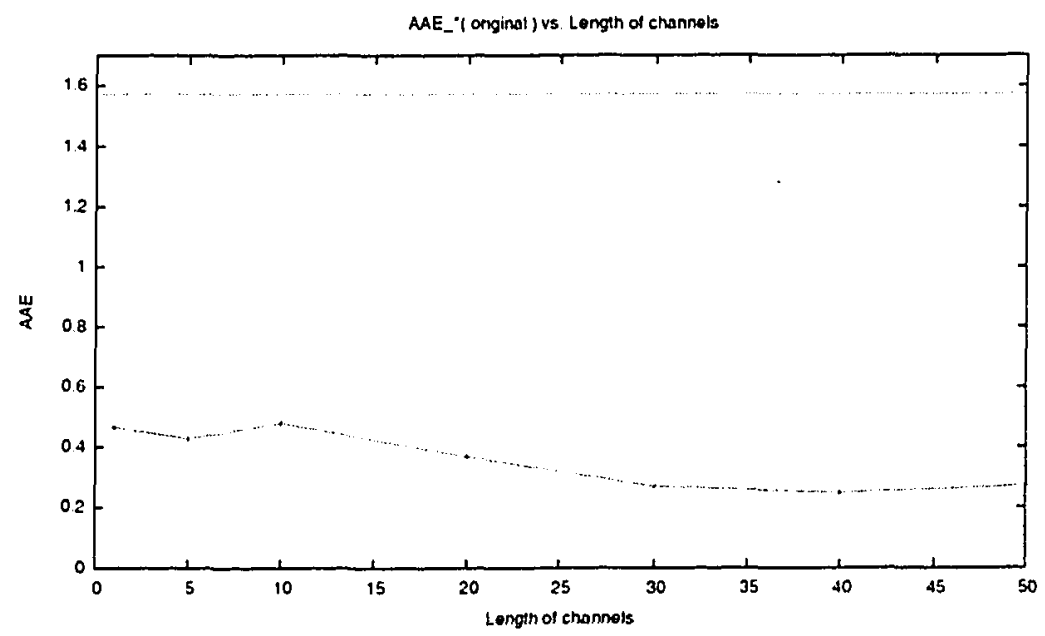

(b)

Figure 7.12: Plots of $A A E_{*}$ (original) vs. parameters. A dashed line is shown at $\pi / 2$. 
normalize $g$. This costs approximately $3 n$ pixel operations ${ }^{1}$. Therefore the cost per scale invariant image descriptor is

$$
\mathcal{C}_{\text {descriptor }}=n\left(l_{\max }+3\right)
$$

\subsubsection{SIMatch}

Scale invariant descriptors must first be computed for all origin points and all candidate points in the snapshot and current images.

The number of origin points in the snapshot image is $n_{x} n_{y}$ while there are $w h$ candidate points in the current image ${ }^{2}$. The cost of computing scale invariant image descriptors for all candidate points in the current image is therefore

$$
\mathcal{C}_{\text {descriptor }} \text { wh }
$$

SIMatch requires a search for each correspondence. To compare two scale invariant image descriptors the dot product is computed. This requires $n+1$ operations. The dot product is computed between an origin point and all candidate points within a radius of $q$. Thus the total cost for each correspondence search is

$$
\mathcal{C}_{\text {search }}=(n+1)(2 q+1)^{2}
$$

Thus, the total number of operations required to compute a home vector is

$$
\mathcal{C}_{\text {STMatch }}=\mathcal{C}_{\text {descriptor }} \text { wh }+\mathcal{C}_{\text {search }} n_{x} n_{y}
$$

Note that this does not include pre-processing, vector mapping, or the summation required to compute the final home vector. For the parameters selected in section 7.6 the quantity above comes to approximately 89 million. On a Pentium $42 \mathrm{Ghz}$ PC it takes an average of 1.7 seconds to compute a home vector. Using the rough assumptions posited in section 1.4.2 that all low-level operations require a single neurons, and all processing occurs in parallel, we conclude that the implementation of SIMatch would require 89 million neurons. Thus, it seems highly implausible that this sort of algorithm could be implemented in the limited neural hardware of an insect.

\footnotetext{
${ }^{1}$ Asymptotic notation is not employed as we wish to retain constants and make some numerical comparisons between methods. Numerical comparisons are necessary as the methods compared generally do not share parameter settings

${ }^{2}$ In fact, it is less computationally expensive to compute the larger number of points $(w h)$ for the snapshot image. This topic was discussed in section 6.5.8. If we reverse the roles of origin and candidate points in this manner (and reverse the home vector) then we need only compute $n_{r} n_{y}$ candidate points for each current image. Nevertheless, the analysis proceeds with the assumption that the larger number of points is computed for the current image.
} 


\subsubsection{SIPair}

Unlike SIMatch, SIPair does not involve a search process. Only image descriptors at the same retinotopic position are compared. However, equation (7.22) incurs a non-trivial cost just to compute the image descriptors. Thus, while SIPair satisfies the constraints for retinotopic processing, we proceed to analyze its complexity.

Scale invariant descriptors must first be computed for all points in both the snapshot and current images. However, only those descriptors lying within a window of height $h_{h}$ along the horizon are needed. This makes $h_{h}$ the effective height of the image. The cost of computing scale invariant image descriptors for the current image within the required region is therefore

$$
\mathcal{C}_{\text {descriptor }} \text { wh }
$$

The dot product of descriptors at the same image position must be calculated. As mentioned above, the cost of the dot product operation is $n+1$. Therefore the total cost to compute the dot product image is

$$
\left(\mathcal{C}_{\text {descriptor }}+n+1\right) w h_{h}
$$

Once the dot product image is computed the maximum value is extracted and used to compute the home vector. These are relatively low-cost operations which are not included here. For the parameters selected in section 7.6 the quantity above comes to approximately. 3 million and it takes an average of 1.1 seconds to compute a home vector. Thus, using the same reasoning as above, SIPair does not appear very biologically plausible.

\subsection{Conclusions}

This chapter has described a new scale invariant image descriptor as well as two different homing methods based on this descriptor. The search-based method SIMatch exhibits performance that is competitive with Warping in most cases. SIPair, while designed to satisfy' the constraints of retinotopic processing, fails to be competitive with Warping. Both methods have low biological plausibility because of the high computational cost of computing image descriptors.

Nevertheless, this chapter has yielded two distinct accomplishments. Firstly; the image descriptor is able to capture something about the local pattern of edges in an image such that the impact of scale changes is minimized. Thus, this descriptor may be of interest to the general computer vision community: Similar scale invariant image descriptors such as Lowe's [66] have a more complex structure. Secondly; the analysis of SIMatch revealed 
that this scale-invariant property was not crucial for homing success. This points the way towards simpler methods that operate on less information-rich image descriptors. This line of inquiry is pursued in the next chapter. 


\section{Chapter 8}

\section{Towards Simpler Matching Methods}

\subsection{Introduction}

In the previous chapter a homing method based on matching scale-invariant image descriptors was presented. This method, SIMatch, was the first method presented in this thesis which exhibited performance competitive with Warping for almost all environments. Further, it was found to maintain good performance even when configured with very 'light' parameters that significantly reduced the information represented by the image descriptor. This result prompts the question: What is the minimal image descriptor that can can be matched between the snapshot and current images which still affords competitive homing? This chapter answers that question.

Ostensibly, this direction of research is a digression from the realm of homing algorithms relevant to biology. All of the methods presented in this chapter require a search process to find appropriate matches between image descriptors. However, as we move in the direction of simpler image descriptors a new technique for estimating correspondences will be developed. This new technique will be presented in the next chapter and will return us to a more biologically relevant course.

This chapter begins with the classical optic flow technique of block matching [49]. Block matching takes a block of pixels from one image and searches for the best matching block in another image within a fixed search radius. The degree of match between blocks is usually judged by the Sum of Squared Differences (SSD) or some other local correlation measure. Variants on block matching are widely used in computer vision to determine the motion 
between frames of a video sequence so as to allow more efficient encoding. Experiments presented here reveal block matching to be highly successful on both offline images and for online control of a mobile robot.

An analysis of block matching's success leads to the proposal of two variants, based on matching much simpler image descriptors. These two methods are carried forward into the next chapter which adapts them into a less computationally expensive and more biologically plausible framework. Note that both this chapter and the next describe joint work by Vardy and Möller [98].

\subsection{BlockMatch}

Assume we have a snapshot image $S$, a current image $C$, and we wish to find correspondences between these two images. We further assume that these two images were captured at the same compass orientation. Correct correspondence is achieved if for each origin point $\mathbf{p}$ in $S$ we can find an appropriate candidate point $\mathbf{p}^{\prime}$ in $C$ such that the same image features appear in the local regions surrounding these two points. The method BlockMatch operates by doing a search for each $\mathrm{p}$ in $S$ to find the best matching region in $C$. A standard means of judging the quality of a match is to calculate the Sum of Squared Differences (SSD)

$$
\operatorname{SSD}\left(\mathbf{p}, \mathbf{p}^{\prime}\right)=\sum_{i=-r}^{r} \sum_{j=-r}^{r}\left[S\left(p_{x}+i, p_{y}+j\right)-C\left(p_{x}^{\prime}+i, p_{y}^{\prime}+j\right)\right]^{2}
$$

with $i, j \in \mathbb{Z}$. A low value of SSD indicates a good match. The quantity $r$ is the block radius. Large values of $r$ require regions to match over a large area and have increased computational cost. However, it will turn out that a relatively small $r$ is sufficient for visual homing.

As for SIMatch, a correspondence search is carried out by BlockMatch not for every position in $S$ but only for a sampling of positions at integer multiples of $m_{x}$ in the horizontal direction and $m_{y}$ in the vertical direction, where $m_{x}$ and $m_{y}$ are also integers. Again, for images of width $w$ pixels and height $h$ pixels the number of horizontal and vertical sampling points is as follows:

$$
\begin{aligned}
& n_{x}=\left\lfloor w / m_{x}\right\rfloor \\
& n_{y}=\left\lfloor h / m_{y}\right\rfloor
\end{aligned}
$$

Thus, the total number of correspondence searches is $n_{x} n_{y}$. Each search involves computing the SSD for all positions in $C^{\prime}$ within a radius of $q$ pixels of $\mathbf{p}$, and selecting $\check{\mathbf{p}}$ as the candidate 
match with the lowest SSD:

$$
\begin{gathered}
\check{\mathbf{p}}=\arg \min _{\mathbf{p}^{\prime} \in E_{\eta}(\mathbf{p})} \operatorname{SSD}\left(\mathbf{p}, \mathbf{p}^{\prime}\right) \\
E_{q}\left(\left(p_{x}, p_{y}\right)\right)=\left\{\left(p_{x}+i, p_{y}+j\right)|i, j \in \mathbb{Z},| i|\leq q \wedge| j \mid \leq q\right\}
\end{gathered}
$$

Also, as was the case for CGSM and SIMatch we add the constraint that origin points $\mathbf{p}$ in the snapshot image will only be paired with candidate points in the current image $\mathbf{p}^{\prime}$ which are on the same side of the horizon.

The position $\check{\mathrm{p}}$ with the lowest SSD is used to compute the correspondence vector $\delta$

$$
\delta=\left(\begin{array}{l}
\delta_{x} \\
\delta_{y}
\end{array}\right)=\left(\begin{array}{c}
\Delta_{x}\left(\check{p}_{x}-p_{x}\right) \\
\Delta_{y}\left(\check{p}_{y}-p_{y}\right)
\end{array}\right)
$$

where $\Delta_{x}$ represents the inter-pixel angle in the horizontal direction and $\Delta_{y}$ represents vertical inter-pixel angle.

From each of these correspondence vectors a home vector is determined using the exact vector mapping method from chapter 5 . These home vectors are then summed and normalized to produce the method's final home vector.

\subsubsection{Pre-Processing}

In its typical application within computer vision, block matching is applied directly without any image pre-processing. In the parameter search described below the option of no preprocessing was available. Also available is the use of a Sobel filter to enhance edges and the use of a Gaussian low-pass filter. The number of applications of the Gaussian filter is left as a parameter.

Also, as was observed in the previous chapter, the top and bottom portions of the image tend to exhibit less distinguishing features than regions near the horizon. Therefore, one of the parameters below controls how many of the top and bottom rows to exclude from the matching process.

\subsubsection{Optimizing Parameters}

The parameters tested for BlockMatch are as follows:

- Number of applications of Gaussian filter: $0,1,2,3$, or 4 .

- Use Sobel filter: true or fals?. 
- Number of top and bottom image rows to exclude: 0,5 , or 10 .

- Search radius, $q: 5,10,30$, or 50 .

The best parameters found are given above in bold. The block radius parameter, $r$ was set to 1 . The step size parameters of $m_{x}$ and $m_{y}$ were not varied in this search but were set to a moderate value of $m_{x}=m_{y}=4$.

\subsubsection{Results on Image Database}

\section{Image Collection original}

Figure 8.1 shows the home vectors generated by BlockMatch and Warping on image collection original for snapshot positions $(6,4)$ and $(0,16)$. Both methods perform well at position $(6,4)$ but at position $(0,16)$ it is apparent that BlockMatch performs much better.

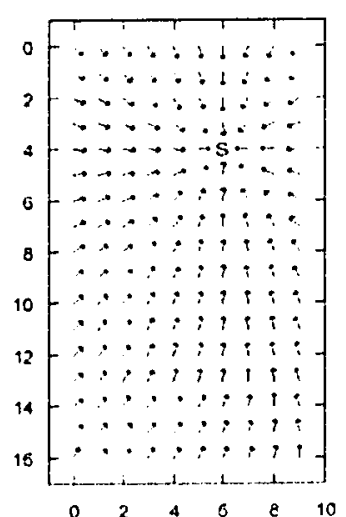

(a) Warping, $\mathrm{s}=(6,4)$ $A . A E=0.119$ $R R=1.000$

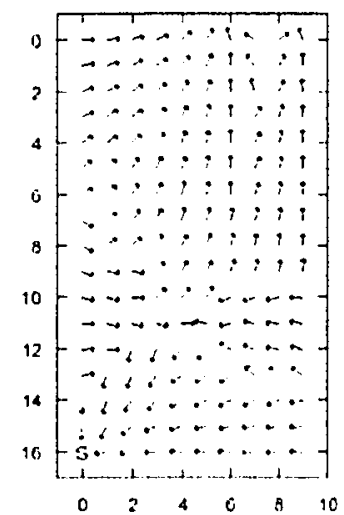

(b) Warping, $\mathrm{s}=(0,16)$ : $A A E=1.758$, $R R=0.266$

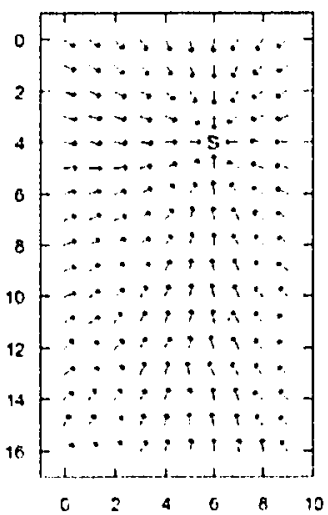

(c) BlockMatch, $s=(6,4):$ $A A E=0.165$, $R R=1.000$

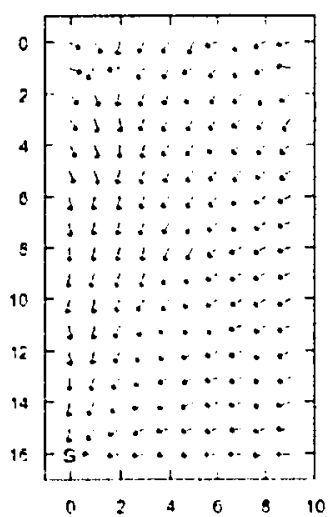

(d) BlockMatch, $\mathbf{s}=(0,16):$ $A A E=0.196$, $R R=1.000$

Figure 8.1: Home vector fields for Warping (a,b) and BlockMatch (c,d), for snapshot positions $(6,4)(a, c)$ and $(0,16)(b, d)$ in image collection original.

Figure 8.2 shows the computed $A A E$ and $R R$ for Warping and BlockMatch over twenty snapshot positions (also shown are the same plots for two methods presented later in this chapter). For both performance metrics BlockMatch performs extraordinarily well. In fact, for the $R R$ metric it performs perfectly-achieving a return ratio of one for every snapshot position. 


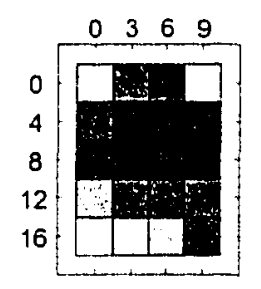

(a) Warping: $A A E_{*}=$ $0.576 \pm 0.461$

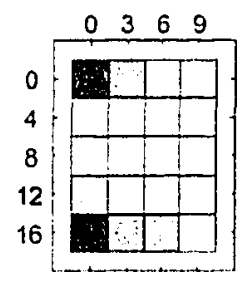

(e) Warping:

$R R_{*}=$ $0.792 \pm 0.226$

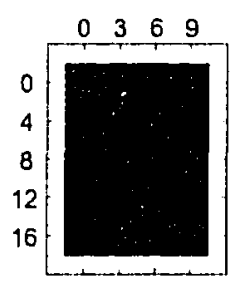

(b) BlockMatch: $A A E$, $=$ $0.173 \pm 0.0468$

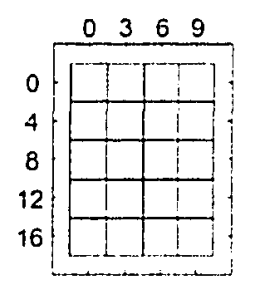

(f) BlockMatch: $R R_{*}=1 \pm 0$

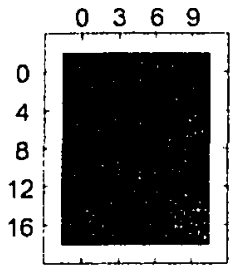

(c) IntMatch: $A . A E_{*}=$ $0.27 \pm 0.0678$

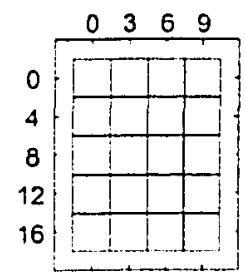

(g) IntMatch: $R R_{*}=$ $0.997 \pm 0.0146$

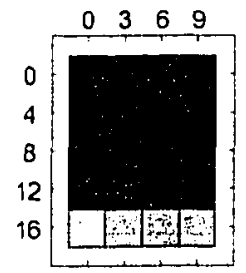

(d) GradMatch: $A A E_{*}=$ $0.349 \pm 0.316$

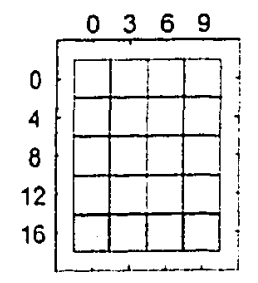

(h) GradMatch: $R R_{*}=$ $0.932 \pm 0.14$

Figure 8.2: $A A E$ and $R R$ across snapshot positions for all methods on image collection original. Beneath each plot is shown the mean value of the metric ( $A A E_{*}$ or $R R_{*}$ ) and its standard deviation (the number following the ' \pm ' symbol). Average values for $A A E \max .=1.758, R R \max .=1$.

\section{Image Collections hall1 and hall2}

Figure 8.3 shows home vectors generated by Warping and BlockMatch on the hall image collections at snapshot position $(3,12)$. Both methods yield generally appropriate home vector fields. However, there are a number of incorrect vectors embedded in the vector field for Warping in hall2. Errors in BlockMatch's vector field are scarcely noticeable.

Figure 8.4 shows the computed $A A E$ and $R R$ across all twenty snapshot for both methods on image collection hall1. Again, BlockMatch's performance exceedingly high as mensured by both performance metrics. Figure 8.5 shows that the same holds true for image collection hall2.

\section{Cross-Collection Tests}

Figure 8.6 plots $A A E_{*}$ and $R R_{*}$ for the cross-collection experiment. The performance of BlockMatch remains strong, although like Warping it performs relatively poorly for pairs of 


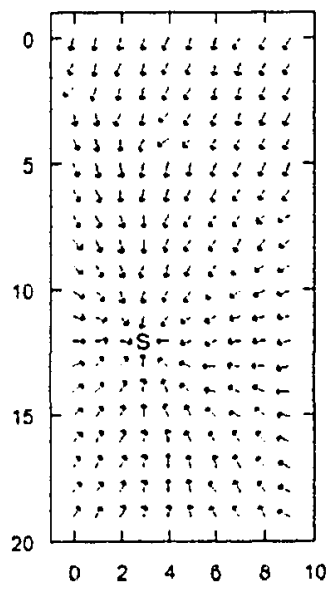

(a) Warping, hall1, $\mathrm{s}=(3,12)$ : $A . A E=0.160$, $R R=1.000$

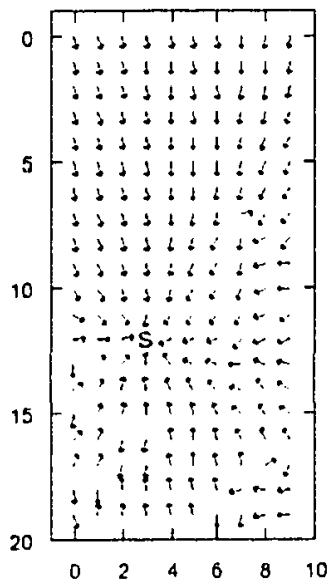

(b) Warping, hal12, $\mathrm{s}=(3,12)$ : $A A E=0.40 \%$, $R R=0.915$

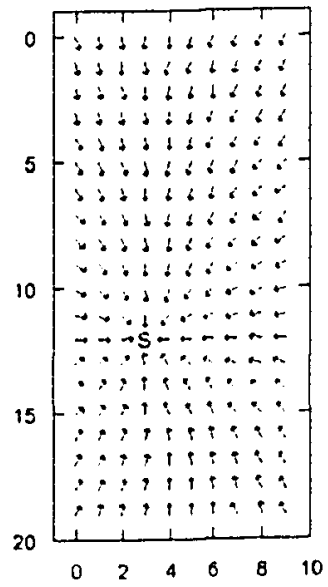

(c) BlockMatch, hall1, $\mathrm{s}=(3,12)$ : $A A E=0.144$, $R R=1.000$

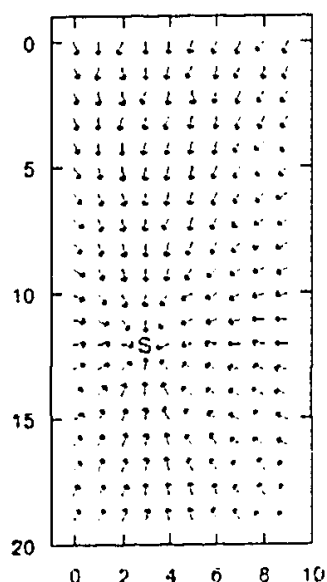

(d) BlockMatch. hall2, $\mathbf{s}=(3,12)$ : $A . A E=0.114$, $R R=1.000$

Figure 8.3: Home vector fields for Warping $(a, b)$ and BlockMatch $(c, d)$, for snapshot. position $(3,12)$ in image collections hall1 (a,c) and hall2 (b,d).

image collections that include doorlit or winlit. Otherwise, BlockMatch appears quite robust to environmental modifications.

\subsubsection{Comparative Results}

As described in section 3.3.5, we compare the $A A E$ of two homing methods with a paired t-test, and the $R R$ of two methods with a Wilcoxon matched pairs test. The results of these tests on Warping and BlockMatch for all four experimental conditions are shown in table 8.1. In all cases but one, BlockMatch performs significantly better than Warping. The one exception occurs for the $R R$ metric on image collection original. In this image collection BlockMatch achieves a perfect return ratio of one for all snapshot positions. Thus, the standard deviation is zero and statistical tests are inappropriate. It seems safe to conclude, however, that for all tests BlockMatch outperforms Warping. 


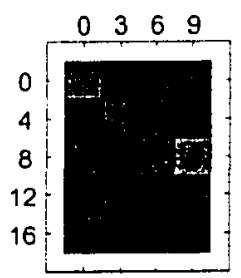

(a) Warping:

$A A E_{*}=$

$0.295 \pm 0.151$

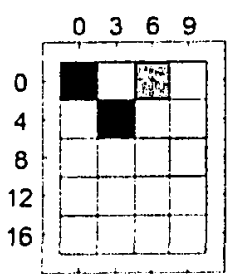

(e) Warping:

$R R_{*}=$

$0.841 \pm 0.289$

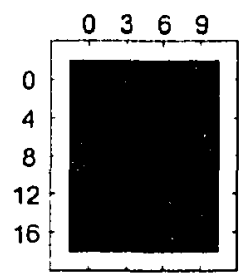

(b) BlockMatch:

$A A E_{*}=$

$0.174 \pm 0.0528$

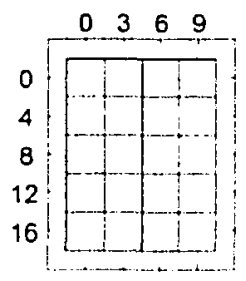

(f) BlockMatch:

$R R_{*}=$

$0.999 \pm 0.002 .47$

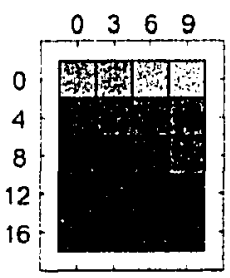

(c) IntMatch:

$A A E$, =

$0.545 \pm 0.281$

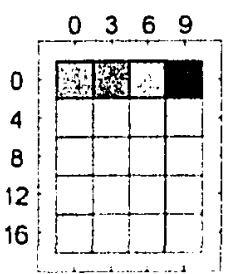

(g) IntMatch:

$R R_{+}=$

$0.825 \pm 0.243$

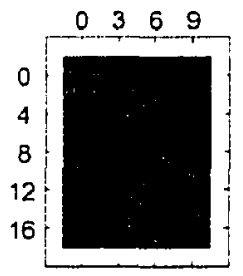

(d) GradMatch:

$A A E_{*}=$ $0.268 \pm 0.0715$

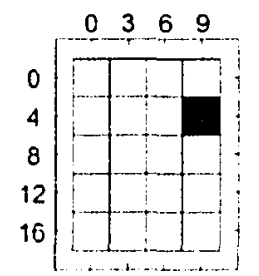

(h) GradMatch:

$R R_{+}=$ $0.955 \pm 0.198$

Figure 8.4: $A A E$ and $R R$ across snapshot positions for all methods on image collection hall1. $A A E$ max. $=2.288, R R$ max. $=1$.

Significance of difference in $A A E_{*}$

\begin{tabular}{|l|l|l|l|l|}
\hline & original & hall1 & hall2 & cross \\
\hline Warping & & & & \\
\hline BlockMatch & $* * *$ & $* * *$ & $* * * *$ & $* * * *$ \\
\hline
\end{tabular}

Significance of difference in $R R$,

\begin{tabular}{|l|l|l|l|l|}
\hline & original & hall1 & hall2 & cross \\
\hline Warping & & & & \\
\hline BlockMatch & NA & $* * *$ & $* * *$ & $* * * *$ \\
\hline
\end{tabular}

Table 8.1: Statistical significance of the difference in $A A E_{*}$ and $R R_{*}$ between Warping and BIockMatch. Significance for each cell is indicated if the corresponding method is significantly better (lower $A A E$ or higher $R R$ ) than the other method. Empty fields indicate no significant difference. For $A A E$ a paired t-test was used. For $R R$ the Wilcoxon matched pairs test was used. Legend: ${ }^{*}=(p<0.05),{ }^{* *}=(p<0.01),{ }^{* * *}$ $=(p<0.001), * * * *=(p<0.0001), \mathrm{NA}=$ not applicable (see text) 


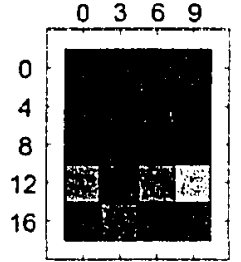

(a) Warping: $A A E_{*}=$ $0.45 T \pm 0.185$

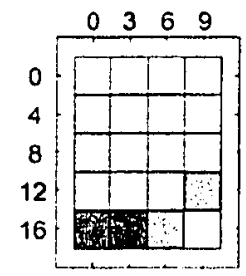

(e) Warping:

$R R=$ $0.798 \pm 0.202$

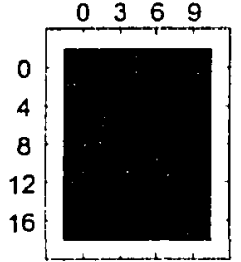

(b) BlockMatch: $A A E_{*}=$ $0.176 \pm 0.0383$

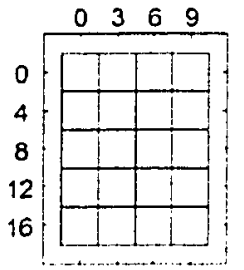

(f) BlockMatch: $R R_{\text {. }}=$ $0.998 \pm 0.007$

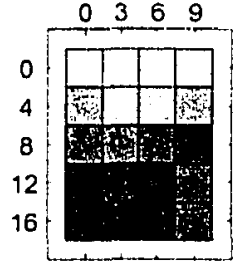

(c) IntMatch: $A A E_{*}=$ $0.873 \pm 0.415$

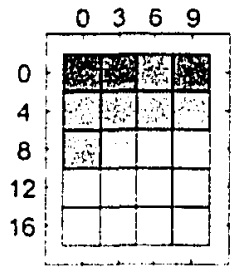

(g) IntMatch: $R R_{\text {. }}=$ $0.66 \pm 0.214$

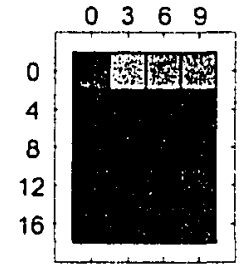

(d) GradMatch: $A A E_{\text {. }}=$ $0.461 \pm 0.211$

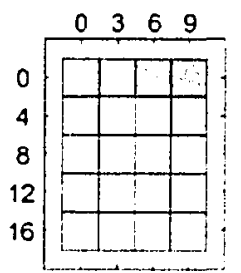

(h) GradMatch: $R R$. = $0.903 \pm 0.155$

Figure 8.5: $A A E$ and $R R$ across snapshot positions for all methods on image collection hal12. $A A E$ max. $=2.12 \overline{\mathrm{i}}, R R \max .=1$.

\subsubsection{Robot Homing Trials}

A series of online homing trials was carried out in order to test the real-world applicability of our results on BlockMatch.

Figure 8.7 shows traces of the robot homing according to BlockMatch ${ }^{1}$. For comparison, see the traces of Warping and CGSM homing in figure 6.13. The number of successful homing attempts for BlockMatch is 24 out of 24 . By contrast, the number successful for Warping was 20 out of 24 . While no definitive statements can be made from this data alone, these results do lend credence to the results obtained on the image database. That is, the superior performance of BlockMatch does not appear to be an artefact of the offline testing methodology:

\footnotetext{
${ }^{1}$ These robot homing trials were carried out on an older variant of BlockMatch which emploved a different (generally less successful) configuration.
} 


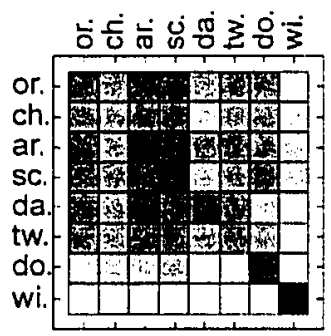

(a) Warping: $A A E_{*}(*, *)=$ $0.836 \pm 0.361$

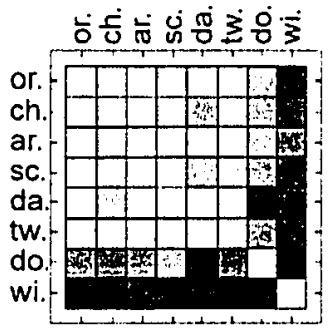

(e) Warping:

$R R_{*}(*, *)=$

$0.551 \pm 0.251$

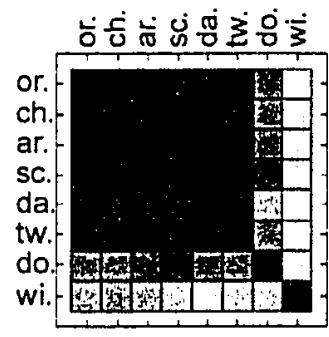

(b) BlockMatch: $A A E_{*}(*, *)=$ $0.492 \pm 0.36$

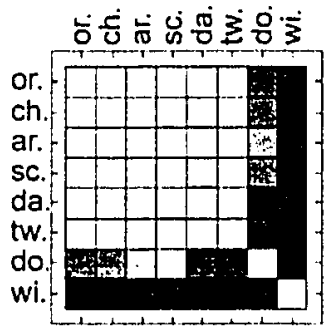

(f) BlockMatch: $R R_{*}(*, *)=$ $0.648 \pm 0.386$

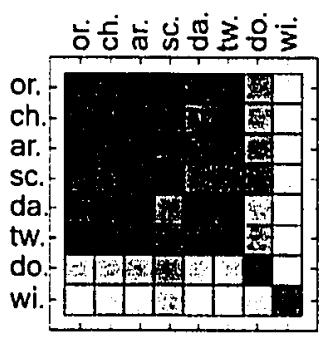

(c) IntMatch: $A . A E_{*}(*, *)=$ $0.621 \pm 0.358$

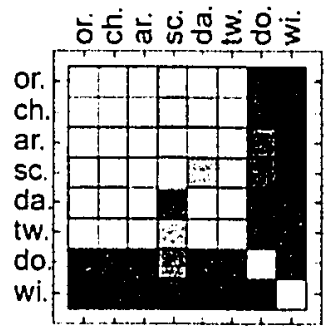

(g) IntMatch: $R R_{*}(*, *)=$ $0.555 \pm 0.382$

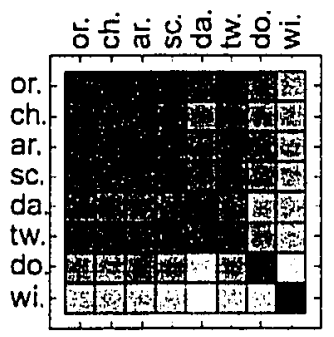

(d) GradMatch: $A A E_{+}(*, *)=$ $0.556 \pm 0.203$

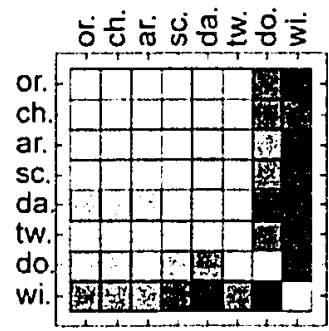

(h) GradMatch: $R R .(*, *)=$ $0.649 \pm 0.28 \mathrm{~s}$

Figure 8.6: $A A E_{*}$ and $R R_{*}$ over all pairings of the eight office image collections. See figure 4.9 for notation. $A . A E$ max. $=1.811, R R$ max. $=1$.
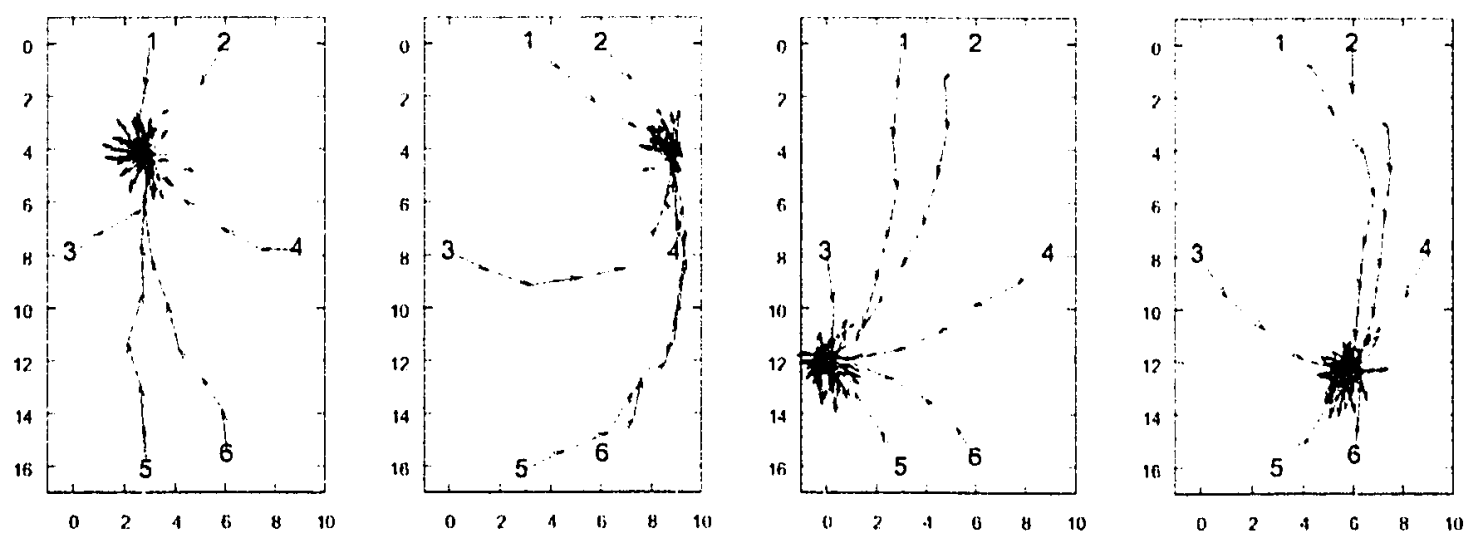

Figure 8.7: Performance of BlockMatch when tested live on the Pioneer robot. Numbers indicate start positions and intersecting dashed lines indicate snapshot positions. 


\subsubsection{Analysis}

It is an unexpected result that BlockMatch should perform so well. The vector mapping stage assumes that correct correspondences have been established, and it is surprising that block matching should be able to deliver sufficiently correct correspondences, especially since the image features to be corresponded have undergone significant distortions due to scaling, perspective, occlusion, and illumination changes. Computer vision researchers have invented a multitude of image matching strategies dedicated towards robustly handling one or all of these distortions (see [66] for a review of recent approaches). All of this activity would not have taken place if block matching-an easily-understood, and well known technique-had been able to satisfy the needs of these researchers. Yet it does seem to satisfy the needs for visual homing, at least within the framework described here.

The approach we now take is to try and understand BlockMatch's success with the goal of replacing it by even simpler methods.

\section{The Democracy Effect}

An examination of the correspondence vectors generated by BlockMatch reveals that not all image features are corresponded correctly. Figure 8.8 shows a snapshot and current image pair, along with the correspondence vectors computed by BlockMatch between them. The current image was taken from a position to the left of the snapshot position. The image position in the current view corresponding to the correct home direction is $(52,23)$. Thus, at this position we expect to find the FOC, which means that the FOE should be half of the image width away at $(155,23)$. Contraction can be observed around the FOC and expansion around the FOE. However, the number of clearly incorrect vectors increases with displacement from the goal. Figure 8.9 shows flow fields for positions further and further to the left of the goal. Despite the increasingly poor quality of these flow fields, the computed home vectors for all of these positions are still approximately correct. This can be verified by examining the home vectors generated for these positions in figure 8.1(c).

The method is able to cope with incorrect correspondences as long as the overall number of correspondence vectors remains high. To test this hypothesis the step size, $m=m_{x}=m_{y}$, was varied in increasing powers of 2 . We increased step size in powers of 2 so that each set of correspondence vectors is a subset of the previous set of vectors-this would not be the case for a linear increase in step size. The total number of origin points decreases quadratically with increasing step size.

Figure 8.10 (a) shows a clear increase in $A A E_{*}$ (original) for increasing step size. This effect stems from the uncorrelated mismatches assumption, described in section 5.4.1. As 


\section{Snapshot \\ Image $(6,4)$}

Current

Image $(5,4)$

Correspondence

Vectors
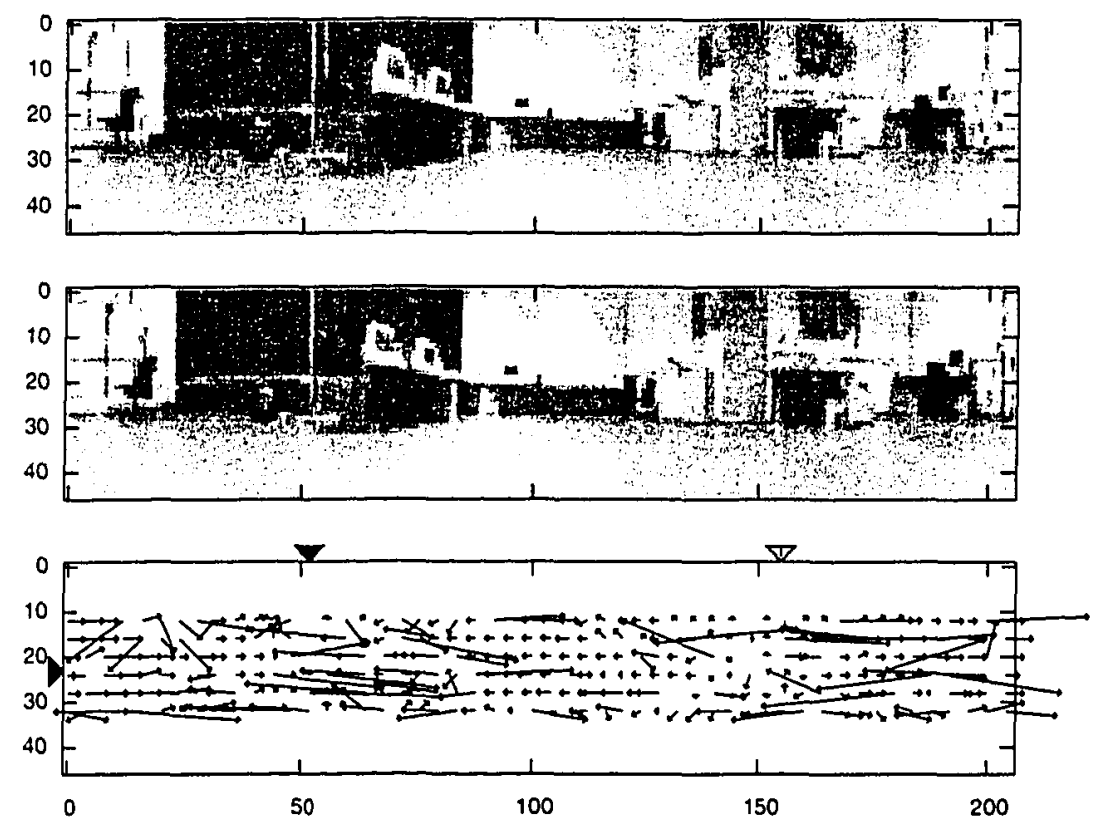

Figure 8.8: Example flow field for snapshot position $(6,4)$ and current position $(5,4)$. The correct home vector is directed towards the right, meaning that the correct FOC should be around $(52,23)$ while the correct FOE should be near $(155,23)$. These positions are indicated in (c) by triangles along the plot boundary. The horizontal position of the FOC is indicated by the filled triangle, while the FOE is indicated by the open triangle.

step size increases, the number of origin points decreases. With a small number of origin points, the incorrect correspondences will cancel each other out less effectively: Further, there may be a change in the ratio of correct to incorrect correspondences if we sample the image less densely. Thus, as we include more origin points, each 'voting' for home vectors, the final summed home vector becomes more correct. We refer to this as the democracy effect.

\section{Frequency content}

It was initially assumed that it was the high-frequency structure of image patches which allowed blocks to be distinguished from each other and correctly matched. However, this turns out not to be the case. If the high-frequency structure of local image patches was indeed the basis for BlockMatch's success then we should see performance decline as this 


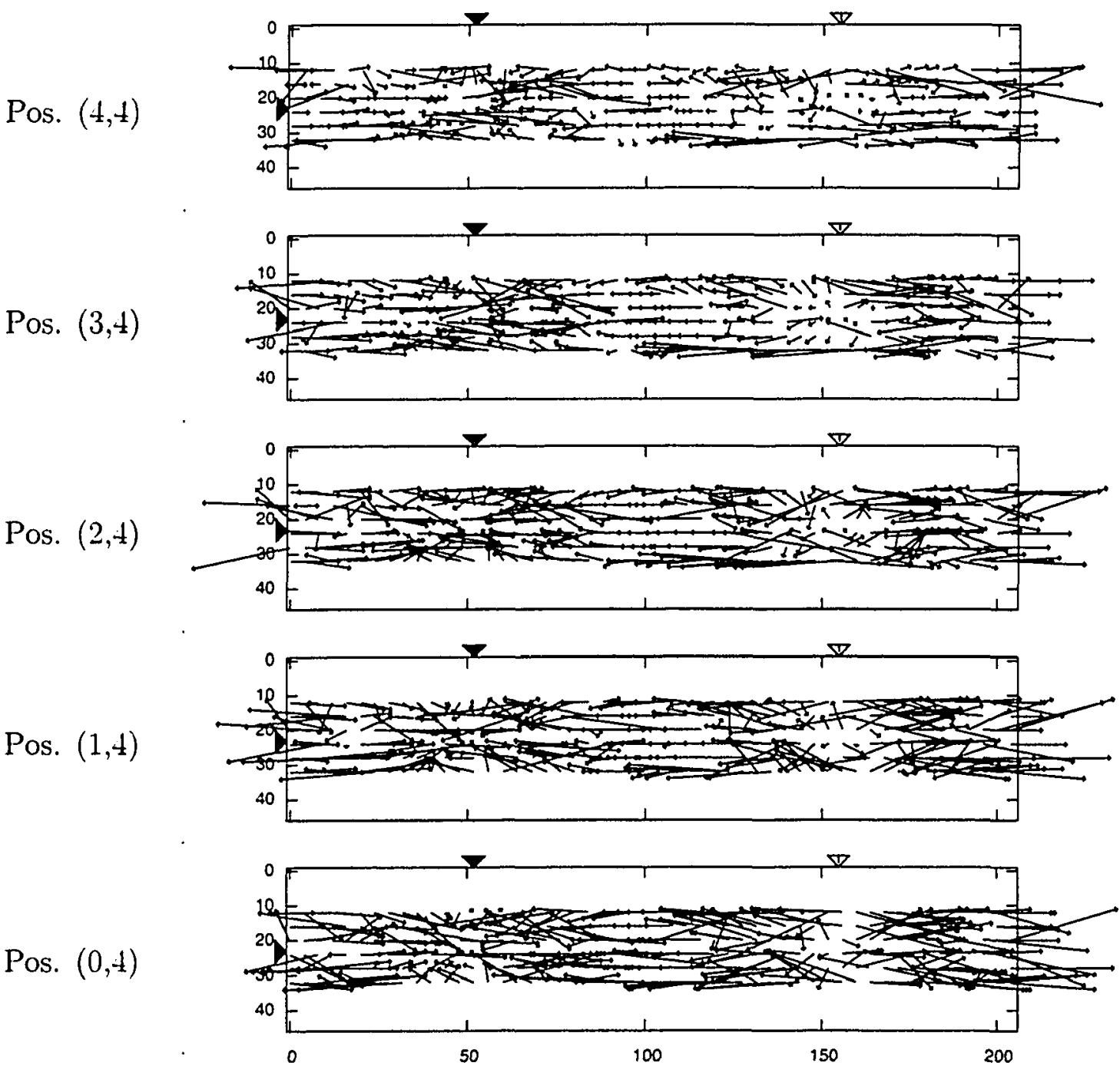

Figure 8.9: Flow fields for snapshot position $(6,4)$ and current positions from $(4,4)$ to $(0,4)$. Ideal positions of the FOC and FOE are indicated as in figure 8.8. 


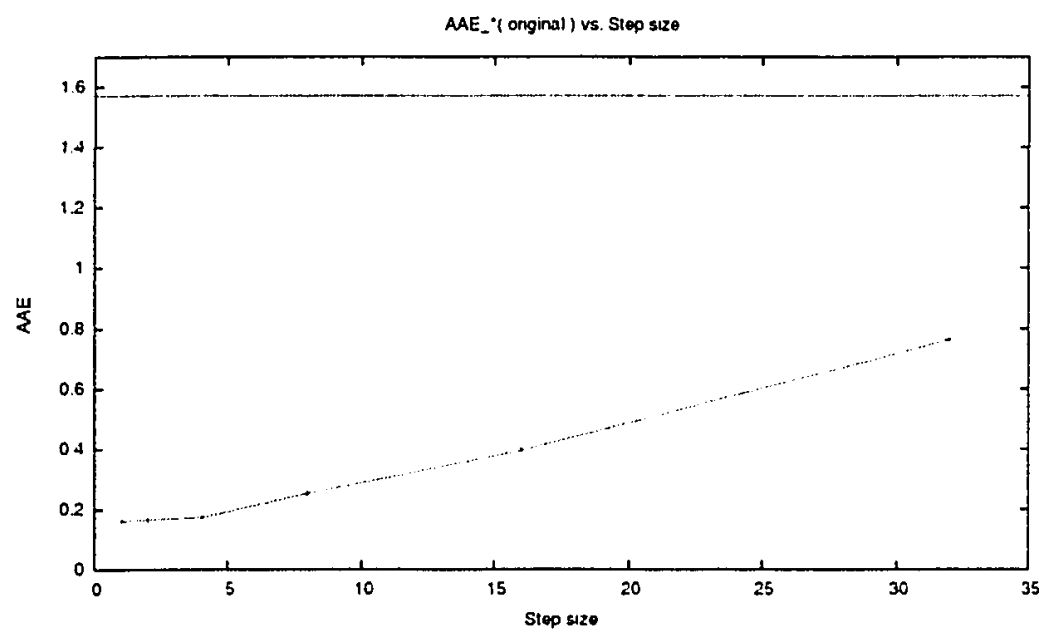

(a)

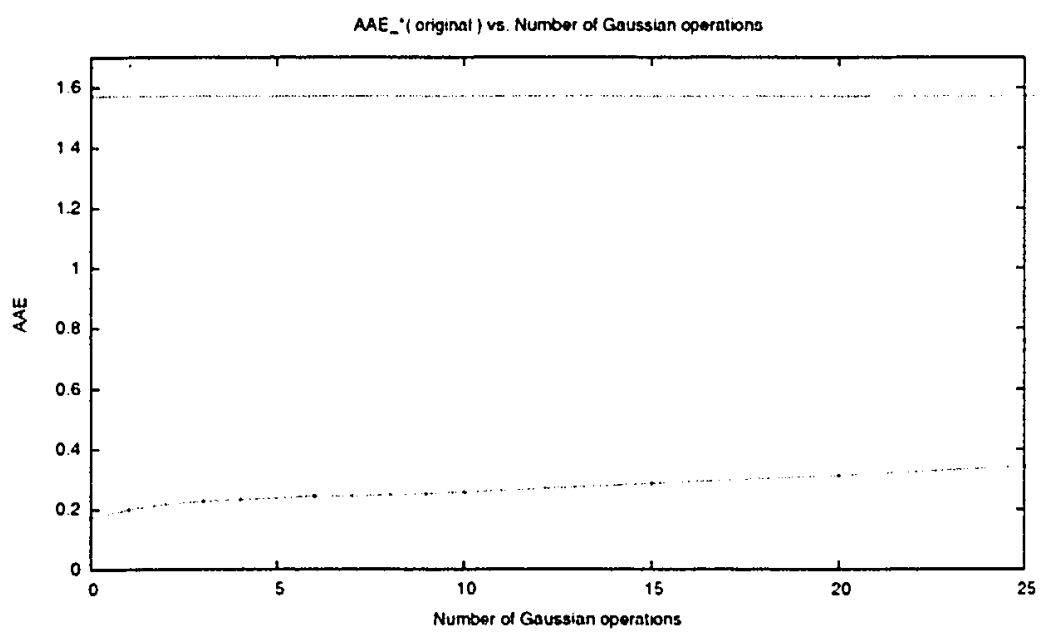

(b)

Figure 8.10: Plots of angular error vs. parameters. A dashed line is shown at $\pi / 2$. 
high-frequency structure is filtered out. The following experiment reveals only a slight decline in performance when the high-frequency structure is filtered out.

BlockMatch was tested with parameters as previously clescribed, with the exception that the input was filtered by repeated application of the Gaussian kernel (see section 6.5.3). Repeated convolution of a signal by a Gaussian kernel is equivalent to convolution of that signal with a wider Gaussian kernel. So by repeatedly applying a Gaussian filter, more and more of the high-frequency content is filtered out of the signal. Figure 8.10 (b) shows the value of $A A E_{*}$ (original) for an increasing number of filter applications. The angular error increases somewhat with more filtering, but even with very heavy filtering it remains far below $\frac{\pi}{2}$ (recall from section 5.3.1 that an angular error of $\frac{\pi}{2}$ has a special meaning for homing). To give a visual impression, figure 8.11 shows image $(6,4)$ from image collection original and the effects of 5 and 25 filtering operations on this image.

Image $(6,4)$

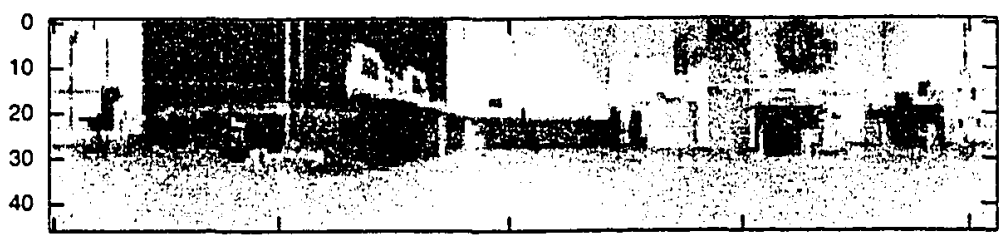

Filter $\times 5$
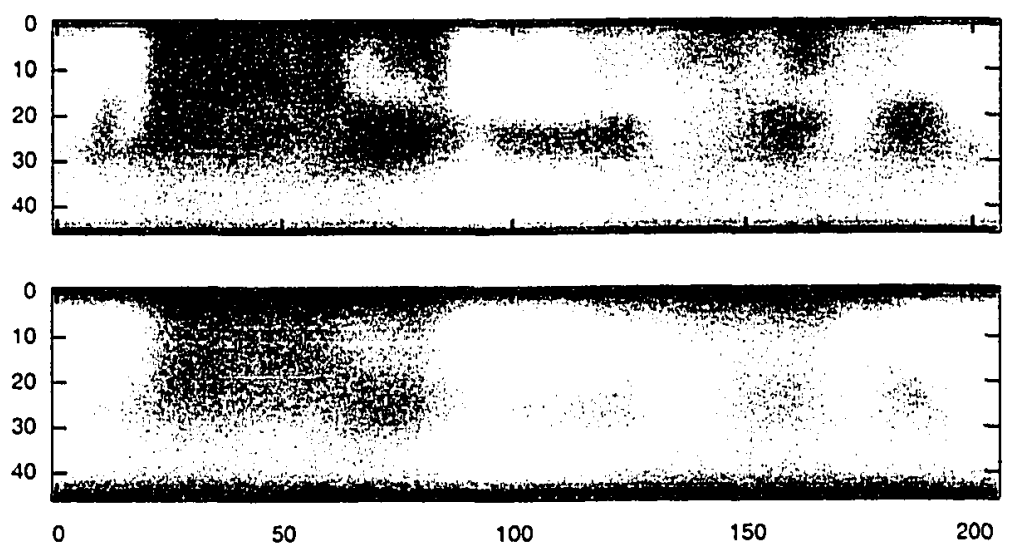

Filter $\times 25$

Figure 8.11: The effects of increased Gaussian filtering on image $(6,4)$ from image collection original.

The experiment shows that it must be the low-frequency image components which BlockMatch matches between images. By definition, low-frequency components are those that vary slowly across the image. This insight leads us directly along the path to minimal image descriptors. 


\subsection{IntMatch and GradMatch}

We conclude from the experiment above that BlockMatch actually pairs blocks whose lowfrequency components match. The simplest low-frequency component of a block is just its overall intensity. The next simplest low-frequency component is the change in overall intensity, which is a two-dimensional quantity given by the gradient of the intensity. We implemented two new homing methods based on matching the intensity and matching the gradient of intensity. We will refer to these new methods as IntMatch and GradMatch, respectively. IntMatch is essentially the same as BlockMatch with a block radius of zero. That is, to assess the quality of a potential match between $\mathbf{p}$ and $\mathbf{p}^{\prime}$ we do not compute the SSD but instead compute

$$
\left|S(\mathbf{p})-C\left(\mathbf{p}^{\prime}\right)\right|
$$

GradMatch, involves the additional step of computing the gradient of the image intensity. The image gradient was defined in chapter 6 as the vector of first derivatives of the image in the $x$ and $y$ directions. These derivatives can be approximated by convolving the image with the kernels mentioned 6 .

For GradMatch the gradient is computed for both the snapshot and current images. The quality of a match is judged by the quantity

$$
\left\|\nabla S(\mathbf{p})-\nabla C\left(\mathbf{p}^{\prime}\right)\right\|
$$

While the entities matched between snapshot and current image differ for BlockMatch, IntMatch, and GradMatch, they all find correspondence vectors between images and then apply the same vector mapping and summation stages to arrive at a final home vector.

\subsubsection{Pre-Processing}

A repeated Gaussian filter was applied to images before inputting them to either IntMatch or GradMatch. One of the parameters searched over below controlled the number of applications of this filter. As for BlockMatch, the use of a Sobel filter to enhance edges was also made available to the parameter search.

\subsubsection{Optimizing Parameters}

The parameters tested for both IntMatch and GradMatch are as follows:

- Number of applications of Gaussian filter: $0,1,2, \underline{3}$, or 4 . 
- Use Sobel filter: true or false.

- Number of top and bottom 10 image rows to exclude: $\underline{\mathbf{0}}, 5$, or 10.

- Search radius, $q: 5,10, \underline{30}$, or 50 .

The best parameters found for IntMatch are given above in bold. The best parameters for GradMatch have been underlined. Other parameters, such as step size, were set as for BlockMatch.

\subsubsection{Results on Image Database}

\section{Image Collection original}

Figure 8.12 shows home vectors generated by IntMatch and GradMatch on image collection original. Both methods perform remarkably well. IntMatch performs noticeably better on snapshot position $(0,16)$. These excellent home vector fields provide initial support for the theory that matching these very simplistic image descriptors can suffice for visual homing.

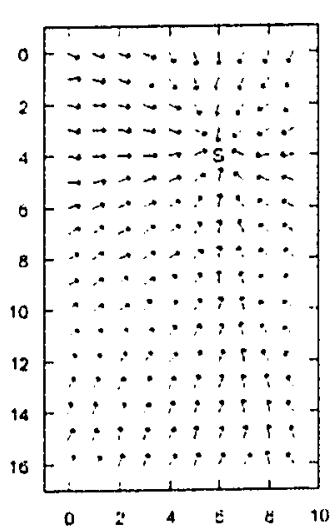

(a) IntMatch, $\mathbf{s}=(6,4)$ : $A . A E=0.207$, $R R=1.000$

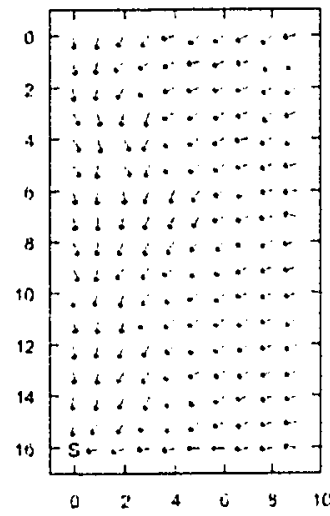

(b) IntMatch, $\mathbf{s}=(0,16):$ $A A E=0.322$, $R R=1.000$

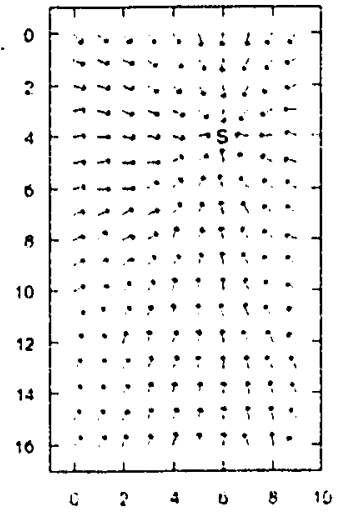

(c) GradMatch, $s=(6,4)$ : $A .4 E=0.159$, $R R=1.000$

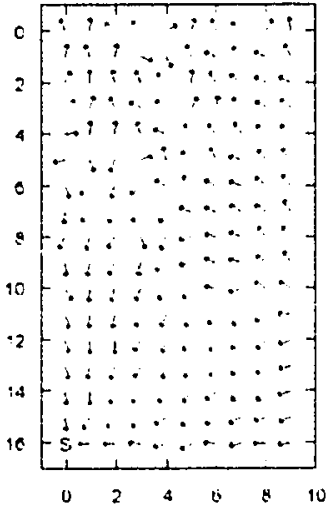

(d) GradMatch, $\mathrm{s}=(0,16)$ : $\triangle A E=1.095$, $R R=0.598$

Figure 8.12: Home vector fields for IntMatch $(a, b)$ and GradMatch $(c, d)$, for snapshot positions $(6,4)(a, c)$ and $(0,16)(b, d)$ in image collection original.

Figure 8.2 plots $A A E$ and $R R$ across snapshot positions. These plots show that the excellent quality of the home vector fields described above are representative. The plots for 
IntMatch in particular show no areas were performance is weak. The plots for GradMatch show a slight degradation in performance for the bottom portion of the capture grid.

\section{Image Collections hall1 and hall2}

The home vector fields for hall1 and hall2 are also quite good, although the performance for IntMatch is not quite as strong as GradMatch. Figure 8.13 shows these home vector fields for snapshot position $(3,12)$.

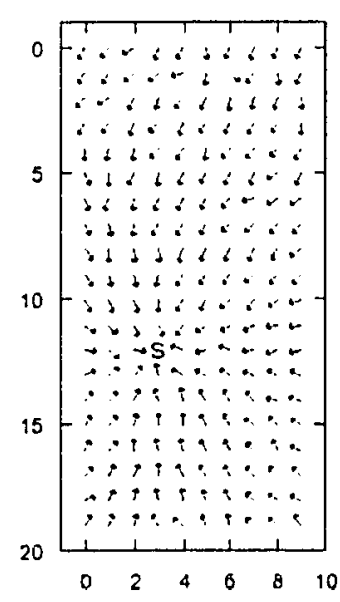

(a) IntMatch, hall1, $\mathbf{s}=(3,12)$ : $A . A E=0.270$, $R R=1.000$

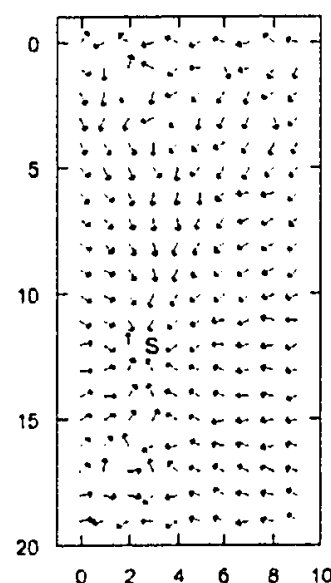

(b) IntMatch, hall2, $\mathrm{s}=(3,12):$ $A . A E=0.543$, $R R=0.864$

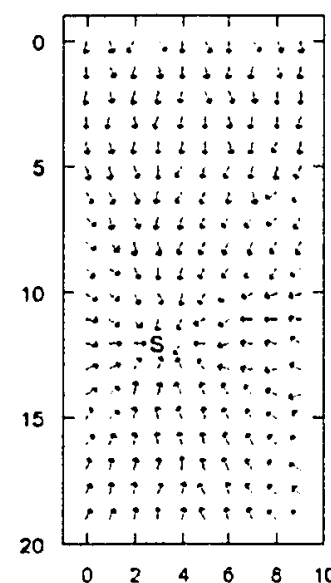

(c) GradMatch, hall1, $\mathrm{s}=(3,12)$ : $A A E=0.229$, $R R=1.000$

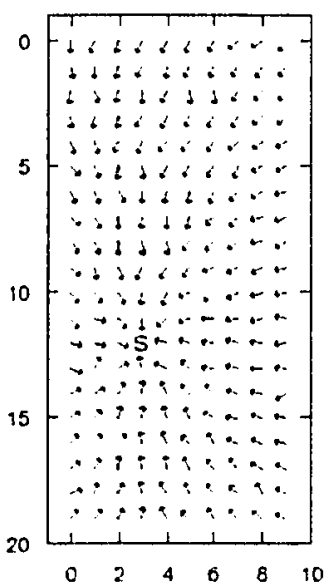

(d) GradMatch, hall2, $\mathrm{s}=(3,12)$ : $A A E=0.238$, $R R=1.000$

Figure 8.13: Home vector fields for IntMatch $(a, b)$ and GradMatch $(c, d)$, for snapshot position $(3,12)$ in image collections hall1 and hall2.

Figures 8.4 and 8.5 plots our performance metrics across snapshot positions for the two hall image collections. GradMatch stands out in these plots as exhibiting consistently low $A A E$ and high $R R$. IntMatch does not appear to perform as strongly, particularly in hall2. It is clear that neither method performs as strongly as BlockMatch.

\section{Cross-Collection Tests}

When snapshot and current images are talien from different image collections, the two simplified matching methods still perform quite impressively. Figure 8.6 presents the relevant 
plots. For these cross-collection tests GradMatch is again remarkable in exhibiting such consistently low $A A E$ and high $R R$. Only the combinations involving winlit appear to cause it much trouble. Meanwhile, IntMatch performs in a qualitatively similar fashion to BlockMatch.

\subsection{Comparative Results}

Statistical analyses were performed to compare the performance metrics for methods Warping, BlockMatch, IntMatch, and GradMatch. Table 8.2 presents the results on image collection original. For the $A A E$ metric, warping is outperformed by all other methods. For the $R R$ metric both IntMatch and GradMatch perform significantly better than Warping. Due to its perfect performance on image collection original, BlockMatch had to be excluded from Friedman's test for differences in $R R$.

Table 8.3 presents the results of the same tests for image collection hall1. In terms of $A A E$ all methods are significantly better than IntMatch and BlockMatch is better than Warping. The only difference for the $R R$ metric is that there is no longer a significant difference between IntMatch and Warping. The situation in image collection hall2 (see table 8.4) mirrors that described for hall1, with an added significant difference between BlockMatch and GradMatch in $A A E$.

Table 8.5 presents statistical analyses of the cross-collection tests. In terms of $A . A E$ all methods outperform Warping, and BlockMatch outperforms all other methods. Also, GradMatch outperforms IntMatch. The same situation is found for $R R$, with the exceptions that the differences between BlockMatch and GradMatch, and between Warping and IntMatch, are no longer significant.

In all of these tests BlockMatch remains unbeaten. GradMatch also does cuite well and is only ever outperformed by BlockMatch. IntMatch is outperformed by these two methods in various cases. However, it is outperformed by Warping only in the hall collections and only for the AAE metric.

While neither IntMatch or GradMatch outperform BlockMatch, they have both been shown to perform to a high standard. As GradMatch is never beaten by Warping it joins BlockMatch as a method that it is competitive with Warping. The case for IntMatch is less clear-cut but it remains a very interesting method.

It may be that BlockMatch should be viewed as a hybrid of IntMatch, GradMatch, and perhaps even high-frequency matching-the result in section 8.2.6 showed that high frequencies were not the primary component of block matching's success; but they may well 
Significance of difference in $A A E_{*}$ (original)

\begin{tabular}{|l|l|l|l|l|}
\hline & Warping & BlockMatch & IntMatch & GradMatch \\
\hline Warping & $\mathrm{X}$ & & & \\
\hline BlockMatch & $* * *$ & $\mathrm{X}$ & & \\
\hline IntMatch & $* * *$ & & $\mathrm{X}$ & \\
\hline GradMatch & $*$ & & & $\mathrm{X}$ \\
\hline
\end{tabular}

Significance of difference in $R R_{*}$ (original)

\begin{tabular}{|l|l|l|l|l|}
\hline & Warping & BlockMatch & IntMatch & GradMatch \\
\hline Warping & $\mathrm{X}$ & $\mathrm{NA}$ & & \\
\hline BlockMatch & $\mathrm{NA}$ & $\mathrm{NA}$ & $\mathrm{NA}$ & $\mathrm{NA}$ \\
\hline IntMatch & $* * *$ & $\mathrm{NA}$ & $\mathrm{X}$ & \\
\hline GradMatch & $* *$ & $\mathrm{NA}$ & & $\mathrm{X}$ \\
\hline
\end{tabular}

Table 8.2: Statistical significance of the difference in $A A E_{*}$ (original) and $R R_{*}$ (original) between homing methods. See table 4.1 for notation.

Significance of difference in $A A E_{*}$ (hall1)

\begin{tabular}{|l|l|l|l|l|}
\hline & Warping & BlockMatch & IntMatch & GradMatch \\
\hline Warping & $\mathrm{X}$ & & $* * *$ & \\
\hline BlockMatch & $*$ & $\mathrm{X}$ & $* * *$ & \\
\hline IntMatch & & & $\mathrm{X}$ & \\
\hline GradMatch & & & $* * *$ & $\mathrm{X}$ \\
\hline
\end{tabular}

Significance of difference in $R R_{*}$ (hall1)

\begin{tabular}{|l|l|l|l|l|}
\hline & Warping & BlockMatch & IntMatch & GradMatch \\
\hline Warping & $\mathrm{X}$ & & & \\
\hline BlockMatch & $*$ & $\mathrm{X}$ & $* *$ & \\
\hline IntMatch & & & $\mathrm{X}$ & \\
\hline GradMatch & & & $* *$ & $\mathrm{X}$ \\
\hline
\end{tabular}

Table 8.3: Statistical significance of the difference in $A A E_{*}$ (hall1) and $R R_{*}$ (hall1) between homing methods. See table 4.1 for notation. 
Significance of difference in $A A E_{*}$ (hal12)

\begin{tabular}{|l|l|l|l|l|}
\hline & Warping & BlockMatch & IntMatch & GradMatch \\
\hline Warping & $\mathrm{X}$ & & $* * *$ & \\
\hline BlockMatch & $* *$ & $\mathrm{X}$ & $* * *$ & $* *$ \\
\hline IntMatch & & & $\mathrm{X}$ & \\
\hline GradMatch & & & $* * *$ & $\mathrm{X}$ \\
\hline
\end{tabular}

Significance of difference in $R R_{*}($ hall2)

\begin{tabular}{|l|l|l|l|l|}
\hline & Warping & BlockMatch & IntMatch & GradMatch \\
\hline Warping & $\mathrm{X}$ & & & \\
\hline BlockMatch & $* * *$ & $\mathrm{X}$ & $* * *$ & \\
\hline IntMatch & & & $\mathrm{X}$ & \\
\hline GradMatch & & & $* * *$ & $\mathrm{X}$ \\
\hline
\end{tabular}

Table 8.4: Statistical significance of the difference in $A A E_{*}$ (hall2) and $R R_{*}$ (hall2) between homing methods. See table 4.1 for notation.

Significance of difference in $A . A E_{*}(*, *)$

\begin{tabular}{|l|l|l|l|l|}
\hline & Warping & BlockMatch & IntMatch & GradMatch \\
\hline Warping & $\mathrm{X}$ & & & \\
\hline BlockMatch & $* * *$ & $\mathrm{X}$ & $* * *$ & $*$ \\
\hline IntMatch & $* * *$ & & $\mathrm{X}$ & \\
\hline GradMatch & $* * *$ & & $*$ & $\mathrm{X}$ \\
\hline
\end{tabular}

Significance of difference in $R R_{*}(*, *)$

\begin{tabular}{|l|l|l|l|l|}
\hline & Warping & BlockMatch & IntMatch & GradMatch \\
\hline Warping & $\mathrm{X}$ & & & \\
\hline BlockMatch & $* * *$ & $\mathrm{X}$ & $* * *$ & \\
\hline IntMatch & & & $\mathrm{X}$ & \\
\hline GradMatch & $*$ & & $* *$ & $\mathrm{X}$ \\
\hline
\end{tabular}

Table 8.5: Statistical significance of the difference in $A A E_{*}(*, *)$ and $R R_{*}(*, *)$ between homing methods for all pairings of image collections from the office environment. See table 4.1 for notation. 
be useful for certain kinds of images. Thus, in certain environments BlockMatch would employ intensity matching, while in others gradient matching or high-frequency matching might be preferred.

\subsection{Complexity and Biological Plausibility}

\subsubsection{BlockMatch}

While BlockMatch is conceptually simple, it requires an expensive search for each correspondence. On a $\mathrm{PC}$ with a Pentium $42 \mathrm{GHz}$ processor it takes approximately $327 \mathrm{~ms}$ to compute a home vector. In terms of pixel-to-pixel comparisons, each correspondence search requires a number of comparisons equal to

$$
\mathcal{C}_{\text {scarch }}=(2 r+1)^{2}(2 q+1)^{2}
$$

The total number of operations required to compute a home vector is

$$
\mathcal{C}_{\text {BIockMatch }}=\mathcal{C}_{\text {scarch }} n_{x} n_{y}
$$

For the parameters selected in section 8.2 .2 the quantity above comes to almost 20 million. Using the rough assumptions of one neuron for each pixel-to-pixel comparison, it remains clearly impossible for all operations to be carried out in parallel in the brain of an insect with only about one million neurons.

\subsubsection{IntMatch and GradMatch}

The computational complexities for IntMatch and GradMatch are clearly reduced from BlockMatch. These methods take an average of $86.5 \mathrm{~ms}$ and $224.5 \mathrm{~ms}$, respectively; to compute a home vector on the same computer as used above.

Where $f=1$ for IntMatch, and $f=2$ for GradMatch, the number of operations required to compute a home vector is

$$
\mathcal{C}_{\text {IntMatch,GradMatch }}=f(2 q+1)^{2} n_{x} n_{y}
$$

This also does not include the cost of pre-processing. For the parameters selected for IntMatch, this quantity comes to approximately 260,000. For GradMatch the cost is about 4.4 million. The search radius selected for IntMatch was 10 as opposed to 30 for GradMatch. The cost of BlockMatch with a search radius of 10 is approximately 520,000 . 
Clearly these methods are more biologically-plausible for insects than BlockMatch. However, it still seems quite unlikely that 260,000 of the 850,000 neurons in a honeybee's brain would be dedicated to this one task of visual homing. Indeed, if this were the case then the neural apparatus implementing the correspondence search process would be quite prevalent in neurophysiological studies. While such studies do reveal extensive horizontal interconnections between columns of the retinotopic lattice which characterizes the optic lobes of bee brains $[42,86]$, it seems unlikely that the dense interconnectivity required for correspondence search would have gone unnoticed.

\subsection{Conclusions}

This chapter investigated the question of finding minimal image descriptors sufficient for competitive homing performance. The question was motivated by the method SIMatch, presented in the previous chapter, which was found to be (nearly) competitive with Warping but only at the expense of high computational complexity. We began by testing BlockMatch which extracts a much lighter description of a local image region than SIMatch. BlockMatch was found to be more than competitive with Warping. Indeed, based on the experiments conducted here it can be concluded that BlockMatch represents a new state of the art in visual homing.

The analysis of BlockMatch revealed that just the low-frequency components of the image were sufficient for homing. Further, it was found that increasing the number of matches only improved performance. This finding stands in stark contrast to the usual approach within computer vision whereby features are selected with great care and retained only if they meet some minimum standard for reliability (e.g. [66]).

The question of finding a minimal image descriptor was pursued directly by testing the two most minimal descriptors conceivable: image intensity and gradient. Quite surprisingly, these descriptors were indeed sufficient. The method based on image intensity, IntMatch performed quite well in some environments, such as in image collection original. However, it performed less well on the hall collections. Nevertheless, it maintained a high level of performance in all environments - with regards to the $R R$ metric there were no statistically. significant differences with Warping. The method based on image gradients, GradMatch performed competitively (as good or better) to Warping in all environments.

In the meantime, while the goal of finding minimal image descriptors has been reached, we are still left with methods that correspond these descriptors through a search process. Yet, if the descriptors themselves are so lightweight, and if bad correspondences can be 
tolerated, might there not be a way to find correspondences that avoids search? This question is the subject of the next chapter. 


\section{Chapter 9}

\section{Differential Methods}

\subsection{Introduction}

The previous chapter proposed three variants on the concept of establishing correspondences by searching for the best matches between image descriptors. The methods IntMatch and GradMatch were found to perform quite well with very minimal image descriptors. However, these methods still require an intensive local search process for every correspondence. This chapter investigates whether differential techniques can be used to establish correspondences without search. Differential techniques assume that image brightness is conserved between the snapshot and current images. They also assume that all feature displacements are relatively small. Despite these two strong assumptions it turns out that differential techniques are very useful for visual homing. They also permit the search process to be avoided and are easily implementable within a retinotopic matrix. Thus, only in this chapter do we arrive at methods that fulfill all of the criteria stated in chapter 1.

Two differential methods are develuped in this chapter. These methods are based on IntMatch and GradMatch in that they correspond pixel intensity; and the gradient of intensity, respectively. The assumptions underpinning these methods will be carefully considered. After discussing the results on these methods we will again delve into the question of how the methods' assumptions are upheld.

Note that this chapter describes joint work by Vardy and Möller [98]. 


\subsection{Differential Techniques}

The differential equivalents of IntMatch and GradMatch are presented below. The method based on IntMatch employs first-order derivatives to estimate the direction of feature translation. Thus, we refer to this method as FirstOrder. The method based on GradMatch employs second-order derivatives and is therefore referred to as Second0rder. Both methods rely on an initial assumption that image brightness is conserved. These methods also employ a Taylor approximation to determine the direction of feature translation. Such an approximation is valid only for small feature translations. Some justification for the use of the Taylor approximation is provided below.

In the case of Firstorder the feature which experiences translation is just a pixel with a particular intensity: For Second0rder the feature which experiences translation is a vector-the gradient of intensity. Finding the actual translation of a feature from the snapshot image to the current image is equivalent to finding a correspondence vector.

The length of correspondence vectors estimated by the differential methods was thought to be relatively inaccurate. Therefore, a new approximate vector mapping technique was developed. The exact vector mapping technique of chapter 5 requires that both the direction and distance of feature translation be known. The new vector mapping technique follows the presentation of FirstOrder and SecondOrder and the discussion of their assumptions.

\subsubsection{FirstOrder}

As usual, $S$ and $C$ represent the snapshot and current images, respectively. $S(x, y)$ and $C(x, y)$ denote the intensity of these images at coordinates $(x, y)$. The assumption that the intensity is unchanged under an image translation $(u, v)$ between the two images is expressed by

$$
C(x+u, y+v)=S(x, y) .
$$

Following the standard approach to differential techniques reviewed by Barron et al. [2], we apply a first-order Taylor series expansion on the left-hand side of this equation to obtain

$$
C(x, y)+\left[C_{x}(x, y), C_{y}(x, y)\right]\left(\begin{array}{l}
u \\
v
\end{array}\right)=S(x, y),
$$

with $C_{x}$ and $C_{y}$ denoting partial derivatives with respect to $x$ and $y$. Note that this Taylor series approximation is valid only for small translations.

With arguments $x, y$ omitted, we can write

$$
\left(C_{x}, C_{y}\right)\left(\begin{array}{l}
u \\
v
\end{array}\right)=S-C
$$


Here we have two unknowns, $u$ and $v$, constrained by only one equation. Thus, there are infinitely many solutions. Therefore we add the following constraint

$$
\left(\begin{array}{l}
u \\
v
\end{array}\right)=k\left(\begin{array}{l}
C_{x} \\
C_{y}
\end{array}\right)
$$

This constraint implies that the unknown correspondence vector $(u v)^{T}$ is aligned with the image gradient. It is a well-known problem that purely local differential techniques can only estimate that component of the correspondence vector which is parallel to the image gradient. This is known as the aperture problem [96] (page 192). We simply accept that the vector $(u v)^{T}$ will only be that component of the true correspondence vector which is parallel to the gradient.

We can solve for the constant $k$ by combining equations (9.3) and (9.4)

$$
k=\frac{S-C}{\left\|\left(\begin{array}{l}
C_{x} \\
C_{y}
\end{array}\right)\right\|^{2}}
$$

Incorporating this into $(9.4)$ yields

$$
\left(\begin{array}{l}
u \\
v
\end{array}\right)=(S-C) \frac{\left(\begin{array}{l}
C_{x} \\
C_{y}
\end{array}\right)}{\left\|\left(\begin{array}{l}
C_{x} \\
C_{y}
\end{array}\right)\right\|^{2}}
$$

It proved to be sufficient to ignore the length of the translation vector: therefore we use

$$
\left(\begin{array}{l}
u \\
v
\end{array}\right)=\operatorname{sgn}(S-C) \frac{\left(\begin{array}{l}
C_{x} \\
C_{y}
\end{array}\right)}{\left\|\left(\begin{array}{l}
C_{x} \\
C_{y}
\end{array}\right)\right\|}
$$

(for a zero gradient vector, the translation vector is set to zero). The function $\operatorname{sgn}(\cdot)$ delivers the sign of its argument:

$$
\operatorname{sgn}(x)=\left\{\begin{array}{ll}
-1 ; & x<0 \\
0 ; & x=0 \\
1 ; & x>0
\end{array} .\right.
$$

For the discrete approximation of the first-order derivatives, any first-order edge filter kernel can be applied to the image. Here we use the same kernels as were employed previously in chapters 6 and 8: $\left(\begin{array}{lll}1 & 0 & -1)\end{array}\right)$ and $\left(\begin{array}{lll}1 & 0 & -1\end{array}\right)^{T}$. 


\subsubsection{SecondOrder}

The second-order method rests on the assumption that the intensity gradient is preserved under translations [2], which can be expressed as

$$
\begin{aligned}
& C_{x}(x+u, y+v)=S_{x}(x, y) \\
& C_{y}(x+u, y+v)=S_{y}(x, y)
\end{aligned}
$$

From a Taylor expansion on the left-hand side and approximation for small translations we get

$$
\begin{aligned}
& C_{x}+\left(C_{x x}, C_{x y}\right)\left(\begin{array}{l}
u \\
v
\end{array}\right)=S_{x} \\
& C_{y}+\left(C_{y x}, C_{y y}\right)\left(\begin{array}{l}
u \\
v
\end{array}\right)=S_{y},
\end{aligned}
$$

(with $C_{x x}, C_{x y}, C_{y x}$, and $C_{y y}$ denoting second-order partial derivatives) which can be summarized in a single equation [2]:

$$
\left(\begin{array}{ll}
C_{x x} & C_{x y} \\
C_{y x} & C_{y y}
\end{array}\right)\left(\begin{array}{l}
u \\
v
\end{array}\right)=\left(\begin{array}{l}
D_{x} \\
D_{y}
\end{array}\right) .
$$

Here we use $D=S-C$. Note that $C_{x y}=C_{y x}$ holds for continuous derivatives. For the non-singular case, we obtain the solution

$$
\begin{aligned}
u & =\frac{D_{x} C_{y y}-D_{y} C_{x y}}{C_{x x} C_{y y}-C_{y x} C_{x y}} \\
v & =\frac{-D_{x} C_{y x}+D_{y} C_{x x}}{C_{x x} C_{y y}-C_{y x} C_{x y}} .
\end{aligned}
$$

For the application in visual homing, we found that it is sufficient to only consider the sign of the components:

$$
\begin{aligned}
& u=\operatorname{sgn}\left(D_{x} C_{y y}-D_{y} C_{x y}\right) \operatorname{sgn}\left(C_{x x} C_{y y}-C_{y x} C_{x y}\right) \\
& v=\operatorname{sgn}\left(-D_{x} C_{y x}+D_{y} C_{x x}\right) \operatorname{sgn}\left(C_{x x} C_{y y}-C_{y x} C_{x y}\right)
\end{aligned}
$$

With these equations, we obtain a zero vector in the singular case. Second-order derivatives are approximated by two subsequent convolutions of the image with the filter kernels described above. 


\subsection{Assumptions}

For First0rder, it is assumed that the pixel intensity is conserved between the snapshot and current images. For SecondOrder, it is assumed that the gradient of intensity is conserved (which implicitly assumes that intensity is also conserved). These assumptions are bound to be unfulfilled in many real situations. However, it is one of the basic assumptions of visual homing that something is conserved between images. For SIMatch it was assumed to be the local pattern of edges. For BlockMatch it was the local pattern of pixels. For IntMatch and GradMatch the conserved entities were the same as for FirstOrder and SecondOrder. Thus, while the differential methods make such strong assumptions, so does every other method based on matching features between images. Further, the excellent performance of IntMatch and GradMatch from the last chapter lend support to the particular conservation assumptions of the differential methods.

More troubling, perhaps, is the assumption of small feature translations. Both methods employ a Taylor series approximation that is valid only for small shifts of the intensity, or gradient of intensity, between images. This assumption is clearly not fulfilled if the two images are taken from disparate positions in the environment. Does this constrain the differential methods to be effective only in the immediate vicinity of the goal? Perhaps not, as there are two factors that justify this assumption.

The first factor becomes apparent when we consider the formula for the error of a Taylor approximation. The error for a first order Taylor approximation is defined as follows [93]:

$$
\left|\frac{1}{2} f^{\prime \prime}(z)(x-c)^{2}\right|
$$

where $f$ is the function approximated and the goal is to approximate $f(x)$. The value of $f$, and its first derivative are known at $c$. The quantity $z$ is an unknown bound only to lie somewhere in the range $[x, c]$. The term $(x-c)$ is the shift between the position where $f$ is known $(c)$, and where the approximation is clesired $(x)$. The fact that error is proportional to $(x-c)^{2}$ implies that the size of shift must be kept small. However, for any given shift the error will depend on the size of $f^{\prime \prime}(z)$. A function with small clerivatives will therefore exhibit lower error. By definition, a lorv-frequency function has small derivatives. This analysis holds if we consider not a one-dimensional function but a two-dimensional image.

Thus, errors in the Taylor approximation are reduced for images with significant energy in the low-frequency band. It was already demonstrated in the previous chapter that sufficient low-frequency components exist in images from the Bielefeld database for methods like IntMatch and GradMatch to succeed. Further, it is known that natural images exhibit a 
strong portion of low frequencies $[90,97]$. This gives us reason to believe that the differential methods will be successful.

The second factor relates to the geometry of visual homing. It was shown in chapter 2 that image regions near the FOC and FOE will exhibit very little flow. In these regions the requirement of small shifts will be satisfied. On the other hand, image regions far from the FOC and FOE will exhibit the largest flows. The region of small shifts near the foci will ideally generate approximately correct correspondence vectors. From these approximately correct correspondence vectors we will obtain approximately correct home vectors all directed in a consistent direction. The image region of large shifts arvar from the foci will ideally generate uncorrelated correspondence vectors and therefore uncorrelated home vectors. Thus, the uncorrelated home vectors will cancel themselves out and leave an approximately correct home vector. This is the assumption of uncorrelated mismatches from section 5.4.1.

As usual it will be an empirical question whether the assumptions and conditions discussed above will be sufficiently satisfied to allow the differential methods to perform competitively.

\subsection{Approximate Vector Mapping}

As FirstOrder and SecondOrder only estimate the direction, and not the distance, of correspondence it is not possible to use the vector mapping method of chapter 5 . Therefore this section develops a new approximate vector mapping technique.

In the robot coordinate system $\mathrm{x}_{R}, \mathrm{y}_{R}$, the individual home vector $\mathrm{v}_{h}$ can be expressed as the sum of the vector from $\mathrm{c}$ to $l$ and the vector from 1 to $\mathrm{s}$ :

$$
\mathrm{v}_{h}=d_{\mathbf{c}}\left(\begin{array}{c}
\cos \left(\theta_{x}+\delta_{x}\right) \\
\sin \left(\theta_{x}+\delta_{x}\right)
\end{array}\right)-d_{\mathbf{s}}\left(\begin{array}{c}
\cos \theta_{x} \\
\sin \theta_{x}
\end{array}\right)
$$

In the approximated vector mapping technique, the individual home vector is expressed in coordinates $(\rho, \tau)$ with respect to a coordinate system $\left(\mathbf{v}_{r}, \mathbf{v}_{t}\right)$ which is attached to the horizontal angle where the landmark $I$ appears in the snapshot (see figure 9.1):

$$
\mathbf{v}_{h}=\rho \mathbf{v}_{r}+\tau \mathbf{v}_{t}
$$

With the radial and tangential coordinate vector

$$
\mathbf{v}_{r}=\left(\begin{array}{c}
\cos \theta_{x} \\
\sin \theta_{x}
\end{array}\right), \quad \mathbf{v}_{t}=\left(\begin{array}{c}
\cos \left(\theta_{x}+\frac{\pi}{2}\right) \\
\sin \left(\theta_{x}+\frac{\pi}{2}\right)
\end{array}\right)=\left(\begin{array}{c}
-\sin \theta_{x} \\
\cos \theta_{x}
\end{array}\right)
$$




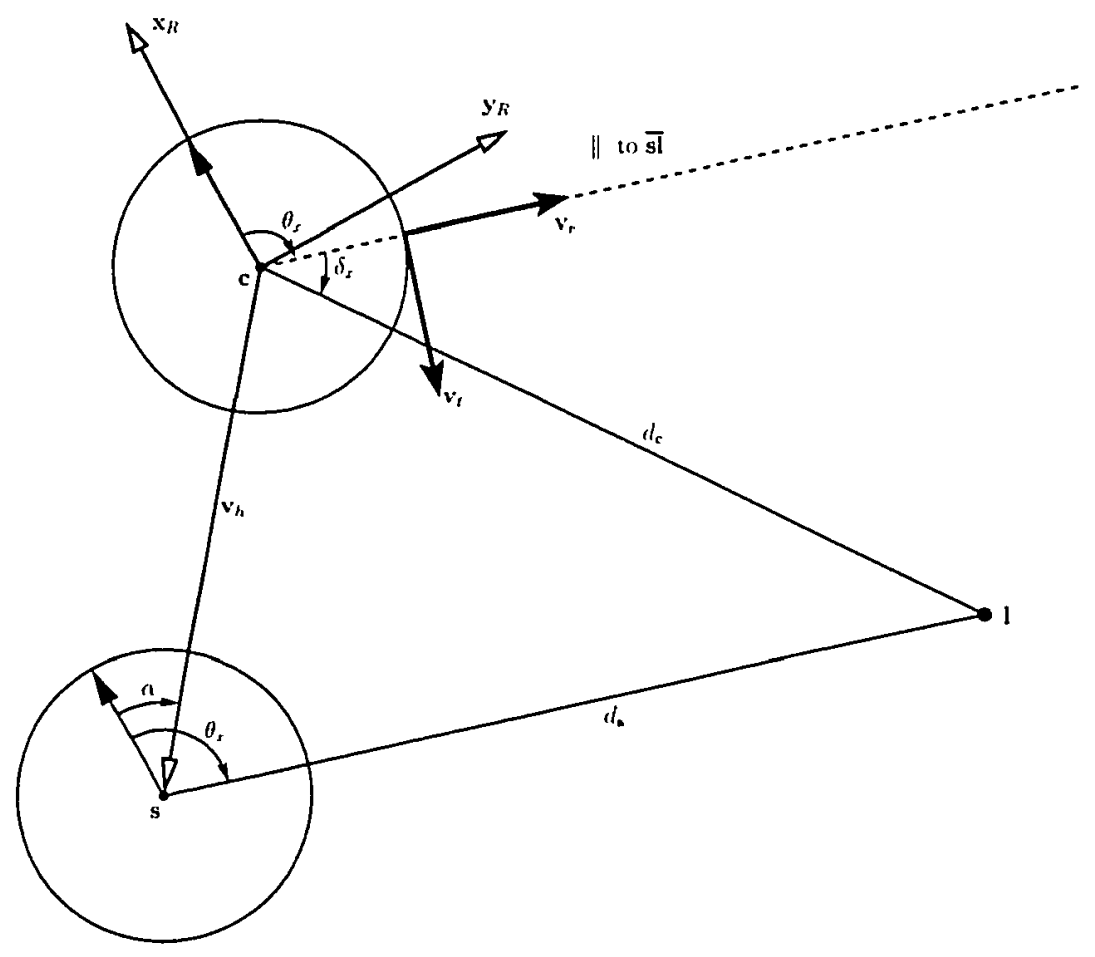

Figure 9.1: Illustration of the robot coordinate system and the vectors involved in the approximate vector mapping technique.

we obtain the following equation for the first vector component:

$$
\rho \cos \theta_{x}-\tau \sin \theta_{x}=d_{\mathrm{c}} \cos \left(\theta_{x}+\delta_{x}\right)-d_{\mathrm{s}} \cos \theta_{x} .
$$

After applying an addition theorem to the right-hand side and rearranging terms we arrive at

$$
\rho \cos \theta_{x}-\tau \sin \theta_{x}=\left(d_{\mathbf{c}} \cos \delta_{x}-d_{\mathbf{s}}\right) \cos \theta_{x}-d_{\mathbf{c}} \sin \delta_{x} \sin \theta_{x}
$$

which, by comparing the coefficients, gives

$$
\begin{aligned}
& \rho=d_{\mathrm{c}} \cos \delta_{x}-d_{\mathrm{s}} \\
& \tau=d_{\mathrm{c}} \sin \delta_{x}
\end{aligned}
$$

We are only interested in preserving the ratio of $\rho$ and $\tau$. By factoring out $d_{\mathbf{c}}$ and and approximating $\cos \delta_{x} \approx 1$ and $\sin \delta_{x} \approx \delta_{x}$ for small $\delta_{x}$, we obtain

$$
\begin{aligned}
\rho^{\prime} & =1-\frac{d_{\mathbf{s}}}{d_{\mathbf{c}}} \\
\tau^{\prime} & =\delta_{x}
\end{aligned}
$$


Recall from chapter 5 that the ratio $\frac{d_{\mathrm{s}}}{d_{\mathrm{c}}}$ can be obtained by considering the vertical component of correspondence, $\delta_{y}$. This allowed us to obtain equation (5.8), which is repeated below

$$
\frac{d_{\mathbf{s}}}{d_{\mathbf{c}}}=\frac{\tan \left(\theta_{y}+\delta_{y}\right)}{\tan \theta_{y}}
$$

we can now continue for $\rho^{\prime}$ with

$$
\rho^{\prime}=1-\frac{\tan \left(\theta_{y}+\delta_{y}\right)}{\tan \theta_{y}}
$$

For small vertical translations $\delta_{y}$, we can approximate $\tan \left(\theta_{y}+\delta_{y}\right)$ by a Taylor expansion up to first-order terms:

$$
\rho^{\prime}=1-\frac{\tan \theta_{y}+\delta_{y} \tan ^{\prime} \theta_{y}}{\tan \theta_{y}}=\frac{-\delta_{y}}{\tan \theta_{y} \cos ^{2} \theta_{y}}=\frac{-\delta_{y}}{\sin \theta_{y} \cos \theta_{y}} .
$$

For vertical landmark angles $\theta_{y}$ which stay close the horizon, we obtain the following approximation:

$$
\rho^{\prime}=-\frac{\delta_{y}}{\theta_{y}}
$$

Instead of using the value of $\theta_{y}$, we observed that only considering its sign leads to better homing performance, which gives

$$
\begin{aligned}
\rho^{\prime} & =-\operatorname{sgn}\left(\theta_{y}\right) \delta_{y} \\
\tau^{\prime} & =\delta_{x} .
\end{aligned}
$$

Finally, the individual home vector is normalized to unit length:

$$
\begin{aligned}
\rho^{\prime \prime} & =\frac{-\operatorname{sgn}\left(\theta_{y}\right) \delta_{y}}{\sqrt{\delta_{x}^{2}+\delta_{y}^{2}}} \\
\tau^{\prime \prime} & =\frac{\delta_{x}}{\sqrt{\delta_{x}^{2}+\delta_{y}^{2}}}
\end{aligned}
$$

A slight gain in performance is obtained for Firstorder if the home vector is subsequently multiplied by the length of the gradient $\left\|\left(C_{x} C_{y}\right)^{T}\right\|$. This is because the flow field can best be approximated by differential techniques where the image gradient is strongest (see [96], chapter 8). 


\subsection{Pre-Processing}

As demonstrated in the previous chapter, a matching of low-frequency components is sufficient for successful homing. For the differential methods in particular, high-frequency components may degrade performance. Therefore a Gaussian filter is applied to the input images. The number of applications of this filter is controlled by a parameter, optimized below. The number of applications of a stable Gaussian filter, as described in section 6.5.3 is also controlled by a parameter. The advantage of the stable filter is that it does not shrink the effective size of the image ${ }^{1}$. We prefer to keep the image at its maximum size to take advantage of the democracy effect, described in the last chapter. Meanwhile, there might also be benefits to cutting out regions of the floor and ceiling which are relatively indistinct. Therefore, the parameter search includes an additional parameter to remove rows from the top and bottom of the inage.

\subsection{Optimizing Parameters}

The parameters tested for both Firstorder and Second0rder are as follows:

- Number of applications of Gaussian filter: $\underline{0}, \mathbf{1}, 2,3,4$, or 5 .

- Number of applications of Gaussian filter (stable): $0,1,2,3,4$, or $\underline{5}$.

- Number of top and bottom image rows to exclude: $\underline{0}, 5$, or 10.

The best parameters found for Firstorder are given above in bold. The best parameters for Second0rder have been underlined. As these metlods are computationally cheap, we opt to compute correspondence vectors at all image positions, as opposed to a subset of image positions as was done for the matching methods. This again is intended to take advantage of the democracy effect.

\subsection{Results on Image Database}

\section{Image Collection original}

Figure 9.2 shows the home vectors generated by Warping, First0rder, and Second0rder on image collection original for snapshot positions $(6,4)$ and $(0,16)$. For both snapshot

\footnotetext{
${ }^{1}$ However, the stable filter introduces other boundary effects, see figure 8.11 for an example.
} 
positions FirstOrder exhibits excellent home vector fields. In particular, the field for $(0,16)$ is much improved over Warping. SecondOrder performs moderately well on snapshot position $(6,4)$ where a few incorrect vectors mar the otherwise correct vector field. It performs considerably less well at position $(0,16)$.

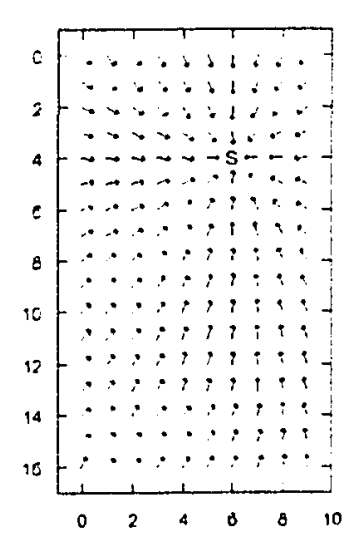

(a) Warping, $\mathrm{s}=(6,4)$ : $A A E=0.119$, $R R=1.000$

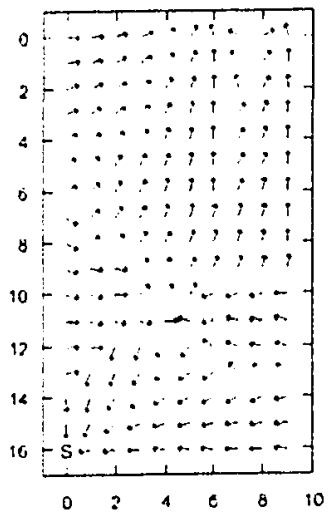

(b) Warping, $\mathbf{s}=(0,16)$ : $A A E=1.758$, $R R=0.266$

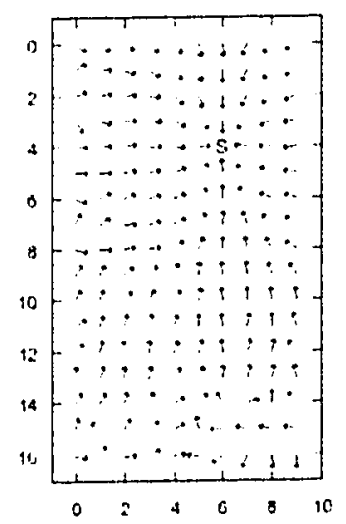

(e) Secondorder.

$$
\begin{aligned}
& \mathrm{s}=(6,-1): \\
& A . A E=0.363, \\
& R R=0.982
\end{aligned}
$$

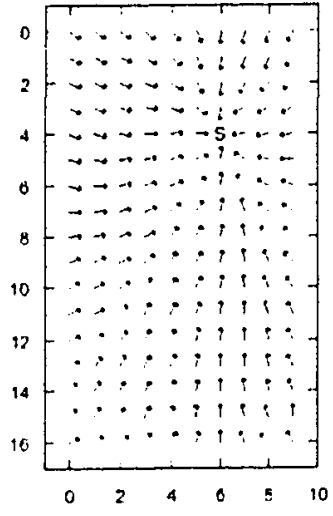

(c) First0rder, $\mathrm{s}=(6,4)$ : $A . A E=0.200$, $R R=1.000$

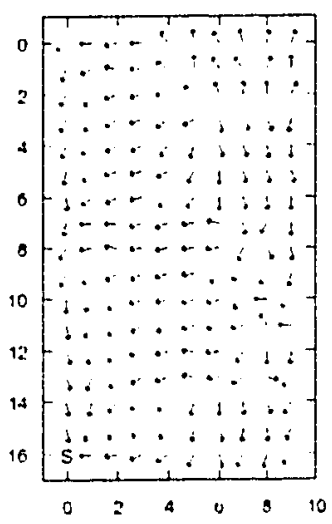

(f) SecondOrder, $\mathrm{s}=(0,16):$ $A . A E=0.911$, $R R=0.710$

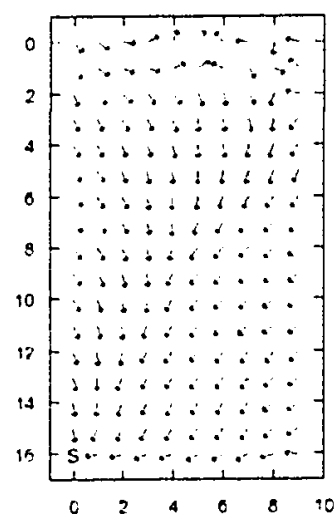

(d) FirstOrder, $\mathbf{s}=(0,16):$ $A A E=0.564$, $R R=0.781$

Figure 9.2: Home vector fields for block matching $(a, b)$ and warping $(c, d)$, for snapshot positions $(6,4)(a, c)$ and $(0,16)(b, d)$ in image collection original.

Figure 9.3 shows the computed $A A E$ and $R R$ for the three methods across snapshot positions. FirstOrder performs quite well across all positions in original. Second0rder 
performs less well, but still exhibits relatively low $A A E$ and high $R R$ across most positions.

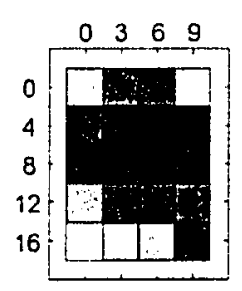

(a) Warping: $A A E_{*}=$ $0.576 \pm 0.461$

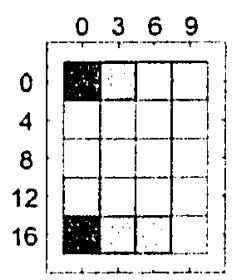

(d) Warping: $R R_{*}=$ $0.792 \pm 0.226$

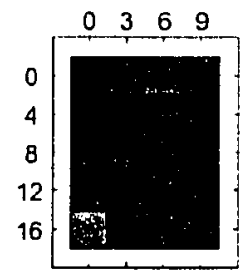

(b) FirstOrder: $A A E_{*}=$ $0.269 \pm 0.106$

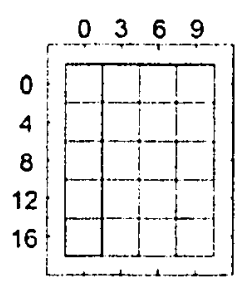

(e) FirstOrder: $R R_{*}=$ $0.975 \pm 0.0504$

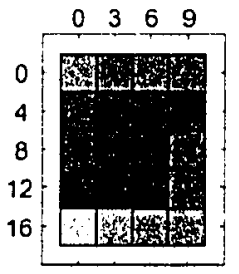

(c) Second0rder: $A A E_{*}=$ $0.492 \pm 0.169$

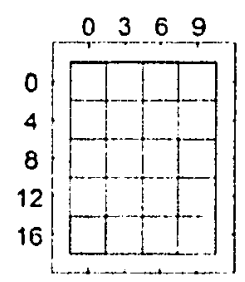

(f) SecondOrder: $R R_{0}=$ $0.887 \pm 0.101$

Figure 9.3: $A A E$ and $R R$ across snapshot positions for all methods on image collection original. Beneath each plot is shown the mean value of the metric $\left(A A E_{*}\right.$ or $\left.R R_{+}\right)$ and its standard deviation (the number following the ' \pm ' symbol). Average values for $A A E \max .=1.758, R R \max .=1$.

\section{Image Collections hall1 and hall2}

Figure 9.4 shows sample home vectors generated by these methods on the hall image collections. On hall1 all three methods generate near perfect home vector fields. On hall2 there is a reduction in performance for Warping, but the performance of both differential methods remains high. The excellent performance of the differential methods is generally maintained across snapshot positions (see figures 9.5 and 9.6).

\section{Cross-Collection Tests}

Figure 9.7 plots $A A E_{*}$ and $R R_{*}$ for the cross-collection tests. The performance of both differential methods are impressive for these tests. Firstorder shows low $A A E$ and particularly high $R R$ for all pairs of image collections except those involving day, doorlit, or 


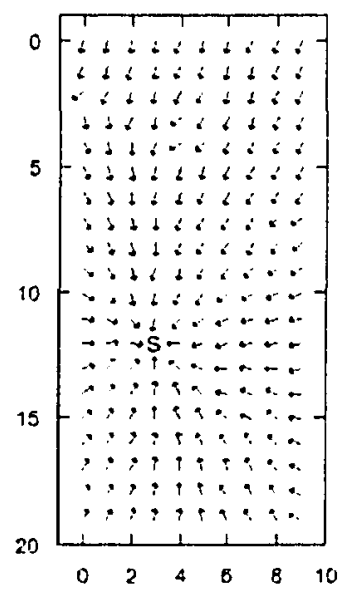

(a) Warping, hall1, $\mathbf{s}=(3,12)$ : $A .4 E=0.160$, $R R=1.000$

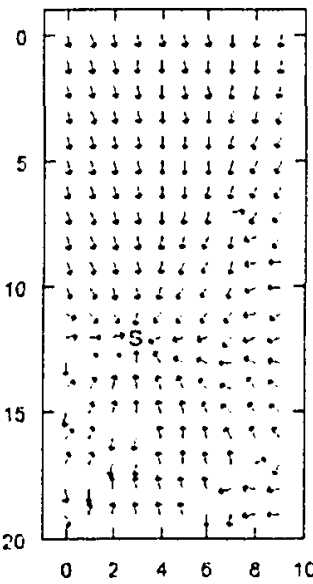

(b) Warping, hall2, $\mathrm{s}=(3,12)$ : $A A E=0.40 \overline{7}$, $R R=0.915$

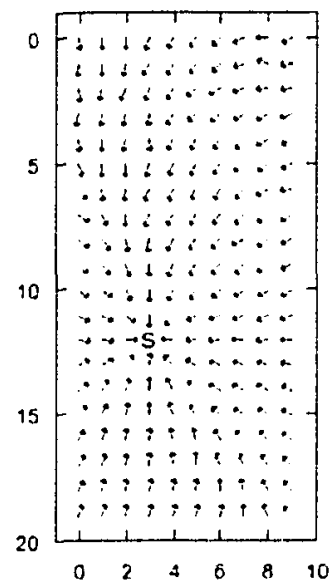

(e) SecondOrder, hall1, $\mathbf{s}=(3,12)$ : $A A E=0.233$, $R R=1.000$

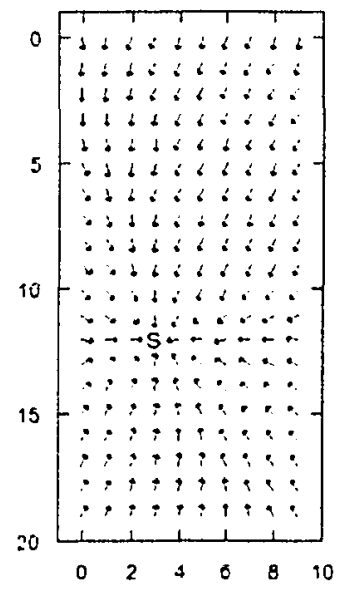

(c) First0rder, hall1. $\mathbf{s}=(3,12):$ $A A E=0.204$, $R R=1.000$

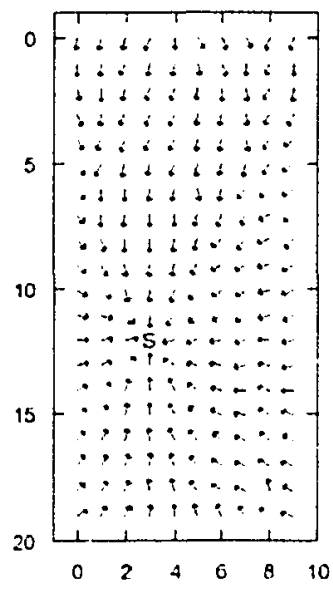

(f) SecondOrder, hall2, $\mathbf{s}=(3,12)$ : $A A E=0.225$, $R R=1.000$

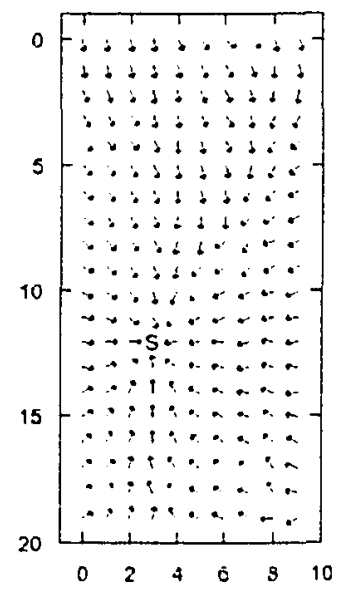

(d) Firstorder, hall2. $\mathrm{s}=(3,12)$ : $A A E=0.30 \mathrm{~s}$. $R R=1.000$

Figure 9.4: Home vector fields for Warping ( $a, b)$ and BlockMatch $(c, d)$, for snapshot. position $(3,12)$ in image collections hall1 (a,c) and hall2 (b,d). 


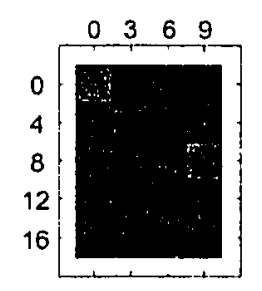

(a) Warping: $A A E_{*}=$ $0.295 \pm 0.151$

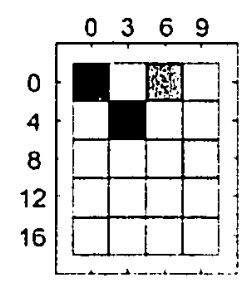

(d) Warping: $R R_{*}=$ $0.841 \pm 0.289$

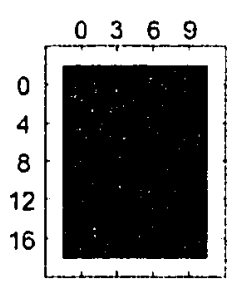

(b) Firstorder: $A A E_{*}=$ $0.312 \pm 0.107$

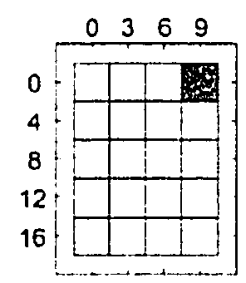

(e) First0rder:

$$
\begin{aligned}
& R R_{+}= \\
& 0.946 \pm 0.144
\end{aligned}
$$

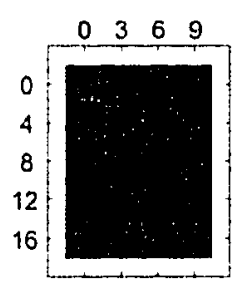

(c) SecondOrder:

$$
\begin{aligned}
& A . A E_{*}= \\
& 0.357 \pm 0.112
\end{aligned}
$$

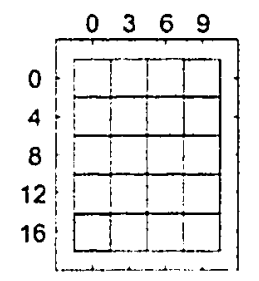

(f) SecondOrder: $R R_{+}=$ $0.951 \pm 0.0865$

Figure 9.5: $A A E$ and $R R$ across snapshot positions for all methods on image collection hall1. $A A E \max .=2.288, R R \max .=1$.

winlit. Secondorder shows remarkably constant performance in both performance metrics for all pairs of image collections, including those involving day, doorlit, and winlit.

\subsection{Comparative Results}

Table 9.1 presents the result of statistical analyses on image collection original, comparing the performance of Warping, Firstorder, and SecondOrder. The table shows that First0rder performs significantly better than either Warping or SecondOrder for both performance metrics.

For image collection hall1, neither the repeated measures ANOVA on $A A E$ nor Friedman's test on $R R$ revealed any significant differences between the performance of Warping, FirstOrder, and SecondOrder (ANOVA $P=0.1335$, Friedman's $P=0.056$ ). Table 9.2 present results of the same tests for image collection hall2. For the $A A E$ metric Second0rder performs significantly better then Warping and there are otherwise no significant differences. For $R R$, both differential methods perform significantly better than 


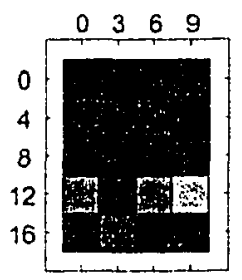

(a) Warping: $A A E_{*}=$ $0.457 \pm 0.185$

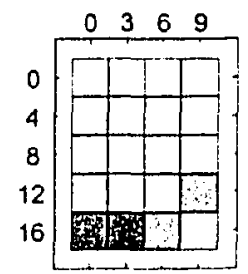

(d) Warping:

$R R_{+}=$ $0.798 \pm 0.202$

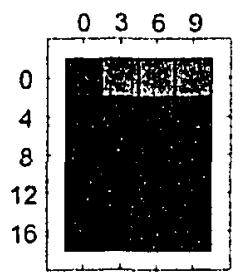

(b) First0rder: $A A E$. $=$ $0.378 \pm 0.168$

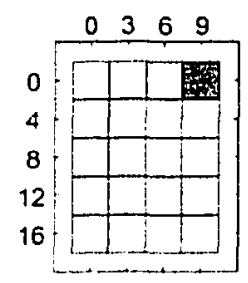

(e) FirstOrder: $R R_{*}=$ $0.935 \pm 0.152$

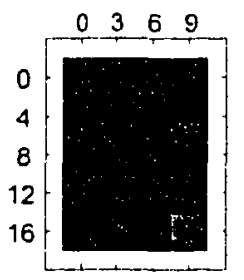

(c) Secondorder: $A A E_{*}=$ $0.314 \pm 0.0962$

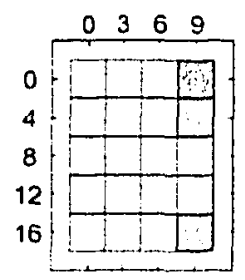

(f) SecondOrder: $R R_{*}=$ $0.926 \pm 0.162$

Figure 9.6: $A A E$ and $R R$ across snapshot positions for all methods on image collection hall2. $A A E$ max. $=2.12 \bar{i}, R R$ max. $=1$.

Warping.

Statistical analysis of the cross-collection tests is reported in table 9.3 . For both performance metrics Firstorder and Second0rder outperform warping.

Both differential methods exhibit extremely impressive performance, despite their simplicity. In no case does Warping exhibit better performance. Indeed, Warping is competitive with the differential methods only in image collection hall1 where there were no significant differences. Otherwise, one or both of the differential methods outperformed warping. The only significant differences found between FirstOrder and SecondOrder were on image collection original where First0rder was found to be superior.

\subsection{Analysis}

The results above indicate that the underlying assumptions of the differential methods must have been satisfied to some extent. In particular, some interesting insight into the operation of these methods is gained by examining how the angular error changes with respect to the 


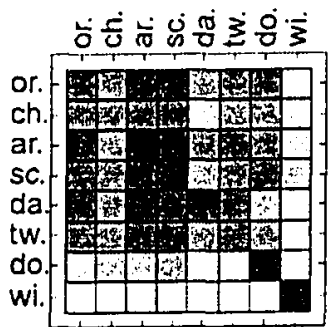

(a) Warping: $A A E_{*}(*, *)=$ $0.836 \pm 0.361$

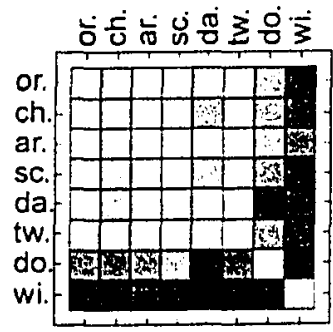

(d) Warping: $R R .(*, *)=$ $0.551 \pm 0.251$

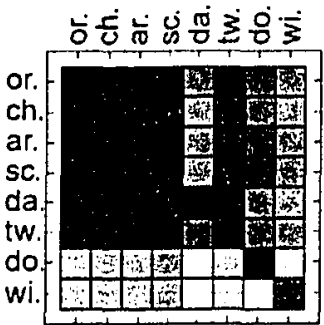

(b) First0rder: $A A E_{*}(*, *)=$ $0.591 \pm 0.317$

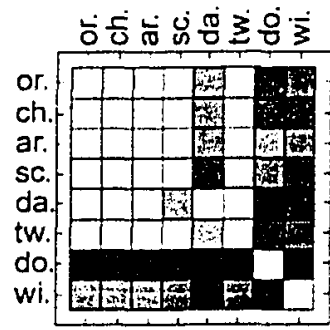

(e) FirstOrder: $R R_{*}(*, *)=$ $0.61+ \pm 0.339$

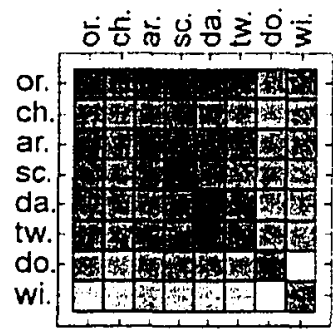

(c) Second0rder: $A A E_{*}(*, *)=$ $0.621 \pm 0.171$

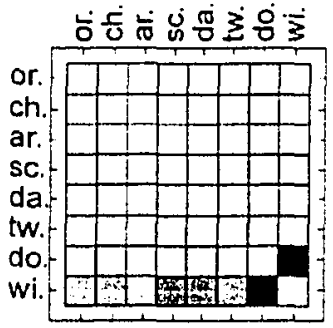

(f) Second0rder: $R R_{*}(*, *)=$ $0.751 \pm 0.156$

Figure 9.7: $A A E_{*}$ and $R R_{*}$ over all pairings of the eight office image collections. See figure 4.9 for notation. $A A E \max$. $=1.811, R R \max .=1$.

Significance of difference in $A . A E_{*}$ (original)

\begin{tabular}{|l|l|l|l|}
\hline & Warping & FirstOrder & SecondOrder \\
\hline Warping & $\mathrm{X}$ & & \\
\hline FirstOrder & $* * *$ & $\mathrm{X}$ & $* *$ \\
\hline SecondOrder & & & $\mathrm{X}$ \\
\hline
\end{tabular}

Significance of difference in $R R_{*}$ (original)

\begin{tabular}{|l|l|l|l|}
\hline & Warping & First0rder & Second0rder \\
\hline Warping & $\mathrm{X}$ & & \\
\hline FirstOrder & $* *$ & $\mathrm{X}$ & $* *$ \\
\hline SecondOrder & & & $\mathrm{X}$ \\
\hline
\end{tabular}

Table 9.1: Statistical significance of the difference in $A A E_{*}$ (original) and $R R_{*}$ (original) between homing methods. See table 4.1 for notation. 
Significance of difference in $A A E_{*}$ (hall2)

\begin{tabular}{|l|l|l|l|}
\hline & Warping & FirstOrder & SecondOrder \\
\hline Warping & $\mathrm{X}$ & & \\
\hline FirstOrder & & $\mathrm{X}$ & \\
\hline SecondOrder & $* *$ & & $\mathrm{X}$ \\
\hline
\end{tabular}

Significance of difference in $R R_{*}$ (hall2)

\begin{tabular}{|l|l|l|l|}
\hline & Warping & FirstOrder & SecondOrder \\
\hline Warping & $\mathrm{X}$ & & \\
\hline FirstOrder & $*$ & $\mathrm{X}$ & \\
\hline SecondOrder & $*$ & & $\mathrm{X}$ \\
\hline
\end{tabular}

Table 9.2: Statistical significance of the difference in $A A E_{*}$ (hal12) and $R R_{*}$ (hal12) between homing methods. See table 4.1 for notation.

Significance of difference in $A A E_{*}(*, *)$

\begin{tabular}{|l|l|l|l|}
\hline & Warping & FirstOrder & SecondOrder \\
\hline Warping & $\mathrm{X}$ & & \\
\hline FirstOrder & $* * *$ & $\mathrm{X}$ & \\
\hline SecondOrder & $* * *$ & & $\mathrm{X}$ \\
\hline
\end{tabular}

Significance of difference in $R R_{+}(*, *)$

\begin{tabular}{|l|l|l|l|}
\hline & Warping & FirstOrder & SecondOrder \\
\hline Warping & $\mathrm{X}$ & & \\
\hline FirstOrder & $* * *$ & $\mathrm{X}$ & \\
\hline SecondOrder & $* * *$ & & $\mathrm{X}$ \\
\hline
\end{tabular}

Table 9.3: Statistical significance of the difference in $A A E_{*}(*, *)$ and $R R_{*}(*, *)$ between homing methods for all pairings of image collections from the office environment. See table 4.1 for notation. 
location of the foci. Recall that the requirement for small feature shifts should be most satisfied near the foci.

From the perspective of analysis, we know the true home vector. We can compare this vector with every computed home vector and find the angle between them. As both differential methods compute home vectors at every pixel, we can compute an error image for every processed image. Figure 9.8 shows error images for both First0rder and SecondOrder at snapshot position $(6,8)$ and current position $(5,8)$ of image collection original. In figure 9.8 (a) the amount of error is not noticeably different near the foci than for other regions of the error image. In figure 9.8(b), the error does appear somewhat lower near the foci.

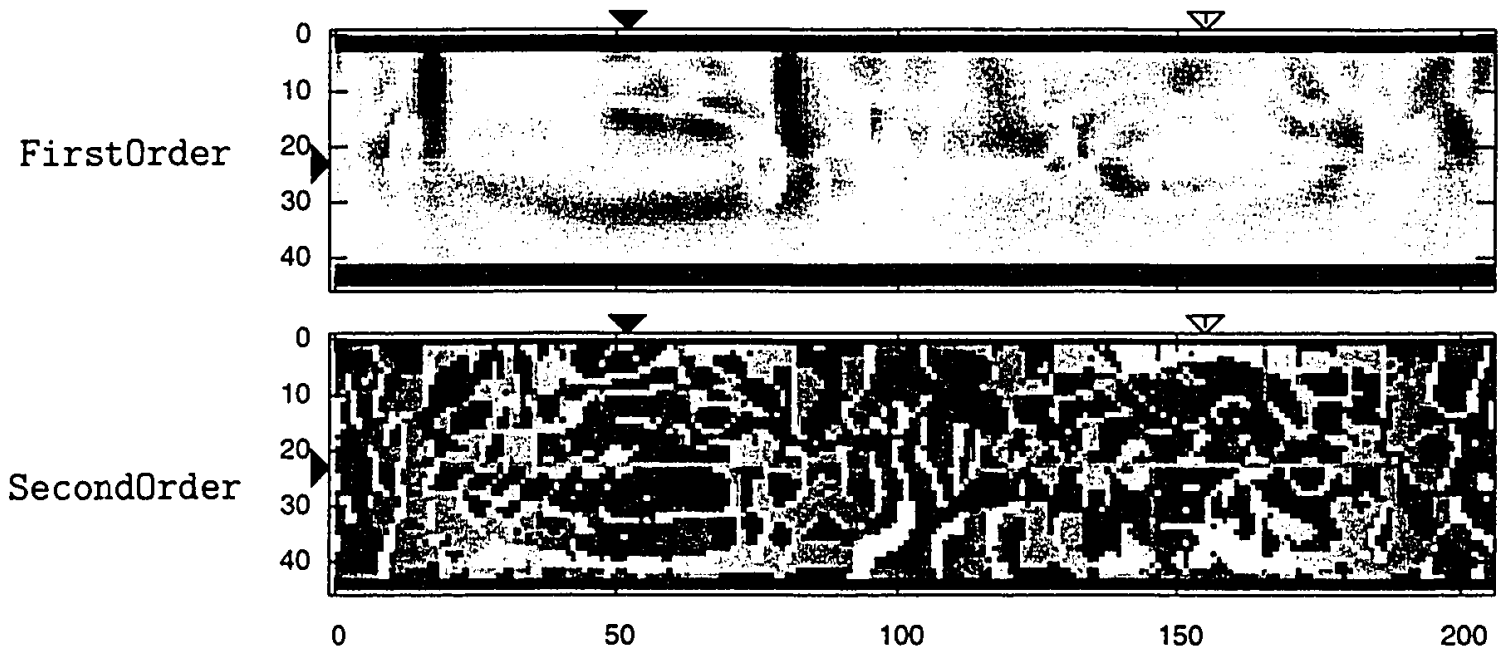

Figure 9.8: Error images for both differential methods at snapshot position $(6,8)$ and current position $(5,8)$. Lighter pixels indicate higher angular error. Ideal positions of the FOC and FOE are indicated as in figure 8.8.

Examining single images such as those in figure 9.8 is inconclusive. A single error image is specific to a particular pair of snapshot and current positions. We can obtain a more robust indication of where the error lies by combining error images from multiple pairs of snapshot and current positions. 3400 such pairs were obtained for image collection original by selecting one of the collection's 170 images as the current image, and an image from one of the 20 positions shown in figure 3.6 as the snapshot image. 3400 error images were generated for both differential methods. As we are interested in where the error lies with respect to the FOC and FOE, each of these error images was rotated such that the ideal location of the FOC was at position 0 , and that of the FOE was at position $w / 2$, where $w$ 
is the width of the image. An average error image was then be obtained by summing all 3400 rotated error image and dividing each pixel by 3400 .

Figure 9.9 shows average error images from both differential methods. Also shown are one-dimensional plots from the same data, obtained by averaging the columns of the average error images. It is clear that error is lower at the locations of the FOC and FOE. This lends credence to the assumption of small shifts at the foci. The fact that we are using a panoramic imaging system is important because it means that both foci are always in view. A narrow field of view could dramatically reduce the effectiveness of the differential methods. Yet a complete $360^{\circ}$ image may not be required. If the imaging system has just a hemispherical $\left(180^{\circ}\right)$ field of view then one of the foci will always be in view. For a larger field of view such as that of the honeybee (ca. $310^{\circ}$ [59]) both foci would be in view for most orientations.

\subsection{Complexity and Biological Plausibility}

The differential methods require no search for correspondences. This search was the primary component in previous measures of computational complexity: For the differential methods, the computational cost per-pixel depends on the kernels applied and the implementation details of the required functions. A rough analysis of First0rder in combination with the approximate vector mapping method reveals that only about 34 low-level operations (e.g. additions, multiplications, $\sqrt{\cdot}, \operatorname{sgn}())$ are required per pixel. At each pixel an estimated home vector is computed. These are finally summed together to produce the final home vector. This summing process can be incorporated into the per-pixel cost. vielding 35 low-level operations for the first-order method. For SecondOrder, about 51 operations are required. On a Pentium 4 2GHz PC Firstorder requires 11.4 ms on average to process an image and compute a home vector. Second0rder requires $33.8 \mathrm{~ms}$.

Both differential methods involve only retinotopic-compatible operations, except for the final summation of home vectors which is a convergent summing operation. These methods easily satisfy the constraints for retinotopic processing.

\subsection{Conclusion}

This chapter proceeded from insights obtained in the previous clapter to test differential methods for visual homing. These methods were found to be surprisingly successful, despite their reliance on assumptions which are often unfulfilled. The convergence of several factors 


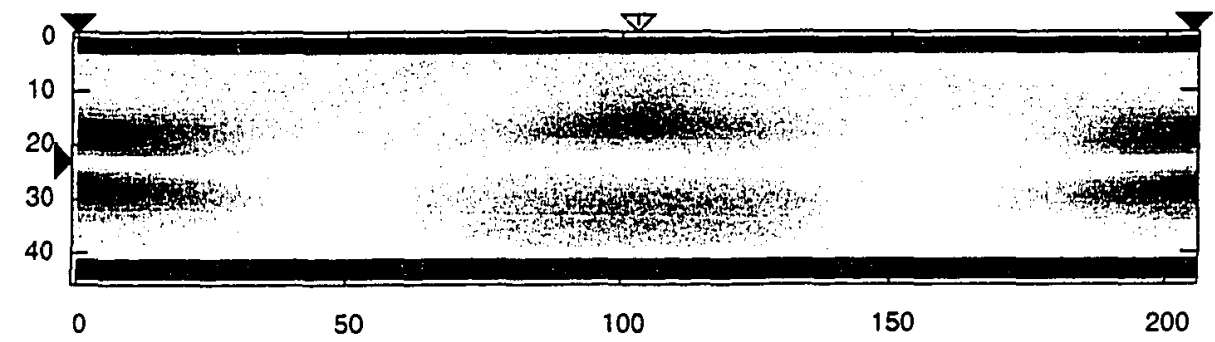

(a) Average error image for Firstorder

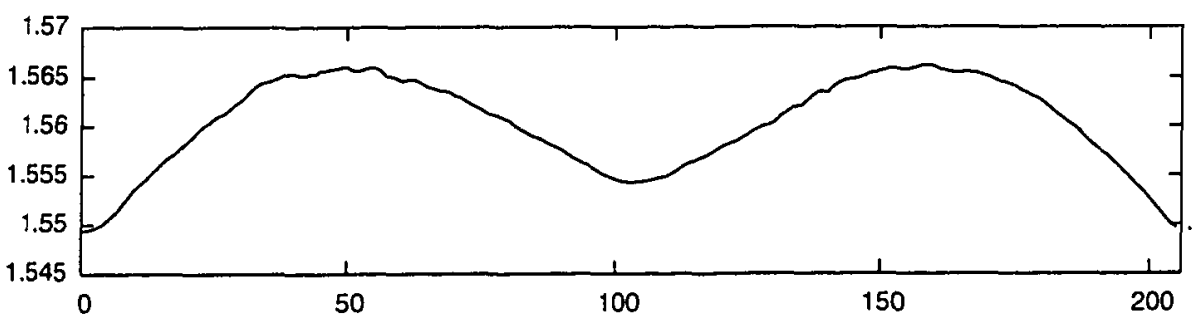

(b) Sum of columns of image in (a)

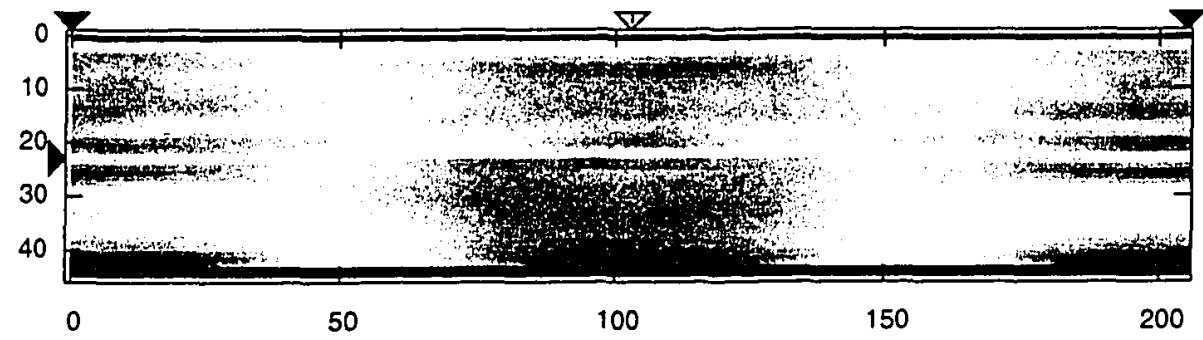

(c) Average error image for Second0rder

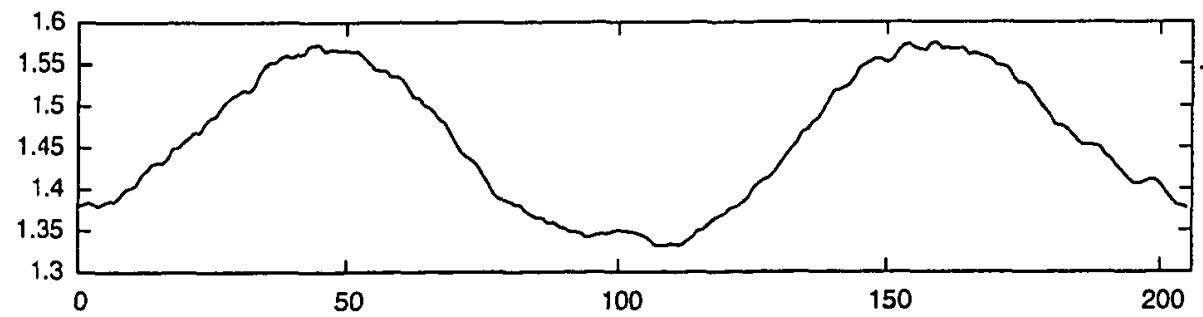

(d) Sum of columns of image in (c)

Figure 9.9: Average error images and one-dimensional plots for both differential methods on image collection original. 
allows the differential methods to succeed. The prevalence of low-frequency image features reduces the error due to the Taylor approximation. This error is further reduced wherever the shift of features between the snapshot and current images is small. The insights of chapter 2 allowed us to suppose that the small shifts at the foci would reduce error, and the analysis above confirmed this supposition. The use of panoramic images guarantees that both foci are always in view. Finally, the uncorrelated mismatches assumption permits successful homing despite deviations from these assumptions.

The finding that differential-based techniques can be used for visual homing comes as quite a surprise. Such techniques have been in use by the computer vision community since at least 1981 [45]. They have been employed in the study of egomotion [27, 94] and in inferring the structure of the environment from the optic fiow field (see [96], chapter 8). However, it is always assumed that accurate flow fields can be estimated by differential flow fields only for small movements. Also, these methods exhibit the aperture problem whereby flow can only be estimated in the direction of the image gradient. Therefore differential techniques were naturally excluded from consideration for visual homing. At the time of this writing, no record of their use in visual homing can be found. The finding that lowfrequency image components were sufficient for homing prompted their investigation here.

The concluding chapter which follows will place the differential methods in context and describe how these methods open up new possibilities for implementing visual homing behaviours in robots, and understanding such behaviours in animals. 


\section{Chapter 10}

\section{Conclusions, Contributions, and Future Research}

\subsection{Introduction}

This final chapter has three major components. The first is a section describing the primary conclusions that have been arrived at through the course of this research. The second component is a detailed account of the new knowledge contributed by this thesis, and by the published work that underpins it. The third and final component outlines a number of promising directions for future research.

\subsection{Conclusions}

\subsubsection{Primary Conclusion}

The chapter describing the differential methods, First0rder and SecondOrder, showed that these methods exhibited performance compctitive with Franz et al.'s Warping method. Further, the differential methods consist only of simple local operations, entirely consistent with the constraints for retinotopic processing. Therefore, the differential methods satisfy the constraints of the research question (repeated from section 1.5):

"Can the visual homing problem be solved by methods which are both competitive in performance, and yet adhere to the constraints of retinotopic processing?". 
The answer to this question is affirmative and the differential methods represent the best known methods which satisfy these criteria. This is the primary conclusion to be drawn from this work.

\subsubsection{Other Major Conclusions}

Two other significant findings about the visual homing problem were made in the course of this research. These findings explain the success of the differential methods, as well as the success of other methods discovered along the path.

\section{Rich image descriptors are unnecessary}

The first is that rich image descriptors are unnecessary for visual homing. This conclusion was not arrived at all at once or even within a single chapter. The notion of using rich image descriptors arose as a possible means to improve upon the performance of CGSM. This approach was taken up in chapter 7 where a new scale invariant image descriptor was proposed and found to be successful. Yet, the descriptor's scale invariant properties were not found to be crucial to the method's success. This prompted an investigation into block matching. The fact that BlockMatch exhibited performance competitive with Warping showed that the minimal image descriptor did not require sophisticated invariance properties. The analysis of BlockMatch then led to the proposal of IntMatch and GradMatch. These methods employed the absolute minimum possible image descriptors (intensity and gradient of intensity), yet were still found to be quite successful. The fact that rich image descriptors are unnecessary for successful homing opened up the possibility of using differential-based techniques to avoid search.

\section{Bad correspondences can be tolerated}

The second major finding is that for visual homing many bad correspondences can be tolerated. Section 5.4.1 posited the assumption of uncorrelated mismatches. This assumption has been called upon throughout the thesis to explain the success of methods which were known to make at least some bad correspondences. 'That it can tolerate bad correspondences makes visual homing very different from other problems in computer vision. According to Barron et al. [2], the problem of inferring the three-dimensional structure of the environment from a flow field requires that relative errors in computing the flow field be less than $10 \%$. In such a domain it is therefore necessary to carefully filter the set of possible correspondences. 
It was previously assumed that filtering the set of possible correspondences was also crucial for visual homing. The elaborate approaches for establishing correspondences proposed by Röfer [89], Weber et al. [104], and Gourichon et al. [29] attest to this assumption. Yet Weber et al. concluded that simpler approaches worked nearly as well. Clearly, the set of possible correspondences can be dranatically reduced by first filtering the set of detected landmarks. This is the approach taken by Lehrer and Bianco [62] who used a method inspired by the learning flights of insects. The success of the methods of this thesis show that neither elaborate correspondence strategies, nor landmark filtration schemes are necessary for visual homing. Instead, it is advantageous to use simple correspondence methods and to allow as many features to be matched as possible.

\subsection{Contributions}

This thesis has contributed in many ways to the existing body of knotwledge on visual homing. Contributions have come in the form of data, homing methods, insights, and methodology. These contributions have impacts on fields such as robot navigation, computer vision, and insect navigation. This section reviews these contributions in the order in which they were presented.

The image database described in chapter 3 is itself a contribution of this work. Images were captured in a careful and consistent manner under a variety of controlled modifications to the environment. At the time of this writing, some similar image databases do exist. However, none of these databases samples the same variety of environments at the same high optical quality as the Bielefeld image database.

In chapter 4, which described other competing methods for visual homing, at least two new discoveries about these other methods were made. First, it was found that Hafner's [33] method was dependent upon a global image property which was unreliable in some environments. This property is the image centre-of-mass. In order for Hafner's method to succeed, the direction of the centre-of-mass should vary in a consistent way as the agent moves about its environment. Horvever, for the two hall image collections the centre-of-mass property proved inconsistent and therefore unreliable for homing. A second contribution of this chapter was the finding that Zeil et al.'s [108] method of homing by gradient descent could perform to a reasonable standard within an indoor office environment. Zeil et al. speculated that their method could be regarded as problematic in indoor enviromments because of the sharp transitions and repetitive structure of such environments. The results presented in chapter 4 indicate that, at least for the indoor environments sampled by the 
Bielefeld image database, the smooth well property persisted. However, the presence of local minima in the difference functions affected the method's performance such that it was not competitive with Warping.

The chapter on vector mapping techniques makes a clear contribution. The exact vector mapping technique presented in chapter 5 is an advance over previous approaches such as that of Hong et al. [43]. By using both image dimensions this technique is able to generate correct home vectors from correct correspondence vectors without making any assumptions about the distribution of landmarks in the environment.

The contribution of chapter 6 was the extension of Möller et al.'s [ 76,77$]$ neural snapshot model from a one-dimensional method applicable only in simulation, to a two-dimensional method applicable to real-world images. The underlying idea of both the neural snapshot model and CGSM is the principle of locally determining correspondence vectors from a potential surface.

Chapter 7 proposed a novel image descriptor which exhibits invariance to scale changes. The contributions of this chapter are two-fold. Firstly; the successful performance of SIMatch prompted an analysis which concluded that simpler image descriptors would suffice. This discovery placed us on the path of increasingly simple image descriptors. Secondly; independent of its application to visual homing, the scale invariant inage descriptor developed here may be interesting to the computer vision community: This scale invariant descriptor has a simpler structure than similar descriptors currently popular in computer vision such as Lowe's [66].

The path of increasingly simple image descriptors was followed to its end in chapter 8. The findings of this chapter affirmed the speculation that rich image descriptors are unnecessary for visual homing. This chapter also lends great support to the major conclusion that bad correspondences can be tolerated.

Chapter 9 led to the primary conclusion and an affirmative answer to the research question. The successful demonstration of differential-based techniques for visual homing will have a profound impact on research in this field. Future visual homing methods having high computational costs will have to justify their complexity in the light of the inexpensive and simple differential methods.

This thesis has contributed also to the methodology of research in visual homing. Most previous approaches to robot visual homing have done little more than test their methods on one or two snapshot positions in a single environment. The testing clescribed in this thesis is much more rigorous. Fragile methods which can only succed in particular parts of particular environments would fail the tests used here. Also the use of statistical tests allows us to state the estimated probability that the observed differences in performance 
between methods was the result of chance. This combination of varied test environments and statistical analysis appears to be unprecedented in robot visual homing research.

Finally, while adherence to the constraints of retinotopic processing provides no guarantee that a method has any connection to any animal's homing abilities, these constraints do serve the purpose of keeping this work broadly relevant to biology. This raises the bar for other approaches to visual homing in yet another way.

\subsection{Future Research}

\subsubsection{Orientation}

The assumption was made for all methods developed in this thesis that the snapshot and current images were captured from the same orientation, or that some form of compass was required to align the views. Robustness of methods to small changes in orientation was tested implicitly, simply by the fact that images of the image database were captured from only approximately the same orientation. A more rigorous test of robustness against orientation changes remains to be done. If a method exhibits low sensitivity to orientation changes then it could tolerate inaccuracies in its compass.

It would also be interesting to adapt the methods developed here to recover orientation through vision. Section 3.3.1 mentions a number of methods which have this capacity: It would be challenging to solve this problem while adhering to retinotopic processing. Methods such as the differential methods and CGSM rely upon the presence of small feature shifts. However, if orientation is unconstrained then all feature shifts may be large. One possibility would be to use the coherence of the flow field as an indicator of correct orientation. If the orientation is correct then there would be two opposing regions of coherence, corresponding to the FOC and the FOE. In this case the agent would simply rotate until coherence was achieved. From the technical standpoint it would be preferable if the angle of orientation could be recovered at once. Röfer [89] and Nelson and Aloimonos [80] describe similar approaches to this problem, however both of these require a search process. The approach suggested by Zeil et al. [108] is to use the difference between images to align them by one-dimensional gradient descent. Alternatively, gradient descent could be avoided if a set of snapshot images from different orientations had been stored. In this case, the snapshot image with the smallest difference to the current image would be selected and used for homing. 


\subsubsection{Filtering}

Lehrer and Bianco [62] employed a landmark filtering system based upon the learning flights of insects. Contrary to their approach, it was found here that careful selection of landmarks was not required to achieve successful homing. However, this does not imply that filtering cannot be helpful in certain environments. In order to asses the benefit of filtering it would likely be necessary to take the methods developed here to an environment where their level of performance was degraded. Alternatively; a focus on some of the most difficult environmental modifications tested here (such as original/winlit) might necessitate some manner of landmark filtering system, perhaps also inspired by the learning flights and walks of insects.

\subsubsection{Neural or Evolutionary Optimization}

Visual homing is not required if an agent is able to maintain an accurate home vector by path integration. In theory, such an agent has the capability to train its visual homing system by using the home vector from path integration as the target output and adjusting parameters until the target output is attained. Thus, a supervised learning algorithm could be employed online to optimize the homing method (see Mitchell [70] for a number of such algorithms). This is the approach used by Hafner to arrive at COMALV [33, 34], however it could just as easily be used to optimize any other homing method. Offline approaches are possible as well.

On a broader timescale, and even if the path integration system is unavailable or unreliable, a homing method could be optimized by an evolutionary algorithm. In this case the same training signal could be used, or else one of the performance metrics such as $A . A E$ or $R R$ could be employed to judge the success of a particular individual.

\subsubsection{Hybrid Approaches}

The positive attributes of many of the homing methods discussed in this thesis can be combined in useful ways. Hybrid approaches are suggested whenever two problem solutions have non-overlapping areas of strength.

In terms of the image collections tested here, the two differential methods have complementary areas of strength. Firstorder performed remarkably well on image collection original. SecondOrder performed less well on original but was more consistent overall. Correspondence vectors from both techniques could be combined through averaging. Barron et al. [2] discuss situations where the estimation of second derivatives is poor. If these 
cases could be detected via simple means then they could be used to inlibit the contribution from SecondOrder. This should have the effect of improving performance.

Another attractive hybrid combination would be a pairing of one or both differential methods with Zeil et al.'s [108] method. The differential method(s) would provide the main source of control with a variant of GradDescent acting as a backup. This backup system would compute the SSD between the current and snapshot images, but would otherwise remain inactive as long as the SSD was found to be decreasing. However, if a large increase in SSD was noticed then it would be assumed that the differential methods were failing, or perhaps even malfunctioning. In this case, a version of GradDescent could take over. This combination is suggested not because the results presented here suggest that these approaches are complementary, but because they are both so simple and could easily coexist.

\subsubsection{Relaxing Motion Constraints}

All existing visual homing methods reviewed in chapter 1 , as well as all those presented in this thesis, assume that the agent is confined to a single plane of travel. This is somewhat striking when one considers the influence of the snapshot model [6] which was based on the homing abilities of honeybees. A honeybee, being a flying insect naturally travels in threeclimensional space. However, we assume that a honeybee covers more distance horizontally than vertically. Thus, modeling the honeybee as if it moved within a plane covers much of the effective range of its behaviour. Nevertheless, a visual homing method that could account for changes in height would be more biologically plausible than one which could not $^{1}$. If the assumption that some features remain in common between the snapshot and current images holds true, then an extension to three-dimensional homing might not be that difficult. In this case the main challenge would be in the vector mapping stage. Both the exact vector mapping technique of chapter 5 and the approximate technique of chapter 9 assume travel within the plane. Other techniques would be required for three-dimensional homing (e.g. such as that of Basri et al. [3]).

\subsubsection{Biology}

Of what use to biologists are the methods and insights provided in this thesis? It was argued in chapter 1 that methods which both perform well and adhere to the constraints

\footnotetext{
${ }^{1}$ Such a method might also account for roll, pitch, and yaw (i.e. orientation). However, it is possible that these rotational parameters are controlled by non-visual systems such as the vestibular system for roll and pitch correction (i.e. levelling) and compass systems for yaw.
} 
of retinotopic processing are more biologically plausible. Thus, by considering only these methods, the space of possible explanations for insect visual homing has been reduced. Yet, more specific suggestions can be made.

For IntMatch, GradMatch, and the differential methods it was required that low-frequency image components exist in the image. That low-frequency components exist in natural images is known [90, 97]. It would perhaps be interesting to assess the ability of insects to navigate in a world where low-frequencies were artificially reduced. For example, consider a room where all surfaces are covered with patterns. These patterns could be taken from the set of those known to be recognizable by insects, and scaled so as to be resolvable throughout the room. The patterns would make it possible, in theory, to navigate by highfrequency pattern correspondence. No large-scale features would exist in this room. Thus, homing methods relying on low-frequency components should fail. This could be verified by testing the methods mentioned above on images taken from within this specially prepared environment. It would be interesting to see if insects could navigate in such a space, and if not, what modifications would be required to make it navigable.

Another possible experiment could test the necessity of a wide-field of view for insect navigation. For the differential methods the angular error for homing was lower for image regions near the FOC and FOE. A wide field of view ensures that one or both foci are always visible. Experimental paradigms to test this might include eye caps (Wehner et al. [106] cites two uses of eye caps on Cataglphyis) or varying the angular position of landmarks with respect to the direction of the true home vector [61].

The general point is that different visual homing methods make different assumptions about the world. It may be possible to create environments in which the validity of these assumptions can be controlled. In this way it can at least be ascertained whether a homing insect and a homing algorithm rely on the same assumptions.

\subsubsection{Long-Range Homing}

In chapter 1 visual homing was defined as the ability to home to a goal from a nearby location. The constraint that the start position for homing be 'nearby' can be justified by positing some additional mechanism such as path integration to return the agent from a distant location. Additionally, the scene at the remote location might prompt the recall of a stored home vector, as appears to be the case for honeybees $[8,68]$. It would be very interesting to attempt a robotic implementation of a more complete picture of insect navigation which included visual homing, path integration, recognition-based recall of stored vectors, and also the use of landmarks en route [8]. Such an implementation would focus on 
learning and following an extended route through a cluttered environment. Success of this project would lend credence to the hypothesis that navigation can be achieved by a set of semi-independent yet associated mechanisms. Franz et al. [24, 25] have also made attempts to embed visual homing within a larger navigational context. However, their approach is more concerned with developing and maintaining an internal topological map [23].

\subsection{Summary}

This thesis has investigated the visual homing problem from the perspective that methods relevant to biology can also be among the most effective methods in robotics. The technical success of the methods developed here validates this perspective. It remains to be seen whether insects might employ similar techniques. In any case, it is hoped that the insights developed here will be useful in the ongoing study of these fascinating creatures. 


\section{Appendix A}

\section{Scale Invariant Image Descriptors}

The goal of this appendix is to determine the relationship of $g^{\mathbf{p}, \mathbf{v}, I^{\prime}}$ to $g^{\mathbf{p}, \mathbf{v}, I}$. We begin with $g^{\mathbf{p}, \mathbf{v}, I^{\prime}}$

$$
g^{\mathbf{p}, \mathbf{v}, I^{\prime}}=\int_{0}^{l_{\max }} w(l) I^{\prime}(\mathbf{p}+l \mathbf{v}) d l
$$

From equations ( 7.1$),(7.2)$, and ( 7.4$)$ we obtain

$$
I(\mathbf{p}+l \mathbf{v})=I^{\prime}(\mathbf{p}+k l \mathbf{v})
$$

With a change of variables we have the following:

$$
I\left(\mathbf{p}+\frac{l}{k} \mathbf{v}\right)=I^{\prime}(\mathbf{p}+l \mathbf{v})
$$

We insert the above into the right hand side of equation (A.1) to obtain

$$
g^{\mathbf{p}, \mathbf{v}, I^{\prime}}=\int_{0}^{l_{\max }} w(l) I\left(\mathbf{p}+\frac{l}{k} \mathbf{v}\right) d l
$$

Next we replace the integration variable $l$ with $j=\frac{l}{k}$

$$
g^{\mathbf{p}, \mathbf{v}, I^{\prime}}=\int_{0}^{l_{\max } / k} w(j k) I(\mathbf{p}+j \mathbf{v}) k d j
$$

Now we place our first assumption on $w()$. We assume this function has the property

$$
w(x y)=w(x) w(y)
$$

Utilizing this property on expression (A.5) and renaming the integration variable $j$ back to $l$ gives

$$
g^{\mathrm{p}, \mathrm{v}, I^{\prime}}=k w(k) \int_{0}^{l_{\max } / k} w(l) I(\mathrm{p}+l \mathbf{v}) d l
$$


To proceed further we must place another constraint on $w()$. The intention of this decay function is to reduce the impact of outlying features on $g$. Therefore it makes sense that $w(l)$ should be small for large values of $l$. We first define a new constant $l_{\max }^{*}=\min \left(l_{\max }, l_{\max } / k\right)$. The second constraint on $w()$ is as follows:

$$
w(l) \approx 0 \text { for } l>l_{\max }^{*}
$$

Therefore

$$
g^{\mathbf{p}, \mathbf{v}, I} \approx \int_{0}^{l_{\mathbf{m a x}}} w(l) I(\mathbf{p}+l \mathbf{v}) d l
$$

and

$$
g^{\mathbf{p}, \mathbf{v}, I^{\prime}} \approx k w(k) \int_{0}^{l_{\text {inax }}} w(l) I(\mathbf{p}+l \mathbf{v}) d l
$$

Combining these two approximations gives us the desired relationship

$$
g^{\mathbf{p}, \mathbf{v}, I^{\prime}} \approx k w(k) g^{\mathbf{p}, \mathbf{v}, I}
$$




\section{Bibliography}

[1] A.M. Anderson. A model for landmark learning in the honey-bee. Journal of Comparative Physiology A, 114:335-355, 1977.

[2] J.L. Barron, D.J. Fleet, and S.S. Beauchemin. Performance of optical flow techniques. International Journal of Computer Vision, 12(1):43-77, 1994.

[3] R. Basri, E. Rivlin, and I Shimshoni. Visual homing: Surfing on the epipoles. Technical Report MCS99-10, Weizmann Institute of Science, Mathematics \& Computer Science, 1999.

[4] J. Borenstein, H.R. Everett, and L. Feng. Where am I? Sensors and methods for mobile robot positioning. University of Michigan, 1996.

[5] R.A. Brooks. Intelligence without representation. Artificial Intelligence, 47:139-159, 1991.

[6] B.A. Cartwright and T.S. Collett. Landmark learning in bees. Joumal of Comparative Physiology A, 151:521-543, 1983.

[7] B.A. Cartwright and T.S. Collett. Landmark maps for honeybees. Biological Cybcrnetics, 57:85-93, 1987.

[8] T.S. Collett. Insect navigation en route to the goal: Multiple strategies for the use of landmarks. Journal of Experimental Biology, 199:227-235, 1996.

[9] T.S. Collett and J. Baron. Biological compasses and the coordinate frame of landmark memories in honeybees. Naturc, 368:137-140, 1994.

[10] T.S. Collett and M. Collett. Memory use in insect visual navigation. Nature Reviews Neuroscience, 3:542-552, 2002. 
[11] T.S. Collett and M.F. Land. Visual spatial memory in a hoverfly. Journal of Comparative Physiology, 100:59-84, 1975.

[12] T.S. Collett and J. Zeil. Places and landmarks: an arthropod perspective. In S. Healy, editor, Spatial Representation in Animals, chapter 2. Oxford University Press, 1998.

[13] Fly-By-Day Consulting. Sunrise/sunset computer, 2004. URL http://www. mindspring.com/ cavu/sunset.html.

[14] M. de Berg, M. van Kireveld, M. Overmars, and O. Schwarzkopf. Computational Geometry: Algorithms and Applications. Springer, 1998.

[15] J.L. Devore. Probability and Statistics for Engineering and the Sciences. Brooks/Cole, 1991.

[16] M. Dill, R. Wolf, and M. Heisenberg. Visual pattern recognition in drosphilia involves retinotopic matching. Nature, 365:751-753, 1993.

[17] M. Egelhaaf and A. Borst. Motion computation and visual orientation in flies. Comparative Biochemical Physiology, 104A(4):659-673, 1993.

[18] M. Egelhaaf, R. Kern, H.G. Krapp, J. Kretzberg, R. Kurtz, and A-K. Warzecha. Neural encoding of behaviourally relevant visual-motion information in the fly. Trends in Neurosciences, 25(2):96-102, 2002.

[19] B. Elmmer and W. Gronenberg. Mushroom body volumes and visual interneurons in ants: Comparison between sexes and castes. Joumal of Comparative Neurology. 469: $198-213,2004$.

[20] M. A. Fischler and R. C. Bolles. Random sample consensus: A paradigm for model fitting with applications to image analysis and automated cartography. Communications of the ACM, 24:381-395, 1981.

[21] N. Franceschini, J.M. Pichon, and C. Blanes. From insect vision to robot vision. Philosophical Transactions of the Royal Society of London B, 337:283-294, 1992.

[22] M.O. Franz and H.G. Krapp. Wide-field, motion-sensitive neurons and matched filters for optic flow fields. Biological Cybernetics, 83:185-197, 2000.

[23] M.O. Franz and H.A. Mallot. Biomimetic robot navigation. Robotics and Autonomous Systems, Special Issue: Biomimetic Robots, 30:133-153, 2000. 
[24] M.O. Franz, B. Schölkopf, P. Georg, H.A. Mallot, and H.H. Bülthoff. Learning view graphs for robot navigation. In W. Lewis Johnson and B. Hayes-Roth, editors, Proceedings of the First International Conference on Autonomous Agents (Agents'97), pages 138-147. ACM Press, 1997.

[25] M.O. Franz, B. Schölkopf, H.A. Mallot, and H.H. Bülthoff. Learning view graphs for robot navigation. Autonomous Robots, 5:111-125, 1998.

[26] M.O. Franz, B. Schölkopf, H.A. Mallot, and H.H. Bülthoff. Where did I take that snapshot? Scene-based homing by image matching. Biological Cybernetics, 79:191202, 1998.

[27] J. Gluckman and S. Nayar. Ego-motion and omnidirectional cameras. In Proceedings of the International Conference on Computer Vision, pages 999-1005, 1998.

[28] S. Gourichon, J.-A. Meyer, S. Ieng, L. Smadja, and R. Benosman. Estimating egomotion using a panoramic sensor: Comparison between a bio-inspired and a cameracalibrated method. In H. Holstein and F. Labrosse, editors, AISB03 Symposium on Biologically Inspired Vision, Theory and Application, pages 91-101, 2003.

[29] S. Gourichon, J.-A. Meyer, and P. Pirim. Using colored snapshots for short-range guidance in mobile robots. International Journal of Robotics and Automation, Special Issue: Biologically Inspired Robotics, 17(4):154-162, 2002.

[30] P. Graham, , V. Durier, and T.S. Collett. The binding and recall of snapshot memories in wood ants (Formica rufa L.). Journal of Experimental Biology, 207:393-39S, 2003.

[31] P. Graham and T.S. Collett. View-based navigation in insects: how wood ants (Formica rufa L.) look at and are guided by extended landmarks. Joumal of Experimental Biology, 205:2499-2509, 2002.

[32] B. Greiner, W.A. Ribi, and W.T. Wcislo. Neural organisation in the first optic ganglion of the nocturnal bee Megalopta genalis. Cell Tissue Research, 318:429-437, 2004.

[33] V.V. Hafner. Adaptive homing - robotic exploration tours. Adaptive Behavior, 9(4): 131-141, 2001.

[34] V.V. Hafner. Adaptive Navigation Strategics in Biorobotics: Visual Homing and Cognitive Mapping in Animals and Machines. PhD thesis, University of Zurich, 2004. 
[35] V.V. Hafner and R. Möller. Learning of visual navigation strategies. In M. Quoy, P. Gaussier, and J. Wyatt, editors, Proceedings of the European Workshop on Learning Robots (EWLR-9), pages 47-56, 2001.

[36] M.T. Hagan, H.B. Demuth, and M. Beale. Neural Network Design. PWS, 1996.

[37] C. Harris and M. Stephens. A combined corner and edge detector. In Proceedings of the Fourth Alvey Vision Conference, pages 147-151, 1988.

[38] R.R. Harrison. Fly-inspired VLSI vision sensors. In J.L. Davis J. Ayers and A. Rudolph, editors, Neurotechnology for Biomimetic Robots, chapter 2. MIIT Press, 2002.

[39] R.I. Hartley and A. Zisserman. Multiple View Geometry in Computer Vision. Cambridge University Press, 2004.

[40] M.H. Hassoun and A.J. Sanghvi. Fast computation of optimal paths in two- and higher-dimensional maps. Neural Networks, 3:355-363, 1990.

[41] S. Healy: Spatial Representation in Animals. Oxford University Press, 1998.

[42] H. Hertel. Processing of visual information in the honeybee brain. In R. Menzel and A. Mercer, editors, Neurobiology and Behavior of Honeybees, pages 141-15i. Springer Verlag, 1987.

[43] J. Hong, X. Tan, B. Pinette, R. Weiss, and E.M. Riseman. Image-based homing. In Proceedings of the 1991 IEEE International Conference on Robotics and Automation, Sacremento, CA, pages 620-625, 1991.

[44] J. Hong, X. Tan, B. Pinette, R. Weiss, and E.M. Riseman. Image-based homing. IEEE Control Systems, pages 38-45, February 1992.

[45] B.K.P. Horn and B.G. Schunck. Determining optical flow. Technical Report 572, Artificial Intelligence Laboratory, MIT, 1980.

[46] A. Horridge. Pattern and 3d vision of insects. In Visual Navigation, pages 26-59. Erlbaum, 1997.

[47] D.H. Hubel and T.N. Wiesel. Receptive fields, binocular interaction and functional architecture in the cat's visual cortex. Journal of Physiology, 160:106-154, 1962. 
[48] S.A. Huber, M.O. Franz, and H.H. Bültoff. On robots and flies: Modeling the visual orientation behavior of flies. Robotics and Autonomous Systems, 29:227-242, 1998.

[49] J.R. Jain and A.K. Jain. Displacement measurement and its application in interframe image coding. IEEE Transactions on Communications, 29(12):1799-1808, 1981.

[50] D.H. Janzen. Euglossine bees as long-distance pollinators of tropical plants. Science, 171:203-205, 1971.

[51] M. Jogan and A. Leonardis. Robust localization using panoramic view-based recognition. In International Conference on Pattern Recognition, pages 136-139, 2000.

[52] S.P.D. Judd and T.S. Collett. Multiple stored views and landmark guidance in ants. Nature, 392:710-714, 1998.

[53] W. Junger. Waterstriders compensate for drift with a discontinuously working visual position servo. Journal of Comparative Physiology, 169(5):633-639, 1991.

[54] H.G. Krapp. Neuronal matched filters for optic flow processing in flying insects. International Review of Neurobiology, 44:93-120, 2000.

[55] H.G. Krapp and R. Hengstenberg. Estimation of self-motion by optic flow processing in single visual interneurons. Nature, 384:463-466, 1996.

[56] B.J. Kuipers and Y.-T Byun. A robot exploration and mapping strategy based on a semantic hierarchy of spatial representations. Journal of Robotics and Autonomous Systems, 8:47-63, 1991.

[57] D. Lambrinos. Navigation in biorobotic agents. PhD thesis, Department of Computer Science, University of Zurich, Switzerland, 1999.

[58] D. Lambrinos, R. Möller, T. Labhart, R. Pfeifer, and R. Wehner. A mobile robot employing insect strategies for navigation. Robotics and Autonomous Systems, Special Jssue: Biomimetic Robots, 30:39-64, 2000.

[59] M.F. Land. Variations in the structure and design of compound eyes. In Stavenga and Hardie, editors, Facets of Vision, chapter 5. Springer-Verlag, 1989.

[60] M.F. Land. Visual acuity in insects. Annual Review of Entomology, 42:147-177, 1997. 
[61] M. Lehrer. How bees use peripheral eye regions to localize a frontally positioned target. Journal of Comparative Physiology A, 167:173-185, 1990.

[62] M. Lehrer and G. Bianco. The turn-back-and-look behaviour: bee versus robot. Biological Cybernetics, 83:211-229, 2000.

[63] J.J. Leonard and H.F. Durrant-Whyte. Simultaneous map building and localization for an autonomous mobile robot. In IROS-91 (Osaka, Japan, 1991), pages 1442-1447, 1991.

[64] T. Lindeberg. Scale-space: A framework for handling image structures at multiple scales. In Procecdings of the CERN School of Computing, Egmond aan Zee, 1996.

[65] D.G. Lowe. Object recognition from local scale-invariant features. In International Conference on Computer Vision, pages 1150-1157, 1999.

[66] D.G. Lowe. Distinctive image features from scale-invariant keypoints. International Journal of Computer Vision, 60(2):91-110, 2004.

[67] A. Mellinger. Creating a milky way panorama on the computer, 2001. URL http: //canopus.physik. uni-potsdam.de/ axm/articles.html.

[68] R. Menzel, K. Geiger, J. Joerges, U. Müller, and L. Chittlia. Bees travel novel homeward routes by integrating separately acquired vector memories. Animal Behavior, $55: 139-152,1998$.

[69] K. Mikolajczyk and C. Schmid. A performance evaluation of local descriptors. In International Conference on Computer Vision $\mathcal{B}$ Pattern Recognition, volume 2, pages 257-263, June 2003.

[70] T. M. Mitchell. Machine Learning. McGraw-Hill, 1997.

[71] R. Möller. Path planning using hardware time delays. IEEE Transactions on Robotics and Automation, 15(3):588-591, 1999.

[72] R. Möller. Insect visual homing strategies in a robot with analog processing. Biological Cybernetics, 83(3):231-243, 2000.

[73] R. Möller. Do insects use templates or parameters for landmark navigation. Journal of Theoretical Biology, 210(1):33-45, 2001. 
[74] R. Möller. A biorobotics approach to the study of insect visual homing strategies, Habilitationssclurift, Universität Zürich, 2002.

[75] R. Möller, D. Lambrinos, T. Roggendorf, R. Pfeifer, and R. Wehner. Insect strategies of visual homing in mobile robots. In B. Webb and T. Consi, editors, Biorobotics Methods and Applications, pages 37-66. AAAI Press / MIT Press, 2001.

[76] R. Möller, M. Maris, and D. Lambrinos. A neural model of landmark navigation in insects. Neurocomputing, 26-27:801-808, 1999.

[77] R. Möller, M. Maris, and D. Lambrinos. A neural model of landmark navigation in insects. Technical Report IFI-AI-99.19, Artificial Intelligence Lab, University of Zurich, 1999.

[78] R.G.M. Morris. Spatial localization does not require the presence of local cues. Learning and Motivation, 12:239-260, 1981.

[79] R. Nelson. Visual homing using associative memory. In Proc. of the Image Understanding Workshop, pages 245-262, Palo Alto, CA, 1989. Morgan Kaufmann.

[80] R. Nelson and A. Aloimonos. Finding motion parameters from spherical motion fields (or the advantage of having eyes in the back of your head). Biological Cybernetics, $58: 261-273,1988$.

[81] R.C. Nelson. Visual homing using an associative memory: Biological Cybernetics, 65: 281-291, 1991.

[82] D.J. Nicholson, S.P.D. Judd, B.A. Cartwright, and T.S. Collett. Learning walks and landmark guidance in wood ants (Formica rufa). Journal of Expcrimental Biology, 202:1831-1838, 1999.

[83] A.V. Oppenheim, A.S. Willsky, and I.T. Young. Signals and Systems. Prentice Hall, 1983.

[84] J.P.J. Pinel. Biopsychology, chapter 7. Allyn and Bacon, 1997.

[85] S.C. Pratt, S.E. Brooks, and N.R. Franks. The use of edges in visual navigation by the ant Leptothorax albipemnis. Ethology, 107:1125-1136, 2001. 
[86] W.A. Ribi. The structural basis of information processing in the visual system of the bee. In R. Menzel and A. Mercer, editors, Neurobiology and Behavior of Honeybees, pages 130-140. Springer Verlag, 1987.

[87] F.C. Rind. Collision avoidance: from the locust eye to a seeing machine. In M.V. Srinivasan and S. Venkatesh, editors, From living eyes to seeing machines, chapter 6. Oxford University Press, 1997.

[88] A. Rizzi, D. Duina, S. Inelli, and R. Cassinis. A novel visual landmark matching for a biologically inspired homing. Pattern Recognition Letters, 22:1371-1378, 2001.

[89] T. Röfer. Controlling a wheelchair with image-based homing. In Proceedings of AISB Workshop on Spatial Reasoning in Mobile Robots and Animals, Manchester, UK, 1997.

[90] D.L. Ruderman and W. Bialek. Statistics of natural images: Scaling in the woods. Physical Review Letters, 73(6):814-817, 1994.

[91] R. Schwind. Size and distance perception in compound eyes. In Stavenga and Hardie, editors, Facets of Vision, chapter 19. Springer-Verlag, 1989.

[92] M.V. Srinivasan, S.W. Zhang, M. Lehrer, and T.S. Collett. Honeybee navigation en route to the goal: Visual flight control and odometry: Joumal of Experimental Biology, 199:237-244, 1996.

[93] J. Stewart. Calculus: Early Transcendentals. Brooks/Cole, second edition, 1991.

[94] I. Stratmann. Onnidirectional imaging and optical flow. In Proceedings of the Third Workshop on Omnidirectional Vïsion, pages 104-114, 2002.

[95] W. Stürzl and H.A. Mallot. Vision-based homing with a panoramic stereo sensor. In Biologically Motivated Computer Vision, Lecture Notes in Computer Science 2525, pages 620-628. Springer, 2002.

[96] E. Trucco and A. Verri. Introductory Techniques for 3-D Computer Vision. Prentice Hall, 1998.

[97] A. van der Schaaf and J.H. van Hateren. Modelling the power spectra of natural images: Statistics and information. Vision Research, 36(17):2759-2770, 1996. 
[98] A. Vardy and R. Möller. Biologically plausible visual homing methods based on optical flow techniques. Connection Science, Special Issue: Navigation, 2005 (to appear).

[99] A. Vardy and F. Oppacher. Low-level visual homing. In W. Banzhaf, T. Christaller, P. Dittrich, J. T. Kim, and J. Ziegler, editors, Advances in Artificial Life - Proceedings of the 7th European Conference on Artificial Life (ECAL), volume 2801 of Lecture Notes in Artificial Intelligence, pages 875-884. Springer, 2003.

[100] A. Vardy and F. Oppacher. Anatomy and physiology of an artificial vision matrix. In A.J. Ijspreet, M. Murata, and N. Wakamiya, editors, Biologically Inspired Approaches to Advanced Information Technology: First International Workshop, BioADIT 2004, volume 3141 of Lecture Notes in Computer Science, pages 290-305. Springer, 2004.

[101] A. Vardy and F. Oppacher. A scale invariant neural feature detector for visual homing. In G. Palm and S. Wermter, editors, Proceedings of the Workshop on Neurobotics, German Conference on Artificial Intelligence, Ulm, 2004.

[102] A. Vardy and F. Oppacher. A scale invariant local image descriptor for visual homing. In G. Palm and S. Wermter, editors, Biomimetic neural leaming for intelligent robots. Springer (to appear), 2005.

[103] B. Webb. What does robotics offer animal behaviour. Animal Behaviour, 60:545-558, 2000.

[104] K. Weber, S. Venkatesh, and M. Srinivasan. Insect-inspired robotic homing. Adaptive Behavior, $\mathrm{\tau}: 65-97,1999$.

[105] R. Wehner. Spatial vision in arthropods. In H. Autrum, editor, Handbook of Sensory Physiology: Vision in Invertcbrates, volume VII/6 C, chapter 4, pages 287-617. Springer, 1981.

[106] R. Wehner, B. Michel, and P. Antonsen. Visual navigation in insects: Coupling of egocentric and geocentric information. Jourmal of Expcrimental Biology, 199:129-140, 1996.

[107] J.H. Zar. Biostatistical Analysis. Prentice Hall, 1996.

[108] J. Zeil, M. Hofmann, and J. Chahl. Catchment areas of panoramic snapshots in outdoor scenes. Journal of the Optical Society of America A, 20(3):450-469, 2003. 
[109] J. Zeil, A. Kelber, and R. Voss. Structure and function of learning flights in bees and wasps. Joumal of Experimental Biology, 199:245-252, 1996. 